US Army Corps of Engineers ${ }_{\circledast}$

Engineer Research and

Development Center

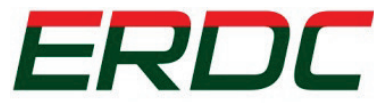

INNOVATIVE SOLUTIONS

for a safer, better world

Innovations for Navigation Projects Research Program

\title{
Corroded Anchor Structure Stability/ Reliability (CAS_Stab-R) Software for Hydraulic Structures
}

Terry W. Warren, Barry C. White, and Robert M. Ebeling

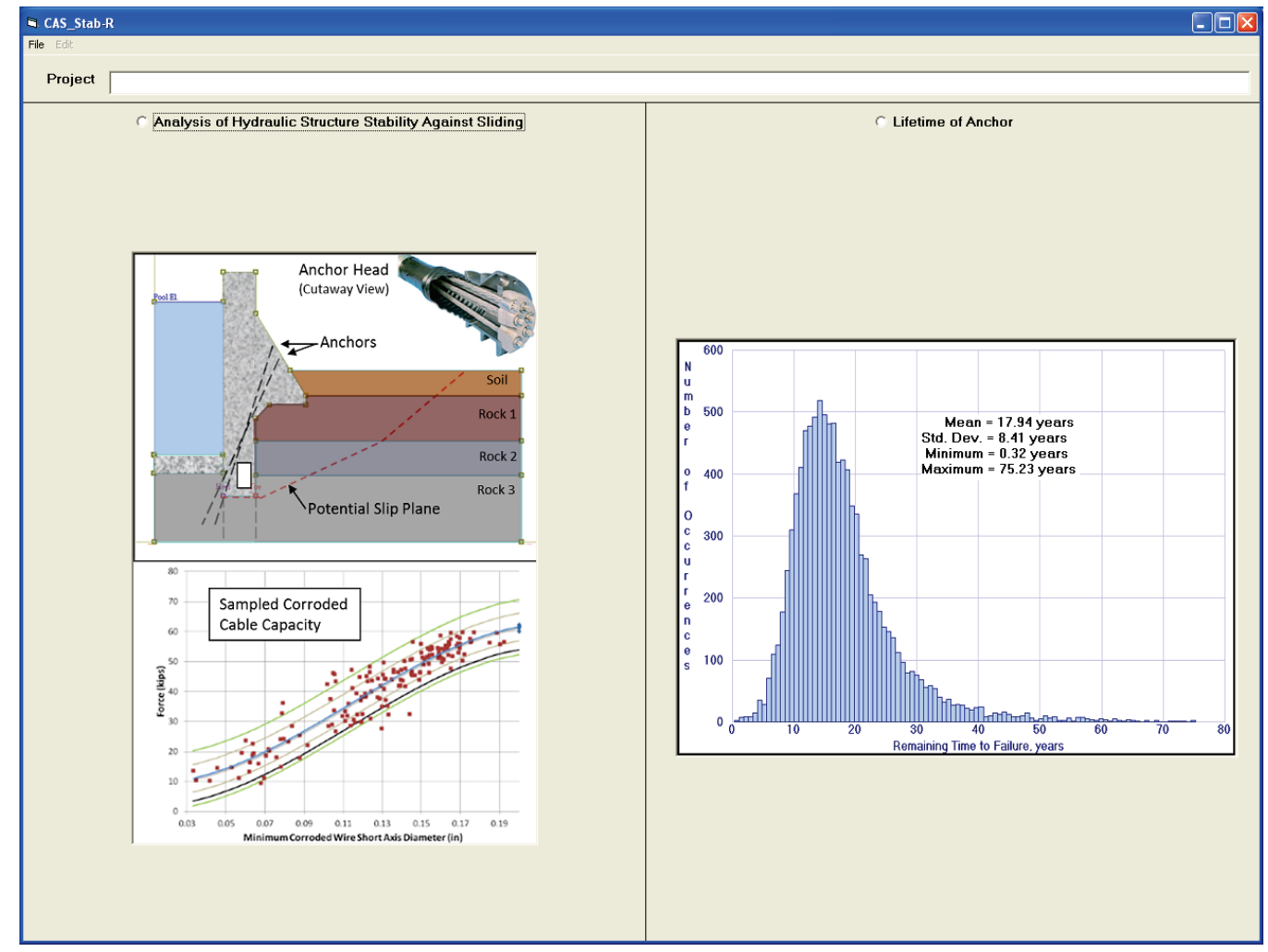


The U.S. Army Engineer Research and Development Center (ERDC) solves the nation's toughest engineering and environmental challenges. ERDC develops innovative solutions in civil and military engineering, geospatial sciences, water resources, and environmental sciences for the Army, the Department of Defense, civilian agencies, and our nation's public good. Find out more at www.erdc.usace.army.mil.

To search for other technical reports published by ERDC, visit the ERDC online library at http://acwc.sdp.sirsi.net/client/default. 


\section{Corroded Anchor Structure Stability/Reliability (CAS_Stab-R) Software for Hydraulic Structures}

Terry W. Warren, Barry C. White, and Robert M. Ebeling

Information Technology Laboratory

U.S. Army Engineer Research and Development Center

3909 Halls Ferry Road

Vicksburg, MS 39180-6199

Final report

Approved for public release; distribution is unlimited.

Prepared for U.S. Army Corps of Engineers

Washington, DC 20314-1000

Under INP Work Unit 33143 


\section{Abstract}

This report describes software that provides a probabilistic estimate of time-to-failure for a corroding anchor strand system. These anchor systems are installed to preserve and extend the service life of U.S. Army Corps of Engineers hydraulic structures.

Corrosion reduces the cross-section area of steel cables until the cable capacity is less than the tension force applied when the anchor cable was initially installed. When enough material is lost from the cable anchor that the cable capacity is less than the lock-off load of the anchor, the anchor will fail and no longer provide stability to the structure. A series of unique pull-test experiments conducted by Ebeling et al. (2016) at the U.S. Army Engineer Research and Development Center provided the required statistical relationships of reduced seven-strand cable capacity to (1) corroded cross-section area and to (2) corroded cross-section minimum short axis diameter for failed cable strands.

The software product $\underline{\text { Corroded }}$ Anchorage $\underline{\text { Structural }} \underline{\text { Stability and }}$ Reliability (CAS_Stab-R) produces probabilistic Remaining Anchor Life time estimates for anchor cables based upon the direct corrosion rate for the installation. CAS_Stab-R can also perform a probabilistic analysis to determine the Probability of Unsatisfactory Performance for a structural model cross section founded on rock.

DISCLAIMER: The contents of this report are not to be used for advertising, publication, or promotional purposes. Citation of trade names does not constitute an official endorsement or approval of the use of such commercial products. All product names and trademarks cited are the property of their respective owners. The findings of this report are not to be construed as an official Department of the Army position unless so designated by other authorized documents. 


\section{Contents}

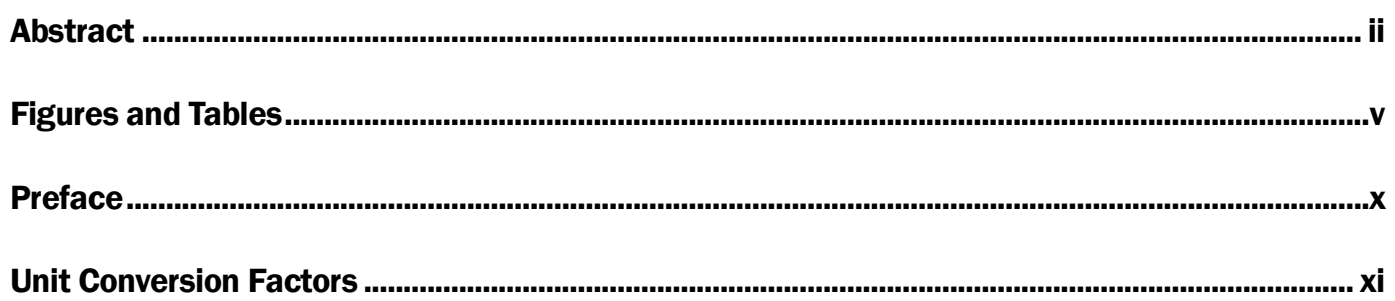

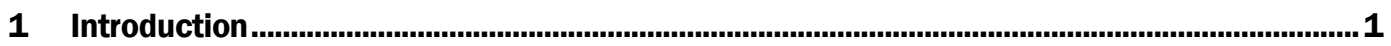

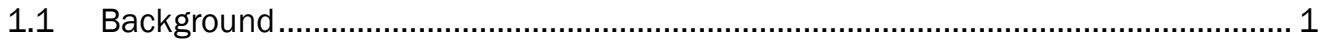

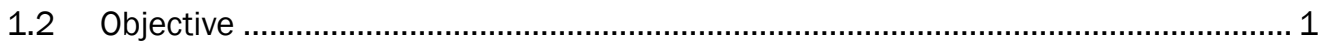

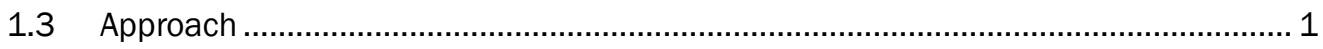

1.4 Report contents ............................................................................................... 3

2 Corroded Anchorage Structural Stability and Reliability Analysis (CAS_Stab-R) Procedural Methods .............................................................................................................. 5

2.1 Remaining Anchor Life (RAL) computation ...................................................... 5

2.1.1 Reduced capacity basis and computation ........................................................ 5

2.1.2 Corrosion variables, distributions, and methods.................................................. 8

2.1.3 Time-to-Failure (TTF) computation ................................................................... 15

2.1.4 Sampling for statistical variability ...................................................................... 16

2.1.5 Summary of inputs and outputs........................................................................ 17

2.2 Structural stability against sliding (Probability of Unsatisfactory

Performance [PUP] Analysis) computations............................................................ 18

2.2.1 Wedge solution overview ................................................................................... 18

2.2.2 Boundary pressure and wedge solution capability for silt and soils.........................22

2.2.3 Reliability methods for calculating the PUP.......................................................... 23

2.2.4 Deterministic solution of Factor of Safety (FOS) .................................................. 31

2.2.5 Advanced First Order Second Moment (ASM) design point determination

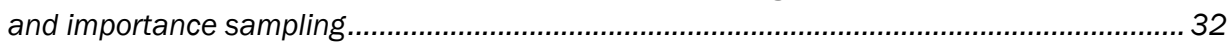

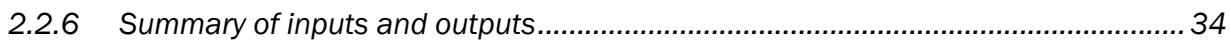

3 CAS_Stab-R Visual Modeler Software ..................................................................................36

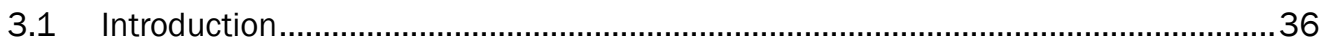

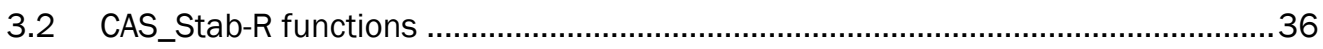

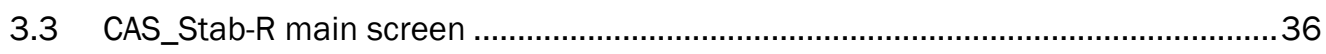

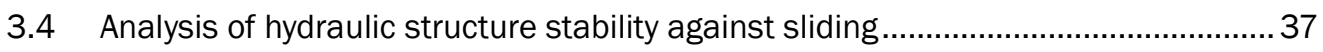

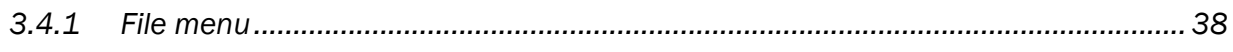

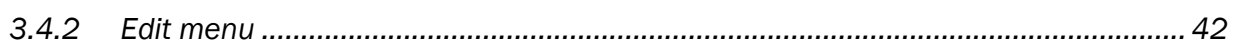

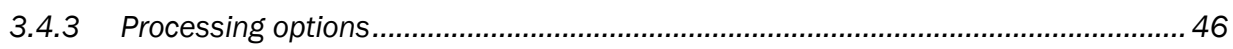

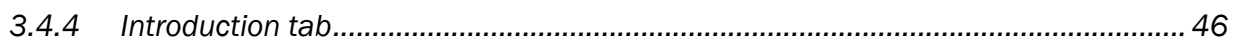

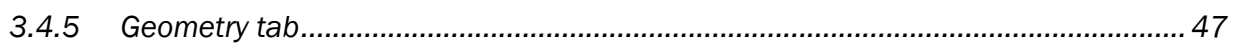

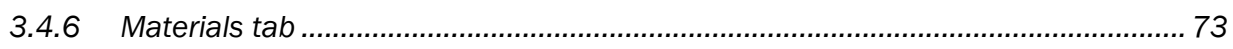

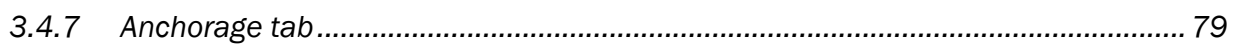

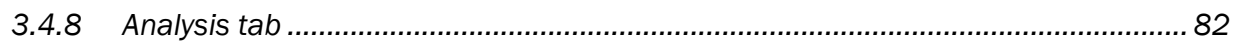




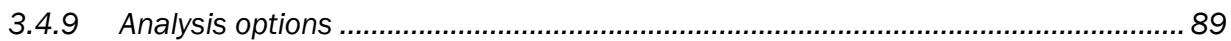

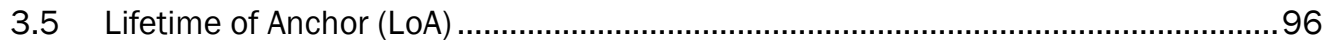

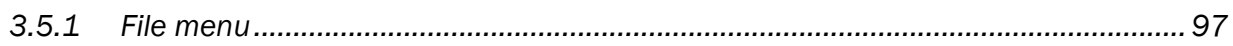

3.5.2 Reduced Anchor Capacity Specification tab ........................................................ 99

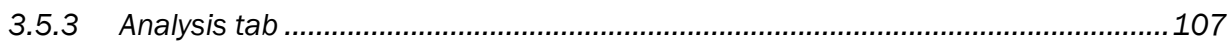

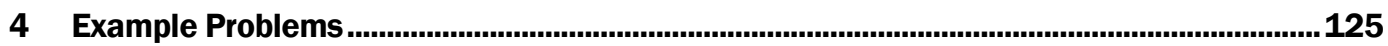

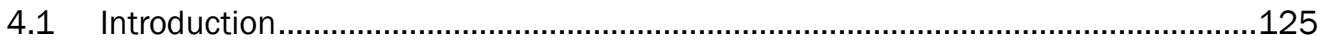

4.2 Example 1 - RAL probabilistic analysis..........................................................125

4.2.1 Quality of dataset input variables - RAL example ............................................... 131

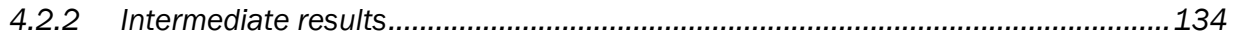

4.3 Example 2 - Stability analysis of a lock structure .............................................. 141

4.3.1 Probabilistic stability analysis of a lock structure .................................................141

4.3.2 Quality of dataset variables - Stability of a lock wall structure example ................154

4.3.3 Effect of number of samples in the dataset on PUP .............................................156

4.3.4 Stability example with RAL example problem settings .........................................157

5 Summary and Conclusions ...................................................................................................... 158

References ...................................................................................................................................... 160

Appendix A: CAS_Stab-R File Formats .................................................................................... 162

Appendix B: CAS_Stab-R Deterministic Solution Examples .......................................................... 176

\section{Report Documentation Page}




\section{Figures and Tables}

\section{Figures}

Figure 1-1. CAS_Stab-R Stability Against Sliding results. .................................................................. 2

Figure 1-2. CAS_Stab-R: Estimated Remaining Anchor Life results...................................................

Figure 2-1. Graph of cable capacity as a function of minimum corroded wire area............................ 6

Figure 2-2. Graph of cable capacity as a function of minimum short axis diameter of the

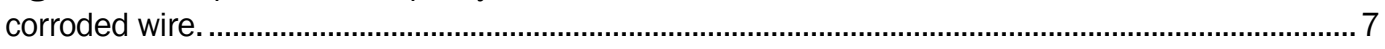

Figure 2-3. Bounded normal probability distribution function. ……...................................................

Figure 2-4. Bounded log normal probability distribution function........................................................

Figure 2-5. Uniform probability distribution function......................................................................10

Figure 2-6. Triangular probability distribution function. ....................................................................10

Figure 2-7. Corrosion effect on cable diameter. ........................................................................12

Figure 2-8. Graph of corrosion rate as a function of dissolved oxygen..............................................13

Figure 2-9. Dispersion variable probability distribution function........................................................15

Figure 2-10. Free body diagram of a theoretical structure...............................................................19

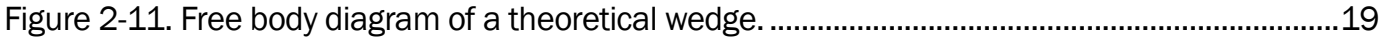

Figure 2-12. Dam free body diagram with silt and soil as boundary pressure forces........................21

Figure 2-13. Free body diagram with slip plane in silt and soil...........................................................23

Figure 2-14. Using the normally distributed regions to assign samples according to the

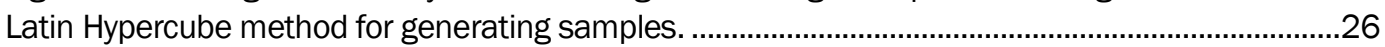

Figure 2-15. For sample $X i$, the distance to the original mean and the distribution mean can be determined and the probabilities computed. ...........................................................................26

Figure 2-16. Finding the position of equivalent cumulative probability for a normal distribution as from a non-normal distribution (after Ang and Tang 1984).......................................30

Figure 3-1. CAS_Stab-R main screen display. ..................................................................................

Figure 3-2. Structural stability Introduction tab. .............................................................................38

Figure 3-3. Structural stability File menu.................................................................................39

Figure 3-4. File Open dialog box................................................................................................

Figure 3-5. File Save As menu option......................................................................................... 41

Figure 3-6. File-Geometry-Print Geometry menu option................................................................ 41

Figure 3-7. Print dialog box...................................................................................................... 42

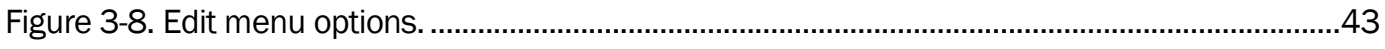

Figure 3-9. Probability Variable Input window. ................................................................................4

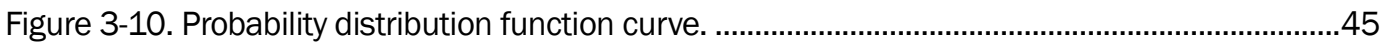

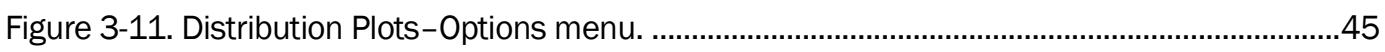

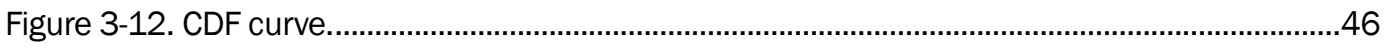

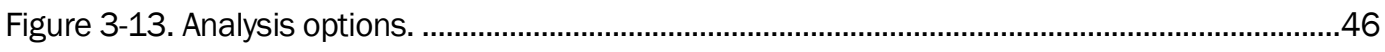

Figure 3-14. CAS_Stab-R structural stability Geometry tab. ……………………………………....4 47

Figure 3-15. Zoom To World button. ……………………...........................................................48 
Figure 3-16. Geometry Display with Zoom Box.......................................................................

Figure 3-17. Geometry Display with Zoomed Area........................................................................49

Figure 3-18. Geometry Display with popup menu. ........................................................................51

Figure 3-19. Node coordinate entry window. .......................................................................

Figure 3-20. Geometry Display with a node at coordinate $(20,50)$................................................52

Figure 3-21. Geometry Display with a highlighted node. ................................................................52

Figure 3-22. Menu option to display node numbers. .....................................................................53

Figure 3-23. Geometry Display with node numbers shown. ...........................................................53

Figure 3-24. Structure node selection message box. ……….....................................................54

Figure 3-25. Geometry Display with partially defined structure. ………………………....................55

Figure 3-26. Geometry Display with a defined structure..................................................................5

Figure 3-27. Select Node as Gallery Location popup option. ...........................................................56

Figure 3-28. Geometry Display with a domed gallery. ....................................................................57

Figure 3-29. Set Node as Top of Gallery Drain popup menu option. ..............................................58

Figure 3-30. Geometry Display with a gallery drain....................................................................60

Figure 3-31. Regions tab. ......................................................................................................61

Figure 3-32. Instructions for defining a material region. .................................................................61

Figure 3-33. Geometry Display with a partially defined region.........................................................62

Figure 3-34. Geometry Display with a completed material region..................................................63

Figure 3-35. Regions tab with the Current Region list box highlighted.............................................63

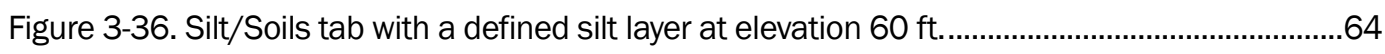

Figure 3-37. Silt/Soils tab after clicking Add New Soil Layer. ......................................................65

Figure 3-38. Model with Soil Layers defined at elevations 60 and $70 \mathrm{ft}$........................................65

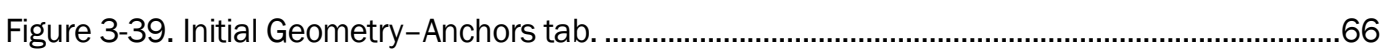

Figure 3-40. Geometry-Anchors tab after clicking Add an Anchor button. ......................................67

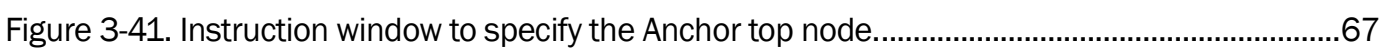

Figure 3-42. Geometry Display with an anchor shown in green.....................................................68

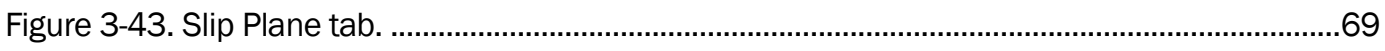

Figure 3-44. Instructions for selecting slip plane nodes.................................................................70

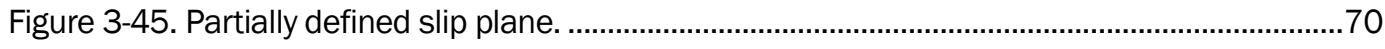

Figure 3-46. Completed potential slip plane. ................................................................................. 71

Figure 3-47. Geometry-Pools tab. ..............................................................................................

Figure 3-48. Resulting display with Headwater Level $=80 \mathrm{ft}$ and Tailwater Level $=70 \mathrm{ft} \ldots \ldots \ldots \ldots \ldots \ldots . . . .72$

Figure 3-49. Initial Materials tab.................................................................................................73

Figure 3-50. Result of clicking the Add a Material button. .............................................................. 74

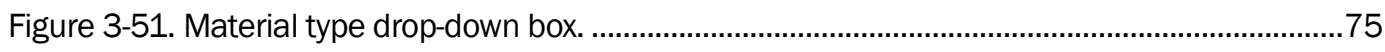

Figure 3-52. K-Type drop-down box.........................................................................................

Figure 3-53. Region - Material Mapping example...........................................................................78

Figure 3-54. Anchorage tab. ....................................................................................................79

Figure 3-55. Anchorage tab with Oxygenation Curve displayed. ...................................................... 81 
Figure 3-56. Initial Analysis tab - probabilistic solution...............................................................82

Figure 3-57. Initial Analysis tab - deterministic solution....................................................................83

Figure 3-58. Determination of ASM Design Point indication. .............................................................85

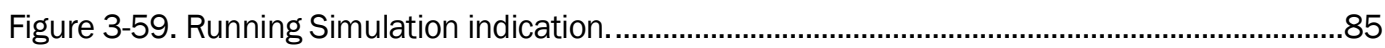

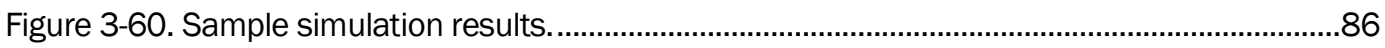

Figure 3-61. Histogram display of an anchor lock-off load input variable..........................................87

Figure 3-62. Text display of an anchor lock-off load probability variable...........................................87

Figure 3-63. Probability Variables Save As dialog box........................................................................8

Figure 3-64. Print dialog box for printing a Probability Variables histogram. ......................................89

Figure 3-65. Flow Options drop-down list..............................................................................................90

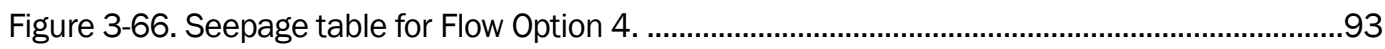

Figure 3-67. Material Stress Type selection box location.................................................................94

Figure 3-68. Soil Solution Method box location. ......................................................................................95

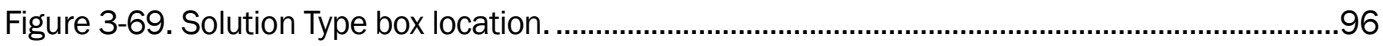

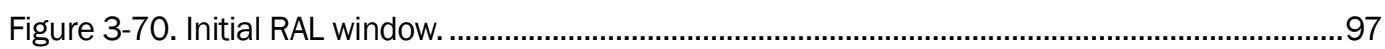

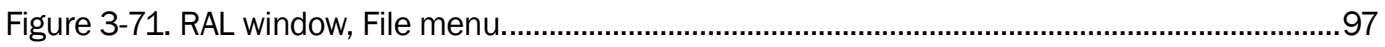

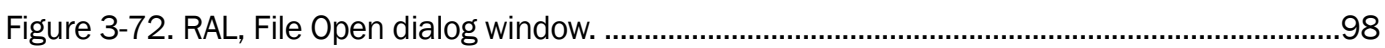

Figure 3-73. RAL File Save As dialog window. ................................................................................99

Figure 3-74. Reduced Anchor Capacity Specification tab with Variables selection frame

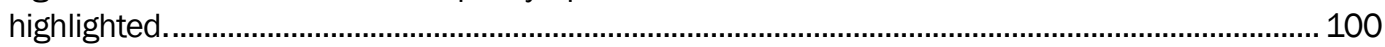

Figure 3-75. Reduced Anchor Capacity Specification tab with highlighted Selected Variable Distribution frame. 101

Figure 3-76. Reduced Anchor Capacity Specification tab with highlighted Select the Corrosion Method frame.

Figure 3-77. Reduced Anchor Capacity Specification tab with highlighted Simplified Average Corrosion Calculation frame..................................................................................... 103

Figure 3-78. Cable failure forces and corresponding minimum corroded wire areas.................... 104

Figure 3-79. Cable failure forces and corresponding minimum short axis diameters................... 104

Figure 3-80. Number of Simulations input box............................................................................. 106

Figure 3-81. Dispersion Variable PDF curve.................................................................................... 106

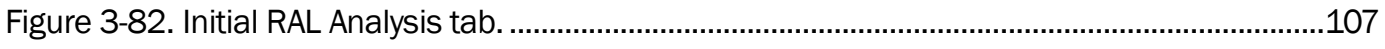

Figure 3-83. Remaining Time To Failure Histogram display.......................................................... 110

Figure 3-84. Remaining Time To Failure PDF plot. ....................................................................... 111

Figure 3-85. Remaining Time To Failure PDF display......................................................................... 112

Figure 3-86. Remaining Time To Failure CDF display................................................................ 112

Figure 3-87. Reduced Anchor Capacity for Reduced Area plot. ...................................................... 113

Figure 3-88. Reduced Anchor Capacity for Reduced Area display. .................................................. 114

Figure 3-89. Lock-off Load for Reduced Area plot. .......................................................................... 115

Figure 3-90. Reduced Anchor Capacity Histogram. ......................................................................... 116

Figure 3-91. Area of Corroded Wire Histogram.......................................................................... 116

Figure 3-92. Lock-off Load Histogram Plot. ......................................................................................117 
Figure 3-93. Corrosion Rate Histogram.............................................................................. 118

Figure 3-94. Oxygenation Level Histogram.......................................................................... 119

Figure 3-95. NDT Corroded Wire Area Histogram....................................................................... 119

Figure 3-96. Dispersion of Reduced Capacity Histogram.......................................................... 120

Figure 3-97. Statistics-Remaining Time to Failure After 10 years Length of Service. ...................... 121

Figure 3-98. LOS frame showing a selection of 10 years............................................................. 122

Figure 3-99. Enabled Print Displayed Results button. .................................................................... 123

Figure 3-100. Print dialog box. .............................................................................................. 123

Figure 3-101. Enabled Save Displayed Results button............................................................... 124

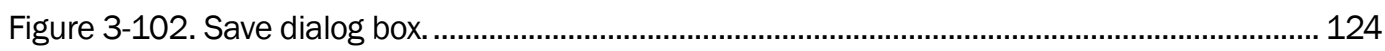

Figure 4-1. Initial Lock-off Force distribution for Example 1....................................................... 126

Figure 4-2. Oxygenation Level selected as the corrosion criteria. .................................................. 126

Figure 4-3. Oxygenation Level distribution assignment............................................................... 127

Figure 4-4. Number of Simulations input window. .................................................................... 127

Figure 4-5. Estimate the Remaining Anchor Life button enabled. ................................................ 127

Figure 4-6. Simplified Average Corrosion Calculation curve selection............................................. 128

Figure 4-7. Remaining Time to Failure Histogram for 0 years LOS for Example 1........................... 128

Figure 4-8. Remaining Time to Failure Histogram for 10 years LOS for Example 1........................ 129

Figure 4-9. Remaining Time to Failure Histogram for 33.3 years LOS for Example 1................... 130

Figure 4-10. Example 1 Remaining Time to Failure CDF plot at LOS of 0 years. ............................ 130

Figure 4-11. Example 1 Lock-off Load Histogram. ………......................................................... 131

Figure 4-12. Example 1 Oxygenation Level Histogram. .............................................................. 132

Figure 4-13. Example 1 Dispersion of Reduced Capacity Histogram............................................ 133

Figure 4-14. Example 1 Reduced Anchor Capacity for Reduced Area graph for LOS = 0

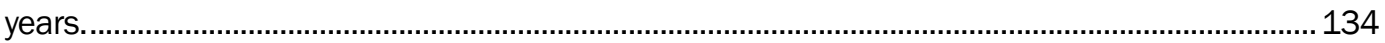

Figure 4-15. Example 1 Reduced Anchor Capacity for Reduced Area graph for LOS $=10$

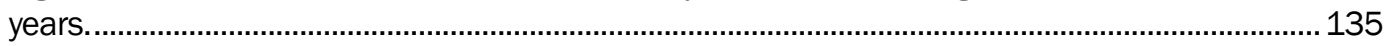

Figure 4-16. Example 1 Reduced Anchor Capacity for Reduced Area graph for LOS $=20$

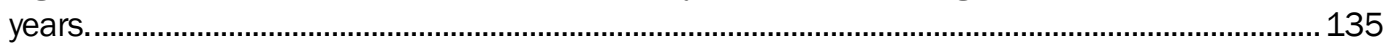

Figure 4-17. Example 1 Lock-off Load for Reduced Area graph for LOS = 0 years. ........................ 136

Figure 4-18. Example 1 Lock-off Load for Reduced Area graph for LOS = 10 years....................... 137

Figure 4-19. Example 1 Lock-off Load for Reduced Area graph for LOS = 20 years...................... 137

Figure 4-20. Example 1 Reduced Anchor Capacity Histogram for LOS = 0 years........................... 138

Figure 4-21. Example 1 Reduced Anchor Capacity Histogram for LOS = 20 years. ........................ 139

Figure 4-22. Example 1 Corroded Wire Area Histogram for LOS = 1 year...................................... 140

Figure 4-23. Example 1 Corroded Wire Area Histogram for LOS $=20$ years................................. 140

Figure 4-24. Lock wall model free body diagram of Example 2 …..................................................141

Figure 4-25. Navigation lock wall model of example problem...................................................... 142

Figure 4-26. Model geometry with displayed node numbers. ...................................................... 144

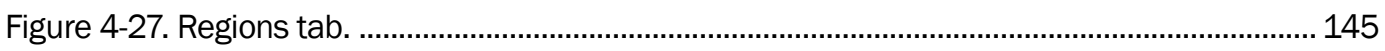

Figure 4-28. Materials tab showing the properties of Material 1................................................. 145 
Figure 4-29. Materials tab showing the properties of Material 2................................................. 146

Figure 4-30. Anchors tab-Definition of lock wall anchors for the example problem..................... 146

Figure 4-31. Pools tab-Definitions for the example problem. ..........................................................147

Figure 4-32. Anchorage tab-Corrosion rate - Oxygenation level curve from Ebeling et al. (2016). 148

Figure 4-33. Anchorage tab with example problem selections for Anchor 1.............................. 148

Figure 4-34. Probability Distributions menu option........................................................................ 149

Figure 4-35. Probability distribution for Oxygenation Level variable...............................................150

Figure 4-36. Probability distribution for Anchor 1 lock-off force variable. ........................................ 150

Figure 4-37. Probability distribution for the Material Number 2 internal friction angle, $\phi . . . . . . . . . . . .151$

Figure 4-38. Initial Analysis tab. .................................................................................................. 152

Figure 4-39. Determining ASM Design Point window....................................................................... 153

Figure 4-40. Running Simulation window................................................................................... 153

Figure 4-41. Analysis tab showing the results of the example problem simulation........................ 153

Figure 4-42. Oxygenation Level distribution. ............................................................................. 154

Figure 4-43. Anchor 1 lock-off force variable distribution. ................................................................. 155

Figure 4-44. Material 2 Phi (internal friction angle) distribution..................................................... 156

Figure B-1. ETL 1110-2-256 (HQUSACE 1981) example geometry. ..................................................176

Figure B-2. Material properties of the four materials utilized in the ETL 1110-2-256 (HQUSACE 1981) example............................................................................................................... 178

Figure B-3. Region - Material Mapping for the ETL 1110-2-256 (HQUSACE 1981) example......... 179

Figure B-4. ETL 1110-2-256 (HQUSACE 1981) example FOS result.................................................. 180

Figure B-5. Modified ETL 1110-2-256 (HQUSACE 1981) example geometry with added gallery, drainage, and anchorage............................................................................................ 181

Figure B-6. Modified ETL 1110-2-256 (HQUSACE 1981) anchorage settings. .............................. 182

Figure B-7. Modified ETL 1110-2-256 (HQUSACE 1981) anchor location. ..................................... 182

Figure B-8. Modified ETL 1110-2-256 (HQUSACE 1981) FOS result. ............................................... 183

\section{Tables}

Table 4-1. Lock wall structure nodes........................................................................................ 143

Table 4-2. PUP values for Number of Samples settings. .............................................................. 157

Table B-1. Node coordinates for the ETL 1110-2-256 (HQUSACE 1981) example..........................177 


\section{Preface}

This report was authorized by Headquarters, U.S. Army Corps of Engineers (HQUSACE), and was compiled from January 2016 through September 2016 under the Navigation Systems Research Program. Charles E. Wiggins, USACE Coastal and Hydraulics Laboratory (CHL), was the Program Manager for the Navigation Systems Research Program. Jeff McKee was the HQUSACE Navigation Business Line Manager.

The research was performed by the USACE Information Technology Laboratory (ERDC-ITL) under the general supervision of Dr. Reed L. Mosher, Director, and Patti S. Duett, Deputy Director. Additional general supervision was provided by Dr. Jerrell R. Ballard, Chief, Computational Science and Engineering Division. W. Jeff Lillycrop (ERDC-CHL) was Navigation Technical Director.

COL Bryan S. Green was Commander, ERDC, and Dr. David W. Pittman was Director. 


\section{Unit Conversion Factors}

\begin{tabular}{|c|c|c|}
\hline Multiply & By & To Obtain \\
\hline cubic feet & 0.02831685 & cubic meters \\
\hline cubic inches & 1.6387064 E-05 & cubic meters \\
\hline cubic yards & 0.7645549 & cubic meters \\
\hline degrees (angle) & 0.01745329 & radians \\
\hline degrees Fahrenheit & $(F-32) / 1.8$ & degrees Celsius \\
\hline feet & 0.3048 & meters \\
\hline foot-pounds force & 1.355818 & joules \\
\hline gallons (U.S. liquid) & $3.785412 \mathrm{E}-03$ & cubic meters \\
\hline hectares & $1.0 \mathrm{E}+04$ & square meters \\
\hline inches & 0.0254 & meters \\
\hline inch-pounds (force) & 0.1129848 & newton meters \\
\hline microinches & 0.0254 & micrometers \\
\hline microns & $1.0 \mathrm{E}-06$ & meters \\
\hline miles per hour & 0.44704 & meters per second \\
\hline mils & 0.0254 & millimeters \\
\hline ounces (mass) & 0.02834952 & kilograms \\
\hline pounds (force) & 4.448222 & newtons \\
\hline pounds (force) per foot & 14.59390 & newtons per meter \\
\hline pounds (force) per inch & 175.1268 & newtons per meter \\
\hline pounds (force) per square foot & 47.88026 & pascals \\
\hline pounds (force) per square inch & 6.894757 & kilopascals \\
\hline pounds (mass) & 0.45359237 & kilograms \\
\hline pounds (mass) per cubic foot & 16.01846 & kilograms per cubic meter \\
\hline pounds (mass) per cubic inch & $2.757990 \mathrm{E}+04$ & kilograms per cubic meter \\
\hline pounds (mass) per square foot & 4.882428 & kilograms per square meter \\
\hline pounds (mass) per square yard & 0.542492 & kilograms per square meter \\
\hline slugs & 14.59390 & kilograms \\
\hline square feet & 0.09290304 & square meters \\
\hline square inches & 6.4516 E-04 & square meters \\
\hline tons (force) & $8,896.443$ & newtons \\
\hline tons (force) per square foot & 95.76052 & kilopascals \\
\hline tons (long) per cubic yard & $1,328.939$ & kilograms per cubic meter \\
\hline
\end{tabular}




\begin{tabular}{|l|c|l|}
\hline Multiply & By & To Obtain \\
\hline tons (2,000 pounds, mass) & 907.1847 & kilograms \\
\hline tons (2,000 pounds, mass) per square foot & $9,764.856$ & kilograms per square meter \\
\hline yards & 0.9144 & meters \\
\hline
\end{tabular}




\section{Introduction}

\subsection{Background}

The U.S. Army Corps of Engineers (Corps) is responsible for the operation and maintenance of a large inventory of concrete navigation structures. Over the last 40 years, a number of existing Corps hydraulic structures have been retrofitted with post-tensioned, multi-strand cable anchorage systems to prevent movement in the form of sliding or overturning, thereby enhancing the stability of these structures. Methods of corrosion resistance applied to the anchorages have evolved and improved over time. However, older retrofits have resulted in multi-strand anchor cables that have experienced corrosion due to a lack of proper corrosion mitigation procedures utilized at the time of installation.

The capacity of a cable decreases as steel material is lost from the strands of the cable due to corrosive processes. When the multi-strand anchor cables lose enough material to reduce the capacity of the cable below the tension force that was applied at installation, the anchor will fail, thereby reducing the stability of the structure. A series of unprecedented pull-test experiments conducted at the Engineer Research and Development Center (ERDC) with results published by Ebeling et al. (2016) and Haskins et al. (2016b) provided statistical relationships of reduced cable capacity to the reduced cross-section area and to the cross-section minimum short axis diameter of failed cable strands with varying levels of corrosion.

\subsection{Objective}

The objective of this research is to develop software that provides a probabilistic estimate of time-to-failure for a corroding anchor strand system. These anchor systems of interest are those that have been installed to preserve and extend the service life of Corps hydraulic structures.

\subsection{Approach}

Recent development work at the ERDC has produced a software product

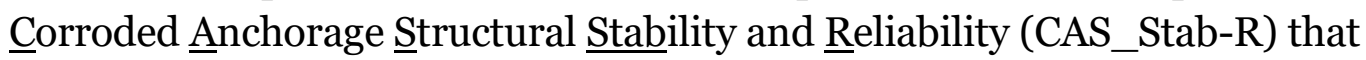
incorporates (1) the knowledge gained from results of the ERDC pull-test experiments on corroded cable strands and (2) the wedge solution technique of ETL 1110-2-256 (HQUSACE 1981) for assessing the stability of 
a hydraulic structure against sliding. The wedge solution software performs a probabilistic analysis to determine the Probability of Unsatisfactory Performance (PUP) for a structural model cross section founded on rock. Figure 1-1 shows example output with a resulting PUP value of 0.95 for a structural model supported by corroding anchorage. The corrosion is caused by moisture with a mean oxygenation level of 1.175 parts per million (ppm) in the immediate anchorage environment. The PUP is computed for the structure and corroding anchor system after a Length of Service (LOS) time of 33.3 years.

Figure 1-1. CAS_Stab-R Stability Against Sliding results.

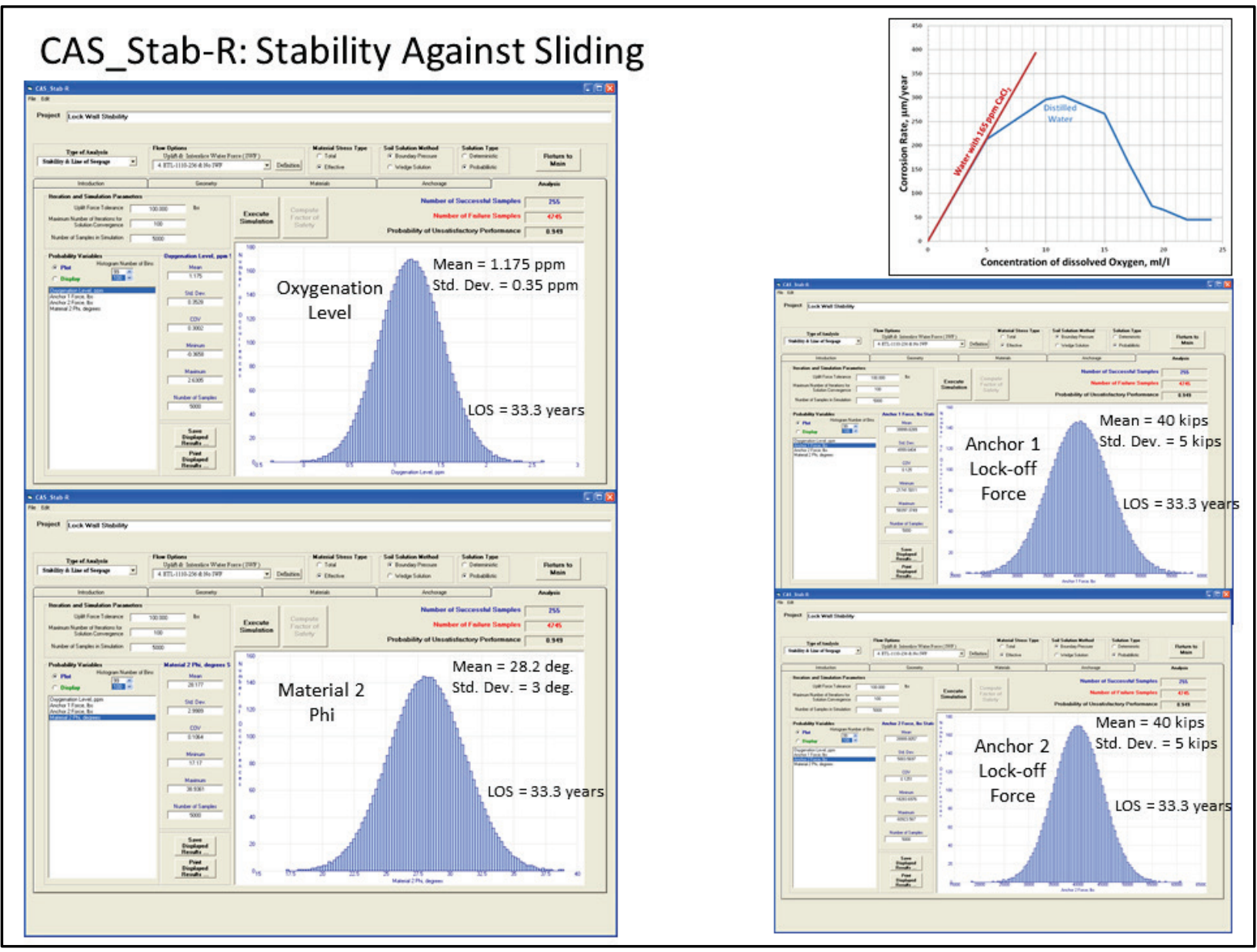

CAS_Stab-R can also produce probabilistic Remaining Anchor Life (RAL) estimates for anchor cables based upon the direct corrosion rate for the installation. Figure 1-2 shows example output results for a structural model containing corroding anchorage, resulting in a mean RAL of 17.94 years for an anchor with the same mean 1.175 ppm oxygenation level environment distribution that was discussed in the previous paragraph. 
This report provides the engineering basis for computations performed by CAS_Stab-R, a user manual for the CAS_Stab-R Visual Modeler, and sample problems showing the use of CAS_Stab-R.

Figure 1-2. CAS_Stab-R: Estimated Remaining Anchor Life results.

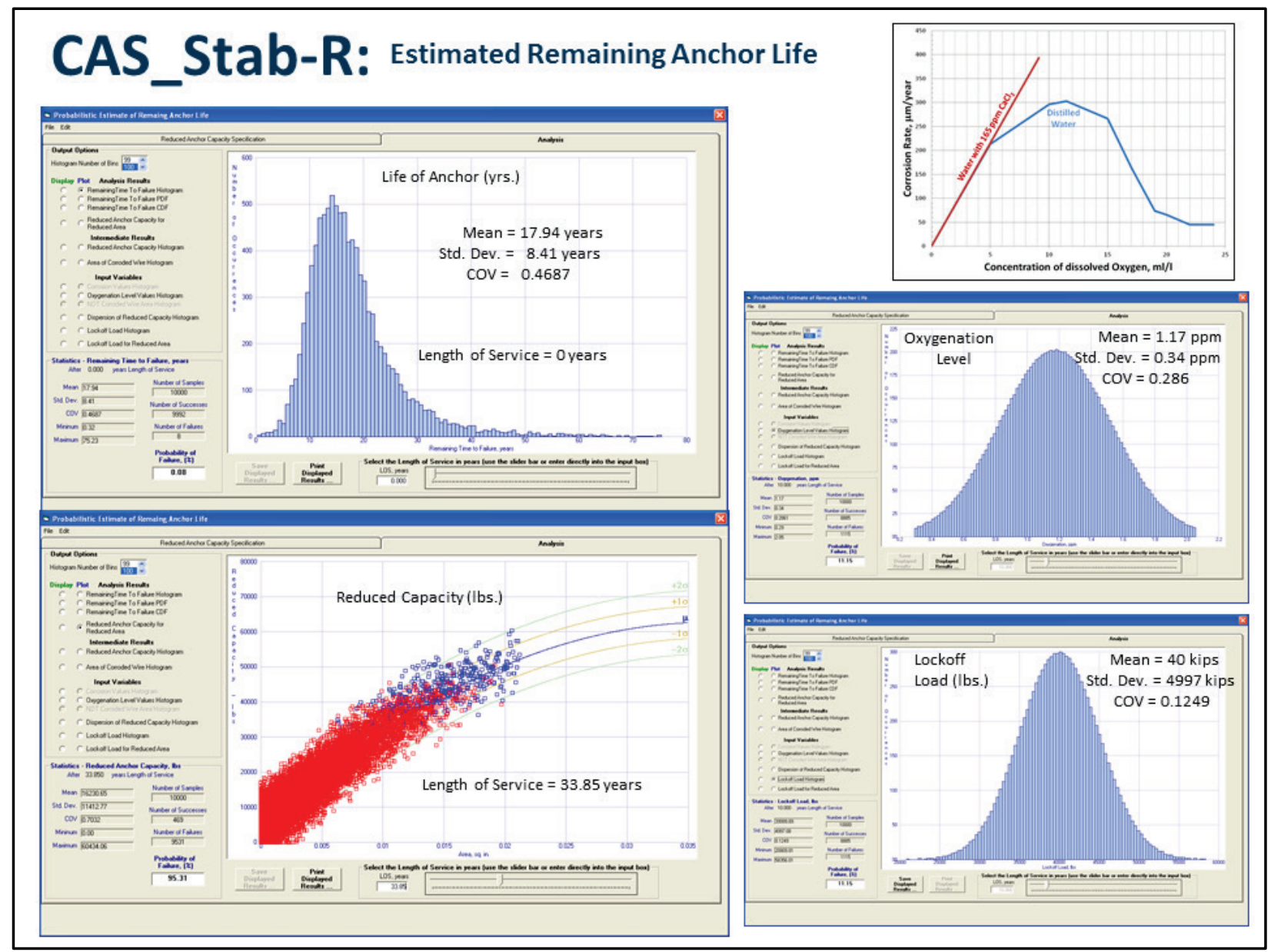

\subsection{Report contents}

Chapter 2 discusses the corroded cable pull-test data results and their application in CAS_Stab-R. The specification of variables and the computations for the probabilistic RAL results are discussed. The equations used to compute the Factor of Safety (FOS) for a two-dimensional (2-D) cross-section model of a structure are also presented. The variables and procedure for a statistical simulation to produce probabilistic PUP results is detailed. The statistical simulation procedures start with performing an Advanced First Order Second Moment (ASM) calculation to establish the Importance Sampling parameters for the Latin Hypercube Monte Carlo simulations. The simulations are then run to determine the PUP value. 
Chapter 3 discusses the CAS_Stab-R Visual Modeler. User input and the output capabilities are presented.

Chapter 4 presents two sample problem results obtained with CAS_Stab$\mathrm{R}$. The first sample problem is an RAL problem with the corrosion rate determined by the knowledge of the oxygenation level of the anchor cable environment. The second sample problem is a simulation for a PUP analysis for a lock wall with dual anchorages.

Chapter 5 summarizes the results of the CAS_Stab-R capabilities.

Appendix A discusses the file formats of the input and output files produced by CAS_Stab-R.

Appendix B presents two case study problems and their CAS_Stab-R solution. The first is the sample five-wedge solution problem of ETL 1110-2-256 (HQUSACE 1981). The second is the same five-wedge geometry with the addition of an anchorage system and a gallery drainage system. 


\section{Corroded Anchorage Structural Stability and Reliability Analysis (CAS_Stab-R) Procedural Methods}

The CAS_Stab-R software incorporates the corroded cable reduced capacity information presented in Ebeling et al. (2016) to compute probabilistic assessments of the expected remaining lifetime of structural anchor cables and the PUP of a navigation structure that employs posttensioned stranded wire cable anchorages. The wedge solution methodology of Engineering Technical Letter (ETL) 1110-2-256 (HQUSACE 1981) is utilized to compute the FOS results, which provide the PUP solution.

\subsection{Remaining Anchor Life (RAL) computation}

\subsubsection{Reduced capacity basis and computation}

A series of experiments were conducted at the ERDC in 2013 to measure the loading capacity of seven-strand wire cables. The laboratory experiments and statistical processing of the resulting (reduced) strength data were discussed in Ebeling et al. (2016) and summarized in Haskins et al. (2016b). The seven strands were arranged with a center "king" wire surrounded by six "outer" wires that spiraled along the length of the king wire. The average king wire diameter was 0.2 inch (in.). The average outer wire diameter was 0.198 in. Twenty-two cable specimens were in pristine condition while 161 specimens were subjected to various levels of corrosion. Corroded specimens contained wire strands with minimum areas that ranged from 0.0015 to 0.0288 square inches (in. ${ }^{2}$ ) and short axis diameters that ranged from 0.033 to $0.192 \mathrm{in}$. Capacity was determined by tension loading of the specimen until failure occurred in one or more wire strands. The capacity was the ultimate tensile load before wire failure. Two size measurements of the failure strand were recorded using optical scanning for morphological properties. The first measurement was the cross-sectional area of the wire strand at the point of failure. The second measurement was the short axis diameter of the most corroded wire at the point of failure.

A scatter plot of the sampled cable capacities as a function of the minimum cross-sectional area is shown in Figure 2-1. As discussed in Ebeling et al. (2016), the minimum area of the smallest wire in the seven-strand 
assembly was used to correlate to the reduced strength of the corroded seven-strand wire assemblage. Statistical analysis of the cable capacity as a function of the corroded wire area (of the smallest wire) yielded the second order equation for the mean trend line given in Equation 2.1.

Figure 2-1. Graph of cable capacity as a function of minimum corroded wire area.

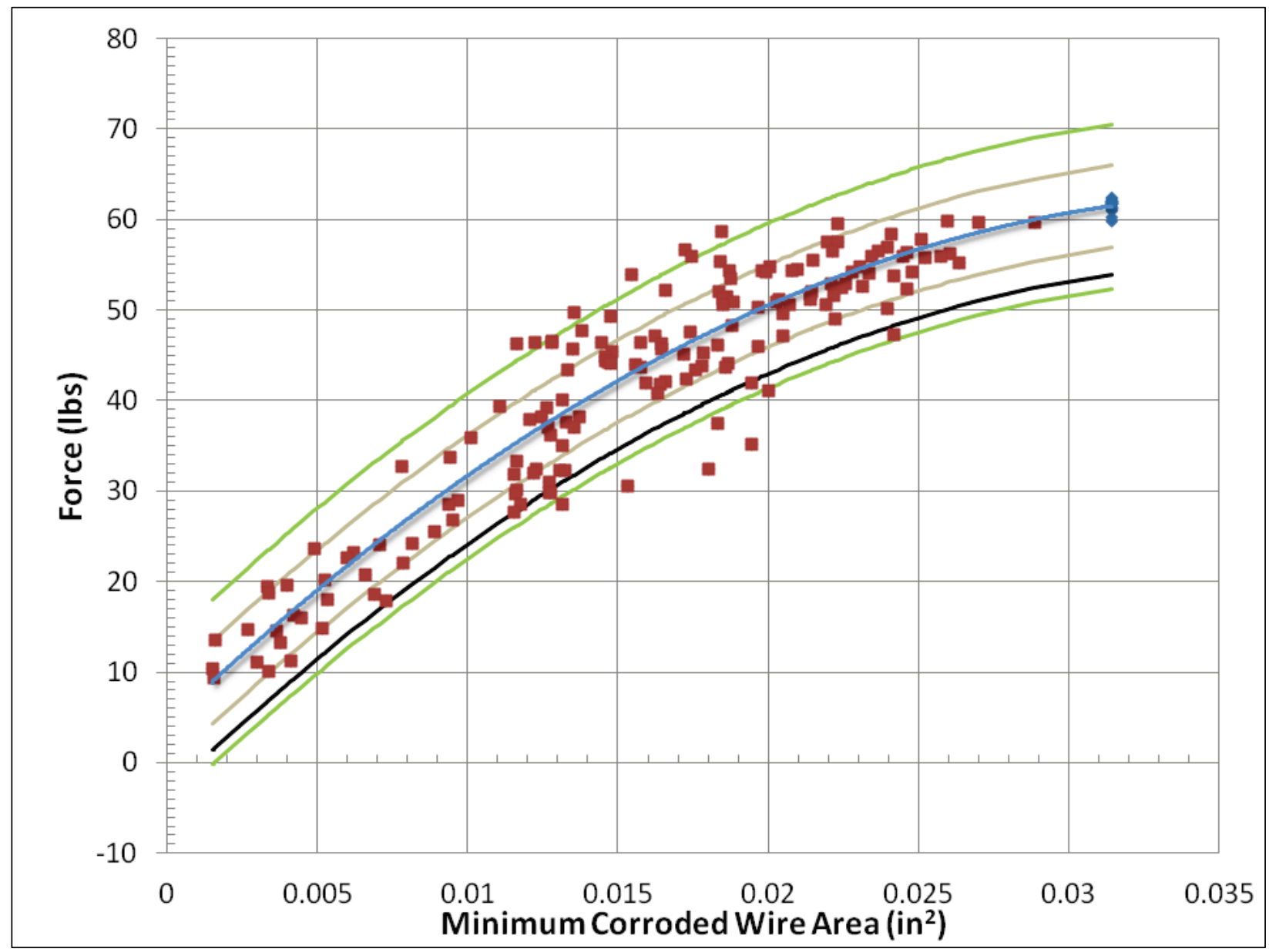

$$
C=-43087028.19 * A^{2}+3176464.38 * A+4175.65
$$

where:

$C=$ the mean cable capacity, in pound force

$A=$ the area of the most corroded wire, in square inches.

The blue line in Figure 2-1 is the graph of the function given in Equation 2.1. The brown lines represent plus and minus one standard deviation from the mean function (Equation 2.1). The green lines represent plus and 
minus two standard deviations from the mean function. The standard deviation of the data about the trend line is 4,557 pounds (lb).

A scatter plot of the cable capacity as a function of the short axis diameter of the most corroded wire is shown in Figure 2-2. Statistical analysis of the cable capacity as a function of the short axis diameter yielded the third-order equation for the mean trend line given in Equation 2.2.

Figure 2-2. Graph of cable capacity as a function of minimum short axis diameter of the corroded wire.

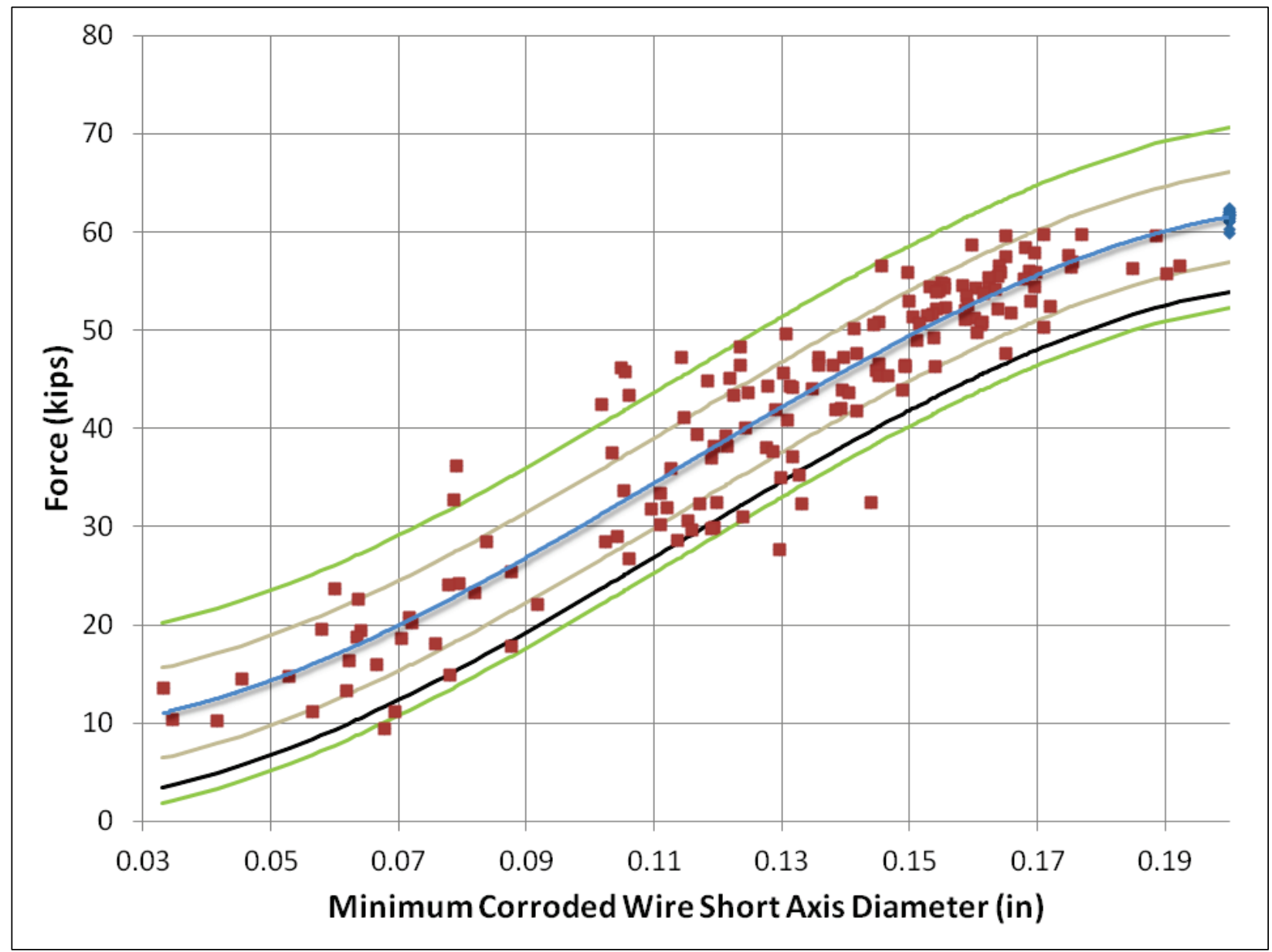

$$
C=-12,418,355.96 * d^{3}+4,237,505.61 * d^{2}-92,773.5 * d+9,918.59
$$

where:

$C=$ the mean cable capacity, in pounds

$d=$ the short axis diameter of the most corroded wire, in inches. 
The blue line in Figure 2-2 is the graph of the function given in Equation 2.2. The brown lines represent plus and minus one standard deviation from the mean function. The green lines represent plus and minus two standard deviations from the mean function. The standard deviation of the data about the trend line is $4,589 \mathrm{lb}$.

CAS_Stab-R provides the user the option of utilizing either Equation 2.1 or 2.2 to predict the cable capacity based on the corroded area or short axis diameter, respectively, of the anchor cable.

\subsubsection{Corrosion variables, distributions, and methods}

Stranded cable anchorage systems providing stability to navigation structures experience corrosive effects that, over time, can lead to cable failure. The parameters that affect the lifetime of the cable are the lock-off load applied to the cable and the rate of cable corrosion. The capacity of a cable decreases as the cable loses material due to corrosion. When the cable capacity falls below the lock-off load applied, the cable will fail, reducing the stability of the structure.

CAS_Stab-R provides the capability to allow uncertainty of these parameters in the determination of the RAL of a cable. The uncertainty is expressed as a distribution type and the parameters necessary to define the type of distribution. The distribution types supported for the RAL computations are bounded normal, bounded log normal, uniform, and triangular.

A bounded, sometimes referred to as truncated, normal distribution of values is a normalized Gaussian distribution that has upper and lower limits on the allowable values. A typical normalized bounded normal distribution is shown in Figure 2-3. A bounded normal distribution is defined by the mean value, standard deviation value, lower bound, and upper bound.

A bounded lognormal distribution of values is a distribution in which the logarithm of the value is a normal distribution. A typical normalized bounded lognormal distribution is described in Figure 2-4. A bounded lognormal distribution is defined by the mean value, standard deviation value, lower bound, and upper bound.

A uniform distribution is defined by a lower bound and an upper bound on values. A typical normalized uniform distribution is shown in Figure 2-5. 
Figure 2-3. Bounded normal probability distribution function.

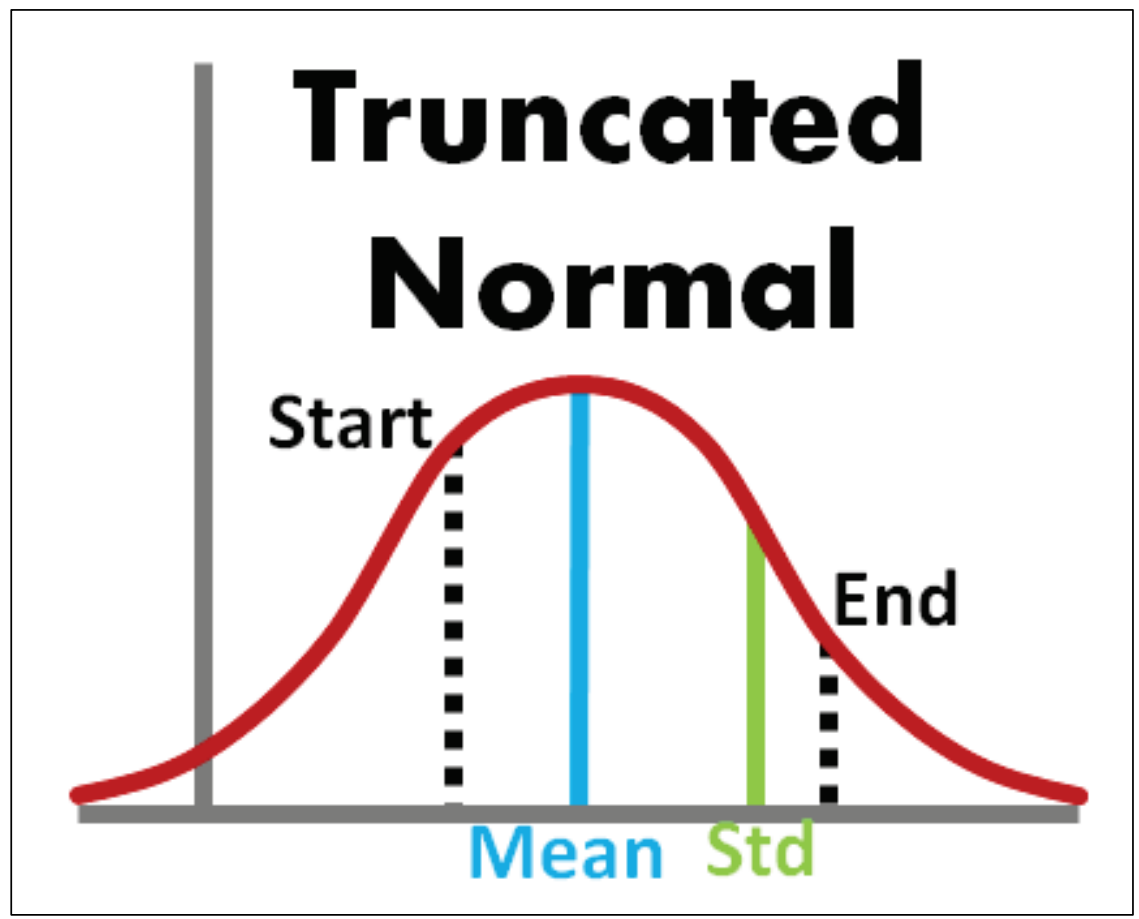

Figure 2-4. Bounded log normal probability distribution function.

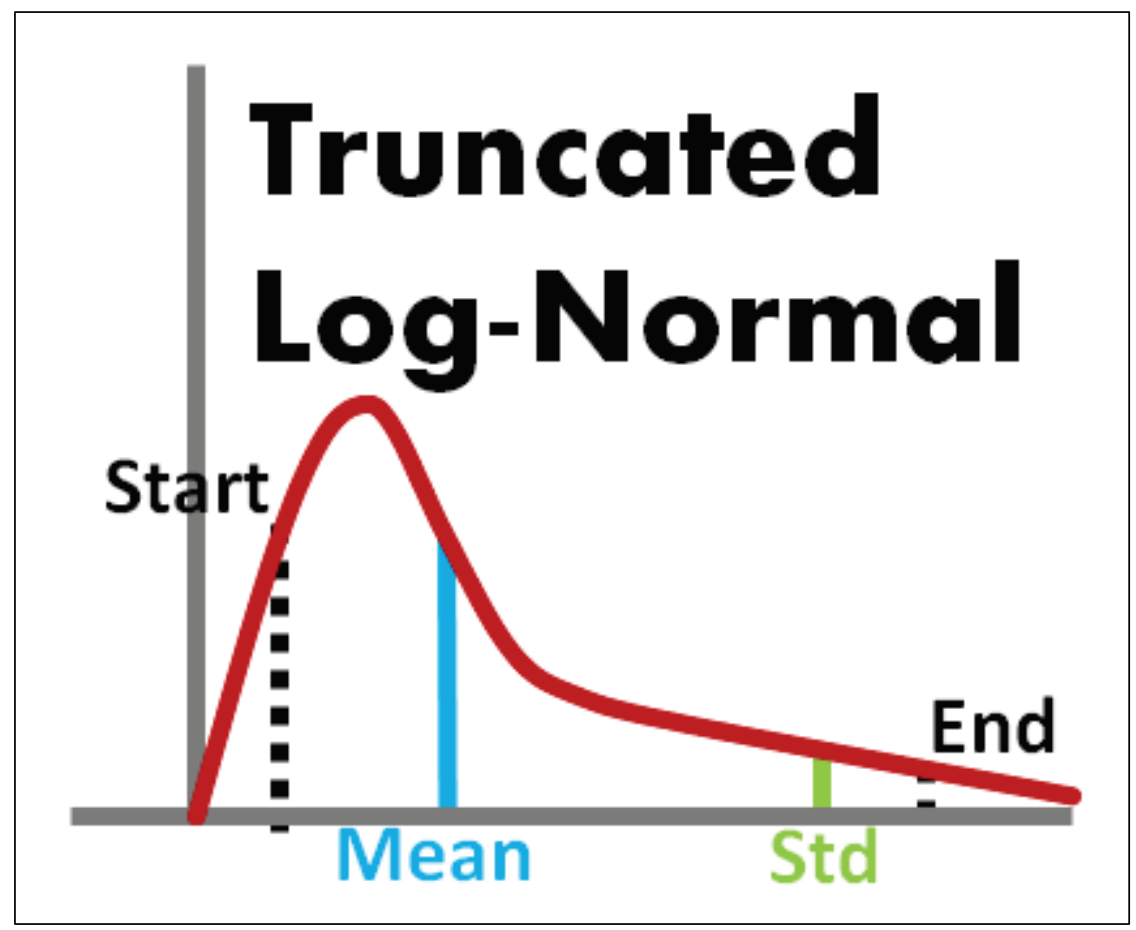


Figure 2-5. Uniform probability distribution function.

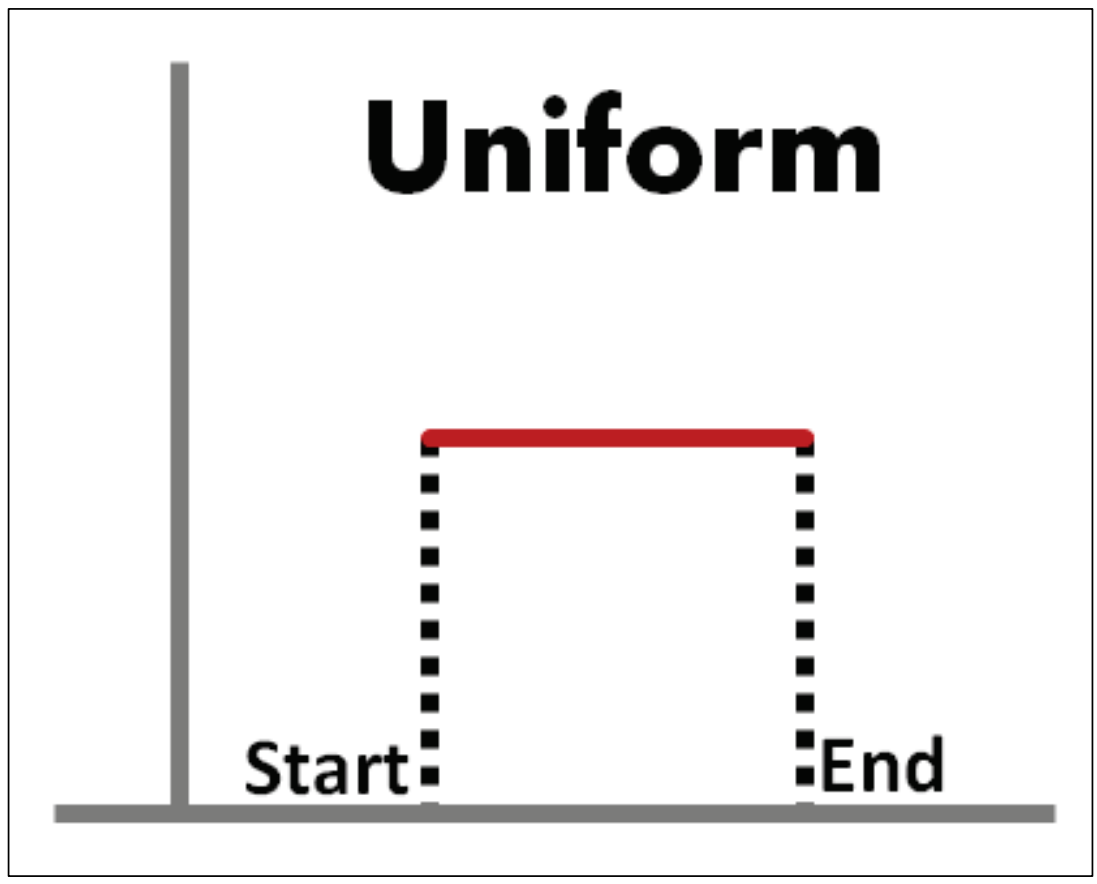

A triangular distribution is defined by a lower bound, an upper bound, and a mid-point. A typical normalized distribution is shown in Figure 2-6.

Figure 2-6. Triangular probability distribution function.

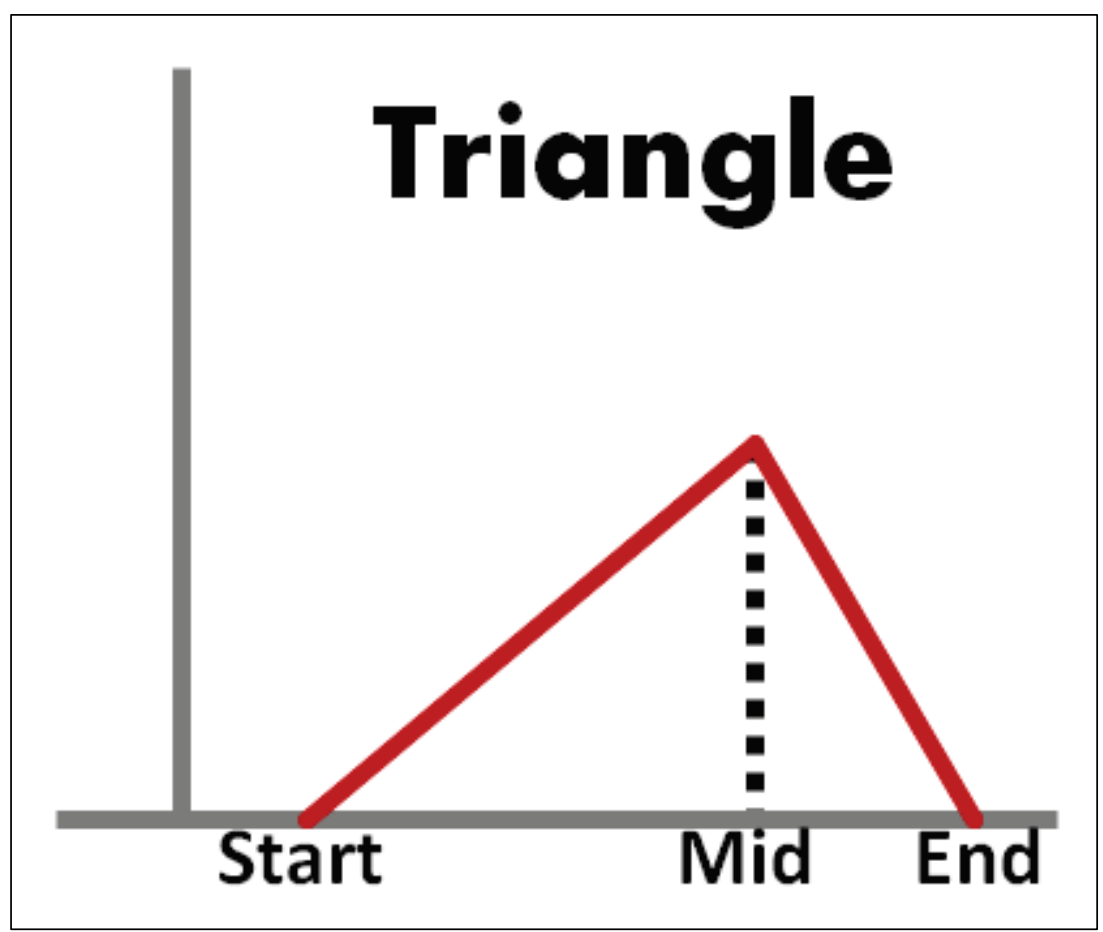




\subsubsection{Lock-off loads}

One end of anchor cables in navigation structures is bonded in place in the foundation material at an elevation below the potential slip plane surface. The cables then have a tension force applied and are locked off at their upper ends to anchor the structure to the foundation. The tension force applied is referred to as the lock-off load. Due to uncertainties in the application of the tension force and naturally occurring relaxation in cables, the lock-off load is considered a variable in CAS_Stab-R.

\subsubsection{Cable corrosion}

The level of corrosion in a cable can be predicted in various ways. One way is a direct physical measurement of an exposed anchor cable under comparable conditions. Combining the measurement with the length of time the cable has been in service, the corrosion rate for the cables can be estimated. Another way is to use knowledge of the environment and quality of the installed corrosion protection applied to the anchor. Yet another way is a Nondestructive Test (NDT) to determine the present anchor cable size.

Given the corrosion rate, oxygenation level, or an NDT measurement and the length of service (LOS) of the cables, CAS_Stab-R can perform a probabilistic determination of the existing cable capacity. The capacity of the corroded cable is computed using either of the user-selected curves of Figures 2.1 or 2.2.

\section{User-Defined Corrosion Rate corrosion specification}

If the user has knowledge of the corrosion rate for an anchorage system, The User-Defined Corrosion Rate option is selected, and the user enters the corrosion rate. The uncertainty in the specified corrosion rate is reflected in the mean and standard deviation values given for the corrosion rate. The computations for the reduced capacity is dependent upon the selected reduced cable capacity curve selected (Figure 2-1 or Figure 2-2). The following two sections explain the computations for the two curves.

\section{Corroded wire area curve}

The diameter of the corroded cable is computed as the difference between the average pristine wire diameter and twice the product of the corrosion 
rate with the LOS. The corrosion is multiplied by 2 as corrosion is assumed to occur around the entire diameter of the cable strand as illustrated in Figure 2-7. The figure shows that as material corrodes from around the cable strand, the diameter is reduced on each "side" of the cable strand necessitating the factor-of-2 multiplication.

Figure 2-7. Corrosion effect on cable diameter.

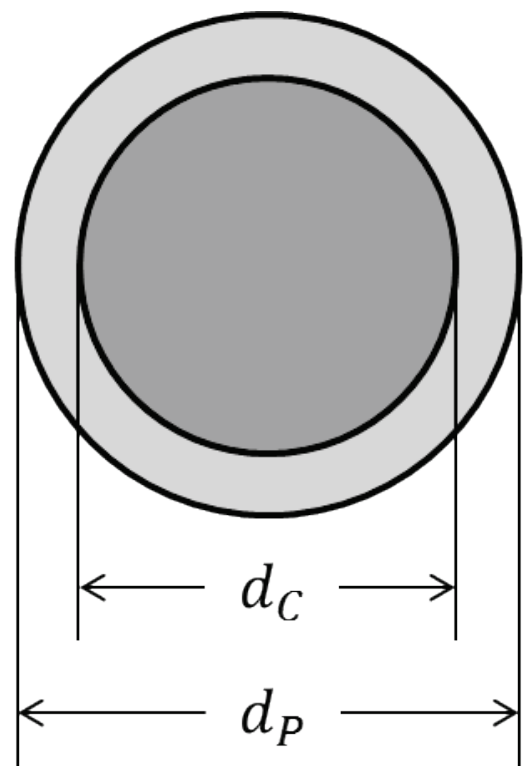

The average pristine wire diameter is the smaller of the king wire and the outer wire diameters. In Equation 2.3, $d_{C}$ is the diameter of the corroded cable, $d_{P}$ is the diameter of the pristine cable, $R$ is the user-supplied corrosion rate, and LOS is the length of the time the anchor has been in service.

$$
d_{C}=d_{P}-2 * R^{*} L O S
$$

The corroded cable area is computed using the standard equation for the area of a circle. In Equation 2.4, $A_{C}$ is the area of the corroded cable and $d_{C}$ is the corroded cable diameter from Equation 2.3.

$$
A_{C}=\pi^{*} d_{C}^{2} / 4
$$

$A_{C}$ is substituted for $A$ in Equation 2.1 to obtain the capacity of the corroded seven-strand wire assemblage. 
Corroded wire short axis diameter curve

The diameter of the corroded cable is computed as above in Equation 2.3. The diameter $d_{C}$ is substituted for $D$ in Equation 2.2 to obtain the capacity of the corroded seven-strand wire assemblage.

\section{Oxygenation concentration corrosion specification}

The second method to compute the reduced capacity of the cable is to specify the oxygen concentration in the cable environment. A relationship between the concentration of oxygen surrounding the cable and the rate of corrosion has been shown in Ebeling et al. (2016). Figure 2-8 contains the curve that illustrates the relationship. As anchorages are not installed in a distilled water environment, the curve for water with a concentration of $165 \mathrm{ppm} \mathrm{CaCl}_{2}$ is used to compute a corrosion rate from the supplied oxygenation concentration. Based on this curve, a conversion factor of 43.25 (microns/year)/ppm was determined. This scale factor is the slope of the red line in Figure 2-8. After the supplied oxygenation concentration is multiplied by the 43.25 conversion factor, the resulting corrosion rate is used to determine the corroded cable capacity as shown above.

Figure 2-8. Graph of corrosion rate as a function of dissolved oxygen.

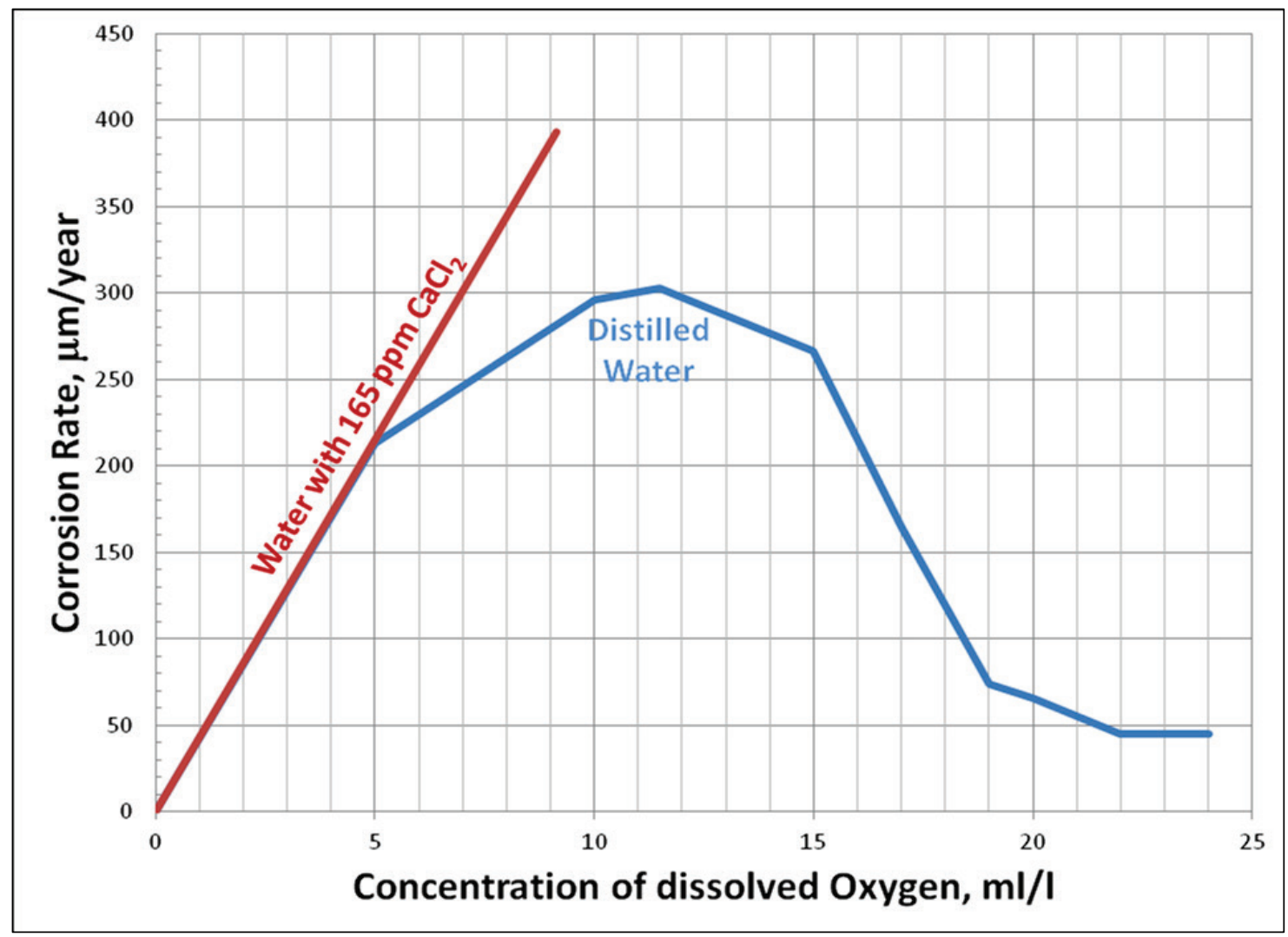




\section{NDT measurement for corroded cable size}

If an NDT measurement of the corroded cable size is available, the corroded cable capacity can be determined by substituting the NDT measurement in Equation 2.1 for an NDT area measurement or Equation 2.2 for an NDT diameter measurement. The authors are unaware of any currently available NDT methods to obtain this measurement, but CAS_Stab-R includes this capability as research in this area is ongoing (e.g., Haskins et al. 2016a).

\subsubsection{Dispersion variable for measured anchor capacities}

The computation of the corroded cable capacity detailed in the previous section is made with Equation 2.1 or 2.2 depending on the designer's selection. The computed cable capacity from these equations will always yield a point on the mean (blue) curve shown in Figures 2-1 or 2-2. As seen in the figures, the pull-test data results contain uncertainties, points above and below the blue capacity line. The standard error for the data in Figure 2-1 is 4,557 lb. The standard error for the data in Figure 2-2 is 4,589 lb. CAS_Stab-R implements this uncertainty into the corroded cable capacity by the introduction of a dispersion variable. The samples (the use of samples for statistical variability is discussed in section 2.1.4) for the dispersion variable are (randomly) generated as a truncated, normalized normal distribution with a mean value of 0.0, a standard deviation of 1.0, minimum value of -3.0, and maximum value of 3.0 as shown in Figure 2-9. The lower bound of -3.0 and upper bound of 3.0 provides a probability density function that includes $99.73 \%$ of the values of an unbounded normal distribution.

The randomly generated dispersion variable value is multiplied by the appropriate standard error, 4,557 lb for the corroded wire area data or 4,589 $\mathrm{lb}$ for the short axis diameter data, and added to the value obtained from the mean trend line Equations 2.1 and 2.2. The use of this variable produces reduced capacities with the same variance as the pull-test data sets. 
Figure 2-9. Dispersion variable probability distribution function.

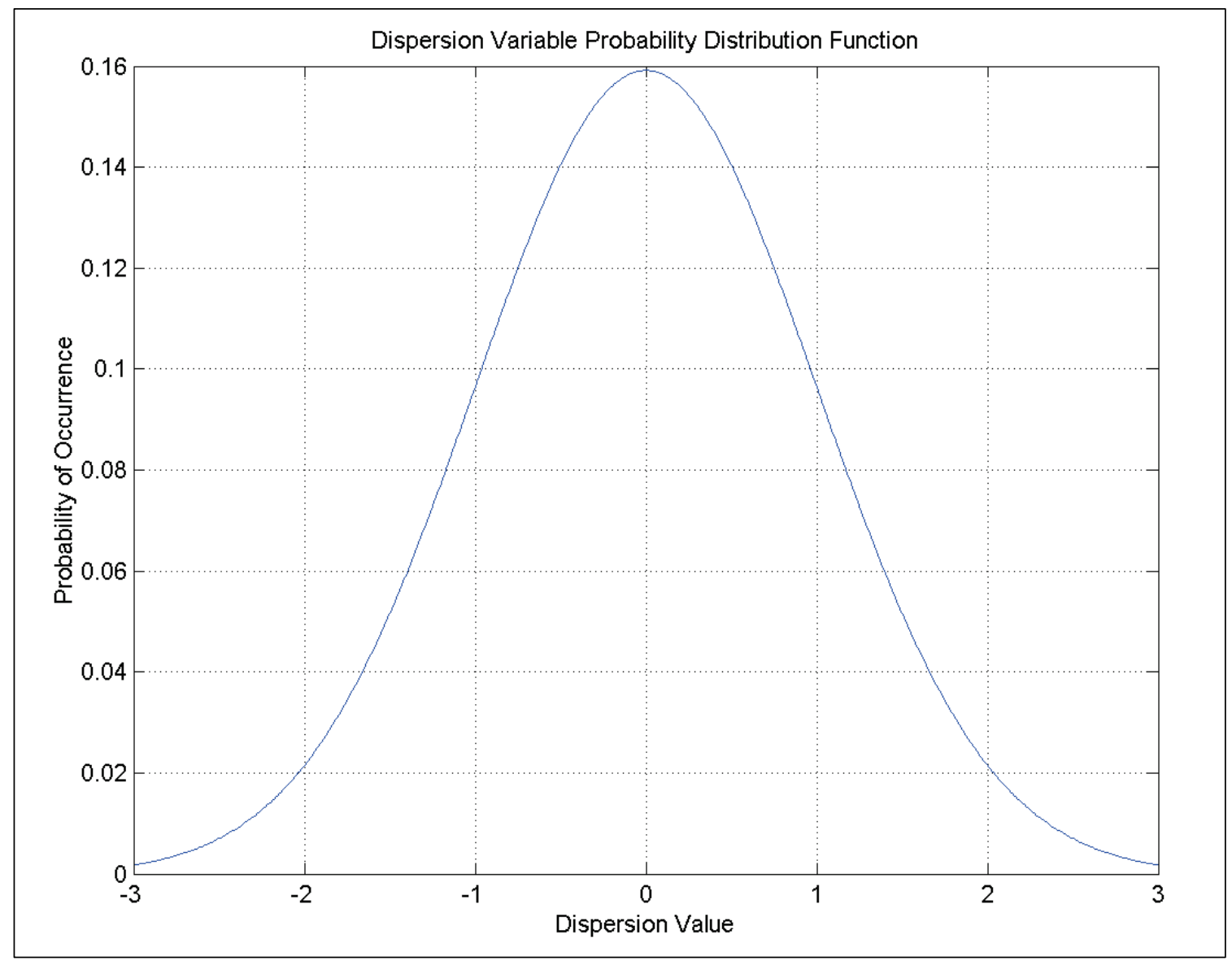

\subsubsection{Time-to-Failure (TTF) computation}

The TTF for a cable is the length of time required for the cable to corrode down to a size for which the lock-off load force exceeds the capacity of the cable. CAS_Stab-R performs the TTF computation in the following manner dependent upon the capacity curve chosen.

\subsubsection{Corroded wire area curve}

The corroded cable area that provides the capacity to support the lock-off load force is computed by assigning $C$ in Equation 2.1 to the lock-off load and solving the equation for $A$. Equation 2.1 is a quadratic equation, so $A$ is computed using the quadratic formula as shown in Equation 2.5. $F_{L O L}$ in Equation 2.5 is the lock-off load force.

$$
A=\frac{-3176464.38 \pm \sqrt{3176464.38^{2}-4(-43087028.19)\left(4175.65-F_{L O L}\right)}}{2(-43087028.19)}
$$


The diameter, $d_{L O L}$, for a circle with the area $A$ is computed as in Equation 2.6 to obtain the diameter of the corroded cable that equals the lock-off load capacity.

$$
d_{L O L}=\sqrt{\frac{4 A}{\pi}}
$$

The TTF is computed by first computing the difference between the current corroded cable diameter found in Equation 2.3 and the lock-off load force diameter.

The diameters are compared. If the corroded cable diameter is less than the lock-off load force diameter, then the TTF is set to zero as the corroded cable will have already failed. If the corroded cable diameter is greater than lock-off load force diameter, the difference is divided by the corrosion rate to obtain the length of time until the cable is corroded down to a size with the capacity equal to the lock-off load force. Equation 2.7 provides the equation for this computation.

$$
T T F=\left(d_{C}-d_{L O L)}\right) / R
$$

In Equation 2.7, TTF is the time to failure, $d_{C}$ is the current corroded cable diameter as computed in Equation 2.3, $d_{L O L}$ is the diameter with the lock-off load capacity as in Equation 2.6, and $R$ is the user-supplied corrosion rate.

\subsubsection{Corroded wire short axis diameter curve}

The corroded cable diameter that provides the capacity to support the lock-off load force is computed by assigning $C$ in Equation 2.2 to the lockoff load and solving the equation for $d$. Equation 2.2 is a third-order equation, so $d$ is computed using a Newton-Raphson iterative technique. $d_{L O L}$ is assigned the value of $d$. The TTF is then computed using the formula in Equation 2.7 as described above in the "Corroded wire area curve" section.

\subsubsection{Sampling for statistical variability}

Uncertainties exist in the corroded cable pull-test results as shown in Figures 2-1 and 2-2. Uncertainties exist in the lock-off load forces due to factors such as the measurement system when applying tension to the 
anchor cables and relaxation that occurs after tensioning. The nature of the uncertainties must be known so that a distribution for a measurement's variability can be established. The inherent randomness and uncertainty of these model parameters require numerical methods to obtain solutions to the resulting probabilistic problem. A numerical method such as Latin Hypercube simulation is a sampling technique used for conducting the analysis. Latin Hypercube sampling (LHS) was selected for its efficiency and its reduction in run time compared with that of direct Monte Carlo simulation. It has been used in three previous software applications developed by Dr. Ebeling and his team of researchers: GDLAD_Sloping_Base (Ebeling et al. 2008); GDLAD_Foundation (Ebeling et al. 2012); and CPGA-R (Ebeling et al. 2013). When LHS is used in the multivariate case, it is important to maintain statistical independence between variables unless correlation is explicitly specified. LHS performs best when the variable space is orthogonal. The Dakota LHS stand-alone application from Sandia National Laboratories is this ERDC Research and Development teams' software of choice for the LHS of multiple variables. Section 2.4 in Ebeling et al. (2013) discusses the Latin Hypercube simulation methodology in detail.

\subsubsection{Summary of inputs and outputs}

One of the CAS_Stab-R main functions is to provide a probabilistic analysis of RAL for a corroded anchor cable. The inputs required for the RAL function are the following:

- the lock-off load force applied to the anchor cable at installation

- the length of time the anchor cable has been in service

- the method to determine the corroded size of the cable (user-specified corrosion rate, user-specified oxygenation level of the anchor cable environment, or an NDT-measured anchor cable size) and the corresponding corrosion rate

- the distribution information for the variables (lock-off load force and corrosion rate).

CAS_Stab-R provides as output a probabilistic estimate of the RAL computations for the simulation. The RAL estimate is provided in the form of a histogram (an example is shown in Figure 3-82), probability density function (an example is sown in Figure 3-84), cumulative distribution function (an example is shown in Figure 3-86), mean, standard deviation, coefficient of variance, minimum, and maximum value for the RAL samples 
that did not result in a cable failure. The number of samples in the dataset that resulted in cable failures is reported. The vector coordinates for each sample (i.e., the variables listed above) are also presented in histogram form as well.

\subsection{Structural stability against sliding (Probability of Unsatisfactory Performance [PUP] Analysis) computations}

The CAS_Stab-R software implements the ETL 1110-2-256 (HQUSACE 1981) wedge solution method of computing a Factor of Safety (FOS) for a structure against sliding along a defined slip plane. CAS_Stab-R extends the ETL 1110-256 (HQUSACE 1981) wedge solution by allowing structural drains and anchorage forces into the computation.CAS_Stab-R performs a probabilistic analysis of the structure's stability against sliding that provides a PUP measure.

\subsubsection{Wedge solution overview}

ETL 1110-2-256 (HQUSACE 1981) presented a method of determining the FOS for a 2-D model of a hydraulic structure and its foundation. The model is divided into a system of wedges with vertical sides and the potential slip plane at the base of each wedge. The wedges are designated as driving, structural, or resisting. A model can consist of multiple driving or resisting wedges but can have only one structural wedge. The structural wedge begins vertically at the heel of the structure and ends at the toe.

A typical gravity dam free body diagram is shown in Figure 2-10. This diagram consists of a single driving wedge on the upstream side of the dam, the structural wedge, and a single resisting wedge on the downstream side of the dam. The forces on each wedge are analyzed to determine the overall horizontal forces acting on the wedge. A free body diagram of an individual theoretical wedge is shown in Figure 2-11. 
Figure 2-10. Free body diagram of a theoretical structure.

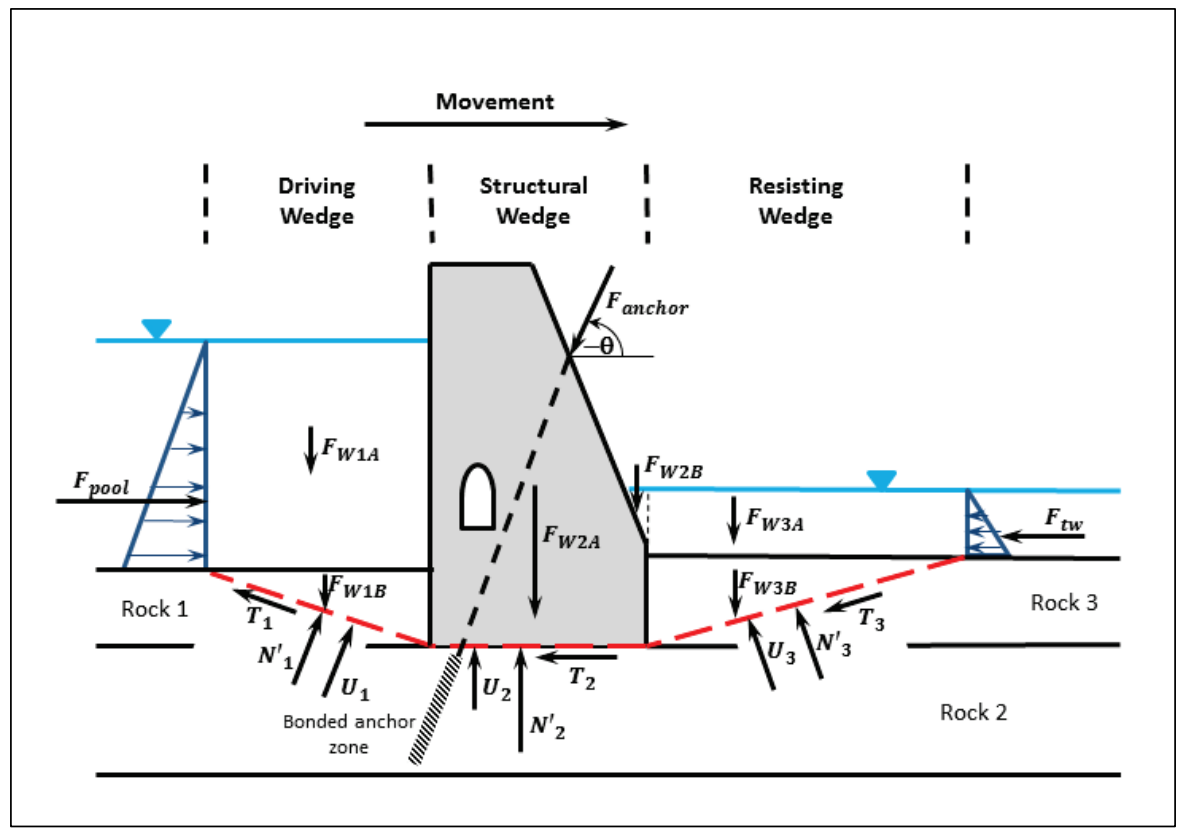

Figure 2-11. Free body diagram of a theoretical wedge.

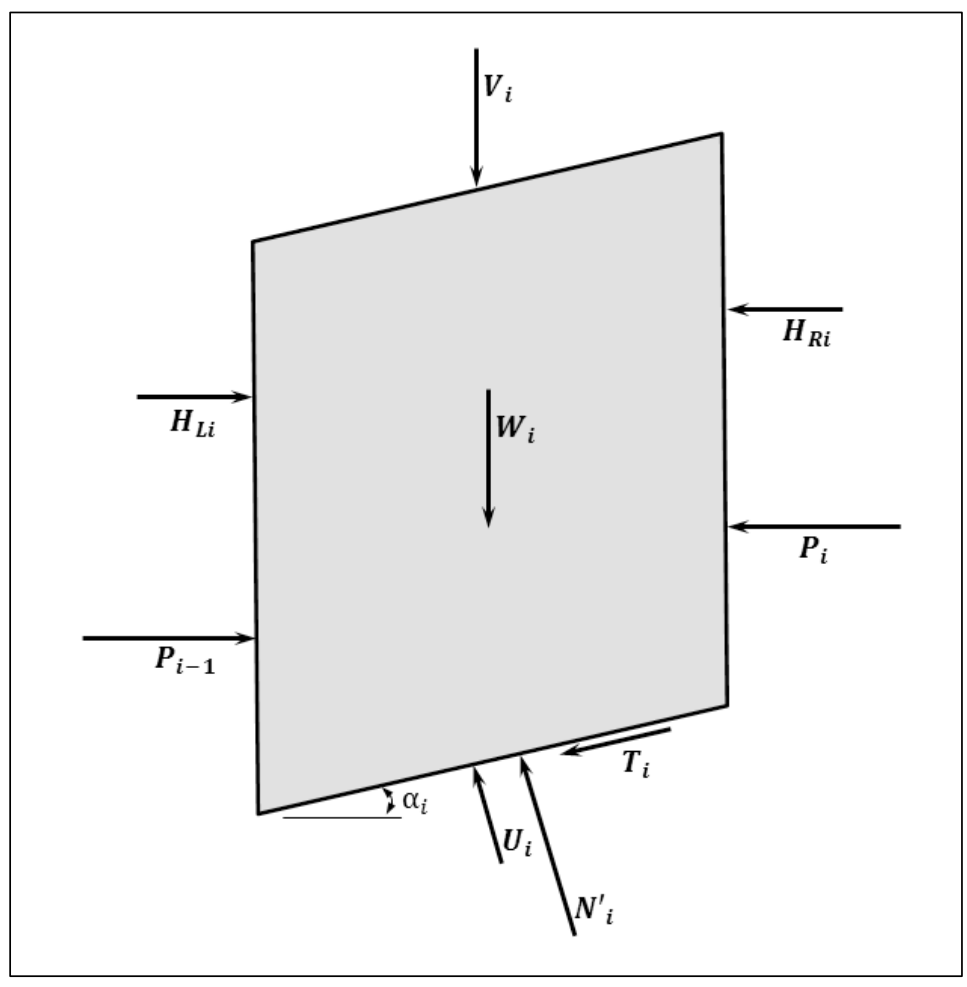


In Figure 2-11, the symbols are as follows:

- $P_{i-1}$ the horizontal interslice force exerted on the $\mathrm{i}^{\text {th }}$ wedge by the $(\mathrm{i}-1)^{\mathrm{th}}$ wedge

- $P_{i}$ the horizontal interslice force exerted on the $(\mathrm{i}+1)^{\mathrm{th}}$ wedge by the $\mathrm{i}^{\text {th }}$ wedge

- $H_{L i}$ horizontal pressure exerted on the $\mathrm{i}^{\text {th }}$ wedge above the top of the (i-1) ${ }^{\text {th }}$ wedge such as pool pressures or silt or soil pressures

- $H_{R i}$ horizontal pressure exerted on the $\mathrm{i}^{\text {th }}$ wedge above the top of the $(\mathrm{i}+1)^{\mathrm{th}}$ wedge such as pool pressures or silt or soil pressures

- $\quad N^{\prime}{ }_{i}$ normal force on the wedge base

- $T_{i}$ shear force on the wedge base

- $U_{i}$ hydraulic uplift force on the wedge base

- $V_{i}$ vertically acting weight or forces other than the wedge weight such as water, silt, soil, anchorage force

- $W_{i}$ weight of the wedge

- $\alpha_{i}$ slope angle of the wedge base from horizontal.

The governing equation derived in ETL 1110-2-256 (HQUSACE 1981) for wedge equilibrium is given in Equation 2.8 .

$$
P_{i-1}-P_{i}=\frac{\left[\left(W_{i}-V_{i}\right) \cos \alpha_{i}-U_{i}+\left(H_{L i}-H_{R i}\right) \sin \alpha_{i}\right] \frac{\tan \varphi_{i}}{F S_{i}}-\left(H_{L i}-H_{R i}\right) \cos \alpha_{i}+\left(W_{i}+V_{i}\right) \sin \alpha_{i}+\frac{c_{i}}{F S_{i}} L_{i}}{\left(\cos \alpha_{i}-\sin \alpha_{i} \frac{\tan \varphi_{i}}{F S_{i}}\right)}
$$

The wedge will be in a horizontal equilibrium state when $P_{i-1}-P_{i}=0$. For multiple wedges, the system will be in horizontal equilibrium when the sum of the horizontal forces acting on all the wedges is equal to 0 . Therefore, the equilibrium Equation 2.8 is computed for each wedge and summed over all of the wedges defined by the user-specified slip plane (Figure 2-10). An iterative process is employed for adjusting the trial FOS value until overall horizontal equilibrium is achieved. In CAS_Stab-R, horizontal equilibrium is considered achieved if the sum of the equilibrium forces is less than or equal to the equilibrium tolerance which defaults to $100 \mathrm{lb}$. If horizontal equilibrium has not been achieved within the designated number of iterations (100 iterations is the default), CAS_Stab-R returns an FOS value of 0.05. For more details of applying the wedge solution to the structural stability analysis, the reader is referred to Chapter 2 of Ebeling et al. (2008). 
Figure 2-12 depicts the free body diagram of a dam with silt and soil boundary pressures acting on the structural wedge. There are no foundation drains in this problem. The vertical forces acting on the driving wedge are the weight of the wedge, $F_{W_{1} B}$, the weight of the pool, $F_{W 1 A}$, and the uplift force, $U_{1}$. The horizontal force acting on the driving wedge is the horizontal interslice force applied by the structural wedge.

Figure 2-12. Dam free body diagram with silt and soil as boundary pressure forces.

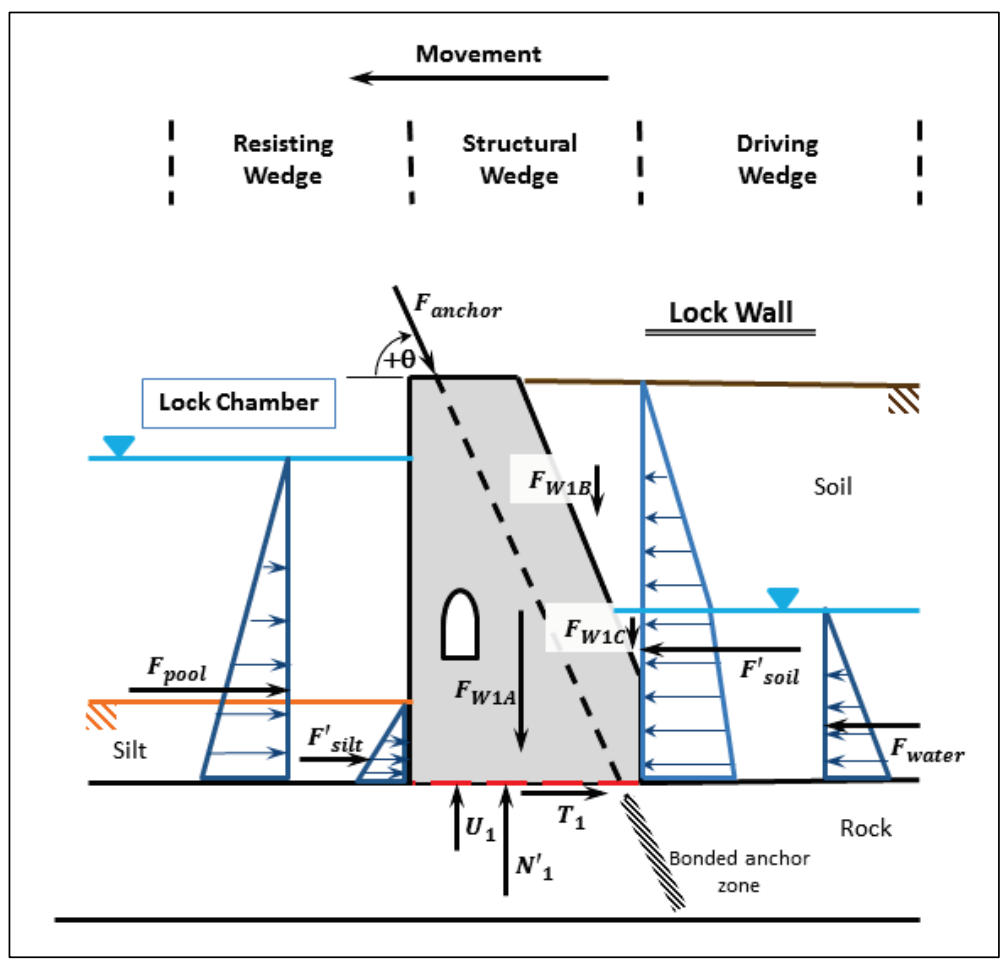

The vertical forces acting on the structural wedge are the weight of the structure, $F_{W_{2} A}$, the weight of the water that lies in the structural wedge, $F_{W_{2} B}$, the vertical component of the anchor force, $F_{\text {anchor }}{ }^{*} \sin (\theta)$, and the uplift force, $U_{2}$. The horizontal forces acting on the structural wedge are the horizontal component of the anchor force, $F_{\text {anchor }}{ }^{*} \cos (\theta)$, the pool water pressure, $F_{p o o l}$, then tailwater water pressure, $F_{t w}$, and the horizontal interslice forces applied by the driving and resisting wedges.

The vertical forces acting on the resisting wedge are the weight of the wedge, $F_{W_{3} B}$, the weight of the pool, $F_{W_{3} A}$, and the uplift force, $U_{3}$. The horizontal force acting on the resisting wedge is the horizontal interslice force applied by the structural wedge. 
The uplift forces, $U_{i}$, are computed as presented in sections 6.3.1 to 6.5.2 of Ebeling et al. (2012). The CAS_Stab-R software implements Flow Options 4,5 , and 6 in the same manner as the GDLAD_Foundation software which is described in the same report.

\subsubsection{Boundary pressure and wedge solution capability for silt and soils}

Normally the forces for silt and soil materials are applied as boundary pressures on the structure as shown in Figure 2-12. The horizontal pressure from the silt is a horizontal distributed force proportional to the at-rest earth pressure coefficient, the density, and the height of the silt. The soil exerts a like horizontal distributed force on the structure. A change in slope of the distribution of horizontal soil pressure with depth occurs at the piezometric water level due to the difference in buoyant density for moist and saturated soil. Effective stresses and hydrostatic pore water pressures are being used to describe the horizontal earth and water forces exerted by the retained soil regime. The resultant force of the pressure distribution exerted by submerged silt below the pool assuming hydrostatic pore water pressures within the silt is given by Equation 2.9.

$$
F_{\text {silt }}^{\prime}=\frac{1}{2} K_{0}\left(\gamma_{\text {saturated }}-\gamma_{\text {water }}\right) H_{\text {silt }}^{2}
$$

The resultant force of the pressure distribution exerted by the soil assuming hydrostatic pore water pressures within the soil is given by Equation 2.10.

$F_{\text {soil }}^{\prime}=K_{0}\left[\frac{1}{2} \gamma_{\text {moist }}\left(H_{\text {soil }}-H_{\text {tailucuter }}\right)^{2}+\gamma_{\text {moist }}\left(H_{\text {soil }}-H_{\text {tailuoter }}\right) H_{\text {tailuater }}+\frac{1}{2}\left(\gamma_{\text {saturated }}-\gamma_{\text {water }}\right) H_{\text {tailuater }}^{2}\right]$

This equation is also used to define $F^{\prime}$ silt for a partially submerged silt layer. The $F^{\prime}{ }_{\text {silt }}$ and $F^{\prime}$ soil contribute a portion of $H_{L i}$ and $H_{R i}$, respectively, in Equation 2.8. $H_{L i}$ and $H_{R i}$ also contain any boundary pressure contributed by the headwater and tailwater.

CAS_Stab-R provides the option for the silt and soil in a model to be applied as boundary pressures to the structural wedge as the default.

Another option is provided for cases where the user-provided slip plane extends into a silt or soil region. An example free body diagram of this type of model is shown in Figure 2-13. In this case, the wedge solution option 
should be chosen. Normal and uplift forces and weight of the silt and soils are applied as for rock regions that lie above the slip plane. In Figure 2-13, the driving wedge values of $U_{1}$ substitutes for $U_{i}, F_{W 1 B}$ for $W_{i}$, and $F_{W 1 A}$ for $V_{i}$ in Equation 2.8. The corresponding substitutions are applied for the resisting wedges.

Figure 2-13. Free body diagram with slip plane in silt and soil.

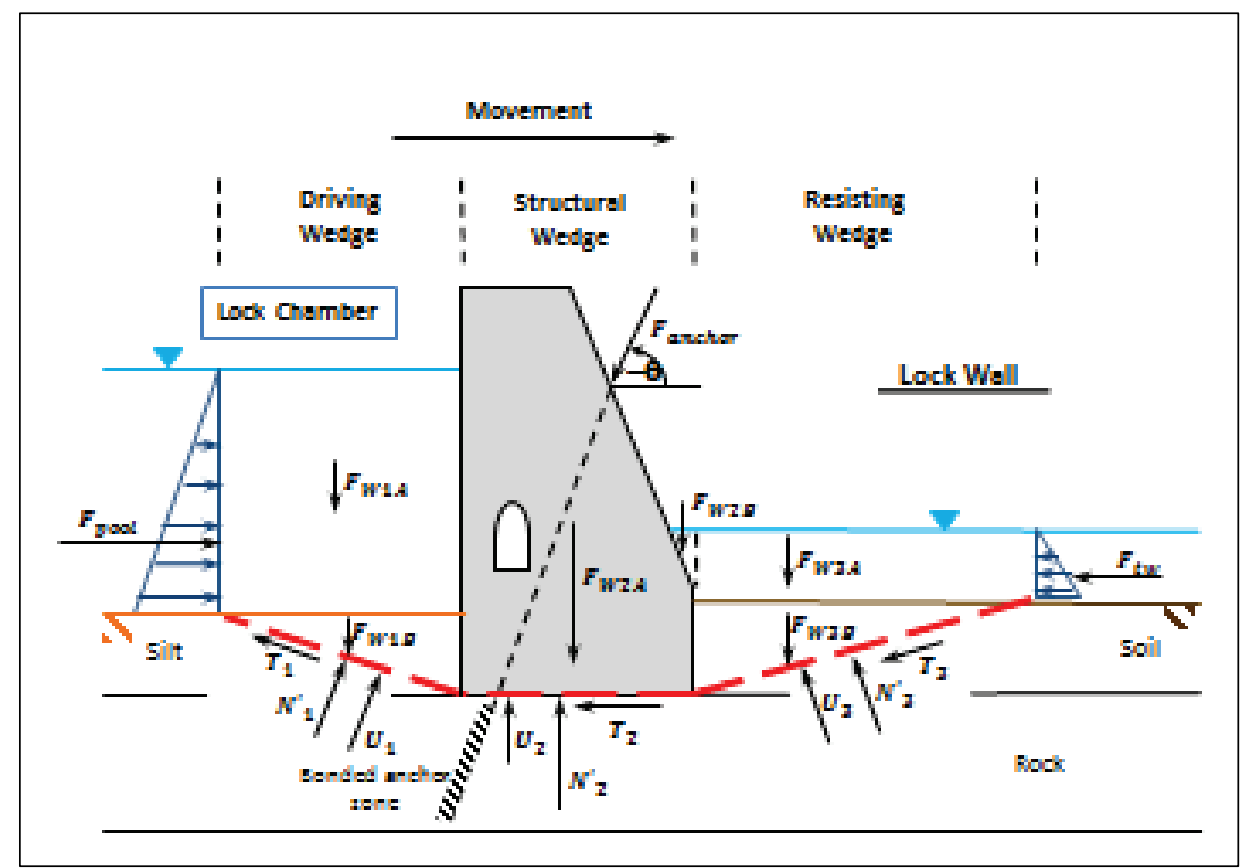

\subsubsection{Reliability methods for calculating the PUP}

The PUP value for a structural system depends on the probabilistic characterization of properties assigned in the stability calculations. Each variable, for each property, is described by a statistical distribution, as discussed in subsection 2.1.2. The statistical distribution is a probability density function that gives the instantaneous probability of any individual value for the variable drawn from the distribution.

The PUP is a value that consists of the integration of probability density function values that in the $G()$ function where any limit state has been exceeded. This calculation is given using the following numerical method, which the authors describe but do not use because it is computationally intractable. In the multivariable space, a $G()$ function can be created relating the resistance $(R)$ to the load $(Q)$ acting for a vector $\boldsymbol{V}$ for every instance of $\boldsymbol{V}$ in the multi-variable space. 


$$
G(\boldsymbol{V})=R(\boldsymbol{V})-Q(\boldsymbol{V})
$$

An indicator function can be created with the express purpose of providing a multiplicative identity for $X$ vector values [i.e., $I(X)=1$ ] where there is unsatisfactory performance [with $R(\boldsymbol{V})<Q(\boldsymbol{V})$ ], and a value of 0.0 at the response surface of the limit state or less [with $R(\boldsymbol{V}) \geq Q(\boldsymbol{V})$ ].

$$
I(\boldsymbol{X})=\left\{\begin{array}{l}
0: G(\boldsymbol{X}) \geq 0.0 \\
1: G(\boldsymbol{X})<0.0
\end{array}\right.
$$

The function to calculate PUP for the Gaussian multivariate space $X$ is

$$
p_{U}=\Phi(\boldsymbol{X})=\int_{\mathbb{R}^{N}} I(\boldsymbol{X}) h(\boldsymbol{X}) d \boldsymbol{X}
$$

where $h(\boldsymbol{X})$ is the probability density function for the space and is given by

$$
h(\boldsymbol{X})=\prod_{n=1}^{N} h\left(X_{n}\right)
$$

Equation 2.13 reveals that the orthogonal probabilities are multiplied to each other, reducing the overall probability of the multivariable instance in the variable space.

The integration in Equation 2.13 can be approximated using a discretization of the space, but these approximations are less accurate with large discrete steps. Using smaller discrete steps improves the accuracy but is numerically intensive, requiring more time to achieve a solution. Therefore, closed form integration of the PUP value is intractable, and another solution method needs to be used.

In order to more accurately approximate the PUP value in a reasonable time, the authors chose to use statistical methods to calculate PUP, namely Monte Carlo simulation techniques. In the Monte Carlo method, a set of randomly chosen samples are generated. Each sample is a vector $(\boldsymbol{X})$ in the multi-variable space with each variable instance being drawn from its probabilistic distribution. Each sample is tested to find the $G(\boldsymbol{X})$ value and receives an indicator value of 1 or $\mathrm{o}$ if it is does or does not exceed the limit state, respectively. Because the samples are randomly spread using the 
distributions for the variables, the approximation takes the distributions directly into account. Of course, more samples imply more accuracy.

The PUP calculation value for a standard Monte Carlo application is given by

$$
P U P=\frac{\sum_{1}^{n} I(\boldsymbol{X})}{n}
$$

The Monte Carlo method can be improved using LHS method. It is described in more detail in section 2.4 of Ebeling et al. (2013). This sampling method requires fewer samples to achieve comparable accuracy to direct Monte Carlo simulation with completely random sample selection. The Latin Hypercube selects samples from a distributed grid in the sample space as depicted in Figure 2-14.

The Monte Carlo method can also be improved using Importance Sampling. Importance Sampling works by transforming the original variable probability density function distributions $[p(x)]$ to $[q(x)]$ distributions with means and standard deviations that are centered near the area of interest (i.e., where the limit state is reached). Samples are taken from the $[q(x)]$ transformed probability density function distributions and the $I(\boldsymbol{X})$ indicator calculations are performed. The resulting value is then untransformed to determine the actual probabilities using the original distributions and Equation 2.16. Figure 2-15 shows the relationship between the original $[p(x)]$ probability density function and the transformed $[q(x)]$ probability density function. This process is described in detail in section 2.6 of Ebeling et al. (2013).

$$
P_{u}=\int f(x) \frac{p(x)}{q(x)} q(x) d x
$$

with $f(x)$ being the performance function. The integral function of Equation 2.16 is represented numerically as Equation 2.17, where $I()$ is the indicator function.

$$
P_{u}=\frac{1}{N} \sum_{i=1}^{N} I\left(G\left(V_{i}\right)\right) \frac{p\left(V_{i}\right)}{q\left(V_{i}\right)} \text { where }\left\{\begin{array}{l}
I=1 \mid G\left(V_{i}\right)<0 \\
I=0 \mid \text { otherwise }
\end{array}\right\}
$$


Figure 2-14. Using the normally distributed regions to assign samples according to the Latin Hypercube method for generating samples.

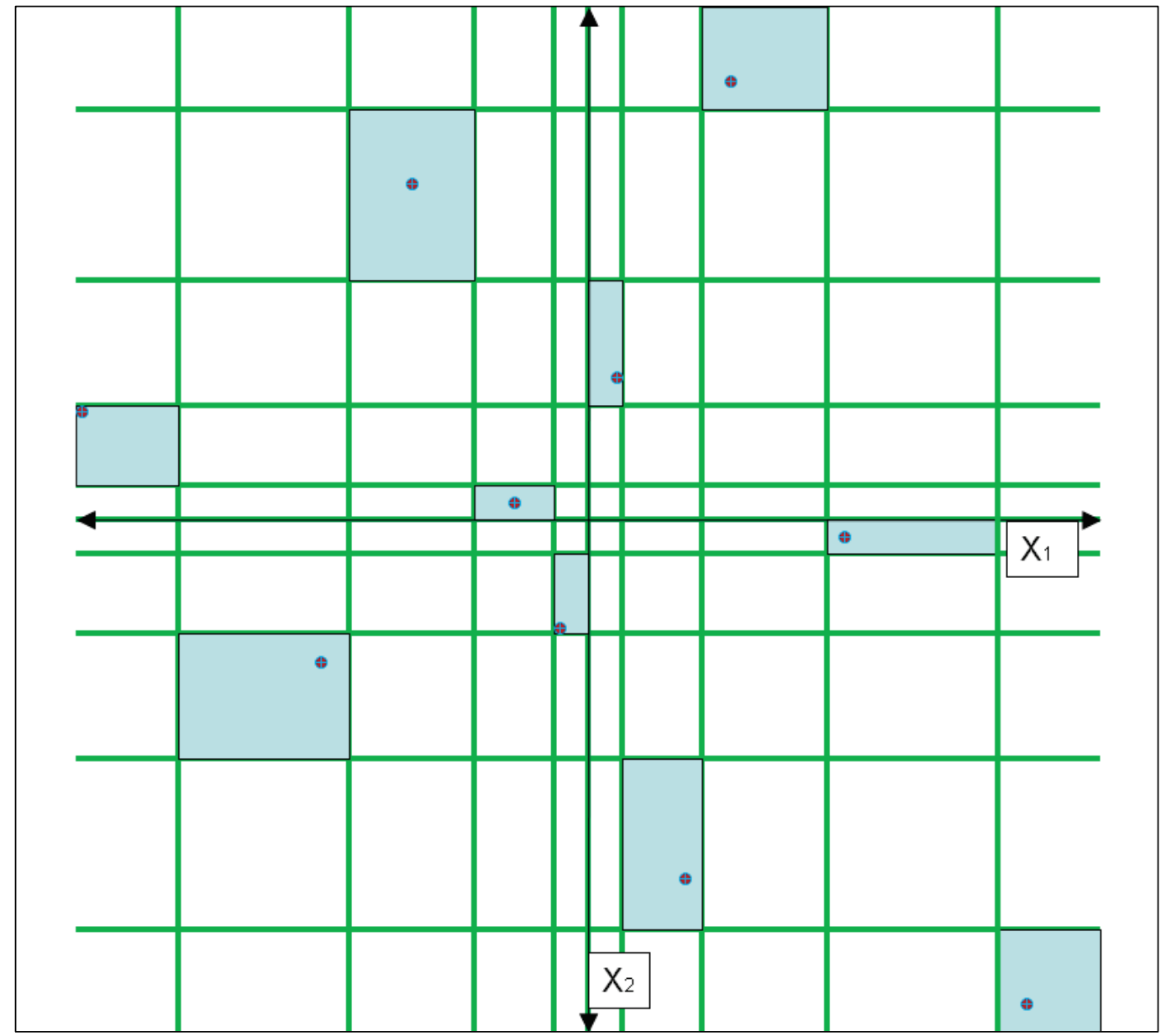

Figure 2-15. For sample $X i$, the distance to the original mean and the distribution mean can be determined and the probabilities computed.

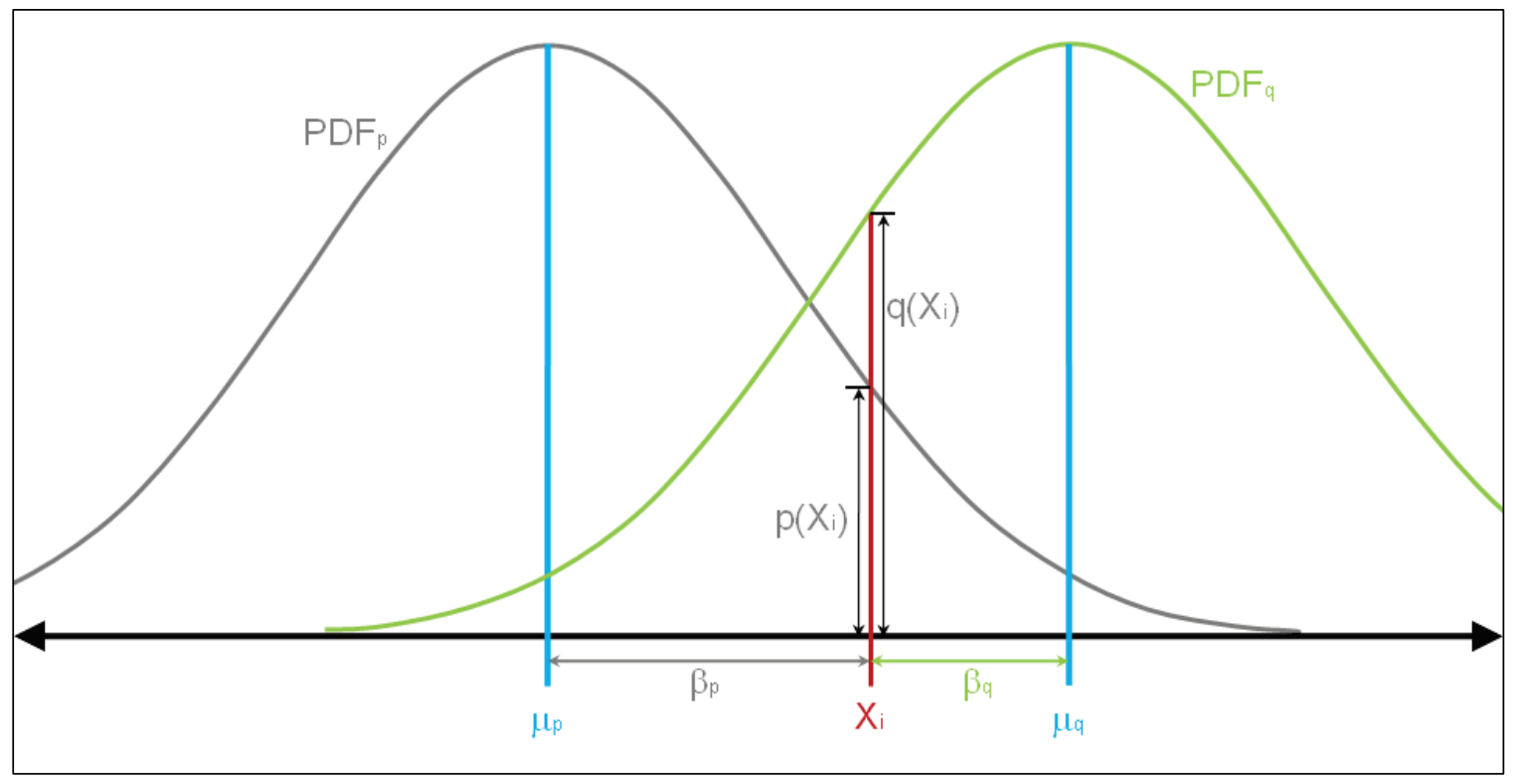


Importance Sampling provides accurate results with fewer samples needed because the samples are chosen in the important region where the limit state is exceeded. This method can be combined with the LHS selection in the transformed distribution space to allow for even fewer samples to provide sufficient accuracy.

In order to find the central location to be used for Importance Sampling, a method for locating a point (also known as the design point) on the limit state response surface needs to be determined. The ASM method is used to determine the design point that lies on the limit state response surface. The location of this design point is defined relative to the mean variable values and is the closest point to the response surface from the means. Because this point lies at the closest point on the response surface, it is the best point to center the Importance Sampling transformed distributions. The ASM algorithm is described in subsection 2.2.5.1.

The most efficient method to determine the PUP value relies on using (1) the ASM method in conjunction with (2) the simulation method using Importance Sampling with LHS.

The ASM method uses the following three equations, which describe the hyperplanar limit state response surface, to determine a design point to center the Importance sampling region. The ASM technique works because any hyperplanar surface can be fully described by a directional cosine vector $(\alpha)$ and the distance from the origin $(\beta)$ for the closest point on the hyperplanar surface (Figure 2.12 in Ebeling et al. [2013]). This resultant directional cosine vector $(\alpha)$ is perpendicular to the hyperplanar surface labeled in Figure 2.12 of Ebeling et al. (2013). The hyperplane is the resultant ASM Response Surface [i.e., where G() = o]. Note that this resultant directional cosine vector $\alpha$ is a unit vector, with the magnitude of each component vector along a variable $\mathrm{X}_{\mathrm{i}}$ axis, $\left|\alpha_{i}\right|$, being a cosine value for that direction with respect to the unit vector $\alpha$. The components of the directional cosine vector $\alpha_{i}$ multiplied by the distance $\beta$ give the distance along each respective variable axes to the design point. In general terms and for the $v$ space, the vector $\boldsymbol{V}$ s components (that define the hyperplane) are given by

$$
V_{i}=\mu_{v_{i}}-\alpha_{i} \beta \sigma_{v_{i}}
$$

The response surface for vector $\boldsymbol{V}$ is defined by 


$$
G(\mathbf{v})=0.0
$$

This can be performed by setting

$$
\alpha_{i}=\frac{\left(\frac{\partial G()}{\partial V_{i}}\right) \sigma_{V_{i}}}{\sqrt{\left[\sum_{i=1}^{n}\left(\frac{\partial G()}{\partial V_{i}}\right)^{2} \sigma_{V_{i}}^{2}\right]}}
$$

with the initial trial value for $\beta$ set to o.o, so that the initial vector $\boldsymbol{V}$ is composed of only the mean values. A numerical procedure is used in conjunction with Equations 2.18, 2.19, and 2.20 to calculate the $\alpha_{i}$ values for each of the $i$ variables.

\subsubsection{Material variables}

The geotechnical parameters that characterize the foundation materials and configuration are uncertain in nature. This can be due to lack of homogeneity in the geologic or man-made material regions, lack of adequate knowledge of the material properties, incomplete or imprecise laboratory test data, etc. The uncertainties that affect the anchorage cables have been discussed in section 2.1.2 above. The CAS_Stab-R software provides for variability in the following parameters that affect the stability analysis:

- effective cohesion for silt, soils and rock materials

- effective internal friction angle for the rock materials and silt and soils if wedge solution option is chosen

- horizontal pressure coefficient for silt and soils

- anchorage lock-off load force

- corrosion rate variable for the selected corrosion rate method.

For the probabilistic stability analysis, the variables are specified as normal and non-normal distributions of values.

\subsubsection{Dealing with non-normal distributions}

An ASM design point solution must be performed in an uncorrelated normalized normal distribution space as described in Chapter 2 of Ebeling et al. (2013). For each normal variable listed, a mean value and either 
standard deviation or coefficient of variation (COV) must be specified to represent the distribution of values. Variables with non-normal distributions (e.g., log-normal, truncated normal, truncated log-normal, uniform, and triangle distributions) must be transformed to a representative normal distribution for use by the ASM reliability method.

For the ASM reliability method to return a value of probability based on $\beta$, all the random variables must be independent and distributed according to a normal distribution, according to the Hasofer-Lind approach (Baecher and Christian 2003; Hasofer and Lind 1974). To handle non-normal distributions, the non-normal distribution variable must be changed to an equivalent normally distributed random variable. This is called the Rosenblatt transformation (Rosenblatt 1952). Rackwitz and Fiessler (1976, 1978) impose two conditions on the equivalent normally distributed random variable that enable the determination of the two parameters of that distribution, $\mu_{V_{i}}^{N}$ and $\sigma_{V}^{N}$. The cumulative distribution functions (CDF) and probability density function of the non-normal and normal distributions should be equivalent at the current design point for the performance function.

These conditions (CDF and probability density function) can be expressed, respectively, in

$$
\begin{gathered}
\Phi\left(\frac{V_{i}-\mu_{V_{i}}^{N}}{\sigma_{V_{i}}^{N}}\right)=F_{i}\left(V_{i}\right) \\
\phi\left(\frac{V_{i}-\mu_{V_{i}}^{N}}{\sigma_{V_{i}}^{N}}\right)=f_{i}\left(V_{i}\right)
\end{gathered}
$$

where $F_{i}$ is the non-normal $\mathrm{CDF}, f_{i}$ is the non-normal probability density function, $\Phi$ is the standard normal CDF, and $\phi$ is the standard normal probability density function. From these conditions, it can be shown that the standard deviation and mean respectively, are the following:

$$
\begin{gathered}
\sigma_{V_{i}}^{N}=\frac{\phi\left(\Phi^{-1}\left[\mathrm{~F}_{\mathrm{i}}\left(\mathrm{V}_{\mathrm{i}}\right)\right]\right)}{f_{i}\left(V_{i}\right)} \\
\mu_{V_{i}}^{N}=V_{i}-\Phi^{-1}\left[F_{i}\left(V_{i}\right)\right] \sigma_{V_{i}}^{N}
\end{gathered}
$$


The probability density function of a standard normal distribution is a continuously positive function. Bounded distributions including triangular probability density functions are not. While it is possible to get equivalent CDF values between the standard normal distribution and a bounded nonnormal distribution (Figure 2-16), it might be impossible to find an equivalent probability density function between distributions. In this case, the standard deviation can be calculated and the mean of the standard deviation varied to find the appropriate $\mathrm{CDF}$ value. The probability density function in this case will be a close estimate at convergence.

Figure 2-16. Finding the position of equivalent cumulative probability for a normal distribution as from a non-normal distribution (after Ang and Tang 1984).

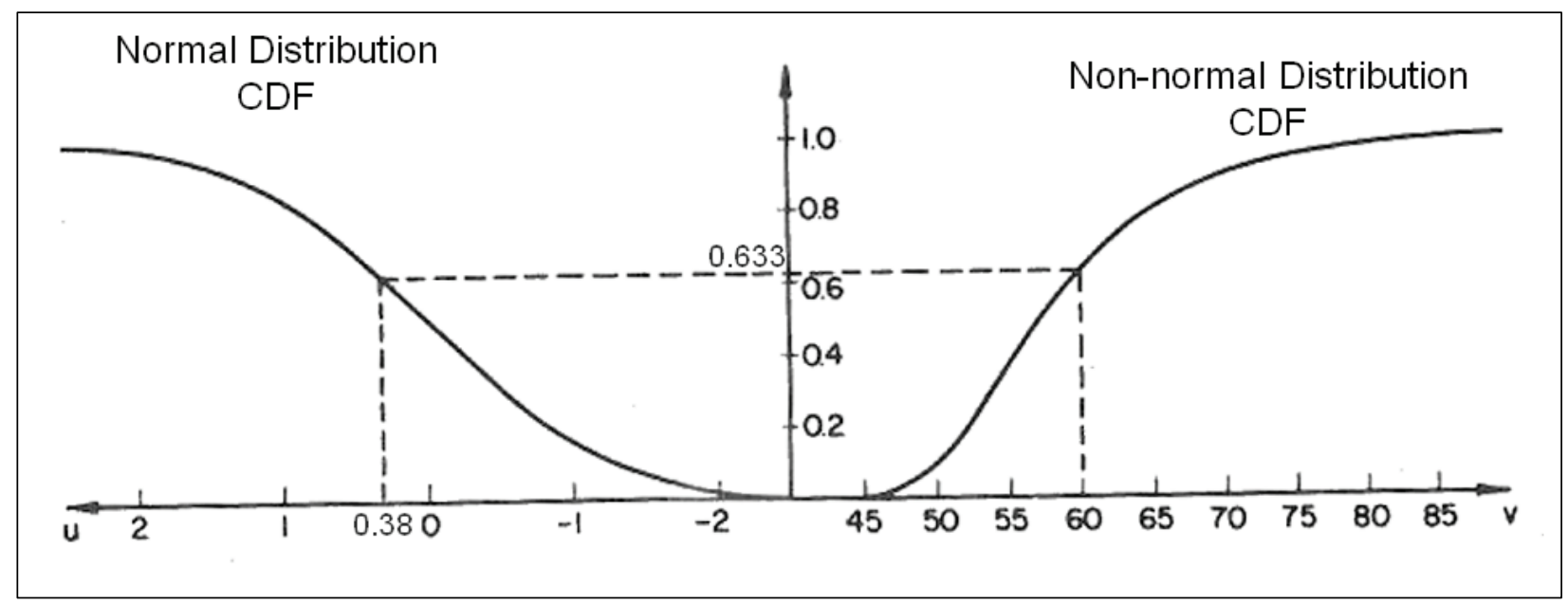

\subsubsection{Dealing with correlated variables}

In certain situations, it is unrealistic to believe that variables chosen for a reliability analysis will be independent of one another. In these situations, variables may be related to some degree such as the case for the soil material properties of the angle of internal friction and cohesion for soil layers. For this discussion, a pair of variables $\left(V_{1}\right.$ and $\left.V_{2}\right)$ is correlated with a correlation coefficient $(\rho)$. If the two variables have a standard normal distribution, they can be changed to a pair of non-correlated variables by solving for two eigenvalues and the corresponding eigenvectors as follows:

$$
\begin{aligned}
& V_{1}^{\prime}=\frac{1}{2 t}\left(\frac{V_{1}-\mu_{V_{1}}}{\sigma_{V_{1}}}+\frac{V_{2}-\mu_{V_{2}}}{\sigma_{V_{2}}}\right) \\
& V_{2}^{\prime}=\frac{1}{2 t}\left(\frac{V_{1}-\mu_{V_{1}}}{\sigma_{V_{1}}}-\frac{V_{2}-\mu_{V_{2}}}{\sigma_{V_{2}}}\right)
\end{aligned}
$$


where $t=\sqrt{0.5}$. The variances for these two variables are equal to the eigenvalues $(\lambda)$ as follows:

$$
\begin{aligned}
& \sigma_{V_{1}^{\prime}}^{2}=\lambda_{1}=1+\rho \\
& \sigma_{V_{2}^{\prime}}^{2}=\lambda_{2}=1-\rho
\end{aligned}
$$

The directional cosines for these newly uncorrelated values must be determined from the original correlated values using the following:

$$
\begin{aligned}
& \alpha_{V_{1}^{\prime}}=\frac{\left[\left(\frac{\partial G()}{\partial V_{1}}\right) t \sigma_{V_{1}}+\left(\frac{\partial G()}{\partial V_{2}}\right) t \sigma_{V_{2}} \sqrt{1+\rho}\right]}{\left[\left(\frac{\partial G()}{\partial V_{1}}\right)^{2} \sigma_{V_{1}}^{2}+\left(\frac{\partial G()}{\partial V_{2}}\right)^{2} \sigma_{V_{2}}^{2}+2 \rho\left(\frac{\partial G()}{\partial V_{1}}\right)\left(\frac{\partial G()}{\partial V_{2}}\right) \sigma_{V_{1}} \sigma_{V_{2}}\right]^{1 / 2}} \\
& \alpha_{V_{2}^{\prime}}=\frac{\left[\left(\frac{\partial G()}{\partial V_{1}}\right) t \sigma_{V_{1}}-\left(\frac{\partial G()}{\partial V_{2}}\right) t \sigma_{V_{2}} \sqrt{1-\rho}\right]}{\left[\left(\frac{\partial G()}{\partial V_{1}}\right)^{2} \sigma_{V_{1}}^{2}+\left(\frac{\partial G()}{\partial V_{2}}\right)^{2} \sigma_{V_{2}}^{2}+2 \rho\left(\frac{\partial G()}{\partial V_{1}}\right)\left(\frac{\partial G()}{\partial V_{2}}\right) \sigma_{V_{1}} \sigma_{V_{2}}\right]^{1 / 2}}
\end{aligned}
$$

and

$$
\begin{aligned}
& V_{1}=\mu_{V_{1}}-\sigma_{V_{1}} t \beta\left(\alpha_{V_{1}^{\prime}} \sqrt{\lambda_{1}}+\alpha_{V_{2}^{\prime}} \sqrt{\lambda_{2}}\right) \\
& V_{2}=\mu_{V_{2}}-\sigma_{V_{2}} t \beta\left(\alpha_{V_{1}^{\prime}} \sqrt{\lambda_{1}}-\alpha_{V_{2}^{\prime}} \sqrt{\lambda_{2}}\right)
\end{aligned}
$$

Ditlevsen (1981) presents a detailed derivation of these equations for transforming correlated random variables into corresponding noncorrelated random values.

\subsubsection{Deterministic solution of Factor of Safety (FOS)}

The CAS_Stab-R software provides the option of executing the wedge solution to obtain the FOS for the model in a deterministic fashion. When this option is chosen, a single wedge solution computation is performed using only the mean values of the variables to obtain the FOS. 


\subsubsection{Advanced First Order Second Moment (ASM) design point determination and importance sampling}

A system's reliability is based on its performance as the values in the variable space vary probabilistically. A limit state is the boundary between satisfactory and unsatisfactory performance of the system, such as an FOS against sliding value of 1.0. An FOS below 1.0 indicates unsatisfactory performance. An FOS above 1.0 indicates satisfactory performance. The limit state boundary is represented mathematically by a performance function that is negative for unsatisfactory performance, positive for satisfactory performance, and 0.0 on the limit state boundary. The performance function can be defined with respect to the capacity of the system, $R$, and the demand on the system, $Q$. With respect to the capacity and demand, the performance function becomes

$$
G(R, Q)=R-Q
$$

This process is discussed in detail in section 2.2 of Ebeling et al. (2013).

\subsubsection{The ASM algorithm}

The ASM algorithm presented in this subsection has been modified from the AFOSM method to include the provision of working with non-normal distributions and correlated random variables.

1. Assign the mean value for each random variable as a starting design point value (i.e., $\left.\left(V_{1}, V_{2}, \ldots, V_{N}\right)=\left(\mu_{1}, \mu_{2}, \ldots, \mu_{N}\right)\right)$.

2. Compute the standard deviation and mean of the equivalent normal distribution for each non-normal random variable by using Equations 2.23 and 2.24 or by adjusting the mean so that an equivalent CDF is determined for the standard deviation of the original data.

3. Compute the partial derivative, $\partial G O / \partial V_{i}$, of the performance function with respect to each non-correlated random variable evaluated at the design point as needed to satisfy Equation 2.20.

4. Compute the directional cosine, $\alpha_{i}$, for each non-correlated random variable as given in Equation 2.20 at the design point. For correlated pairs of random variables, Equations 2.27a and b should be used.

5. Compute the reliability index, $\beta$, by substituting Equation 2.20 for noncorrelated random variables and Equations 2.27a and b for correlated random variables into the $G($ ) performance function of the vector $\boldsymbol{V}$ 
(Equation 2.18) and satisfying the limit state $G()=O$ (Equation 2.19) in using a numerical root-finding method.

6. Compute a new estimate of the design point by substituting the resulting reliability index, $\beta$, obtained in Step 5 into Equation 2.18 for noncorrelated random variables and Equations 2.28a and $\mathrm{b}$ for correlated random variables.

Repeat Steps 2 through 6 until the reliability index, $\beta$, converges within an acceptable tolerance, $\delta$.

\subsubsection{ASM design point determination}

CAS_Stab-R uses the ASM technique to find the minimum distance that a capacity-vs.-demand surface, based on the performance function G(), is from the origin of a defined vector space. This provides the design point to be used as the center of the Importance Sampling sample space. The coordinates of the point found by the ASM method are then used as the mean values for the Importance Sampling variable distributions for sample space generation, which will be back projected to the original distribution space. This process is discussed in detail in section 2.5 of Ebeling et al. (2013).

\subsubsection{Importance sampling}

CAS_Stab-R uses this function as the basis of determining a point on the limit state surface (where $G(R, Q)=0.0$ ) about which the vectors that make up the sample space will be centered. Centering the sample space for a simulation around a point that lies on the limit state is referred to as Importance Sampling as the samples generated will be centered on the limit state boundary rather than in an area where samples are unlikely to be drawn that meet or exceed the limit state. The sample's probabilities are then renormalized to the original distribution space so that a more accurate PUP value calculation can be performed. This process is discussed in detail in section 2.6 of Ebeling et al. (2013).

\subsubsection{PUP calculation}

The limit state for the FOS is 1.0 for the $G(\boldsymbol{V})$ function. When the FOS is less than 1.0, the structural model has performed unsatisfactorily and $G(\boldsymbol{V})$ returns a value less than 0 . When the FOS is greater than or equal to 1.0, performance is satisfactory, and $G(\boldsymbol{V})$ returns a value greater than or 
equal to o. Recall that $G(\boldsymbol{V})$ is the capacity minus the demand and that FOS is the ratio of the capacity with respect to the demand.

Dakota software is used to generate the vectors for the Latin Hypercube sample space centered about the ASM-determined design point. Dakota software is used for the reasons explained in section 2.1.4 above. For each sample in the sample space, the $G(\boldsymbol{V})$ function is executed, and the result is stored. The PUP value is calculated using the Importance Sampling equation.

$$
P_{u}=\frac{1}{N} \sum_{i=1}^{N} I\left(G\left(\boldsymbol{V}_{i}\right)\right) \frac{p\left(V_{i}\right)}{q\left(V_{i}\right)} \text { where }\left\{\begin{array}{l}
I=1 \mid G\left(\boldsymbol{V}_{i}\right)<0 \\
I=0 \mid \text { otherwise }
\end{array}\right\}
$$

\subsubsection{Summary of inputs and outputs}

The inputs required to perform a PUP analysis for sliding of a stranded cable anchored navigation structure utilizing recently published results of corroded stranded cable pull tests are the following:

- structure geometry and geotechnical properties(unit weight)

- foundation materials geometry and geotechnical properties (unit weight, cohesion, internal friction angle, horizontal pressure coefficient)

- fill materials (silt and or soils) geometry and geotechnical properties (moist unit weight, saturated unit weight, horizontal earth pressure coefficient)

- pool levels

- tailwater levels

- plane on which slippage is expected

- method of corrosion prediction (user-specified corrosion rate, oxygenation level in the anchor cable environment, or NDTdetermined anchor cable size)

- value of corrosion rate (if the user-defined corrosion rate is known)

- value of oxygenation level (if oxygenation level is known)

- value of NDT-determined anchor cable size (if known)

- length of time the anchor cable has been in service

- the number of samples for the sample space

- equilibrium force tolerance

- maximum number of iterations for FOS determination

- simplified seepage model choice. 
The CAS_Stab-R software provides two outputs for the structural stability against sliding function. One output is the FOS for the model obtained from a deterministic calculation. The other output is the PUP value determined by a probabilistic analysis. 


\section{CAS_Stab-R Visual Modeler Software}

\subsection{Introduction}

This chapter discusses the Visual Modeler for CAS_Stab-R software. The Visual Modeler allows the user to create the input data that will be processed to produce analysis results that can be visualized to provide meaningful information. Example problems will be used to demonstrate the features for input and output visualization to demonstrate the program operation.

\subsection{CAS_Stab-R functions}

CAS_Stab-R provides the user with two primary functions. The first function is the Probabilistic Stability Analysis of a Structure. This function provides the user methods to describe a cross section of a hydraulic structure, its foundation elements, and surrounding topography. This description is used in a structural analysis to determine the PUP. The reduced capacity of the anchorage is modeled using statistical equations from recently published results of pull tests on corroded seven-strand cables (Ebeling et al. 2016).

The second function is the RAL probabilistic estimate for a particular cable. This estimate is modeled using reduced capacities based upon the pull-test data in the prior paragraph reference.

\subsection{CAS_Stab-R main screen}

CAS_Stab-R was developed to execute on Windows-based computer systems. Upon startup, the window shown in Figure 3-1 is displayed. The user can enter a Project Name at any time for the analysis work to be performed. The two options available, (1) Analysis of Hydraulic Structure Stability Against Sliding and (2) Lifetime of Anchor (LoA), are discussed in the following sections 3.4 and 3.5 , respectively. 
Figure 3-1. CAS_Stab-R main screen display.

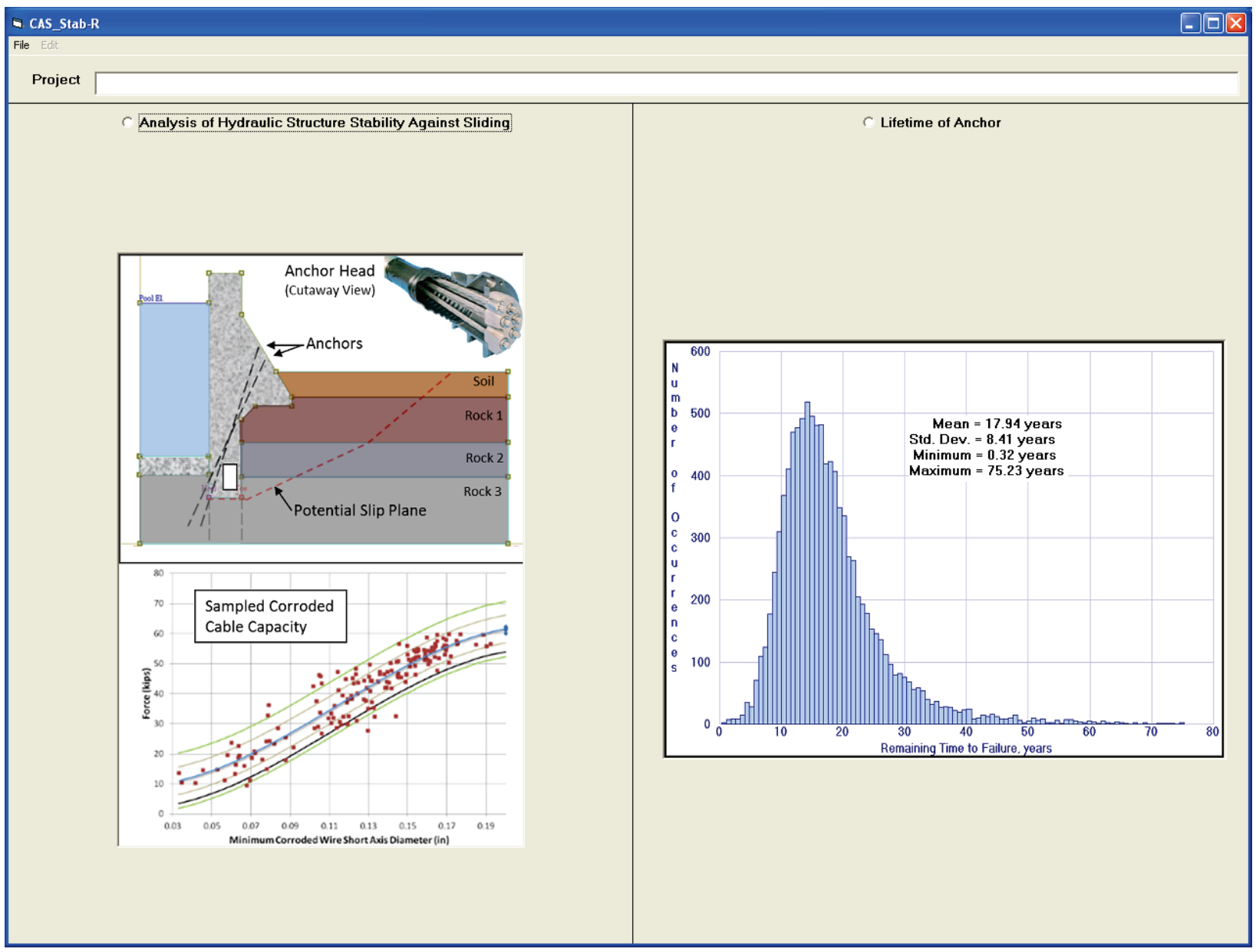

\subsection{Analysis of hydraulic structure stability against sliding}

Choosing this option provides a means of entering model information for the cross section of a navigation structure (such as a dam or lock wall) and its surrounding topology. This input is then analyzed to generate a PUP against sliding. The input needed to perform an analysis of this type is the following:

- structure geometry (includes the gallery, drain, and anchorage information)

- material regions geometry and composition

- silt level and composition

- soil levels and composition

- headwater, tailwater, and/or piezometric levels

- potential slip plane location

- flow condition. 
Choosing the Analysis of Hydraulic Structure Stability Against Sliding option button on the window shown in Figure 3-1 loads the tabbed input window shown in Figure 3-2. The various components of the Figure 3-2 window are discussed in the following subsections.

Figure 3-2. Structural stability Introduction tab.

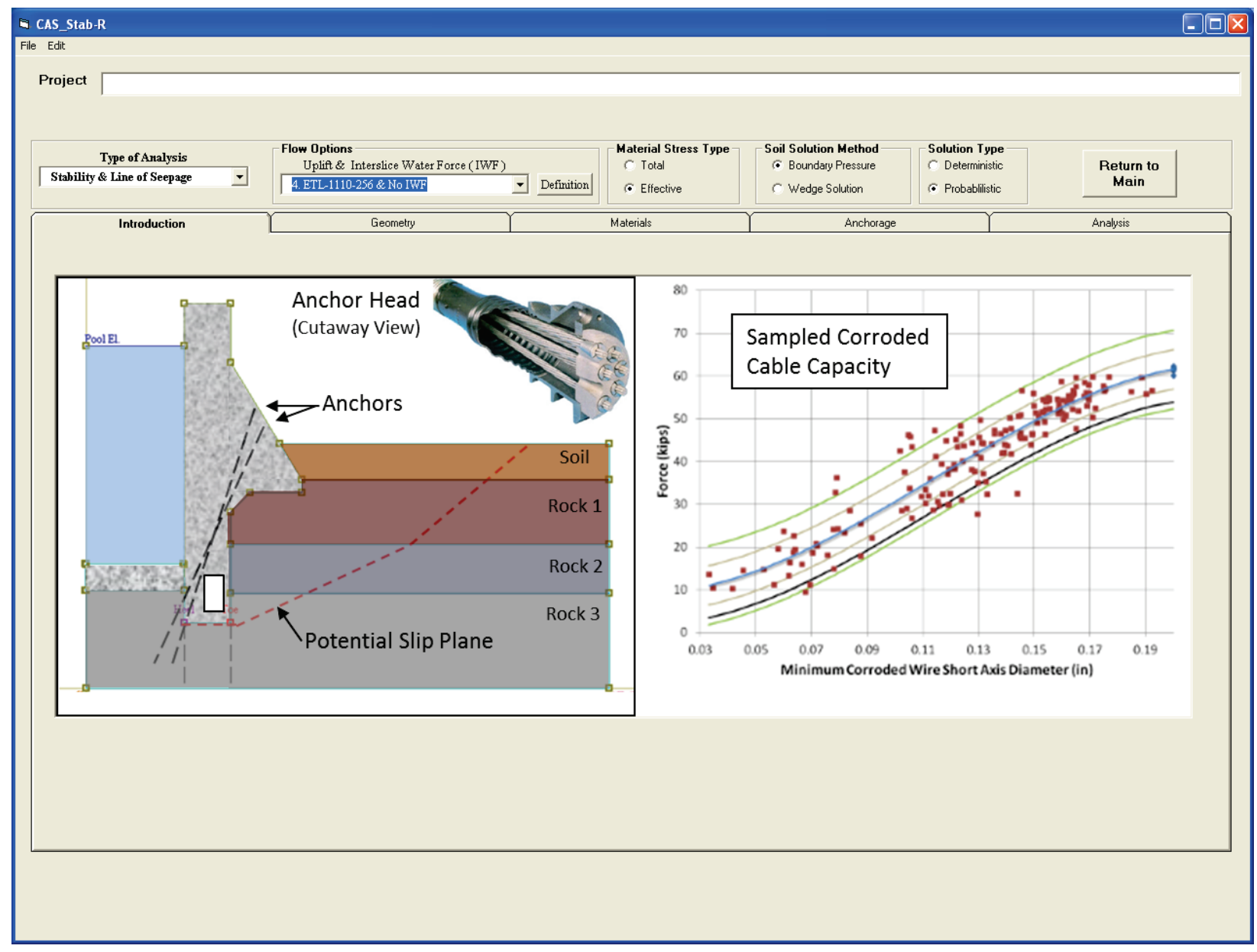

\subsubsection{File menu}

The Analysis of Hydraulic Structure Stability Against Sliding window has File menu options as shown in Figure 3-3. The File menu provides options to initialize a new model (New), to load a model configuration from an existing file (Open), to save the current model configuration to a file (Save and Save As), to print the current model geometry (Geometry-Print Geometry) and to exit the program (Exit). 
Figure 3-3. Structural stability File menu.

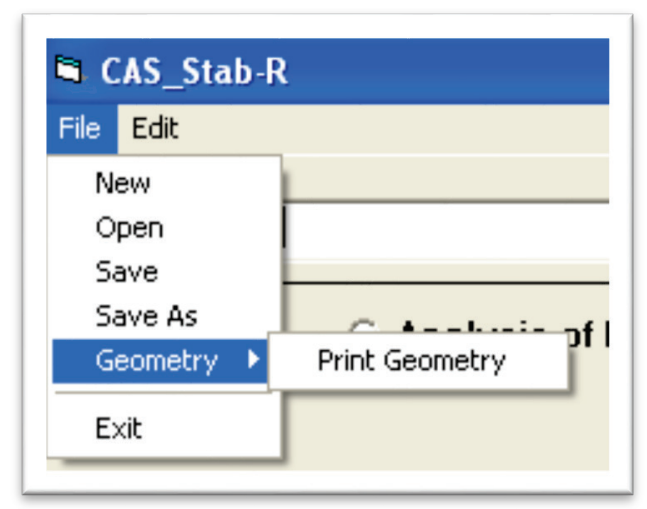

\subsubsection{File - New}

Choose the File - New option to reinitialize the program to the startup state. This option will remove the currently active model states from memory.

\subsubsection{File - Open}

Choose the File - Open option to load a previously saved model. Clicking the Open option will load an Open File dialog box as seen in Figure 3-4.

Select the file that holds the model information to read and click the Open button. During the file-read operation, message boxes may be generated to alert the user to expected information missing from the file. The cause of these messages is most likely due to a file saved during a partially completed model. Upon completion of the file-read operation, the Geometry tab will be activated. The Geometry tab is shown in Figure 3-14. Figure 3-16 shows the Geometry tab with a sample model geometry.

The model configuration files are stored in a text file, and parameters are indicated using a set of keywords. The keywords are documented in Appendix A of this report. A keyword file can be generated by use of the CAS_Stab-R File - Save (As) functions or by use of an ASCII text editor. 
Figure 3-4. File Open dialog box.

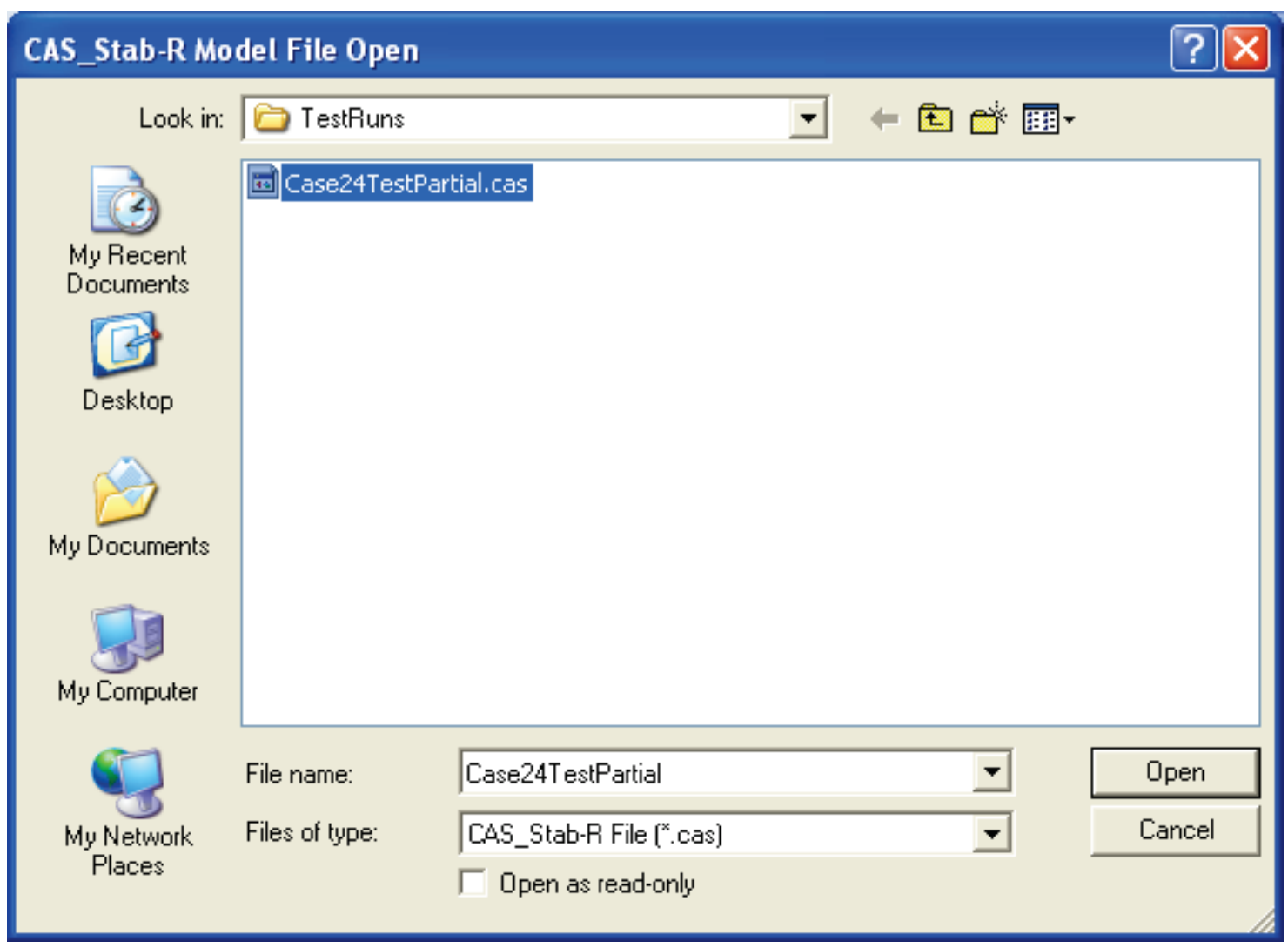

\subsubsection{File - Save}

Choose the File - Save option to write the current model configuration to the currently selected CAS_Stab-R model file. If a file has not been previously opened, then a Save As operation is performed as described in the following sub-section 3.4.1.4.

\subsubsection{File - Save As}

Choose the File - Save As option to save the current model configuration to a new file name. Clicking this option produces a File Save As dialog box as shown in Figure 3-5.

Specify the name of the file to store the current model configuration and click the Save button. 
Figure 3-5. File Save As menu option.

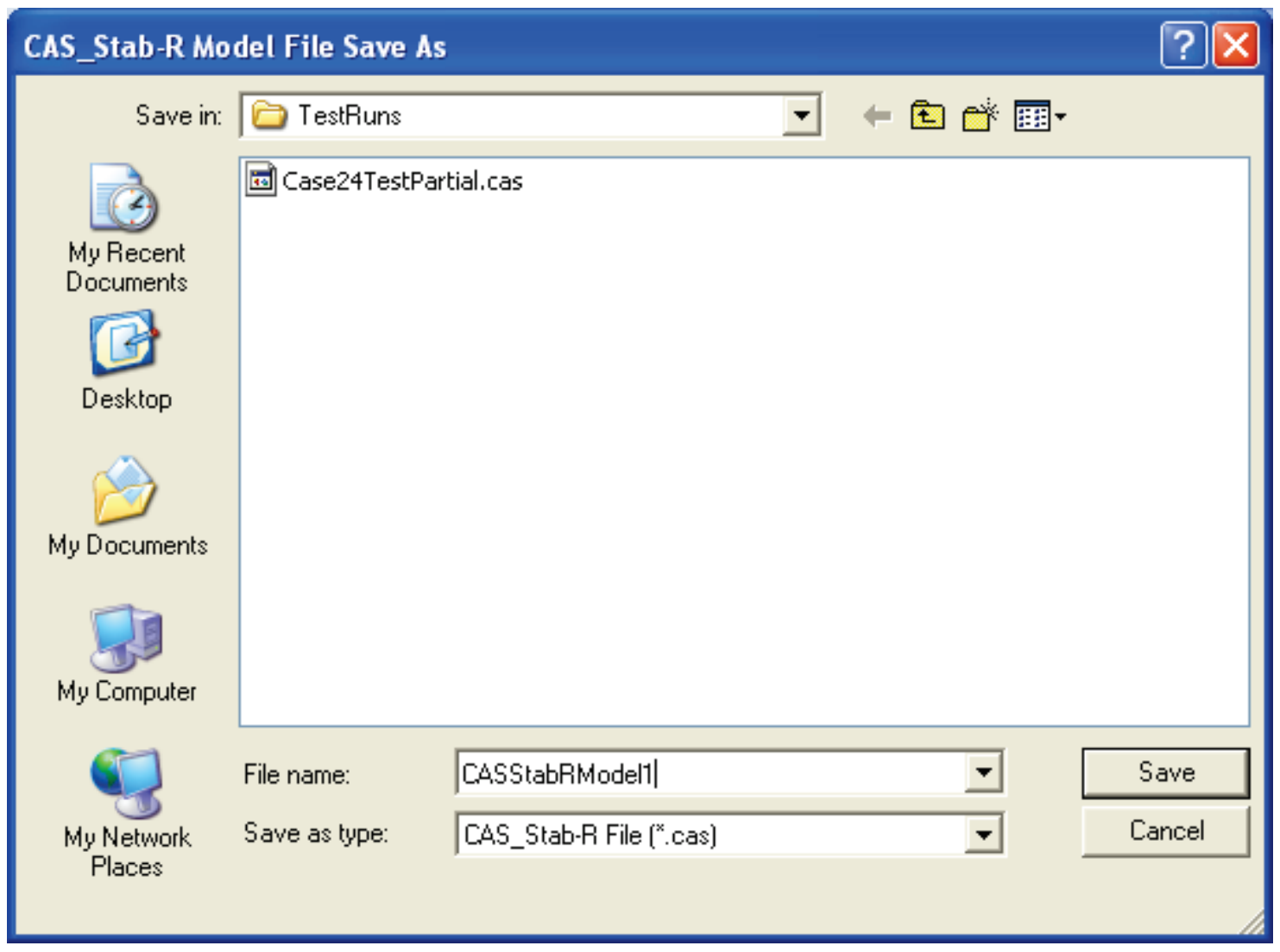

\subsubsection{File - Geometry - Print Geometry}

The current geometry as seen in the Geometry Display of the Geometry tab can be printed by selecting the Geometry - Print option from the File menu as shown in Figure 3-6.

Figure 3-6. File-Geometry-Print Geometry menu option.

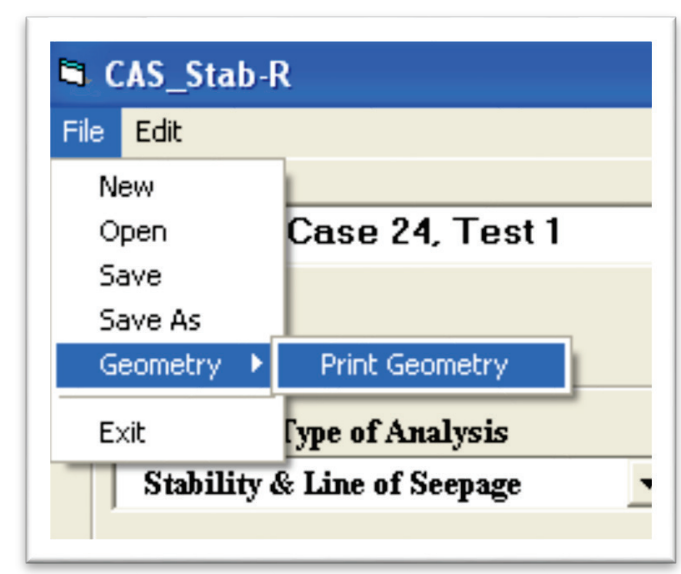


After the Print Geometry option is clicked, a Print dialog box is will appear as shown in Figure 3-7. Select the desired printer, set the appropriate printer options, and click the Print button.

Figure 3-7. Print dialog box.

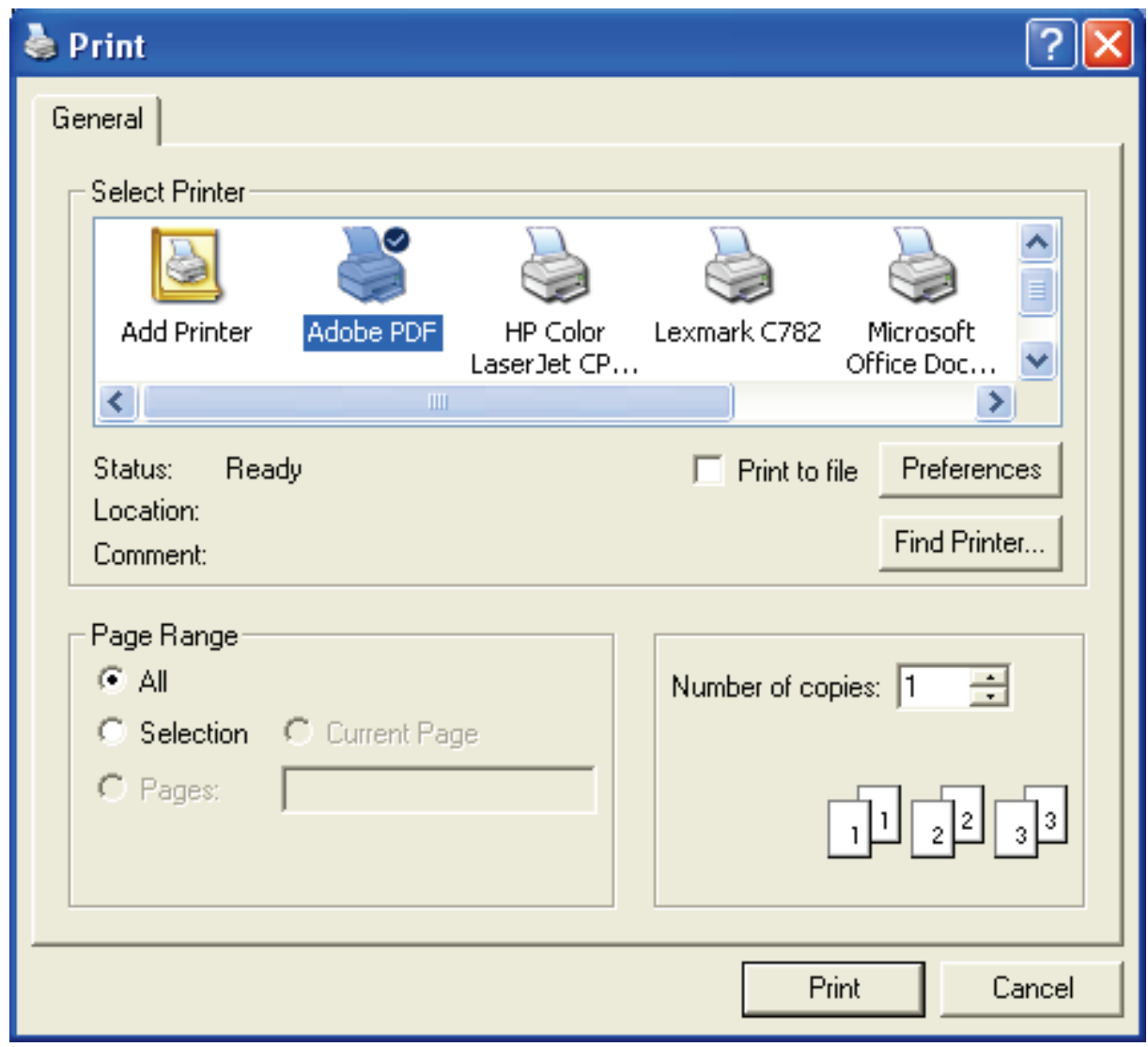

\subsubsection{File - Exit}

Choose the File - Exit option to close the CAS_Stab-R program. Any model configuration changes that have not been saved will be lost. Therefore, make sure to save the current configuration before exiting the program.

\subsubsection{Edit menu}

The Edit menu provides options to select the measurement units (Units of Measure - English; Units of Measure - SI) and to set the probability distributions of the variable model parameters (Probability Distributions). The Edit menu options are shown in Figure 3-8. The two options available through the Edit menu are discussed in the following sub-sections. 
Figure 3-8. Edit menu options.

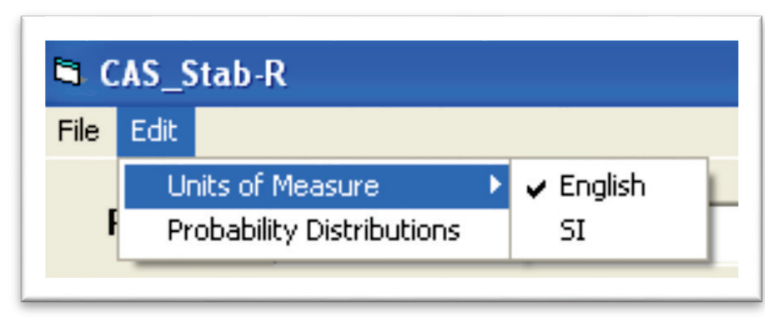

\subsubsection{Edit - Units of Measure}

Dimensions in CAS_Stab-R may be displayed in English units or System International (SI) units. Clicking the Edit menu item and moving the cursor over the Units of Measure item, the two choices for the system of units will appear as shown in Figure 3-8.

The currently selected system of units will be checked. In Figure 3-8, English units are the current system of units. Click on the desired units, English or SI, to choose the preferred measurement units for input and display values.

\subsubsection{Edit - Probability Distributions}

In order to view and edit the probability distributions assigned to the variables, choose the Edit - Probability Distributions option. A new window will appear on the screen as seen in Figure 3-9.

The variable properties are those that affect the amount of corrosion of the anchor cables, the anchor lock-off force, the silt and soils lateral pressure coefficients, and the cohesion, internal friction angle, and hydraulic conductivity of each material. The distribution values are assigned by selecting the option button for a property. If choosing an anchor, a soil layer or a material property, use the drop-down lists to choose the correct anchor, soil layer, or material number. The input boxes in the Distribution box to the right will be populated with the current distribution values for the selected property.

The material properties for the cohesion, $C$, and internal friction angle, $\phi$, are often related to some degree. CAS_Stab-R allows the level of correlation between these two variables to be specified in the C-Phi Correlation text box. Valid values for correlation lie in the range -1.0 to +1.0 . 
Figure 3-9. Probability Variable Input window.

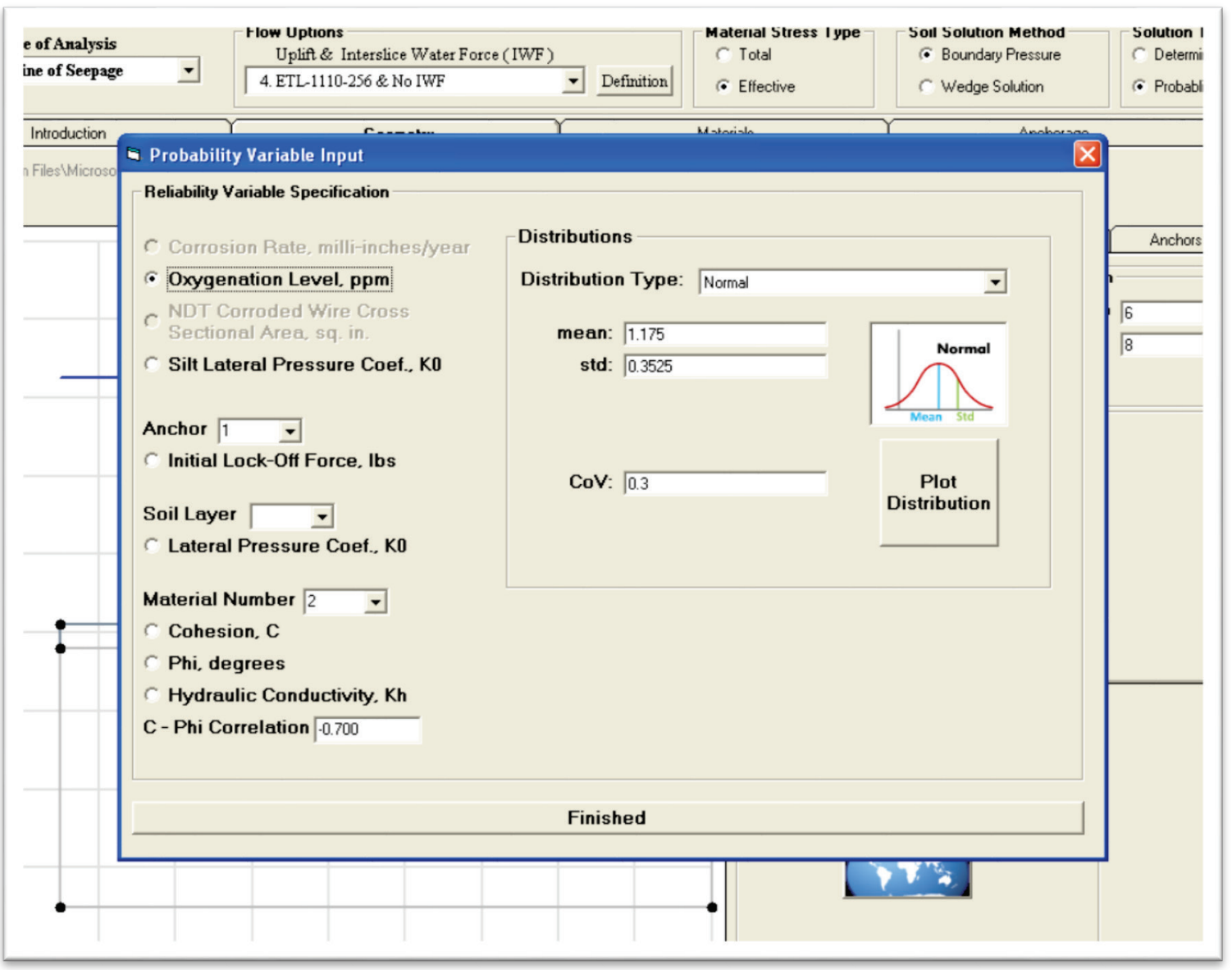

The Distributions box allows the user to modify the distribution parameters for a property. The type of distribution is selected using the drop-down list labeled Distribution Type. Currently the distribution types supported are Normal, Truncated Normal, Log-Normal, Truncated LogNormal, Uniform, and Triangular. These types of distributions are discussed in detail in Section 2.1.2 of this report. The values for the mean, standard deviation, start point, mid-point, and end point or the COV are assigned using the input boxes labeled as follows: mean:, std:, start, mid, end, and CoV:, respectively.

A probability distribution function curve or a cumulative distribution function curve of the currently selected values may be displayed by clicking the Plot Distribution button. Doing so will produce a new window as seen in Figure 3-10. This plot shows the probability (vertical axis) with respect to the variable's value (horizontal axis). 
Figure 3-10. Probability distribution function curve.

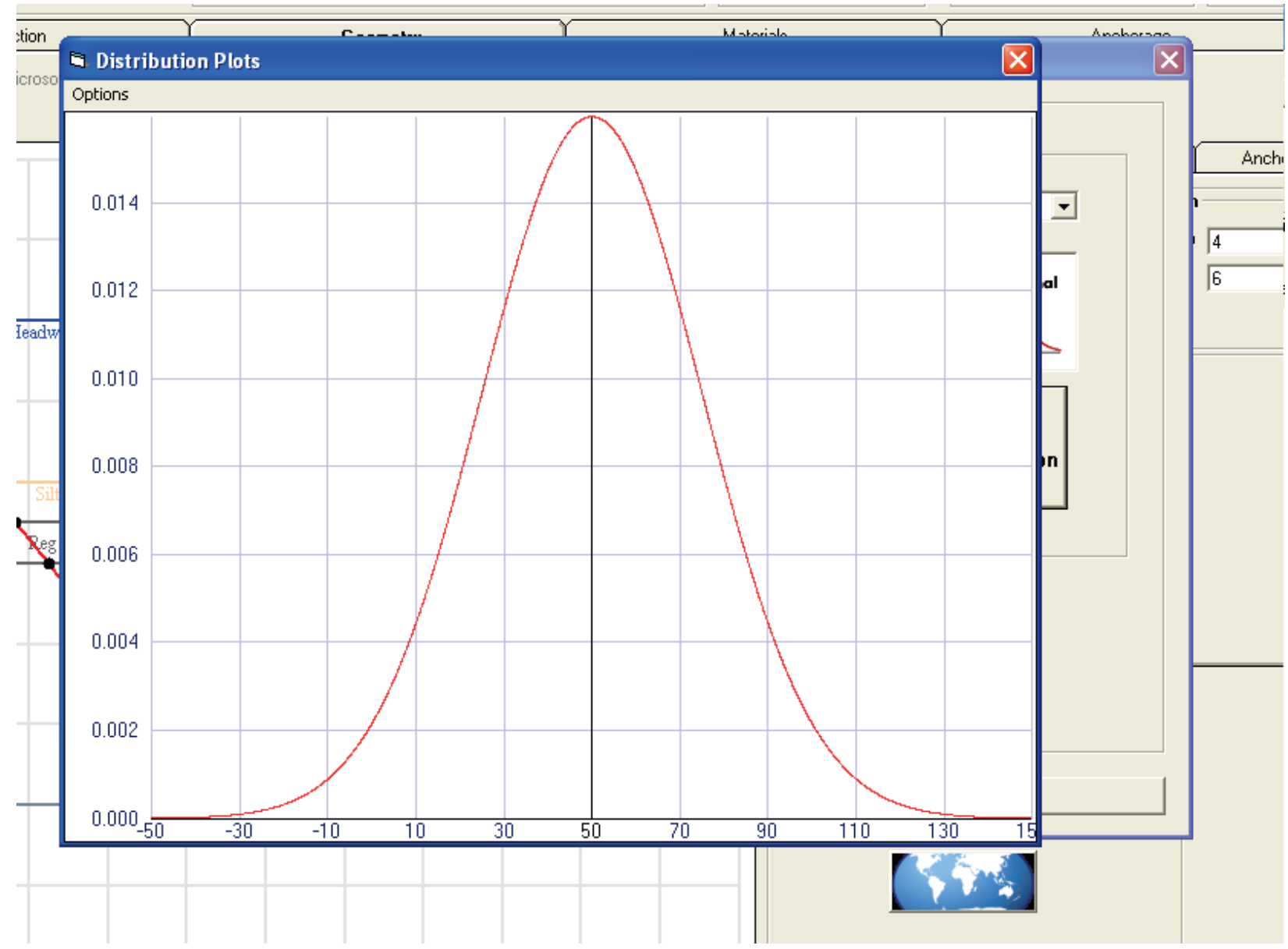

The CDF curve can be viewed by clicking the Options menu item located in the top left corner of the window and selecting the CDF option in the menu drop-down. The Options menu is shown in Figure 3-11. A sample cumulative distribution function curve is shown in Figure 3-12. Close this window by selecting the Exit option in the Options menu.

Figure 3-11. Distribution Plots-Options menu.

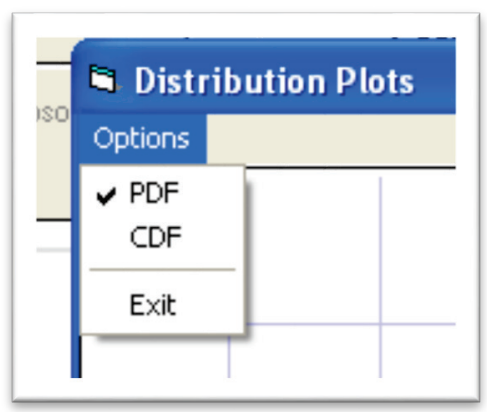


Figure 3-12. CDF curve.

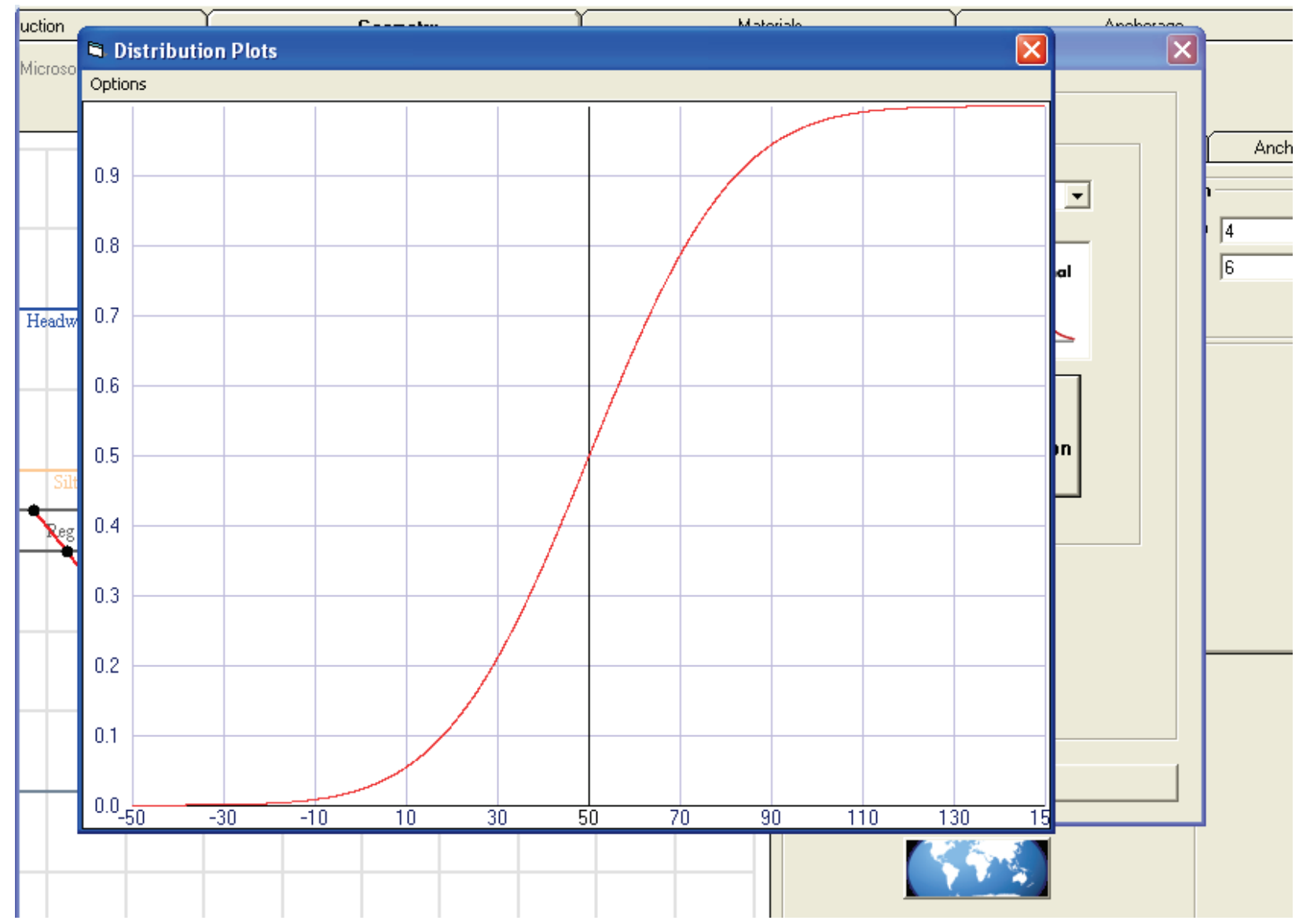

\subsubsection{Processing options}

The box located just below the Project input box (and shown in Figure 3-13) contains buttons to select options that are used in the analysis computations. These buttons are discussed later in sections 3.4.9 and following immediately after the Analysis tab section of the report.

Figure 3-13. Analysis options.

\begin{tabular}{|c|c|c|c|c|c|c|c|}
\hline \multicolumn{2}{|l|}{ Type of Analysis } & \multirow{2}{*}{$\begin{array}{l}\text { Flow Options } \\
\text { Uplift \& Interslice Water Force (IWF) } \\
\begin{array}{|l}\text { 4. ETL-1110-256 \& No IWF }\end{array}\end{array}$} & \multirow[b]{2}{*}{ Definition } & \multirow{2}{*}{$\begin{array}{l}\text { Material Stress Type } \\
\subset \text { Total } \\
\text { C Effective }\end{array}$} & \multirow{2}{*}{$\begin{array}{l}\text { Soil Solution Method } \\
\text { - Boundary Pressure } \\
r \text { Wedge Solution }\end{array}$} & \multirow{2}{*}{$\begin{array}{l}\text { Solution Type } \\
\text { C Deterministic } \\
\text { C Probablilistic }\end{array}$} & \multirow{2}{*}{$\begin{array}{l}\text { Return to } \\
\text { Main }\end{array}$} \\
\hline Stability \& Line of Seepage & ت & & & & & & \\
\hline
\end{tabular}

\subsubsection{Introduction tab}

The Introduction tab is displayed upon selection of the Analysis of Structural Stability Against Sliding option. This tab's display is shown in Figure 3-2. A sample model is shown on the left side of the tab. A Corroded 
Cable Capacity curve that displays the relationship of the cable capacity to the corroded wire short axis diameter is shown on the right side of the tab.

\subsubsection{Geometry tab}

Selecting the Geometry tab reveals the controls shown in Figure 3-14. Figure 3-14 also lists the various sections of this tab. The sections are the following:

- Geometry Definitions Tabs - tabbed input area for defining the model

- Cursor Coordinates - displays of the $x$ - and $y$-coordinates of the current cursor position

- Message Area - section where miscellaneous information is shown (such as currently selected node coordinates, nodes added to definitions)

- Model Extents Box - area where the range values of the Geometry Display are defined

- Geometry Display - area that displays the scale model drawing.

The functions of these sections will be discussed in the following report sections.

Figure 3-14. CAS_Stab-R structural stability Geometry tab.

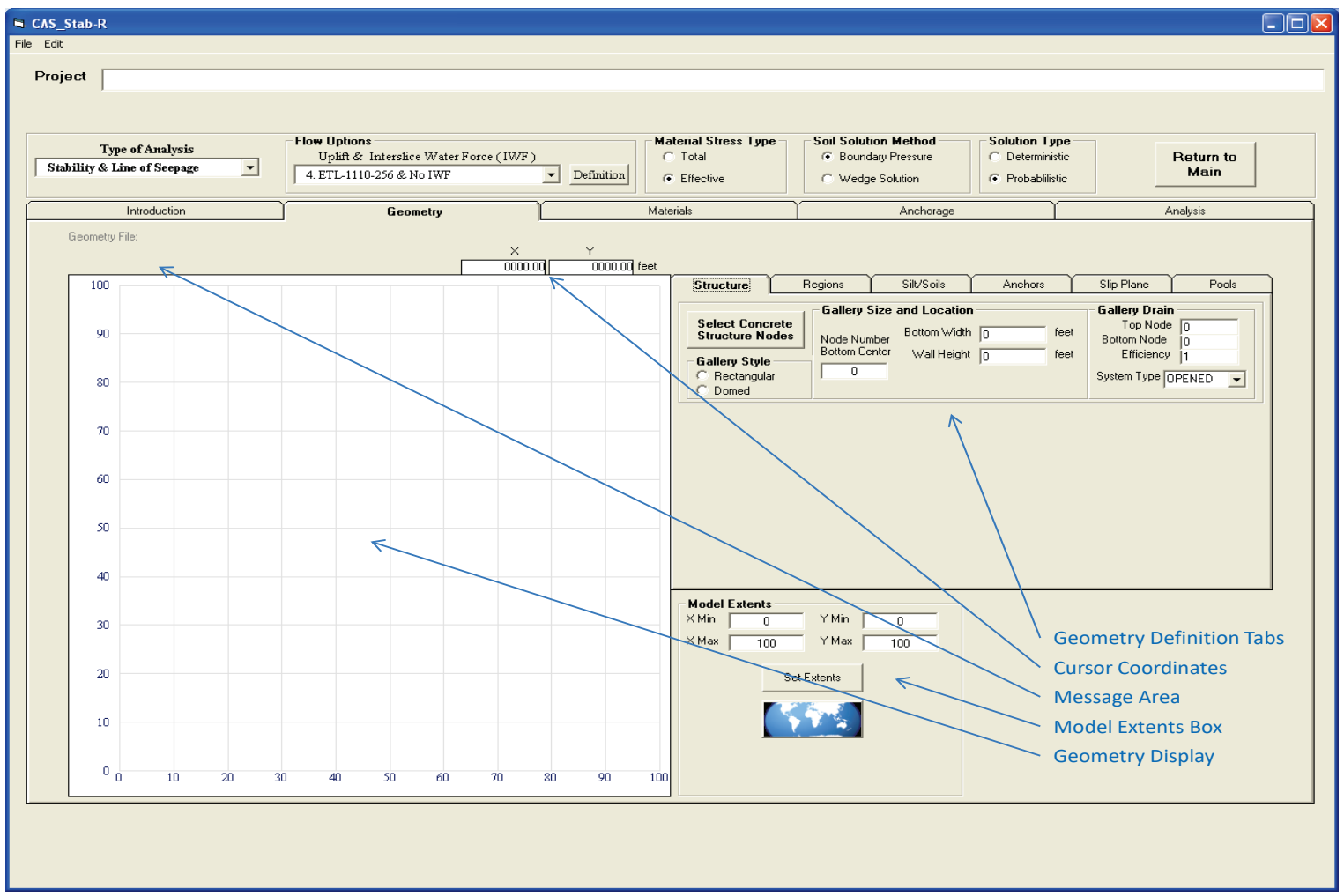




\subsubsection{Model Extents Box and Zoom capability}

The Model Extents Box is located to the right of the Geometry Display. At startup, the model extents are set to minimum values of $o$ and maximum values of 100 for the $x$ and $y$ axes. This will be reflected in the Model Extents Box. The input boxes labeled X Min, X Max, Y Min, and Y Max will contain the values $0,100,0$, and 100 , respectively. If a node coordinate is entered that is outside of this range, the program will automatically extend the model extents to include the node. The model extent boxes will be updated to reflect the change. The user may manually change the model extents by entering new values into the model extent input boxes and clicking the button labeled Set Extents.

The Model Extents Box also contains a Zoom To World button that is shown in Figure 3-15. Clicking this button causes the program to determine the minimum and maximum $x$ - and $y$-coordinate values of all the defined nodes. The model extents are then set to appropriate values to include all nodes. The model extent input boxes will be updated, and the Geometry Display will be updated to reflect the changes.

Figure 3-15.

Zoom To World button.

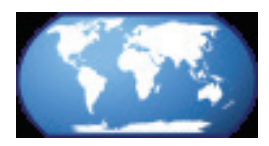

CAS_Stab-R provides the ability to select an area of the Geometry Display to magnify. Perform the following steps to zoom in on an area:

1. Position the cursor at a corner of the area to be zoomed.

2. Depress and hold the left mouse button.

3. Move the mouse to the opposite diagonal corner of the zoom area.

4. Release the mouse button.

As the mouse is moved during Step 3 above, a box outlining the selected area will appear in the Geometry Display as shown in Figure 3-16. Upon release of the mouse button, the Geometry Display will show only the selected area as shown in Figure 3-17. Note that the zoomed area displayed may be slightly different than that selected. The program maintains equal $x$ - and $y$-axis increments so that proper model perspective is maintained. 
Figure 3-16. Geometry Display with Zoom Box.

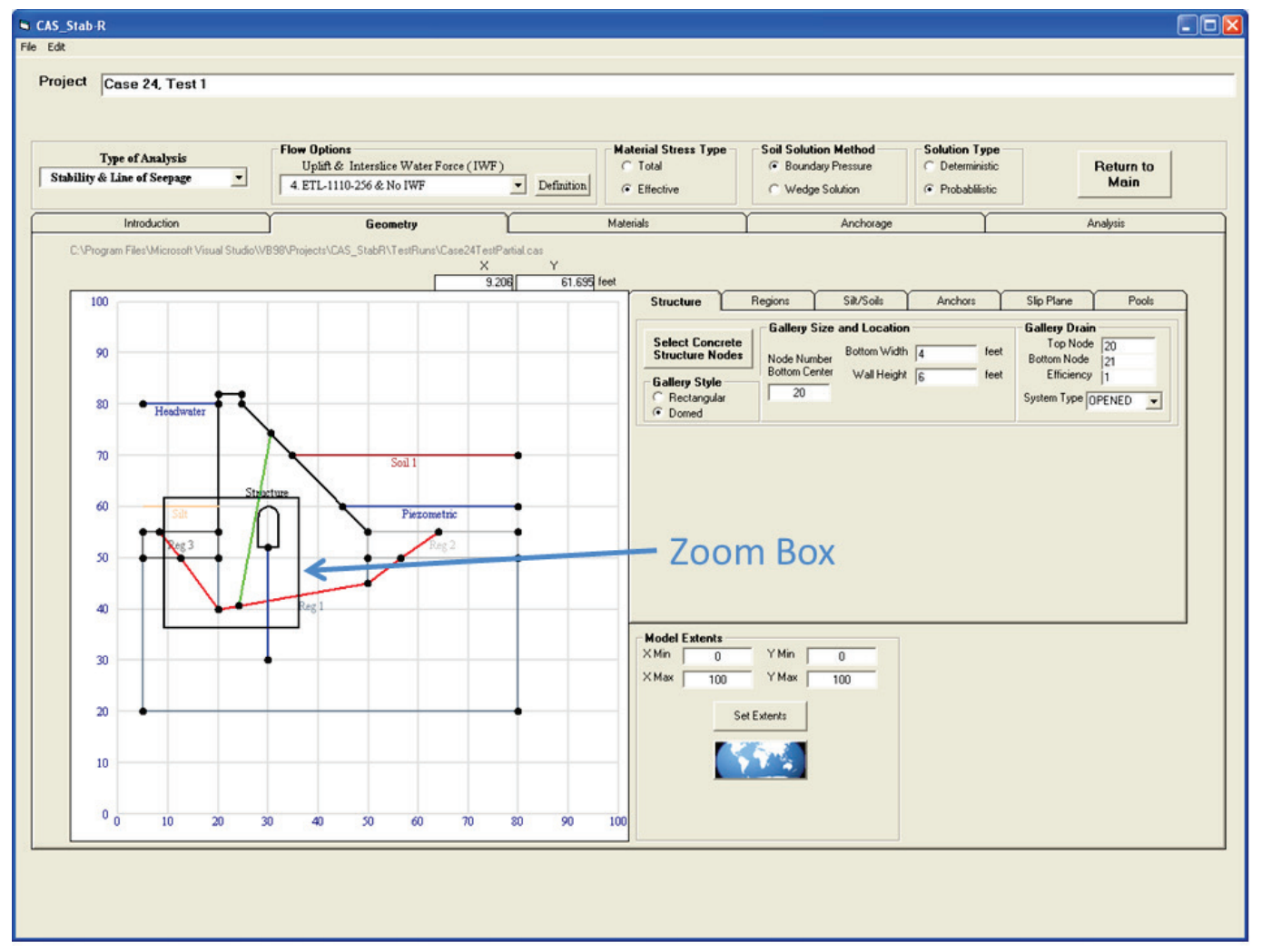

Figure 3-17. Geometry Display with Zoomed Area.

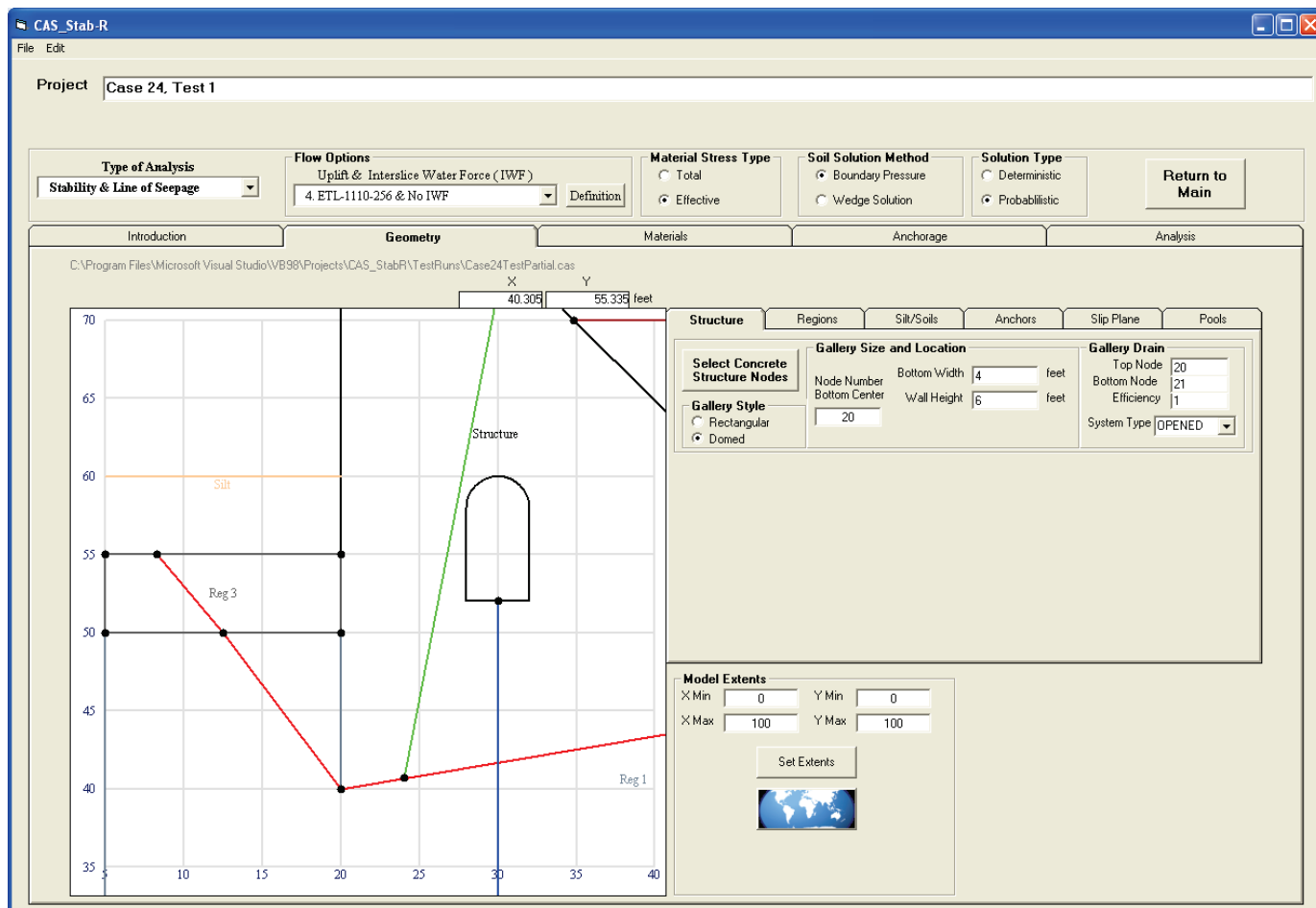


Two methods are available to return to the full model display. One method is to click the Set Extents button. This will result in the update of the Geometry Display with $x$ - and $y$-coordinate values determined by the model extents input boxes. Therefore, if the user has manually entered new values into these boxes after zooming the display, the result may not include the entire model. The entire model display should result if the model extents boxes have not been altered. The other method is to click the Zoom To World button. The behavior of the program to this input is described in the second paragraph of this section.

\subsubsection{Node entry and editing}

The process of building the model starts by entering nodes that will define boundary endpoints for the various model components. Nodes are entered by right-clicking in the Geometry Display to obtain the popup menu as shown in Figure 3-18. Select the top popup menu option Add Node. A popup window will appear that displays the current $\mathrm{X}, \mathrm{Y}$ coordinate values as shown in Figure 3-19. The X,Y coordinates may be modified by entering desired values into the appropriate text boxes or left as is. Click OK when satisfied with the values selected. A point will appear at the selected coordinate as shown in Figure 3-20. Continue adding nodes as needed to define the various nodal boundaries of the model.

To change the location of an existing node, place the cursor over the node so the node is highlighted in red as shown in Figure 3-21. The coordinate values of the highlighted node are indicated at the top of the Geometry Display window in the Message Area. Right click the node to display the popup menu (also shown in Figure 3-21). Note that a different set of options are enabled in the popup menu due to the highlighted node. Select the popup menu option Edit Node. The window as shown in Figure 3-19 will appear. Modify the $\mathrm{X}$ and $\mathrm{Y}$ values as necessary and select $\mathrm{OK}$. The node will be moved to the new location.

Node numbers can be shown in the Geometry Display by right-clicking in the display and selecting Show Node Numbers from the popup menu as shown in Figure 3-22. To remove the node numbers from the display, click the right mouse button and select the Hide Node Numbers option. Figure 3-23 contains a display with the node numbers shown. 
Figure 3-18. Geometry Display with popup menu.

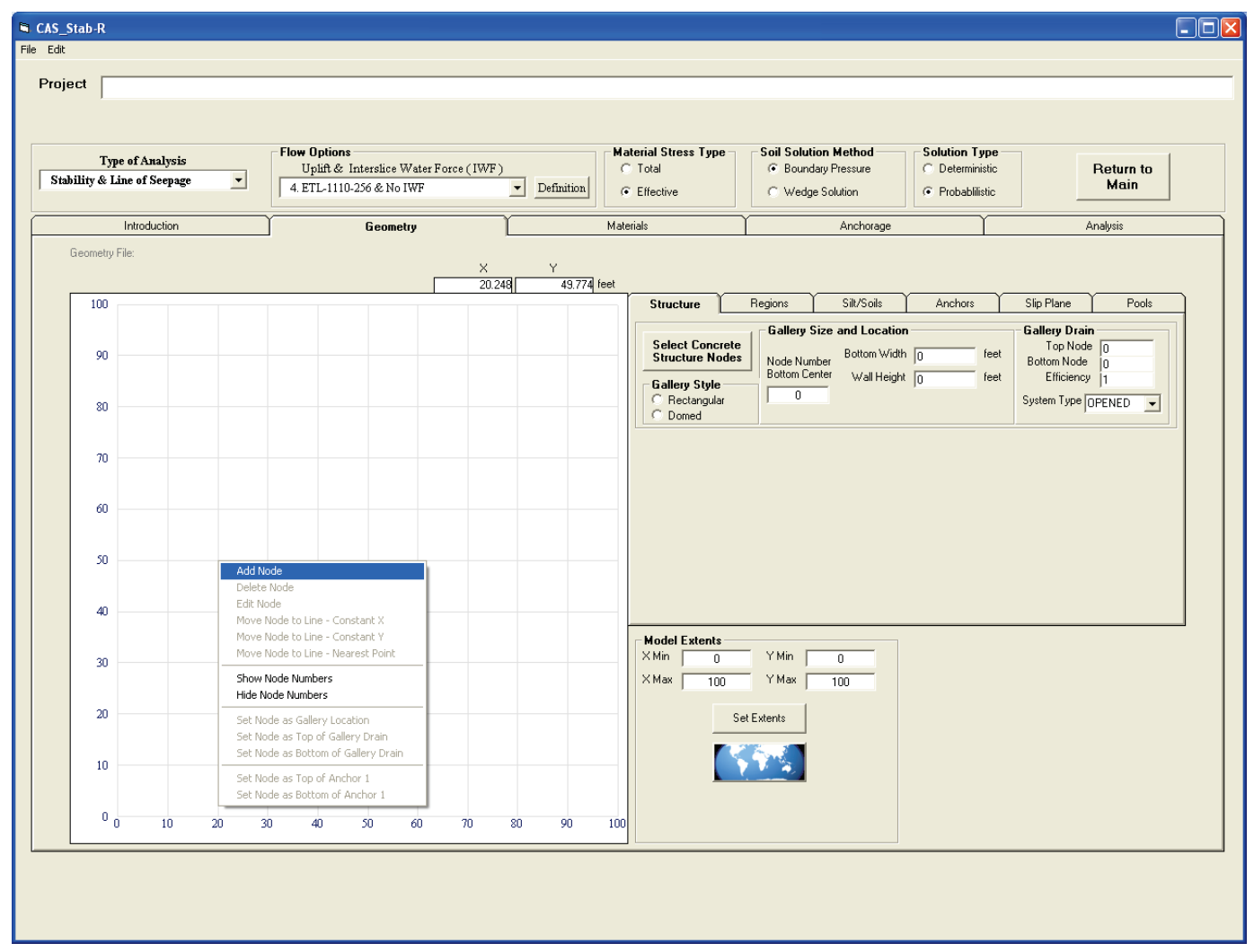

Figure 3-19. Node coordinate entry window.

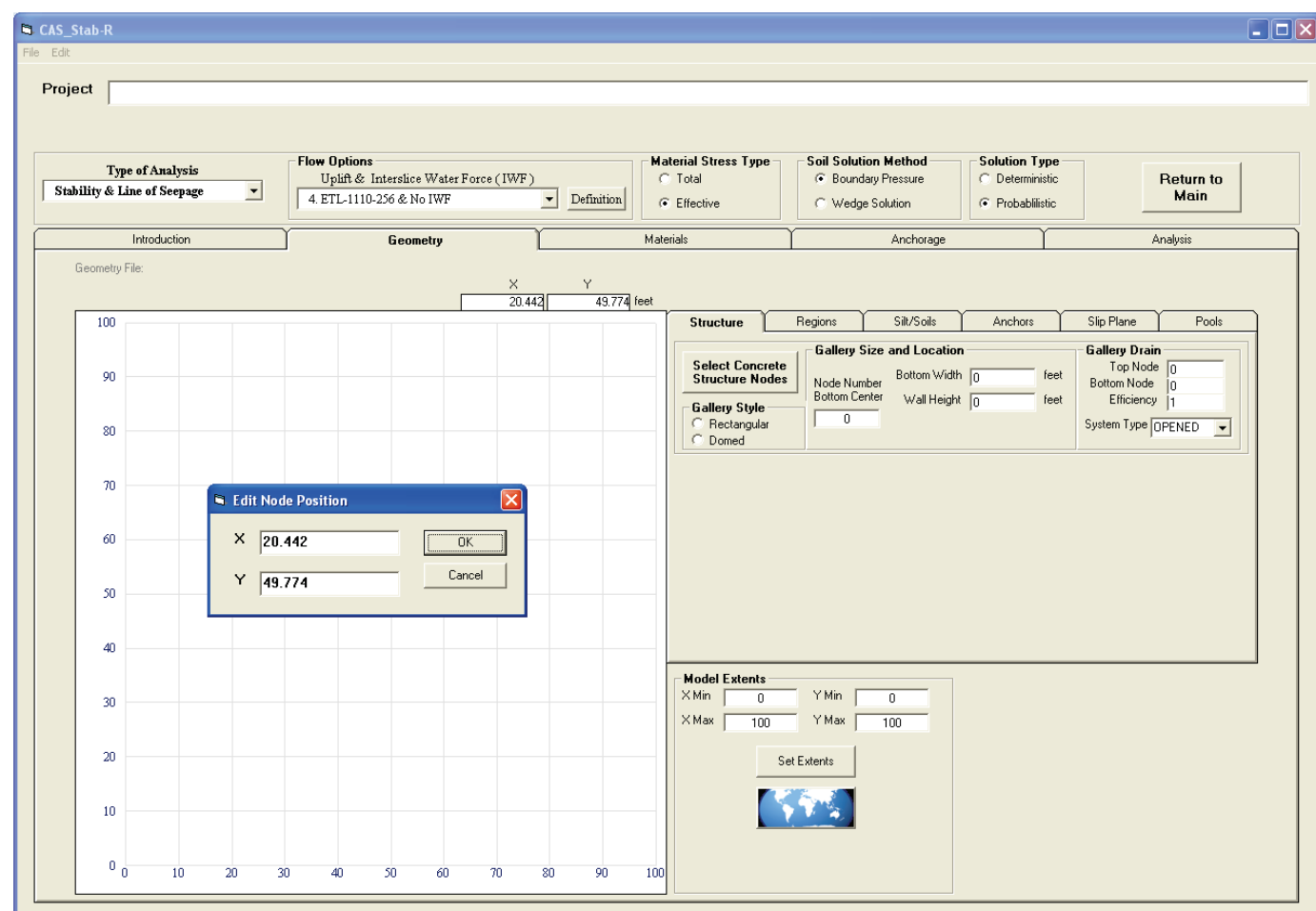


Figure 3-20. Geometry Display with a node at coordinate $(20,50)$.

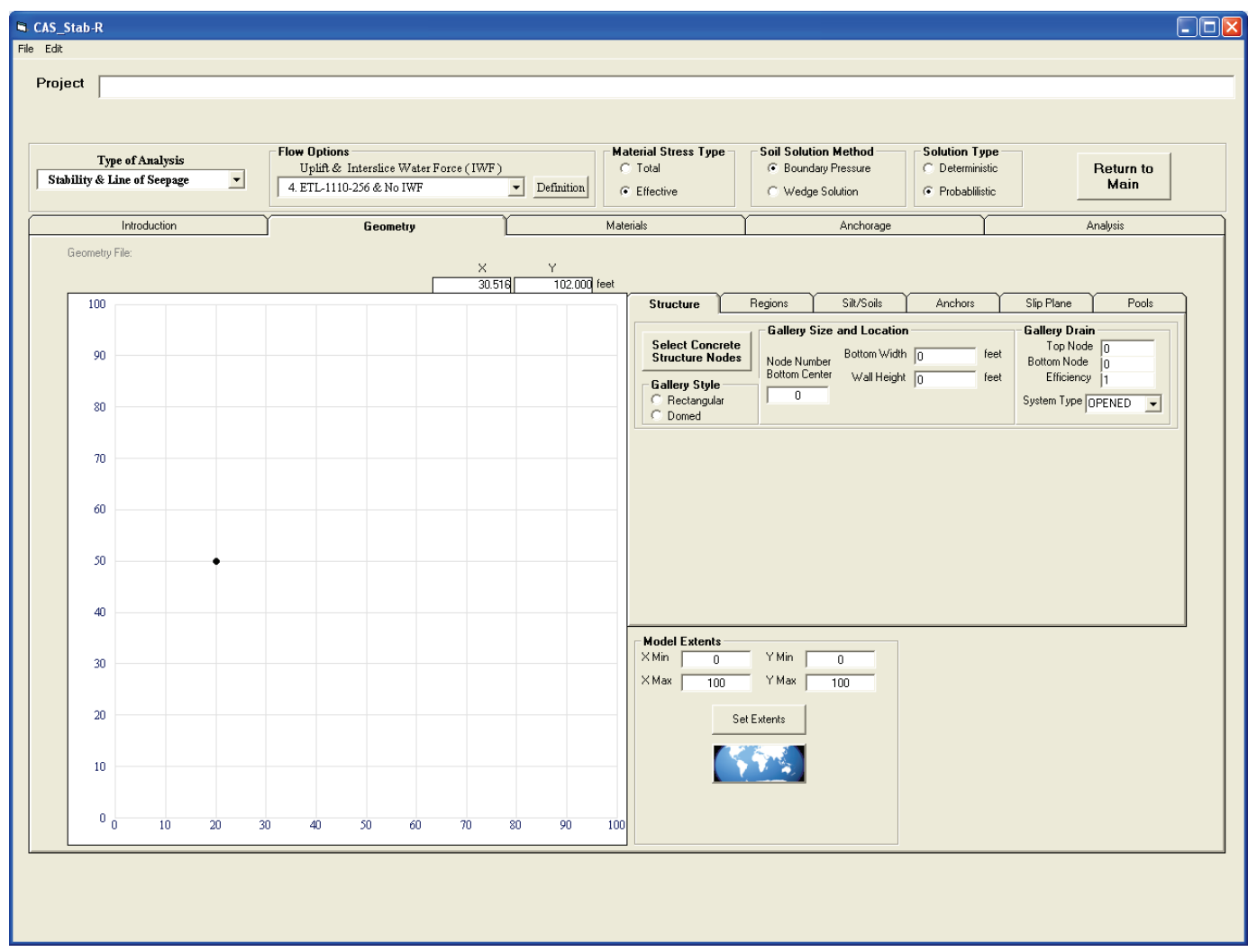

Figure 3-21. Geometry Display with a highlighted node.

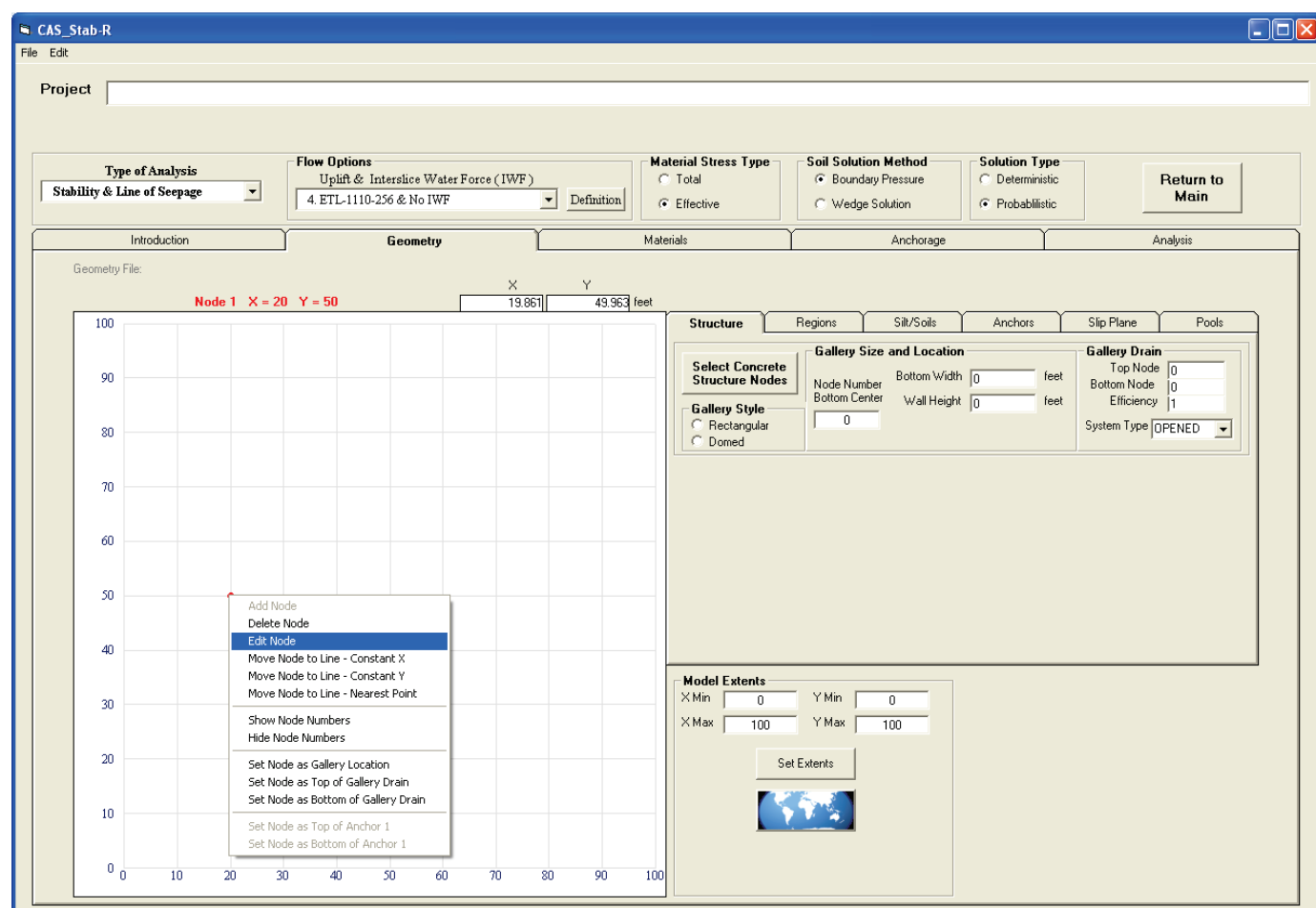


Figure 3-22. Menu option to display node numbers.

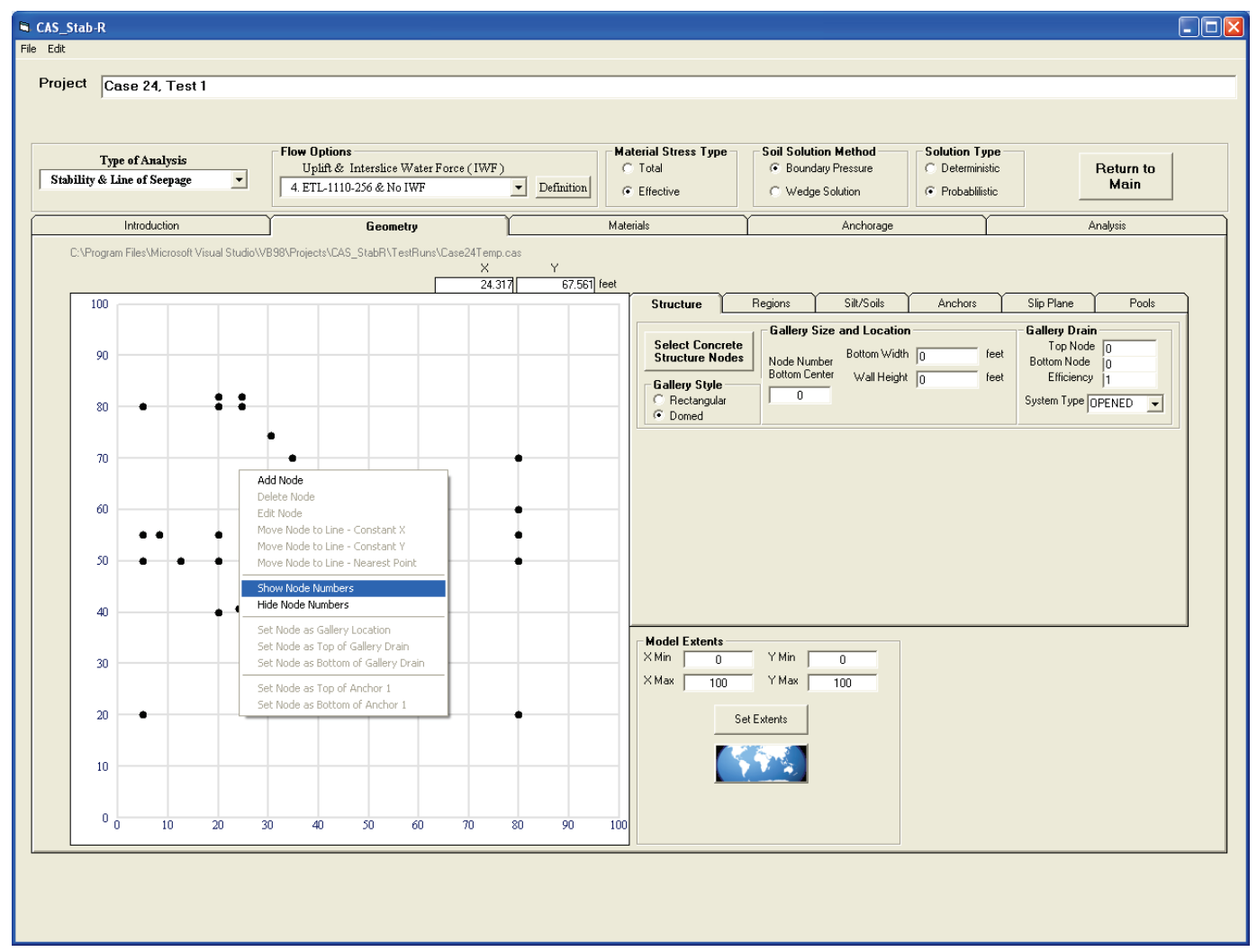

Figure 3-23. Geometry Display with node numbers shown.

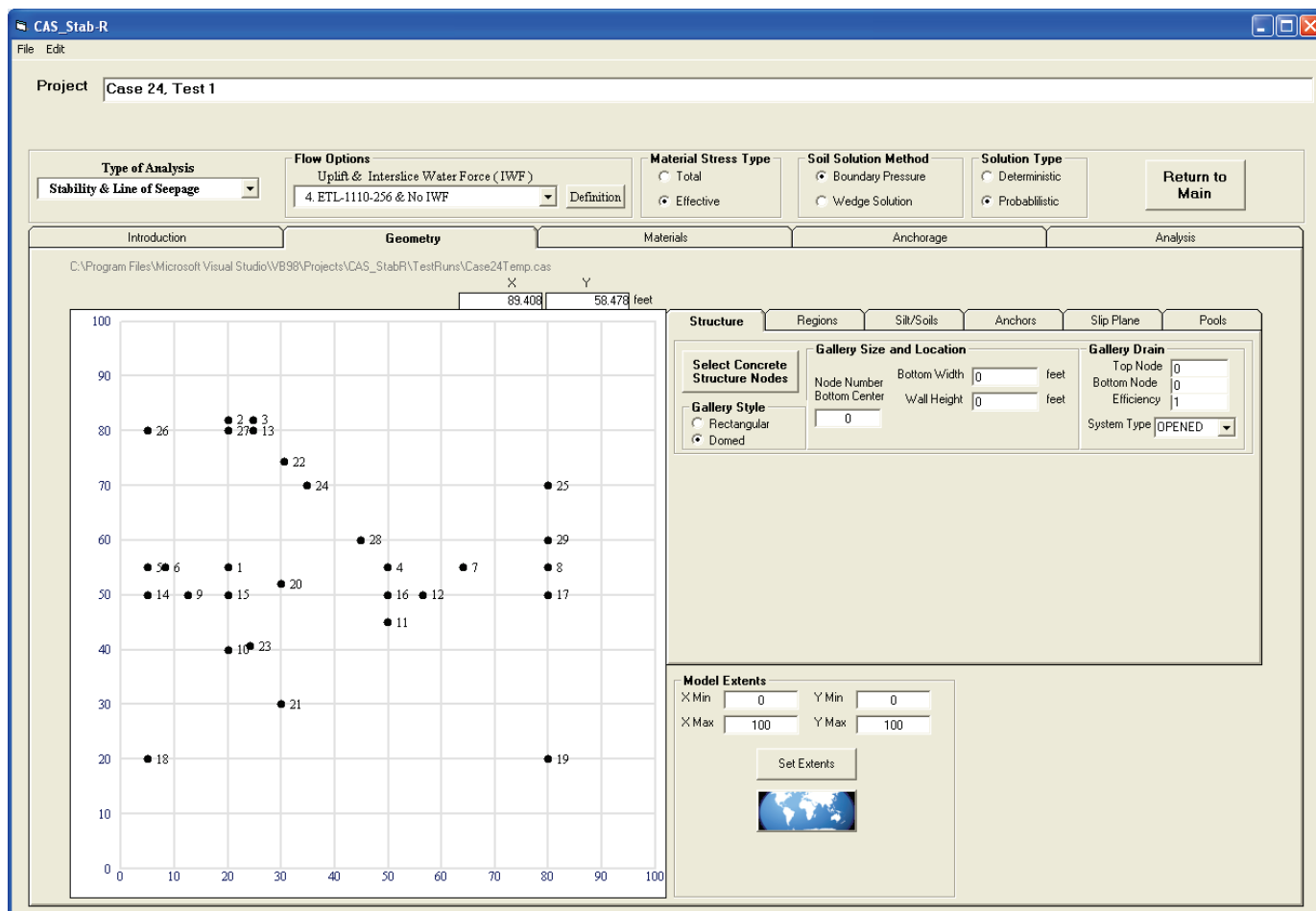




\subsubsection{Structure tab}

The nodes that define the structure are selected by working in the Structure tab to the right of the Geometry Display. This tab is shown in Figure 3-23 above. Clicking the Select Concrete Structure Nodes button starts the process. The message box shown in Figure 3-24 provides instructions for how to select nodes in the proper order to define the structure. Position the cursor over the first node (structure heel) so that it is highlighted. Click using the left mouse button. The status message in the Message Area above the Geometry Display will indicate this node has been added to the structure until the left mouse button is released. Continue to add nodes in a counterclockwise direction until the final node is selected. An orange line will be displayed to indicate the shape of the structure with the selected nodes as if the selection were ended as shown in Figure 3-25. When the final node has been selected, click the End Selection button, and the closed structure will be shown. The structure shown in Figure 3-26 was created by selecting nodes 10,11, 4, 13, 3, 2, and 1 in the specified order.

Figure 3-24. Structure node selection message box.

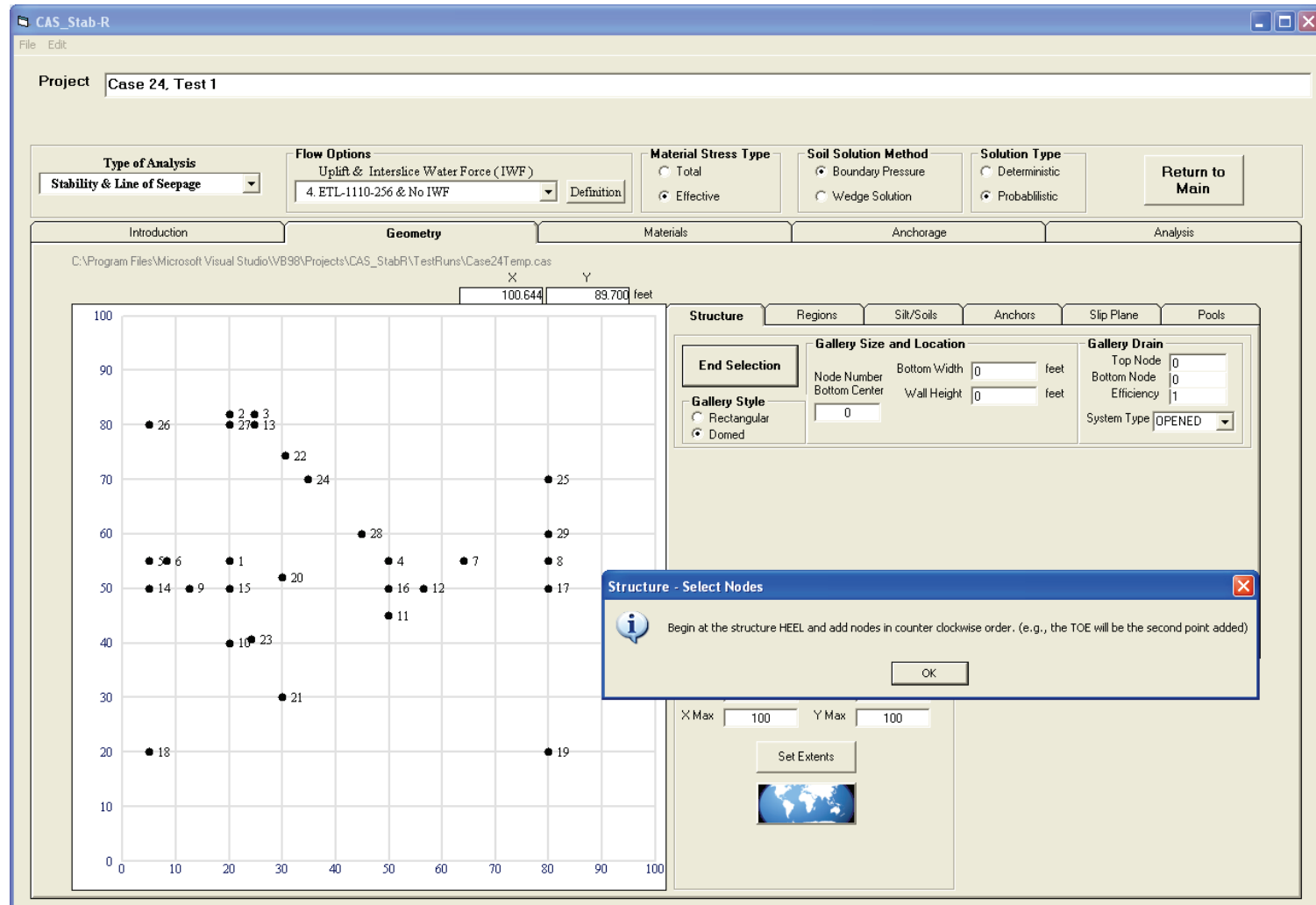


Figure 3-25. Geometry Display with partially defined structure.

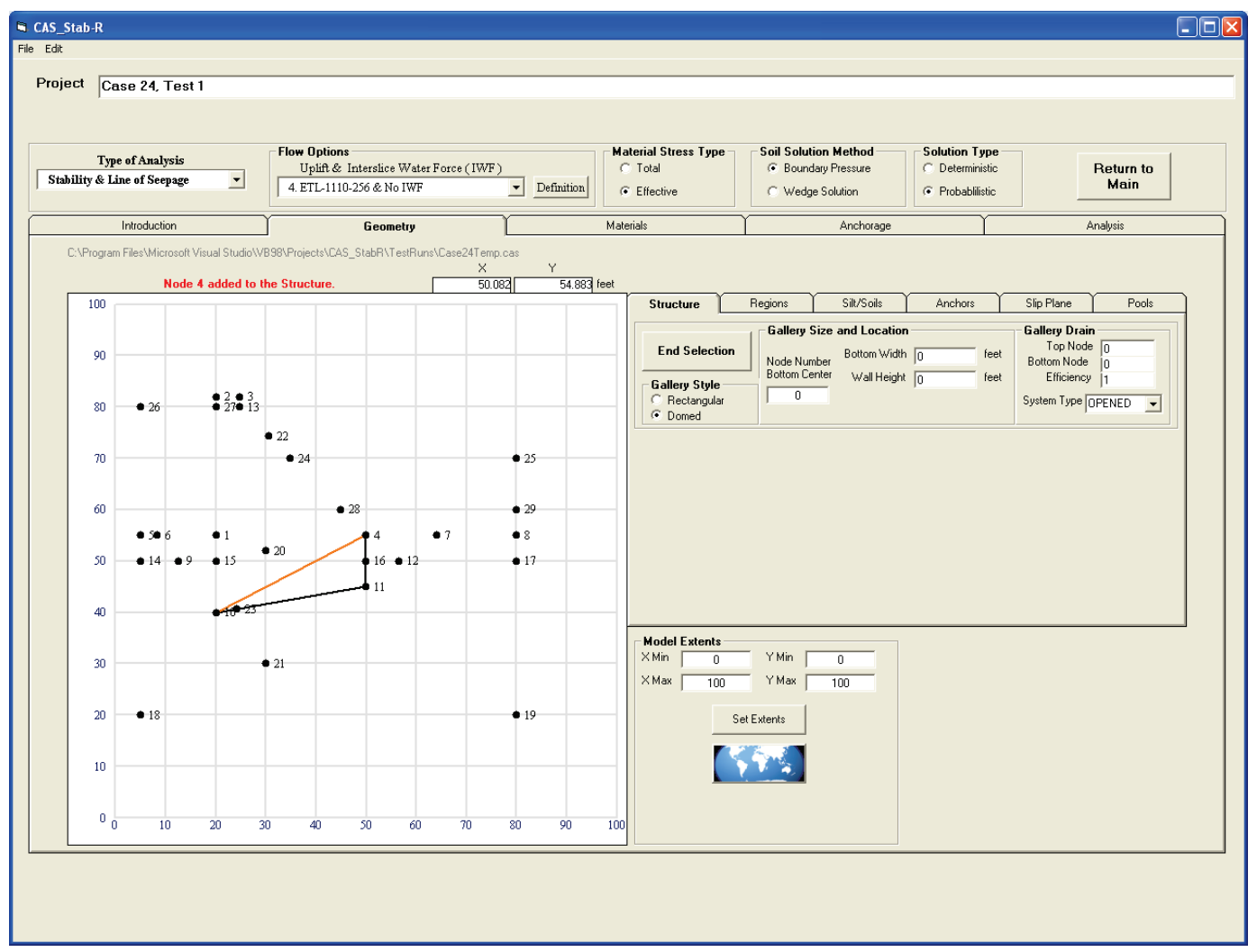

Figure 3-26. Geometry Display with a defined structure.

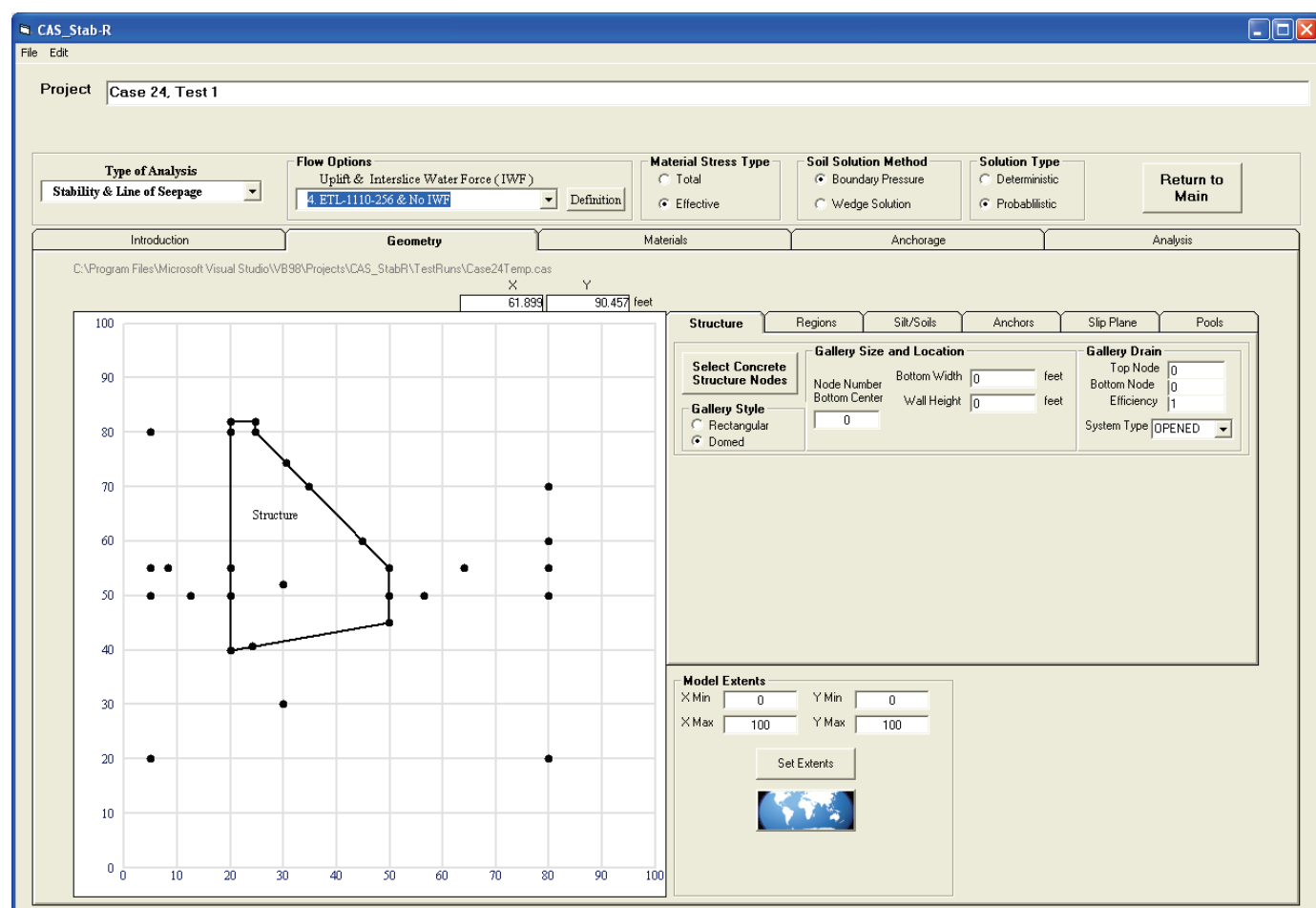




\subsubsection{Gallery definition}

Controls to define the structure gallery are on the Structure tab to the right of the Geometry Display. Select the type of gallery as domed or rectangular by choosing the Domed or Rectangular radio button, respectively. Indicate the width and height of the gallery by entering the dimensions into the appropriate input boxes. If the gallery is domed, the radius of the dome is set equal to one-half of the gallery width. Two methods are available to specify the location of the gallery. One method is to enter the node number for the bottom center of the gallery into the Location text box. The other method is to position the cursor over the node at the bottom center of the gallery in the Geometry Display. When the node is highlighted, right-click the node and select the Select Node as Gallery Location option from the popup menu as shown in Figure 3-27. The Node Number Bottom Center text box will be populated with the selected node number.

Figure 3-27. Select Node as Gallery Location popup option.

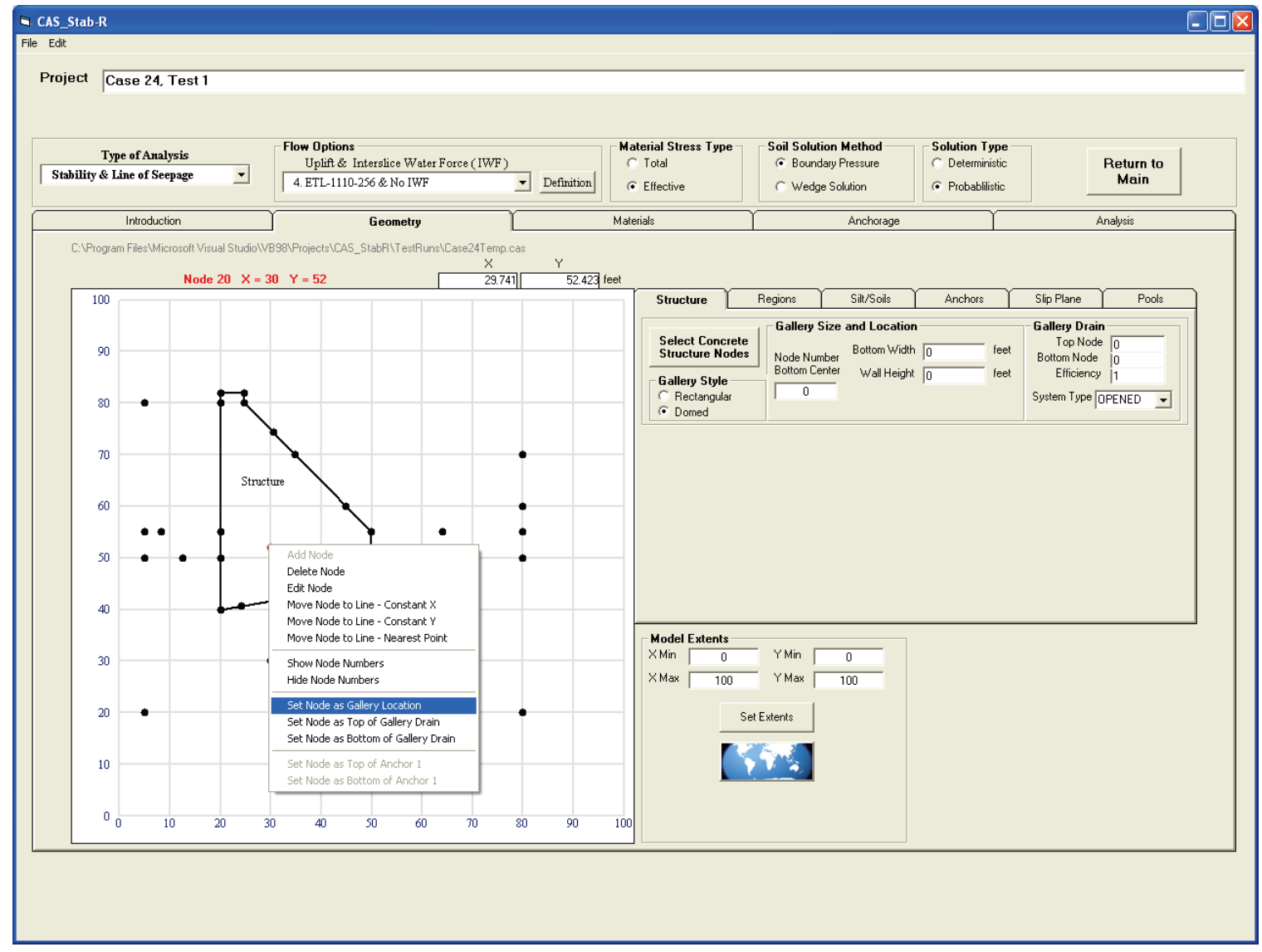


The gallery in Figure 3-28 was created by specifying a Bottom Width of 4 feet ( $\mathrm{ft}$ ), Wall Height of $6 \mathrm{ft}$, clicking the Domed radio button and selecting node 20 as the Node Number Bottom Center.

Figure 3-28. Geometry Display with a domed gallery.

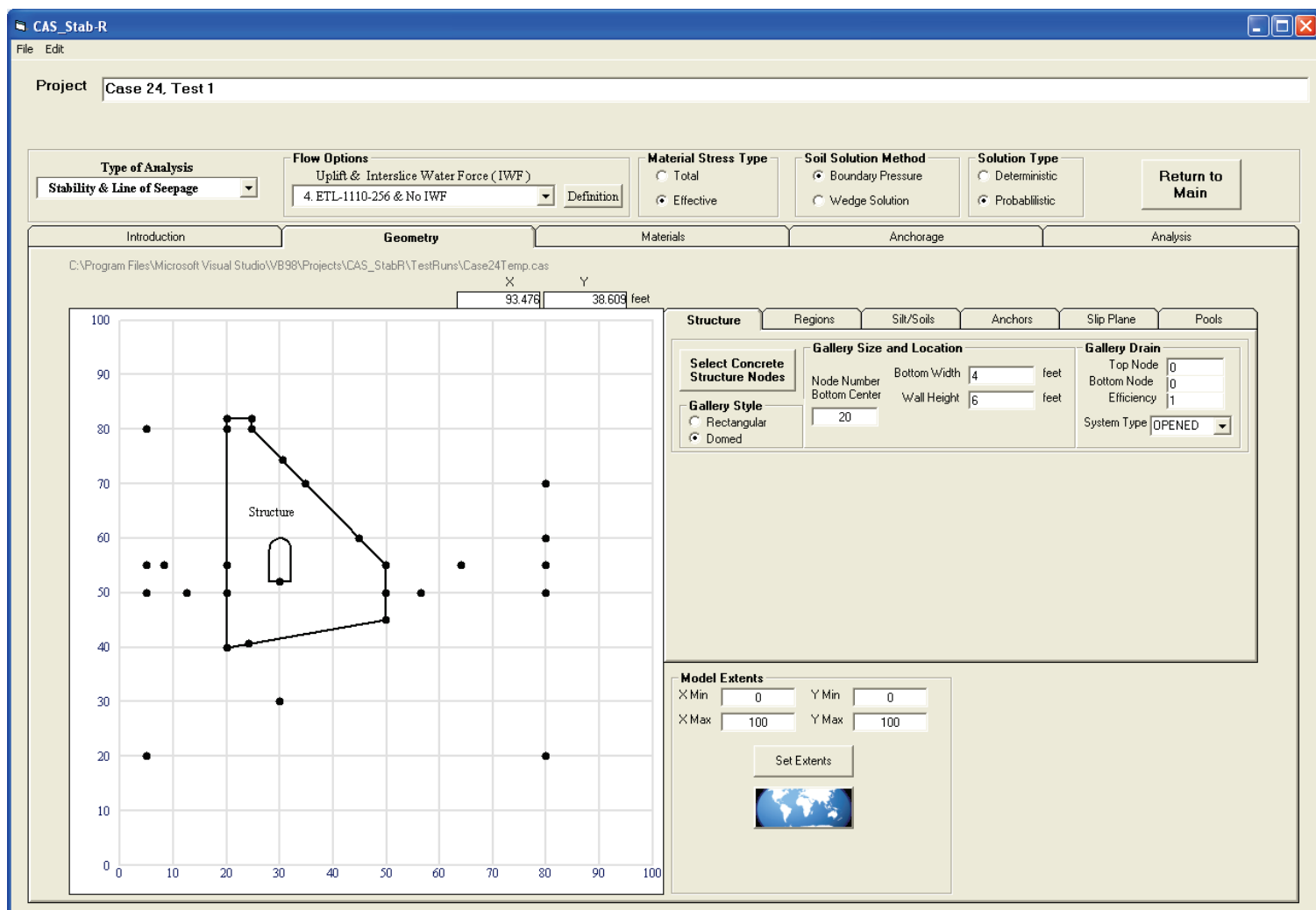

\subsubsection{Gallery Drain specification}

The gallery drain, if present, is specified by selecting a node as the top of the drain and a node as the bottom of the drain. To specify the node for the top of the drain, enter a node number into the Drain Top Node text box in the Gallery Drain box on the Structure tab to the right of the Geometry Display. An alternate method is to highlight the node, click the right mouse button, and select the Set Node as Drain Top option from the popup menu as shown in Figure 3-29. 
Figure 3-29. Set Node as Top of Gallery Drain popup menu option.

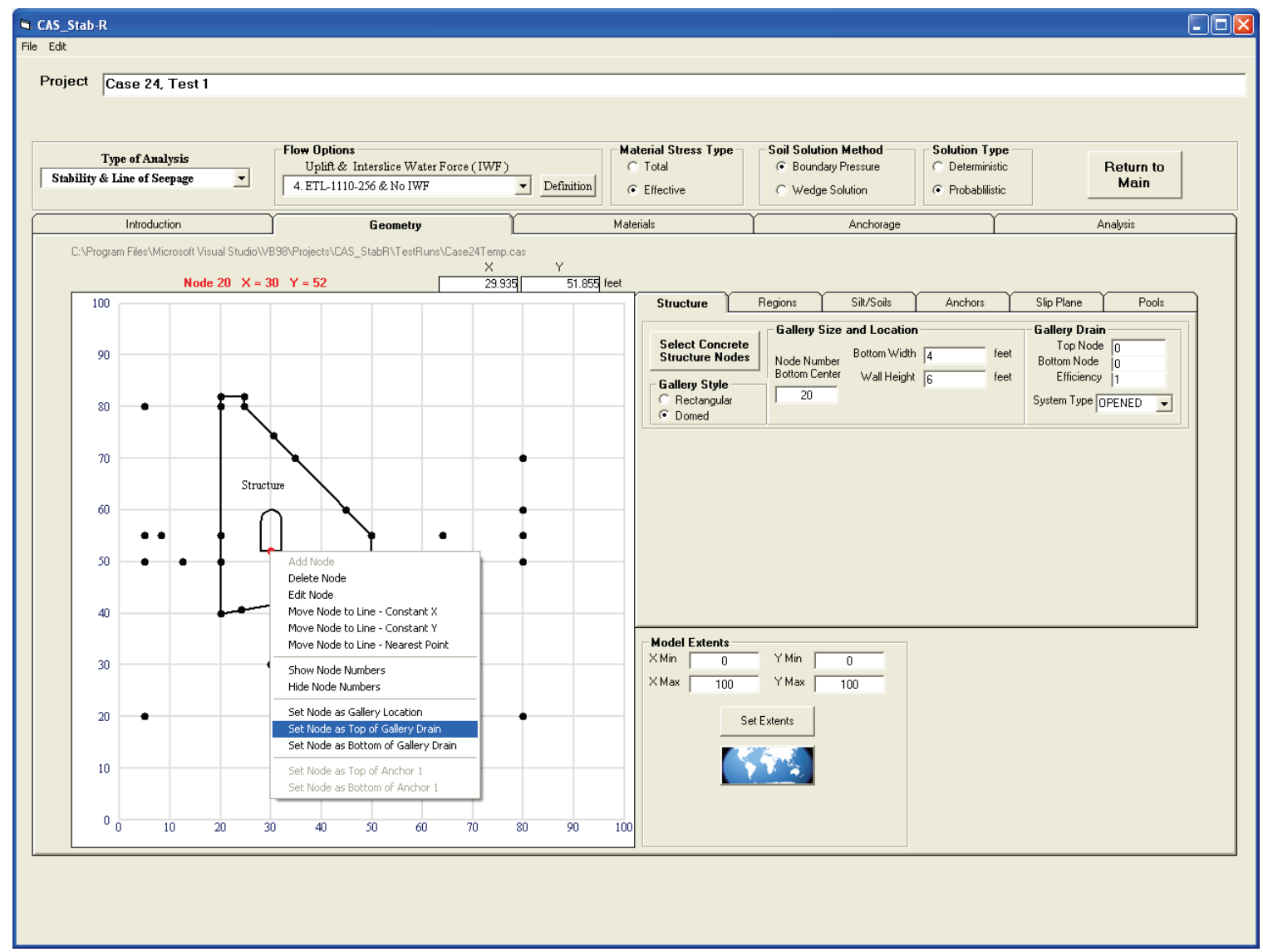

The node for the bottom of the drain is specified in a like manner. When the top and bottom nodes have been selected, the drain line will be indicated by a blue line as seen in Figure 3-30. The drain in Figure 3-30 was specified by choosing node 20 as the top of the drain and node 21 as the bottom of the drain.

The type of drain (CLOSED, OPENED, or NO DRAIN) is chosen using the drop-down list box labeled System Type in the Gallery Drain box on the Structure tab to the right of the Geometry Display.

Open drainage gallery: In an open drainage gallery system, foundation drainage water collected from a shallow channel over the drain openings in the gallery floor is drained using an "open drainage gallery system." This system extends from this shallow gallery floor channel out to the downstream face of the dam under gravity flow. If not "blocked" (e.g., by a one-way valve), this gallery drainage feature will also allow high tailwater flow back up this same drainage feature and into the gallery. 
Closed drainage gallery: For a closed drainage gallery system, the foundation drainage water flows through a shallow channel over the drain openings in the gallery floor. The drains empty into a sump that has a pump. The drainage in the sump is pumped out of the gallery. A closed drainage gallery system requires that the downstream outlet feature be high enough that tailwater cannot back up into this outlet, overwhelming the sump pump and flooding the gallery.

CAS_Stab-R uses the same drainage models as GDLAD_Foundation software (Ebeling et al. 2012). Detailed discussions on open and closed drainage gallery systems are provided in Section 1.2.1 of Ebeling et al. (2012) and through the use of detailed figures in Sections 6.3 through 6.5 for flow options 4, 5, and 6 .

Drain efficiency: The drain efficiency is a non-site specific parameter that describes the amount of control that the drain has over the magnitude of the uplift pressure acting at the base of the structure. Drains act to reduce the magnitude of the uplift pressure resulting from differential head from the upstream to downstream side of the structure. It is part of the input of the data box labeled Gallery Drain in Figure 3-29. Drain efficiency is expressed as a percentage ranging from $o$ to 100 . A value of Drain efficiency $=100$ corresponds to the case of the drains being fully effective (i.e., 100\%) and a value of Drain efficiency $=0$ corresponds to the case of the drains being fully clogged and ineffective. U.S. Army Corps of Engineers Engineer Manuals 1110-2-2200, Gravity Dam Design, and 1110-2-2100, Stability Analysis of Concrete Structures, restrict the value of Drain efficiency to no greater than 50\%. These manuals state that if foundation testing and flow analysis provide supporting justification, the drain efficiency can be increased beyond 50\%. The analysis and/or design documentation must contain supporting data to justify the Drain efficiency value used. 
Figure 3-30. Geometry Display with a gallery drain.

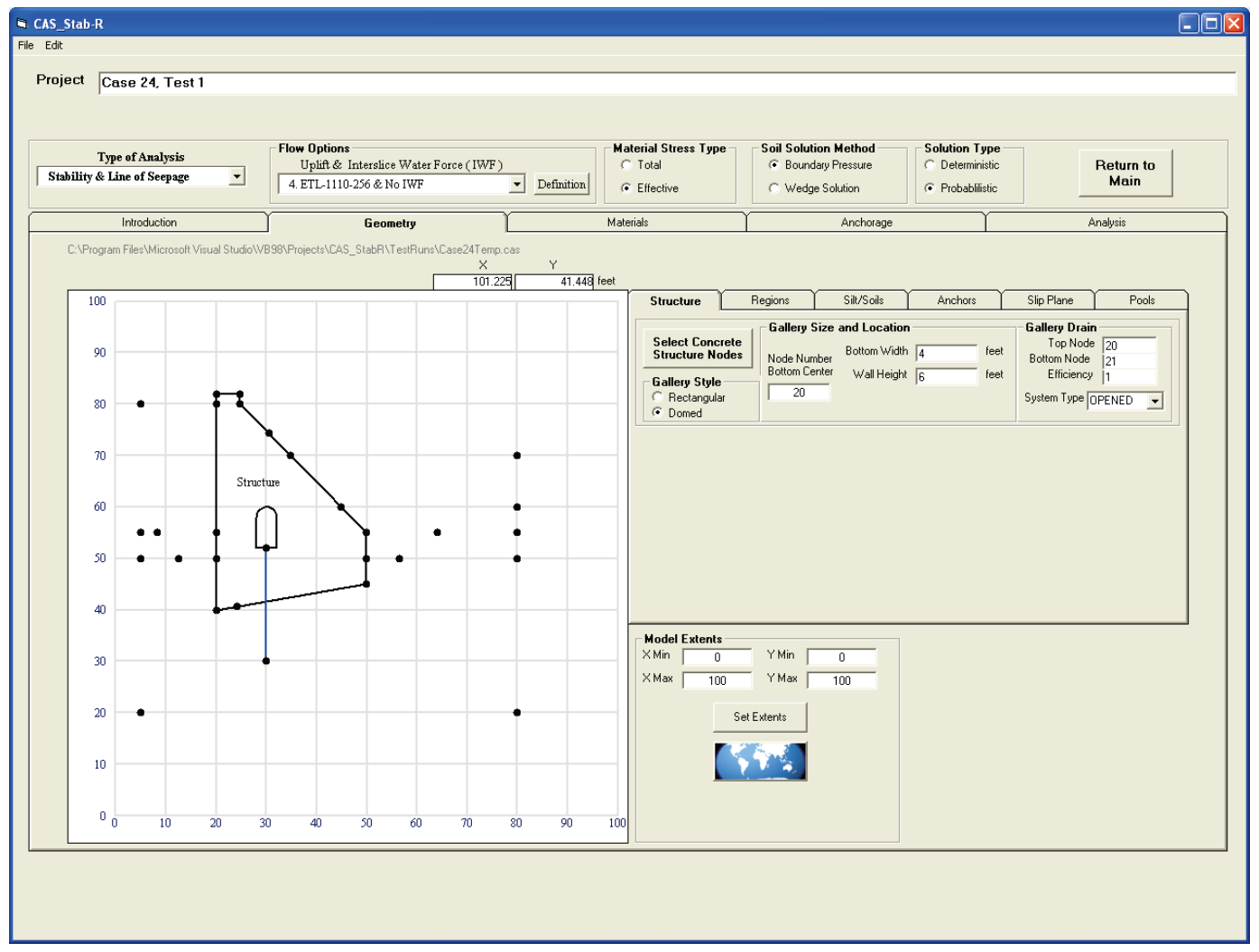

\subsubsection{Regions tab}

Regions where materials can be applied are added to the model using the controls on the Regions tab to the right of the Geometry Display. This tab is shown in Figure 3-31.

In the upper left corner of the tab is a display box labeled Number of Defined Regions that informs the user of the current number of defined regions in the model. Immediately below the display box is a button labeled Add a Material Region. Click this button to add a region to the model. A window with instructions for selecting the nodes to define the region will pop up on the screen as seen in Figure 3-32. 
Figure 3-31. Regions tab.

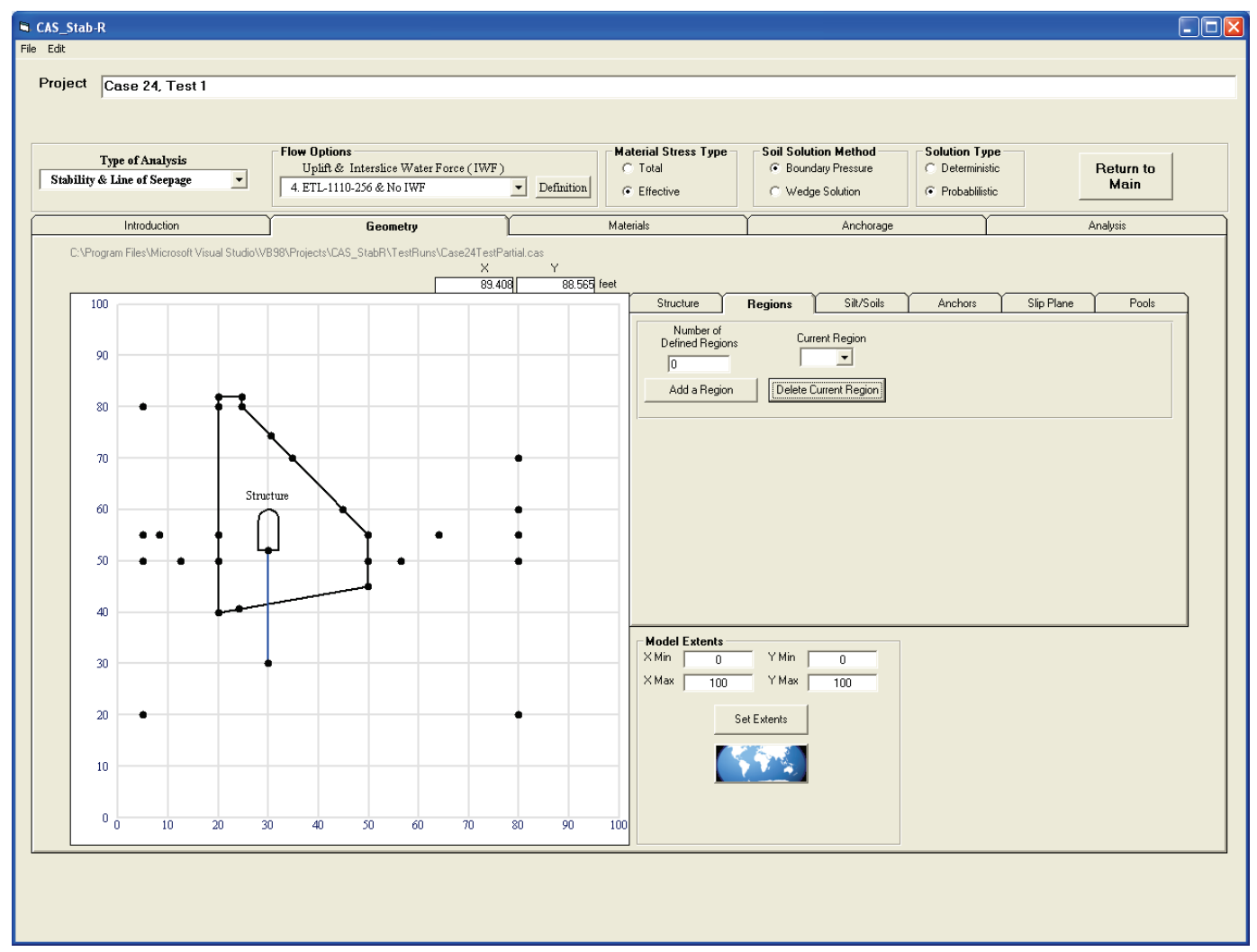

Figure 3-32. Instructions for defining a material region.

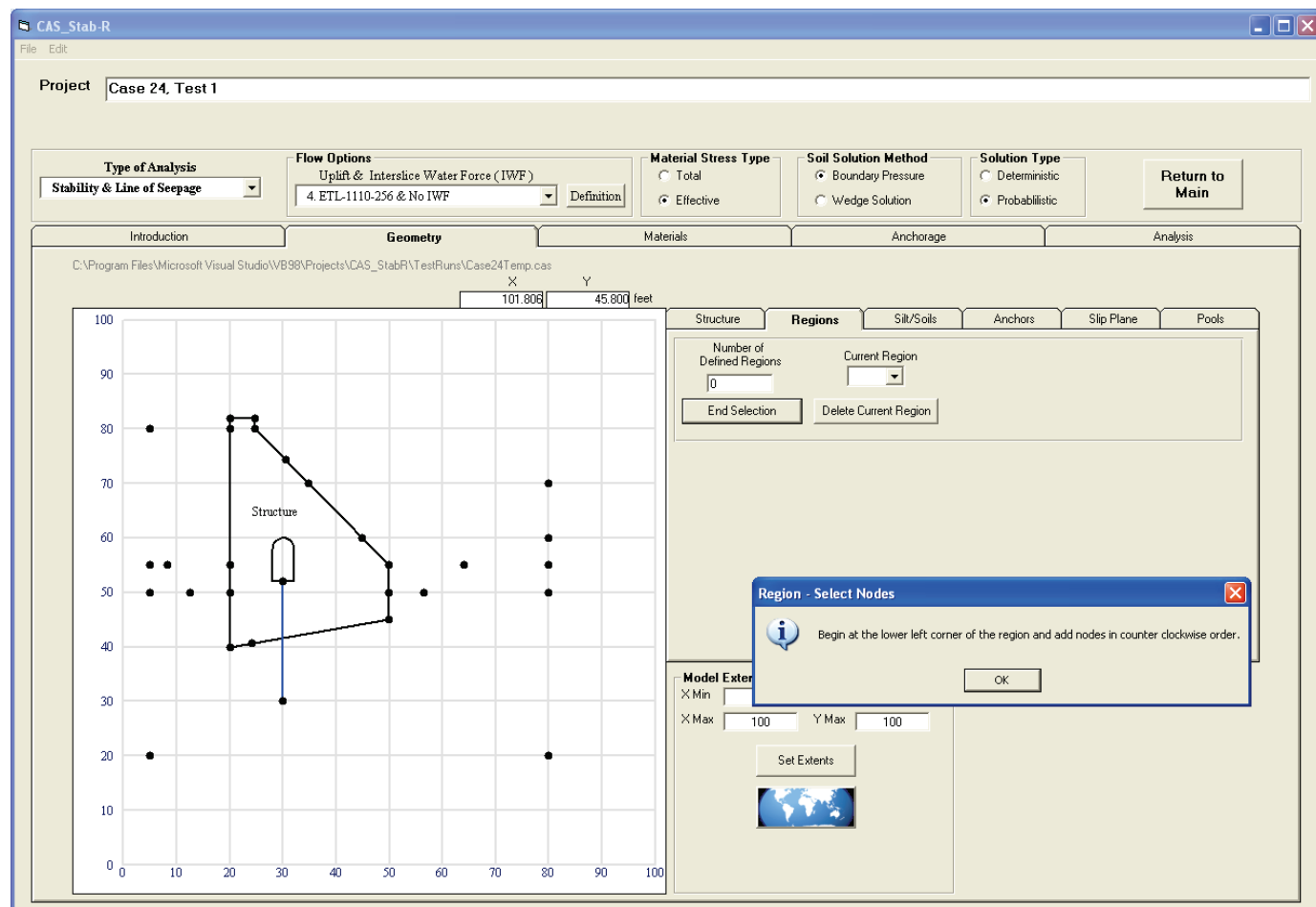


Highlight nodes with the cursor to define the region, beginning with the lower left corner of the region. Click the highlighted node with the left mouse button. A message appears in the Message Area to indicate the node was added to the region. Add nodes in counterclockwise order until all nodes have been selected. As nodes are selected, line segments are added to the display. An orange line is added between the last node selected and the first node so that the region shape can be observed as it would be if the selection process were ended at that point. An example of a partially completed region is shown in Figure 3-33. After selecting all the nodes that define the region, click the End Selection button (it is in the same location as the Add a Material Region button). The completed region will be added to the display. The Geometry Display with a completed material region is shown in Figure 3-34. This region consists of nodes 18, $19,17,16,11,10,15$, and 14 specified in the order listed.

To the right of the Number of Defined Regions input box is a drop-down list box labeled Current Region. This list box is used to select a region for deletion from the model. To delete a region, select the desired region with the list box as shown in Figure 3-35 and click the Delete Current Region button. The region will be removed from the model.

Figure 3-33. Geometry Display with a partially defined region.

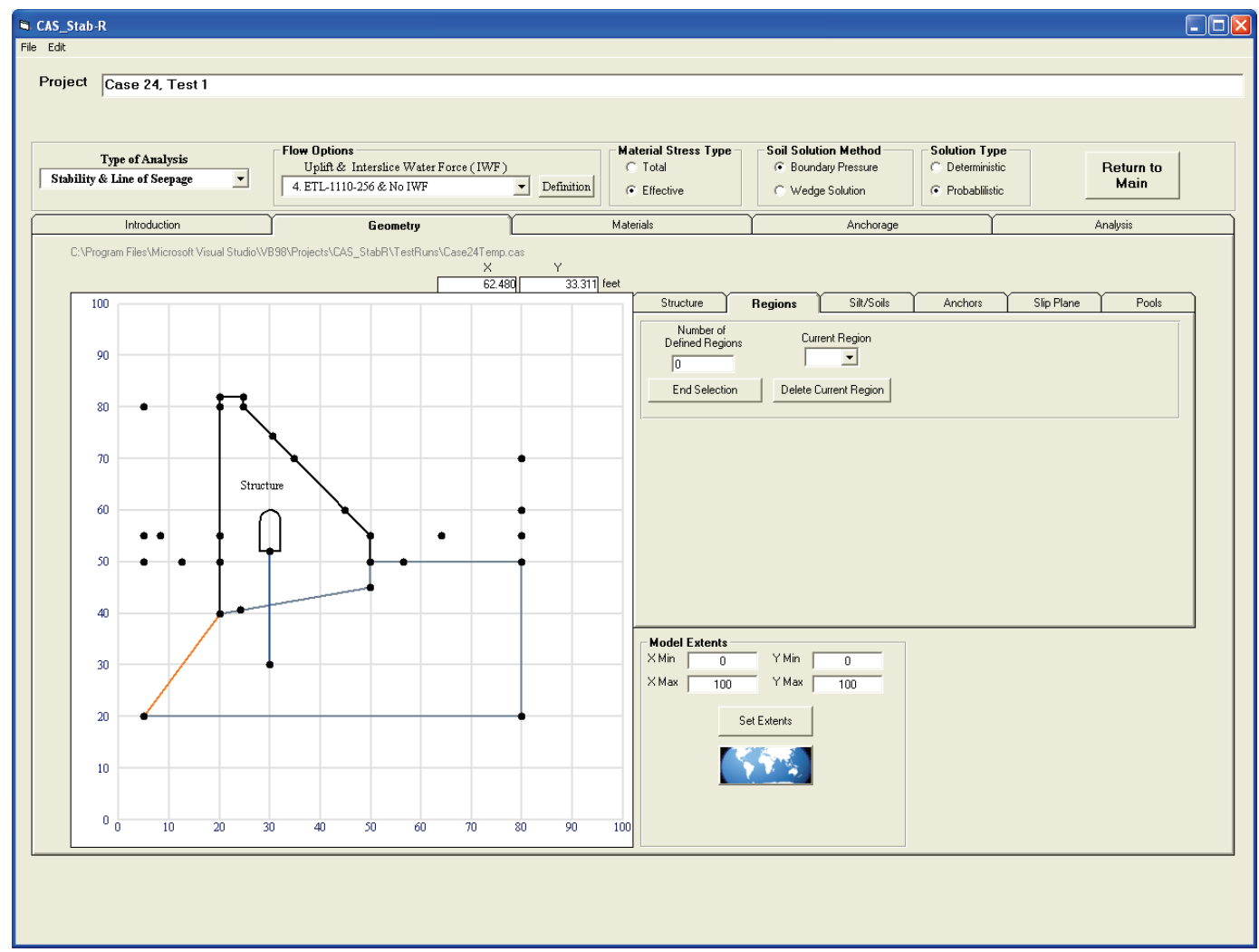


Figure 3-34. Geometry Display with a completed material region.

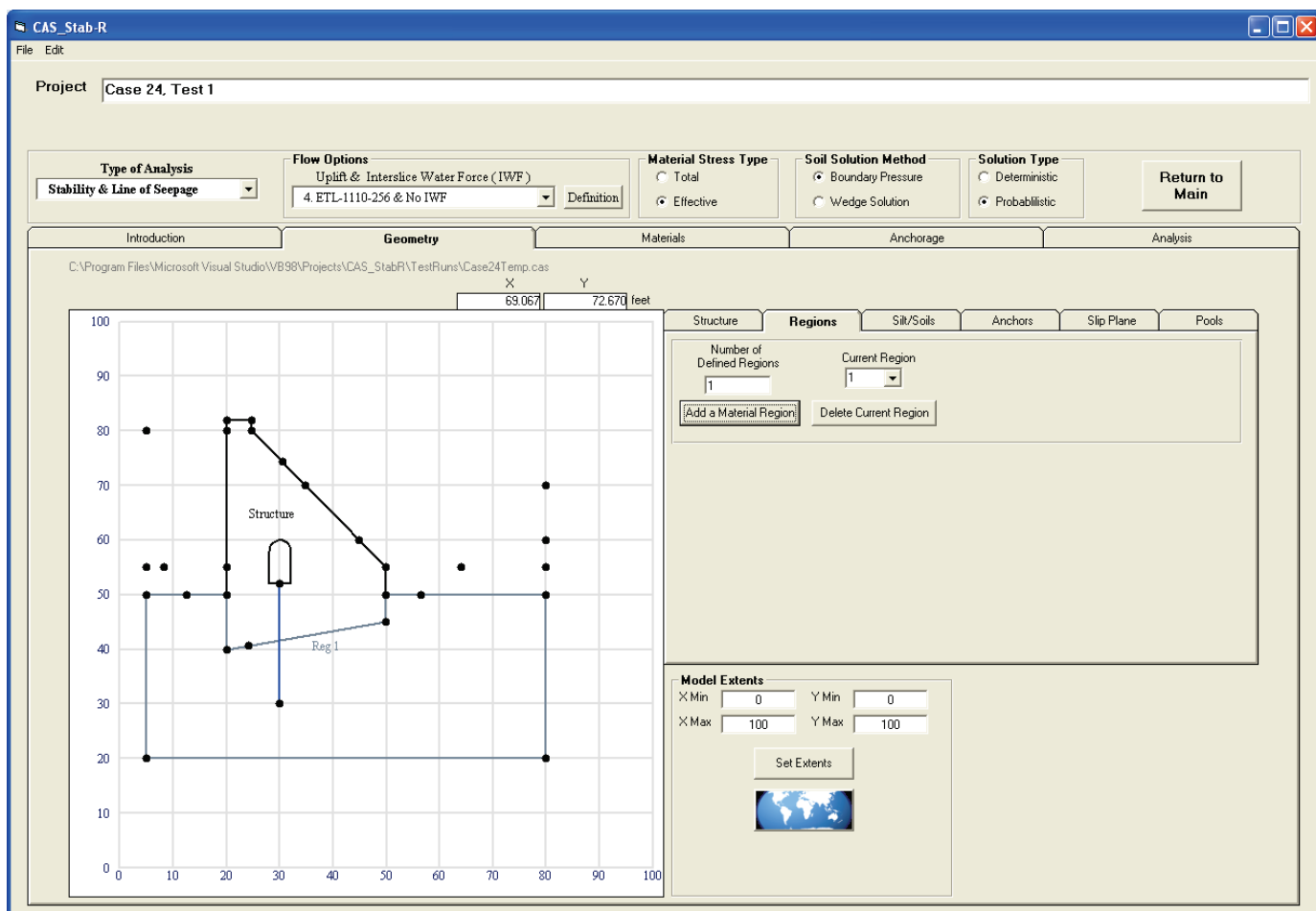

Figure 3-35. Regions tab with the Current Region list box highlighted.

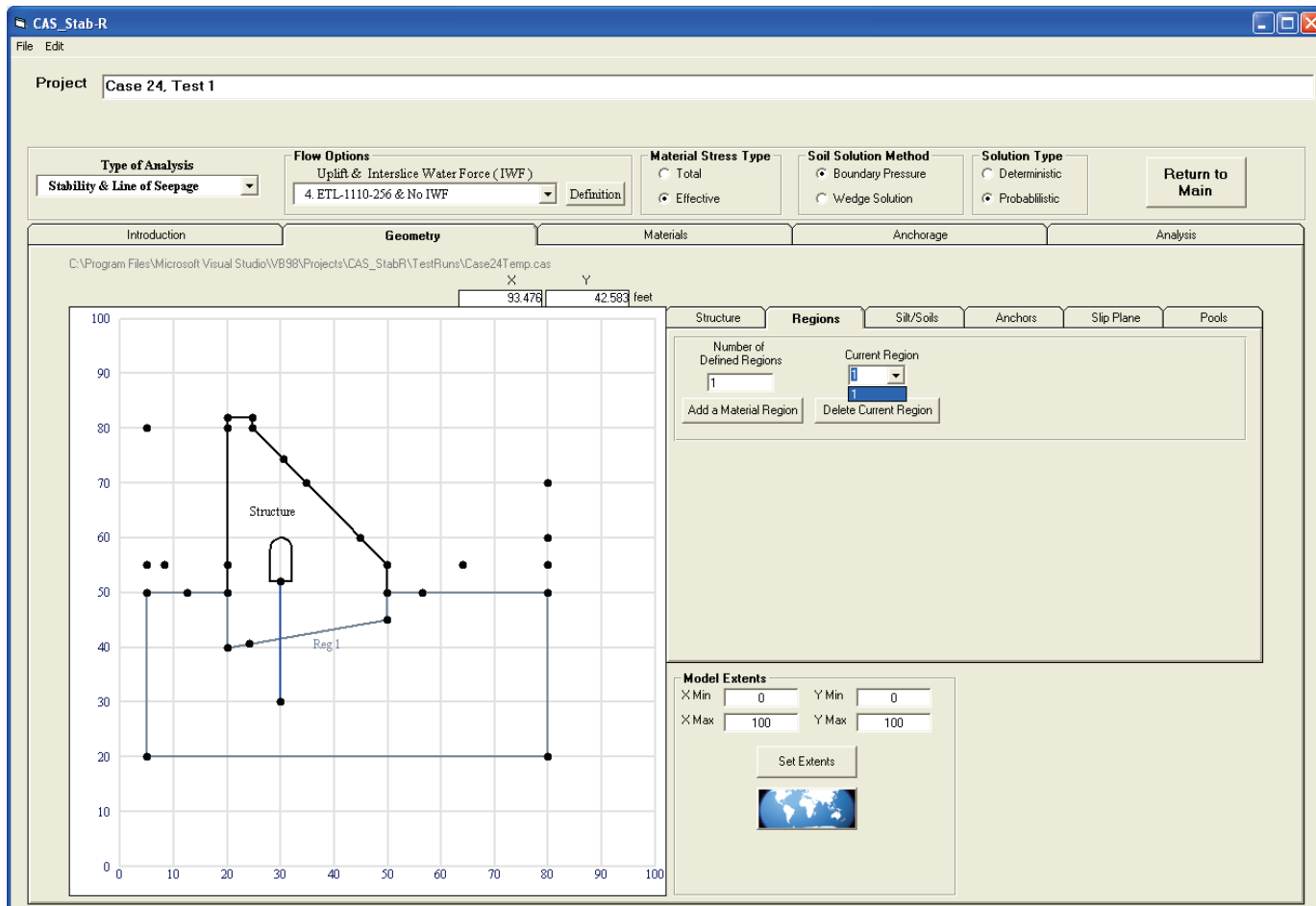




\subsubsection{Silt/Soils tab}

Silt and/or soil layers may be added utilizing the Silt/Soils tab to the right of the Geometry Display. This tab is shown in Figure 3-36. To add a silt layer to the model, make sure the Use Silt Layer check box is checked and supply the elevation of the silt layer, the density of moistened silt, the density of saturated silt, and the horizontal earth pressure coefficient in the appropriate text boxes to the right of the Silt Level label. Note that the top of the silt is indicated in the Geometry Display as a sand-colored line. The silt layer may be removed from the model by clicking the Use Silt Layer check box so that it is cleared.

Figure 3-36. Silt/Soils tab with a defined silt layer at elevation $60 \mathrm{ft}$.

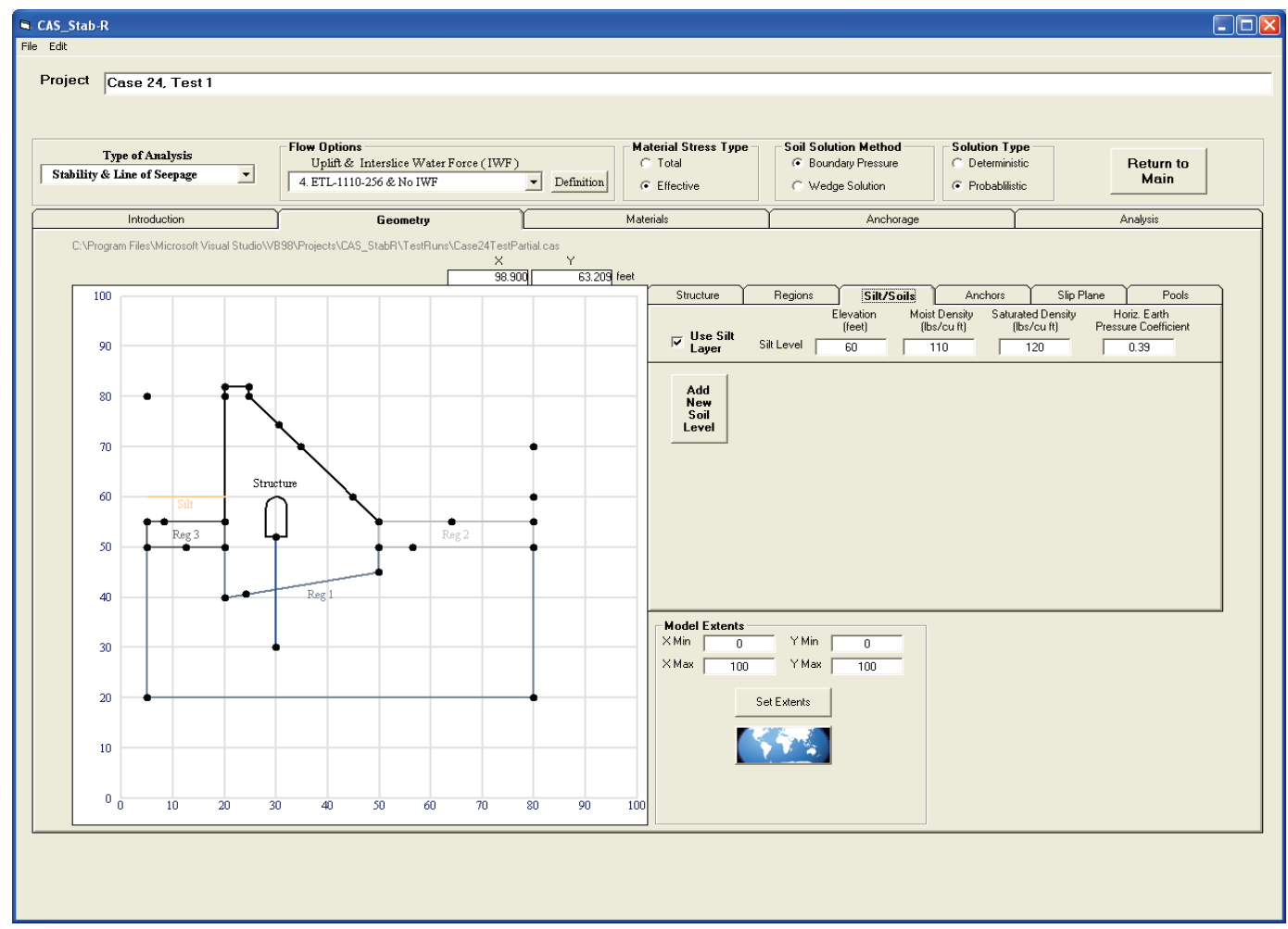

A soil layer is added by clicking the Add New Soil Level button located beneath the dividing line on the Silt/Soils tab. Clicking this button adds input boxes for the soil elevation (default $=-1000000000$ ), the density of the moistened soil (default $=120$ ), the density of the saturated soil (default $=125$ ), and the horizontal earth pressure coefficient (default $=0.5$ ) of the soil as shown in Figure 3-37. The user may edit the parameters as needed for this soil layer. Up to 10 soil layers may be defined. To remove a defined soil layer from the model, click the button labeled " $x$ " to the right of the soil layer parameters. Figure 3-38 shows a display with two soil layers defined. 
Figure 3-37. Silt/Soils tab after clicking Add New Soil Layer.

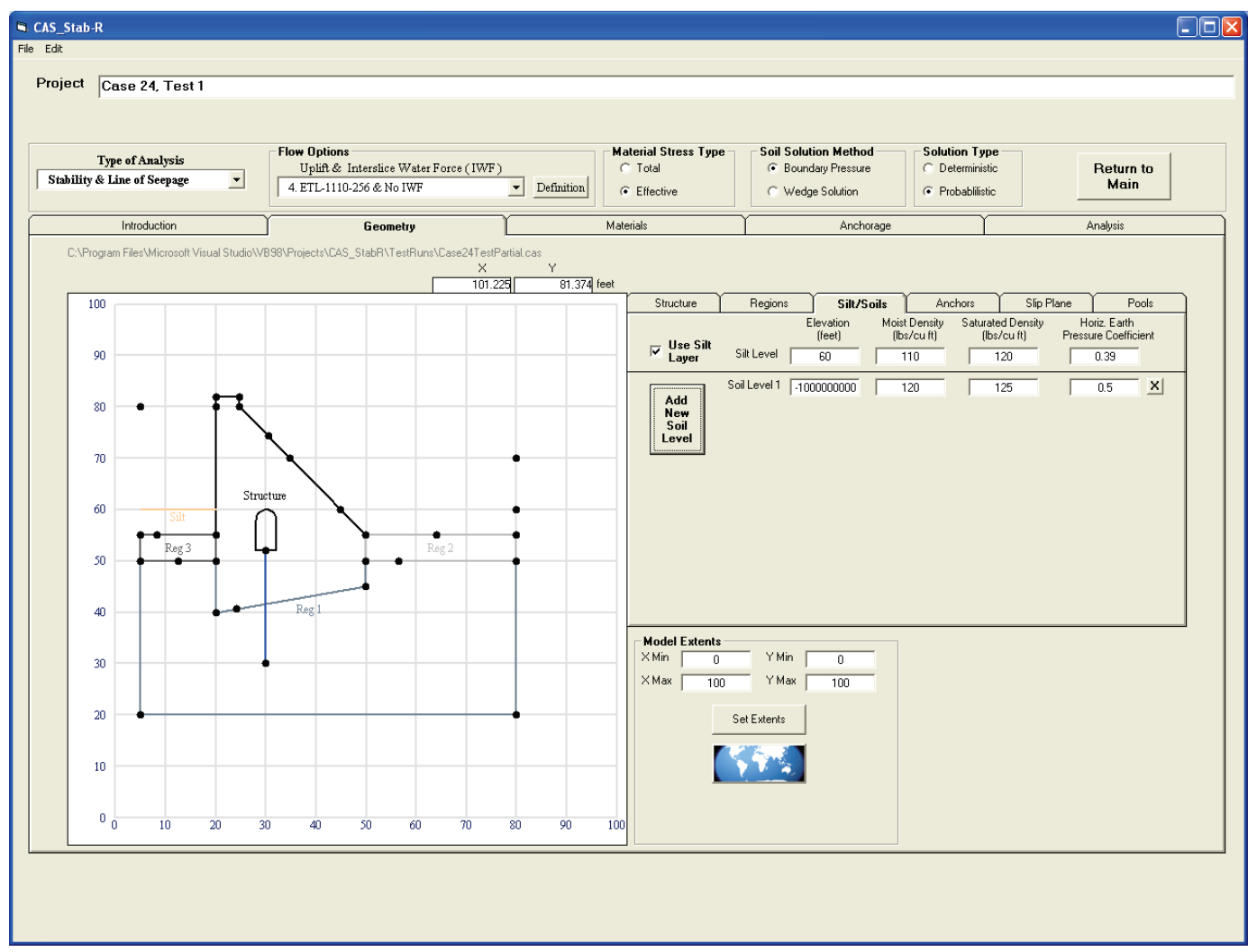

Figure 3-38. Model with Soil Layers defined at elevations 60 and $70 \mathrm{ft}$.

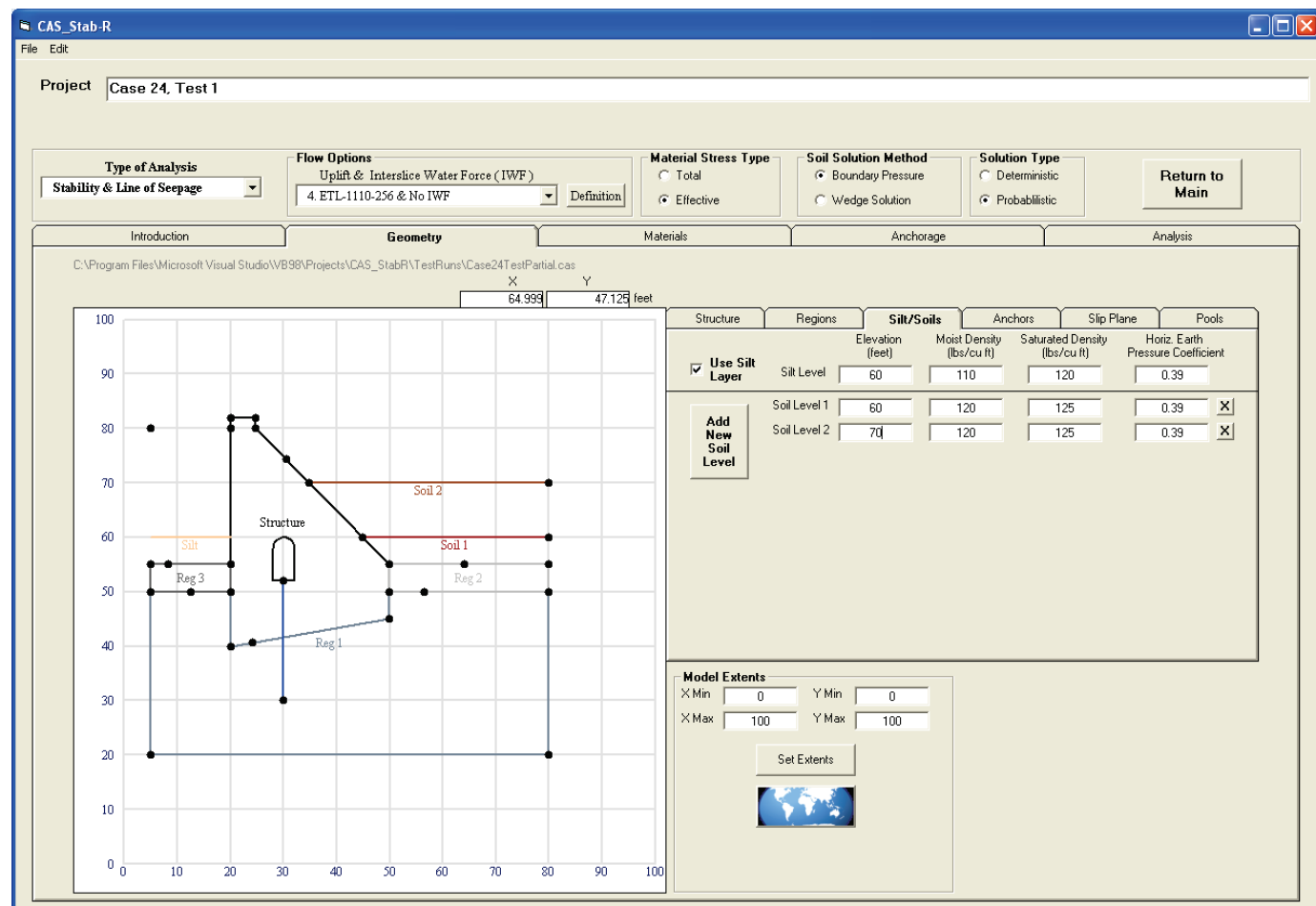




\subsubsection{Anchors tab}

Anchor locations in the model are specified utilizing the Anchors tab to the right of the Geometry Display. Clicking the Anchors tab results in the window shown in Figure 3-39. To add an anchor to the model, click the Add an Anchor button. Two new input boxes will appear below the Node Top of Anchor and Node - Bottom of Anchor labels. Three buttons are also added. One button, Select Top Node, is used to select the node as the location of the anchor top. Another button, Select Bottom Node, is used to select the node as the location of the anchor bottom. The third button is labeled " $x$ " and is used to delete an anchor from the model. The new controls are shown in Figure 3-40.

To select the top of the anchor, click the Select Top Node button. An information window will appear as shown in Figure 3-41 with instructions to follow to make the selection. Position the cursor over the node at the top of anchor location to highlight the node. Left-click the mouse, and the node number will populate the Node - Top of Anchor input box.

Figure 3-39. Initial Geometry-Anchors tab.

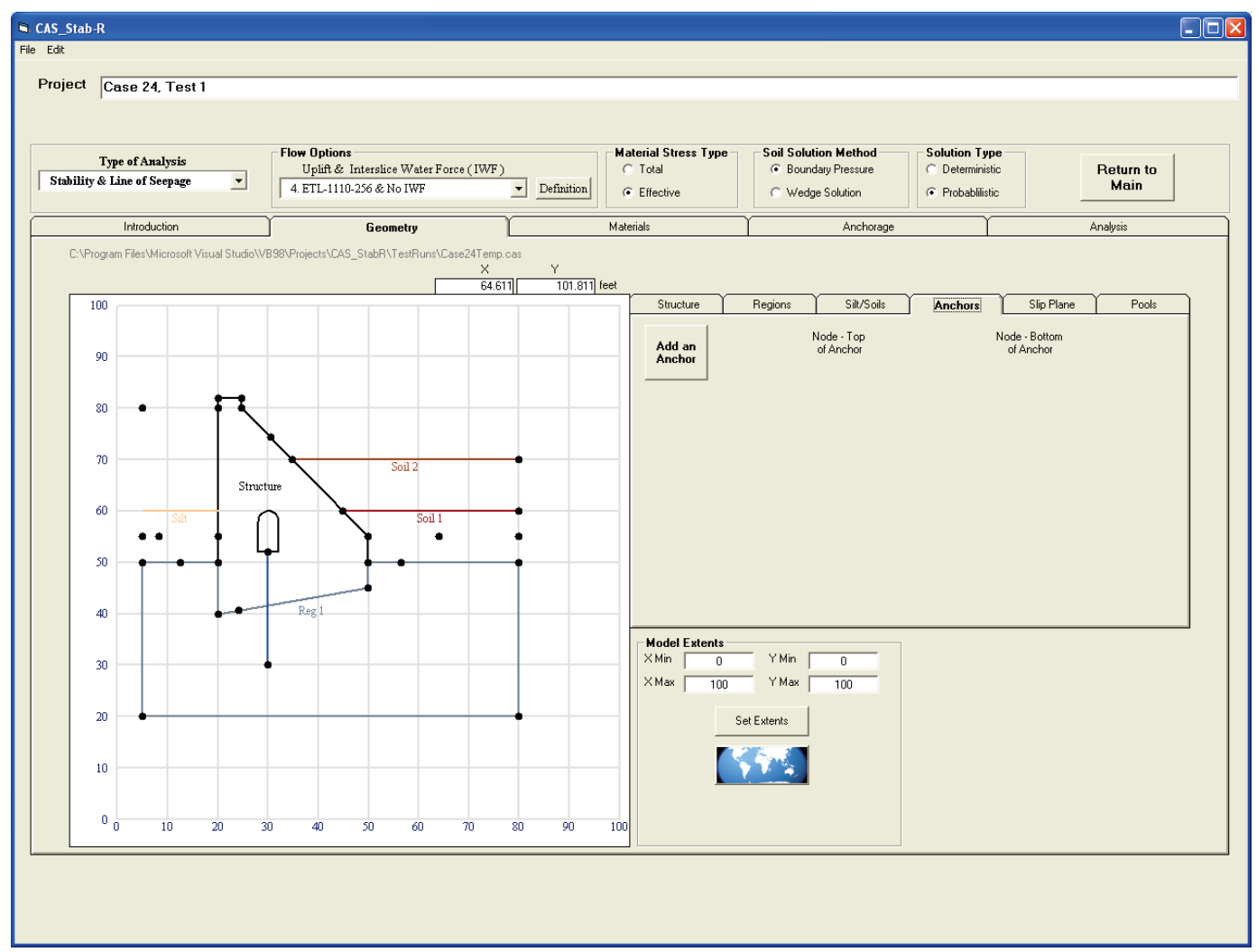


Figure 3-40. Geometry-Anchors tab after clicking Add an Anchor button.

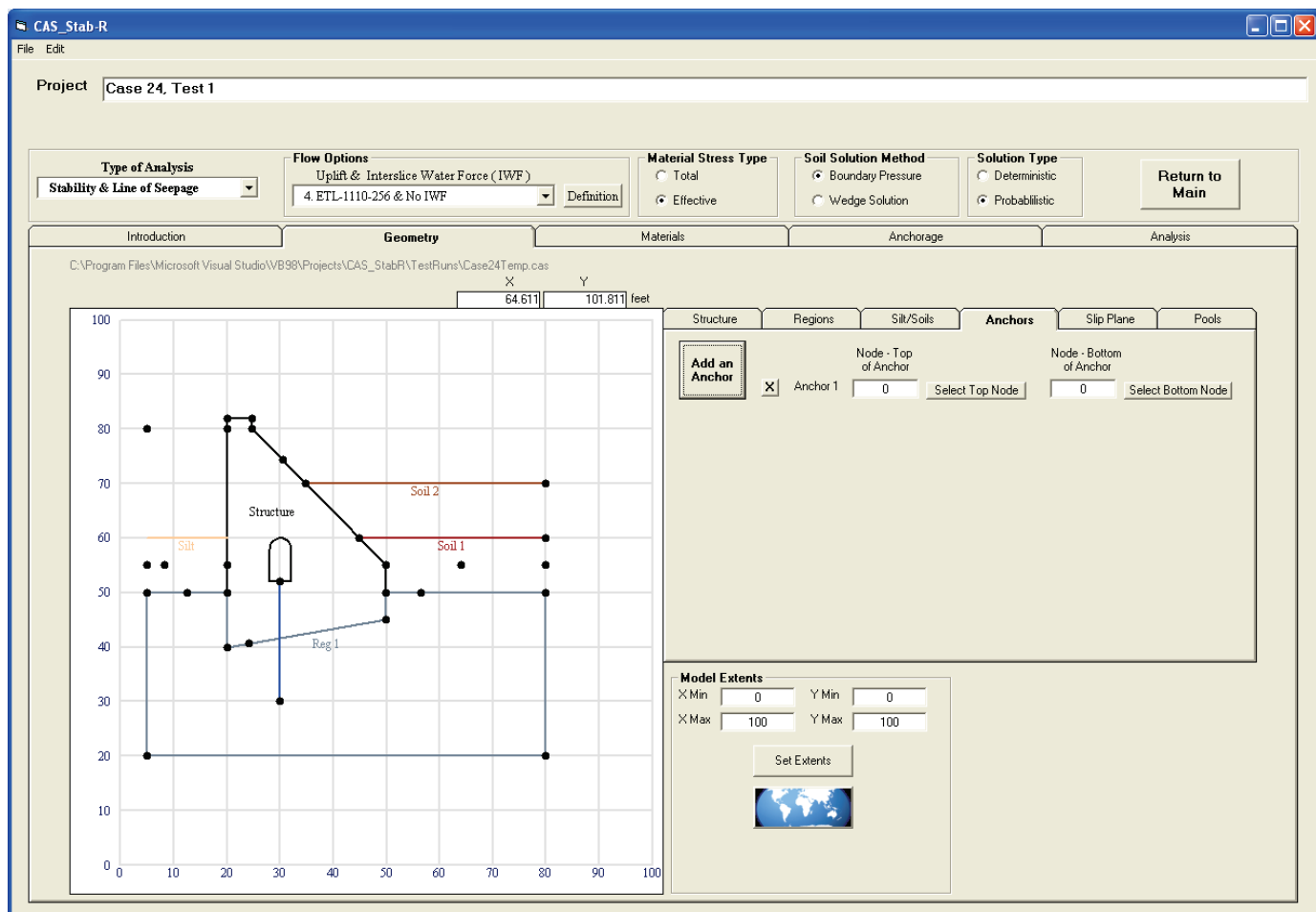

Figure 3-41. Instruction window to specify the Anchor top node.

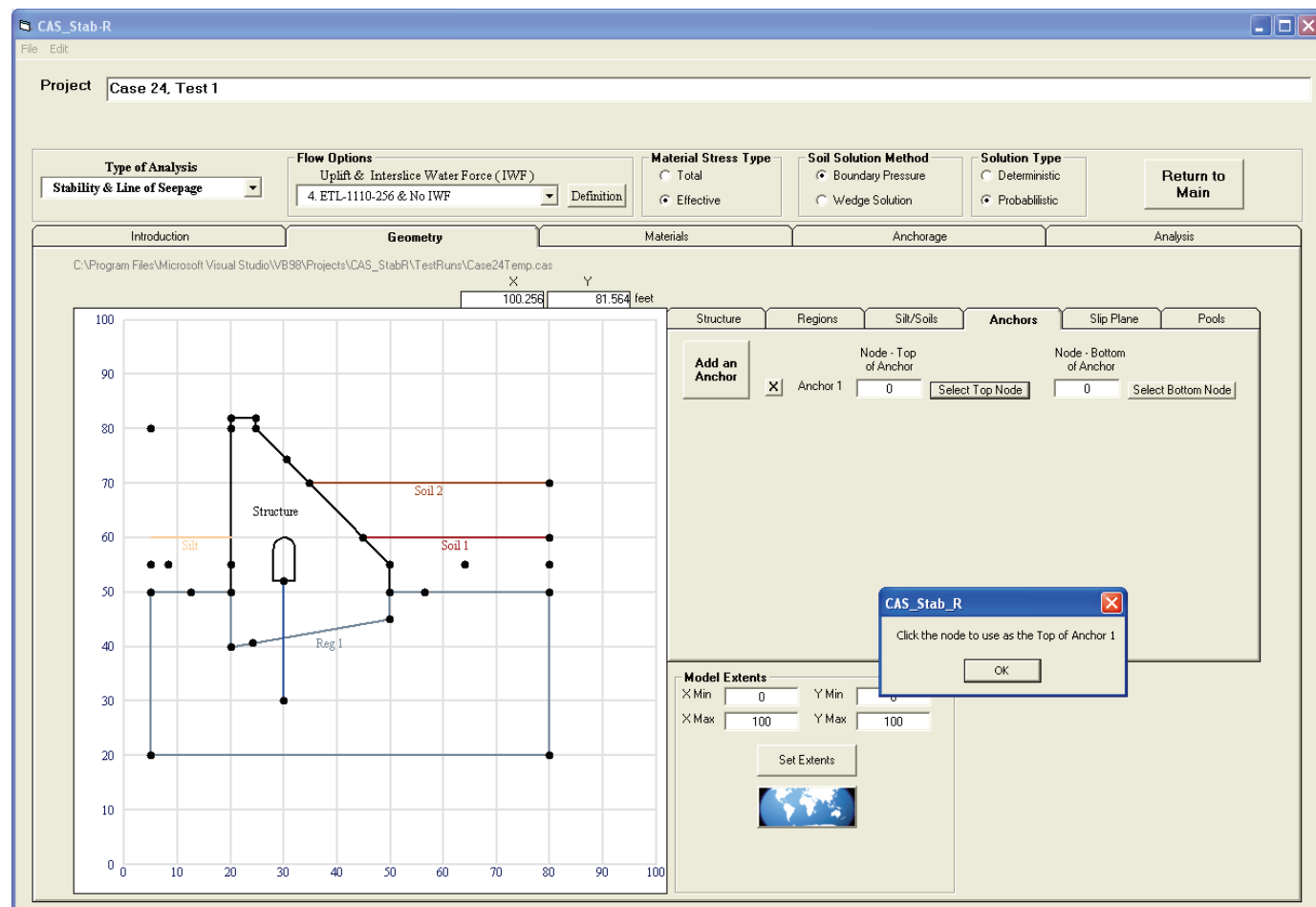


To select the bottom of the anchor, click the Select Bottom Node button. The instructions are the same as for the selecting the top node as discussed in the previous paragraph. Following the selection of the top and bottom nodes of the anchor, the anchor will display in the color green in the Geometry Display as shown in Figure 3-42.

Figure 3-42. Geometry Display with an anchor shown in green.

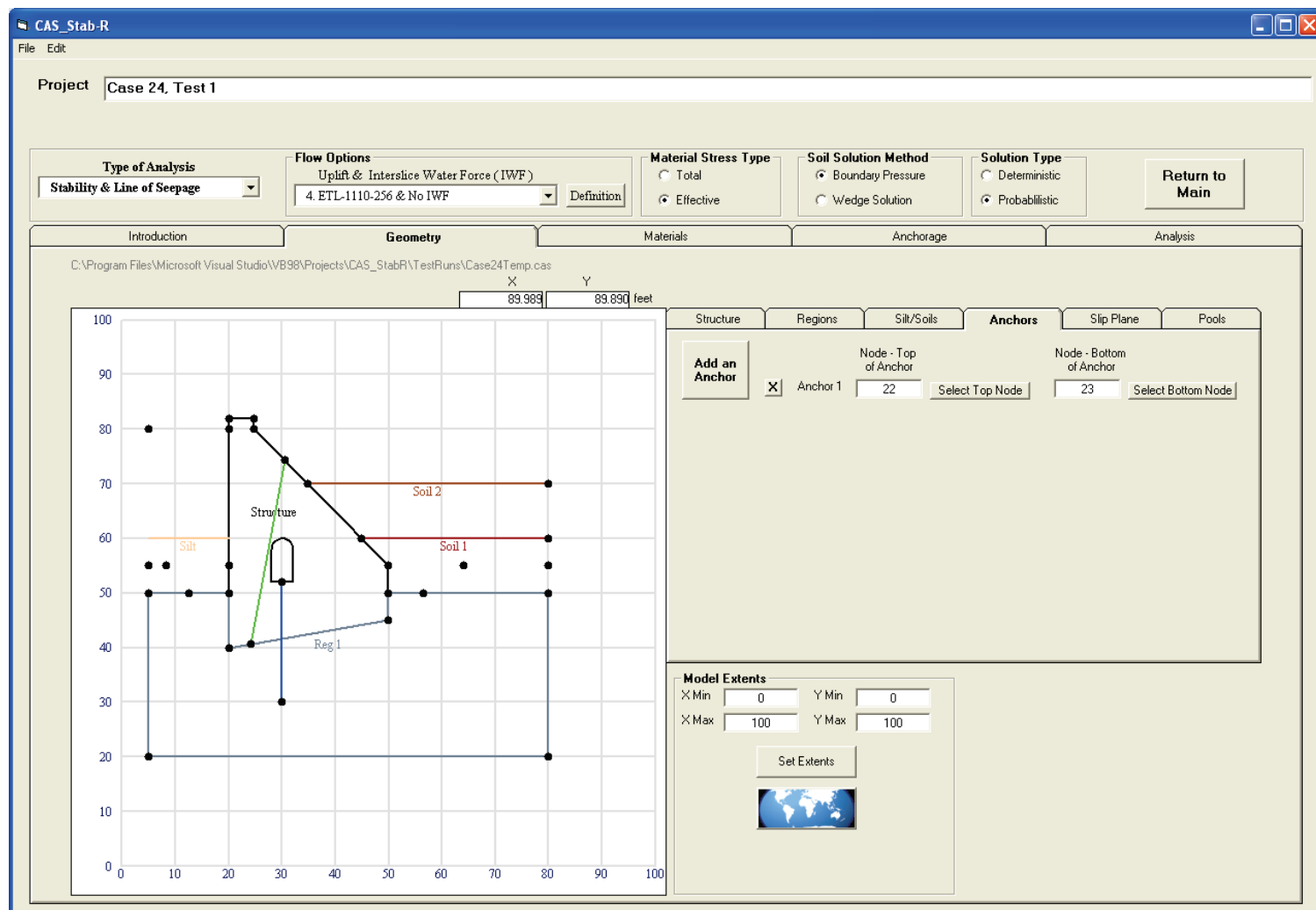

The nodes for the top and the bottom of the anchor may also be specified by directly entering the correct node numbers into the Node - Top of Anchor and Node - Bottom of Anchor input boxes.

Additional anchors may be added by clicking the Add an Anchor button and following the previously described procedure. The model may contain a maximum of 10 anchors. An anchor may be removed from the model by clicking the " $x$ " button immediately to the left of the anchor to be removed. 


\subsubsection{Slip Plane tab}

The potential Slip Plane for the structure is defined using the Slip Plane tab to the right of the Geometry Display. This tab contains a button labeled Select Slip Plane Nodes as shown in Figure 3-43. Clicking this button produces the instruction window shown in Figure 3-44. Click the OK button. CAS_Stab-R is now in Select Slip Plane Nodes mode, and the tab now contains a button labeled End Selection. Select the nodes for the Slip Plane by positioning the cursor over the left-most node of the desired Slip Plane so that node is highlighted. Click on the node with the left mouse button. A message indicating the node has been added to the Slip Plane is shown in the Message Area while the mouse button is held down as shown in Figure 3-45. Continue adding nodes moving from left to right until the final node has been selected. As nodes are added, red line segments are added to the Geometry Display to indicate the current state of the Slip Plane definition. After the final node has been added, click the End Selection button on the Slip Plane tab. Select Slip Plane Nodes mode is closed, and the Slip Plane is displayed as a red line as seen in Figure 3-46.

Figure 3-43. Slip Plane tab.

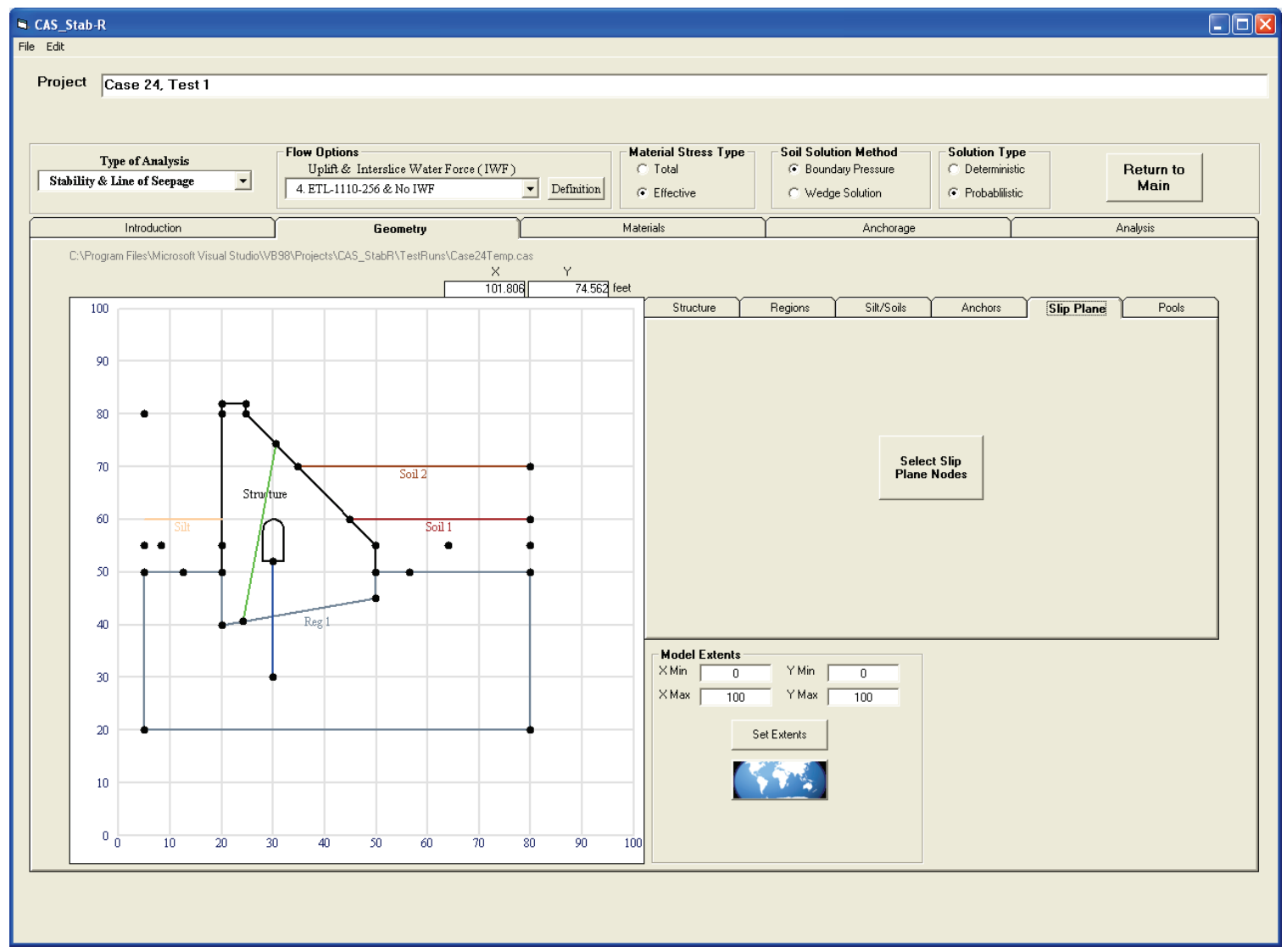


Figure 3-44. Instructions for selecting slip plane nodes.

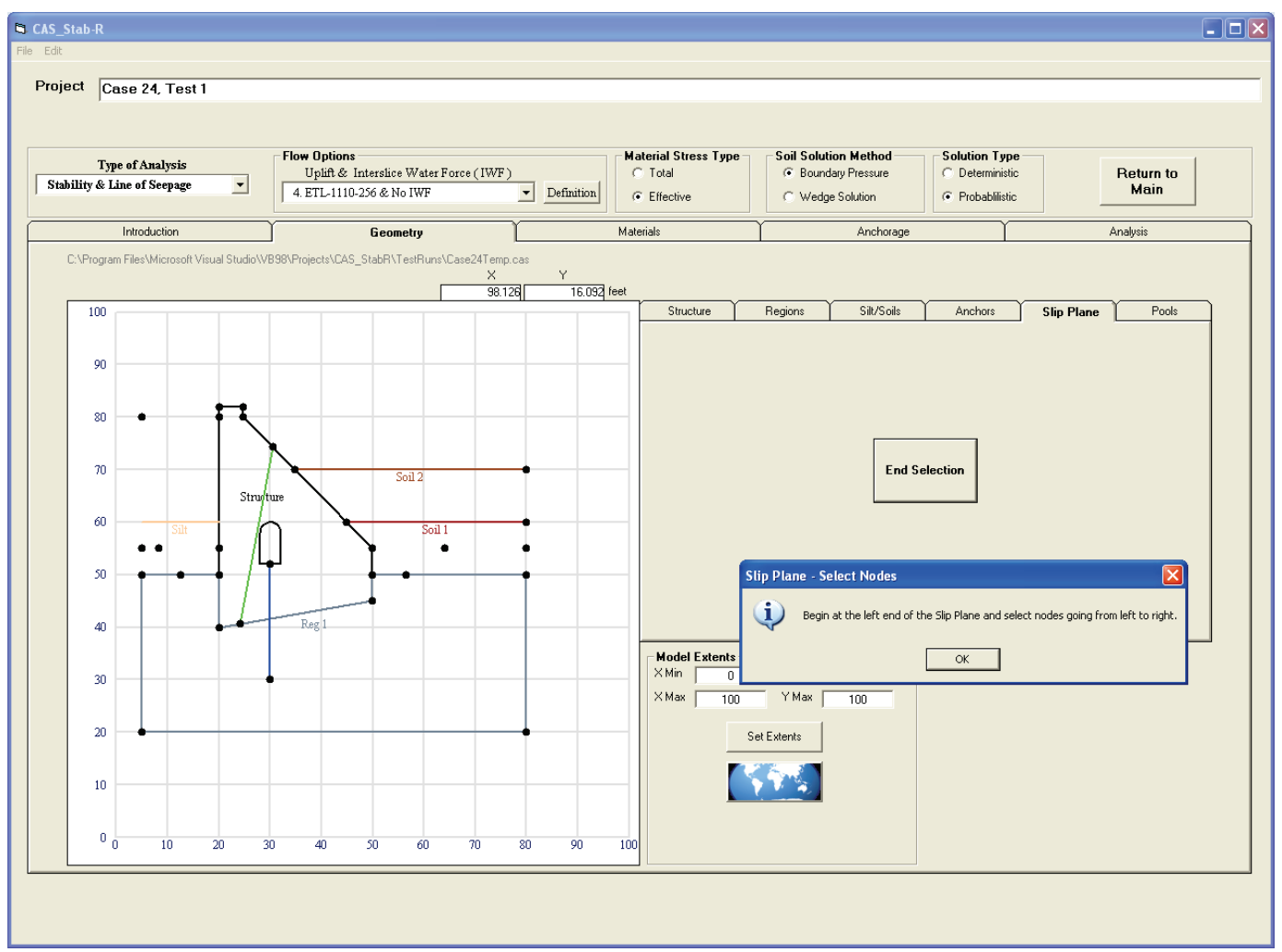

Figure 3-45. Partially defined slip plane.

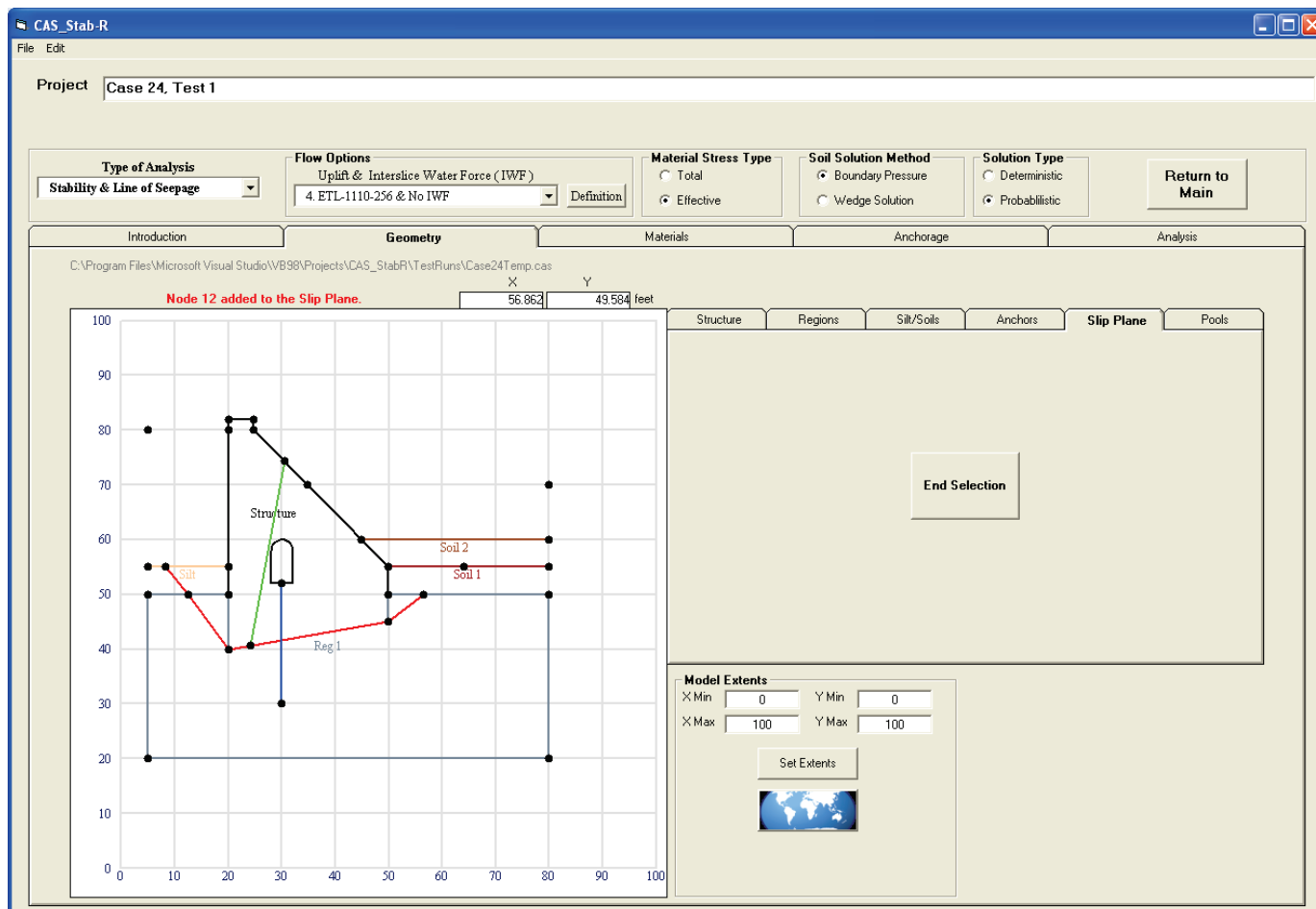


Figure 3-46. Completed potential slip plane.

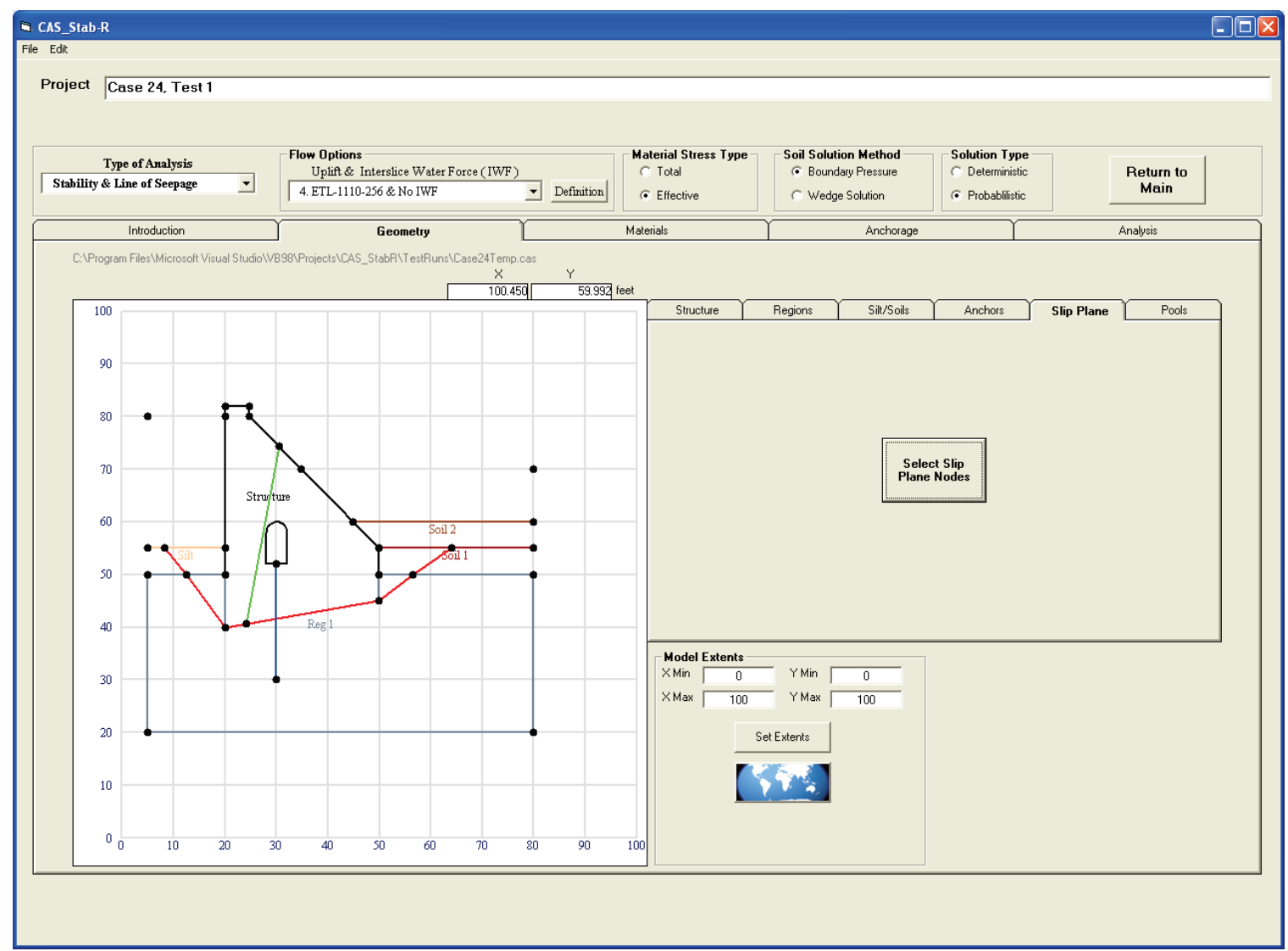

\subsubsection{Pools tab}

The Pools tab to the right of the Geometry Display provides the means to specify the headwater elevation, tailwater elevation, and the value for the density of water that will be used in the analysis. The Pools tab is shown in Figure 3-47.

A blue line in the Geometry Display will be shown at the specified elevation on the headwater side of the structure after a valid headwater elevation is entered into the Headwater Elevation input box. Likewise, a valid value in the Tailwater Elevation input box will produce a blue line at the specified tailwater elevation on the tailwater side of the structure in the Geometry Display. Figure 3-48 shows the display with headwater elevation of $80 \mathrm{ft}$ and tailwater elevation of $70 \mathrm{ft}$. While not a part of the Geometry Display, the value for the density of water has been set to 62.4 pounds per cubic foot (lb/ft3) in Figure 3-48. 
Figure 3-47. Geometry-Pools tab.

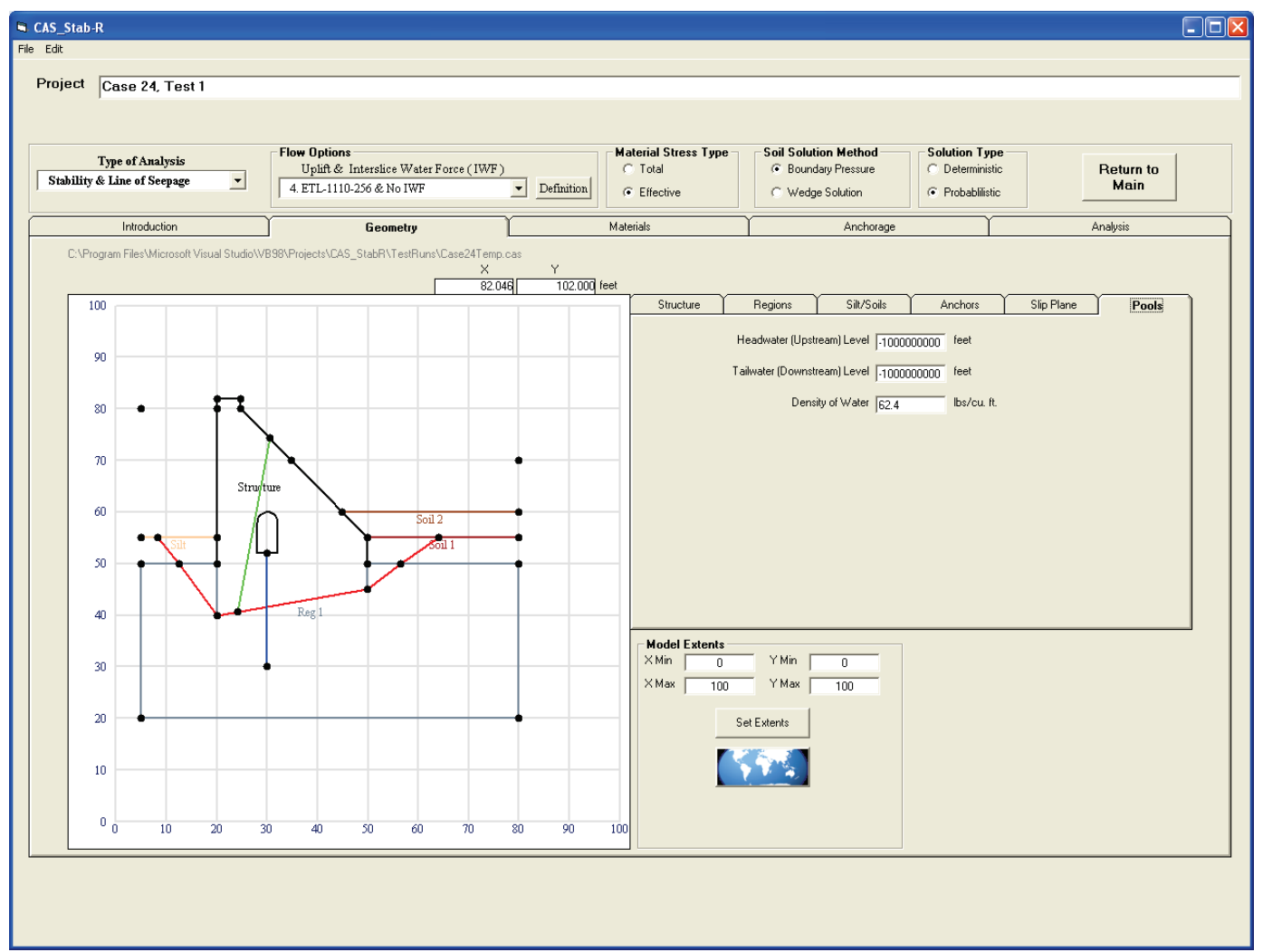

Figure 3-48. Resulting display with Headwater Level $=80 \mathrm{ft}$ and Tailwater Level $=70 \mathrm{ft}$.

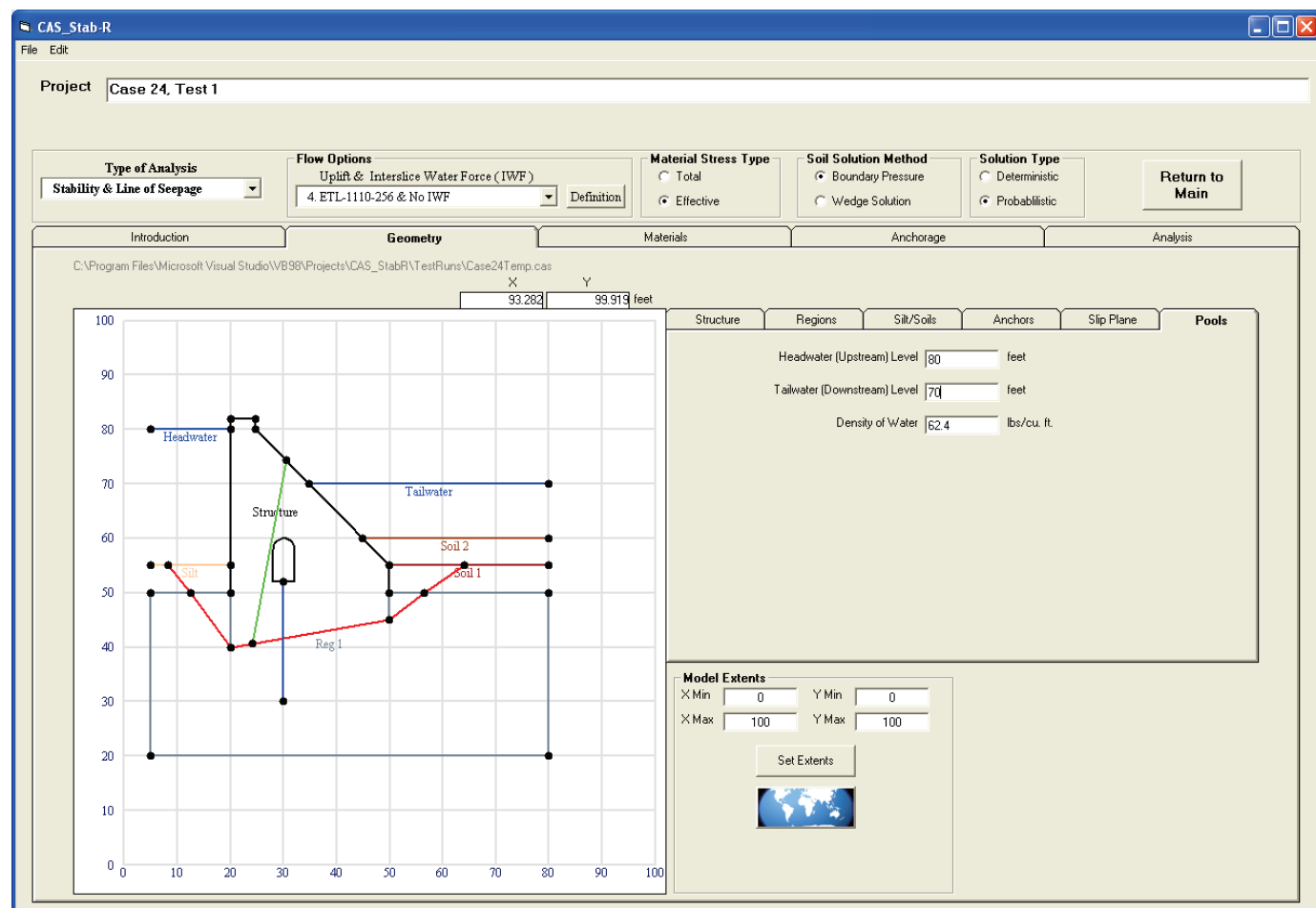




\subsubsection{Materials tab}

The user provides the properties of the materials that comprise the foundation regions and the structure. The initial Materials tab is shown in Figure 3-49. This tab contains two sections for user input. The section labeled Material Properties allows the user to add materials and set the material properties for each. The section labeled Region - Material Mapping allows the user to specify which material numbers correspond to the regions defined on the Geometry Regions tab and which material corresponds to the structure.

Figure 3-49. Initial Materials tab.

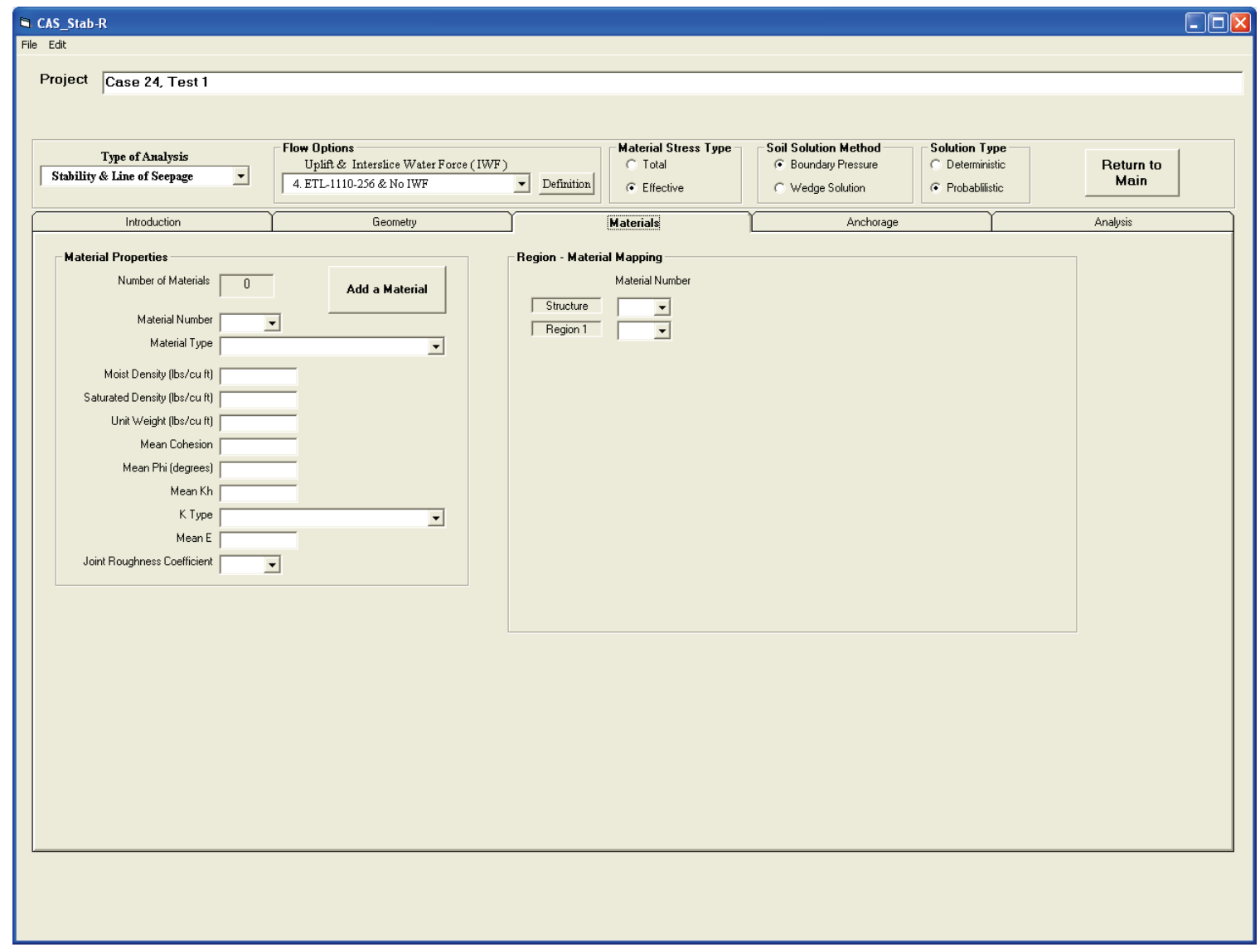

\subsubsection{Material Properties section}

Materials are added and assigned property values in this section of the Materials tab. Click the Add a Material button, and the display will appear as shown in Figure 3-50. The components of this section are discussed in the following sections. 
Figure 3-50. Result of clicking the Add a Material button.

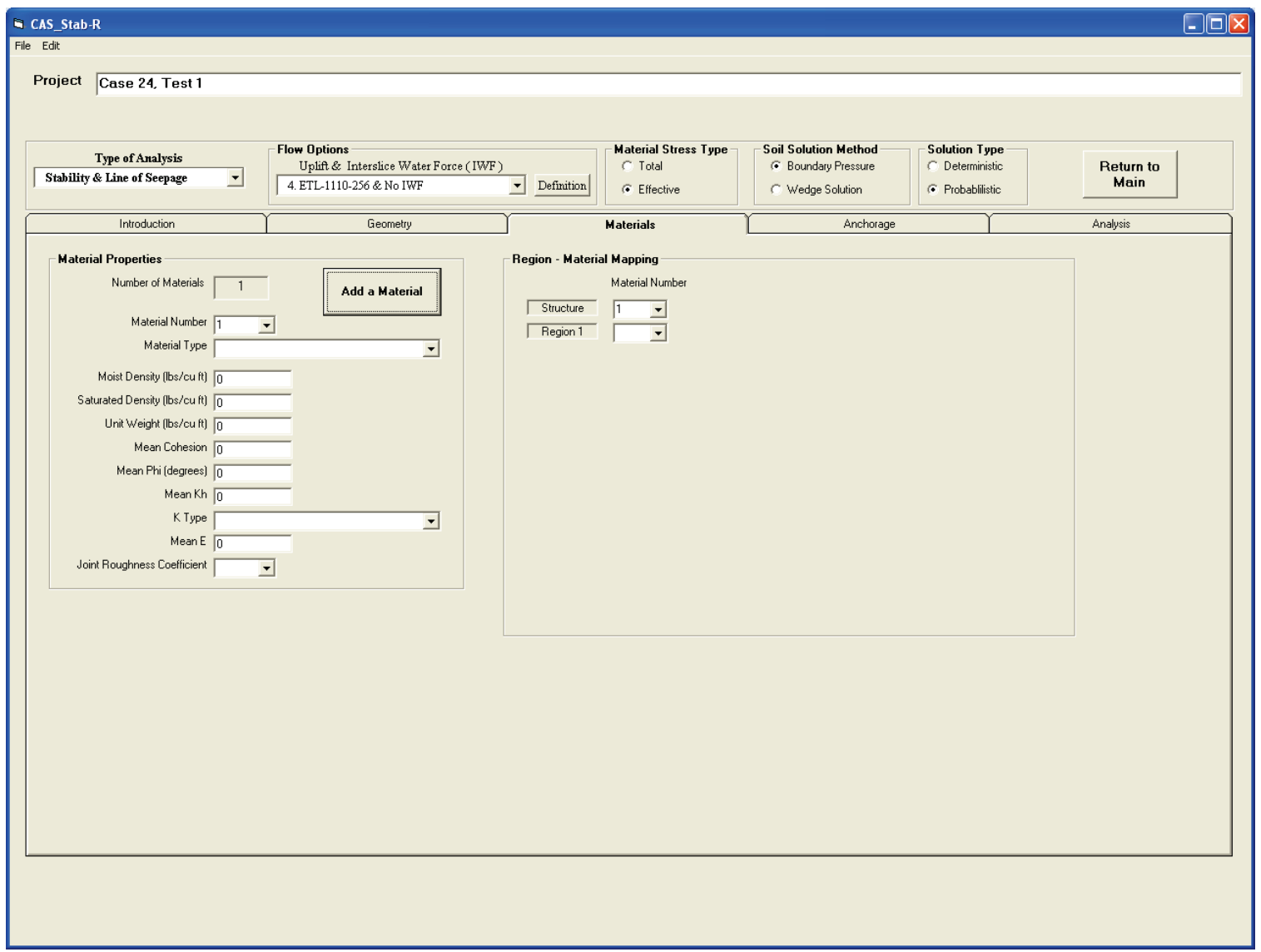

The new Material Number is automatically assigned to be the number of existing materials plus 1 . In this case no other materials had previously been defined, so the Material Number is assigned to be 1. The Material Number is a drop-down box used to select any defined material and observe and alter the property values assigned to it. The properties of a material and the means to assign values to it are discussed in the following sections.

\section{Material Type property}

The material type can be one of three values and is selected using the drop-down box labeled Material Type as shown in Figure 3-51. The three material types are Rock, Concrete, and Soil. The material type chosen should be Rock for the rock foundation regions, Concrete for the structure, or Soil for a soil region. Soil regions need be defined only when the Soil Solution Method is selected as the Wedge Solution. The Soil Solution Method is discussed elsewhere in this report. 
Figure 3-51. Material type drop-down box.

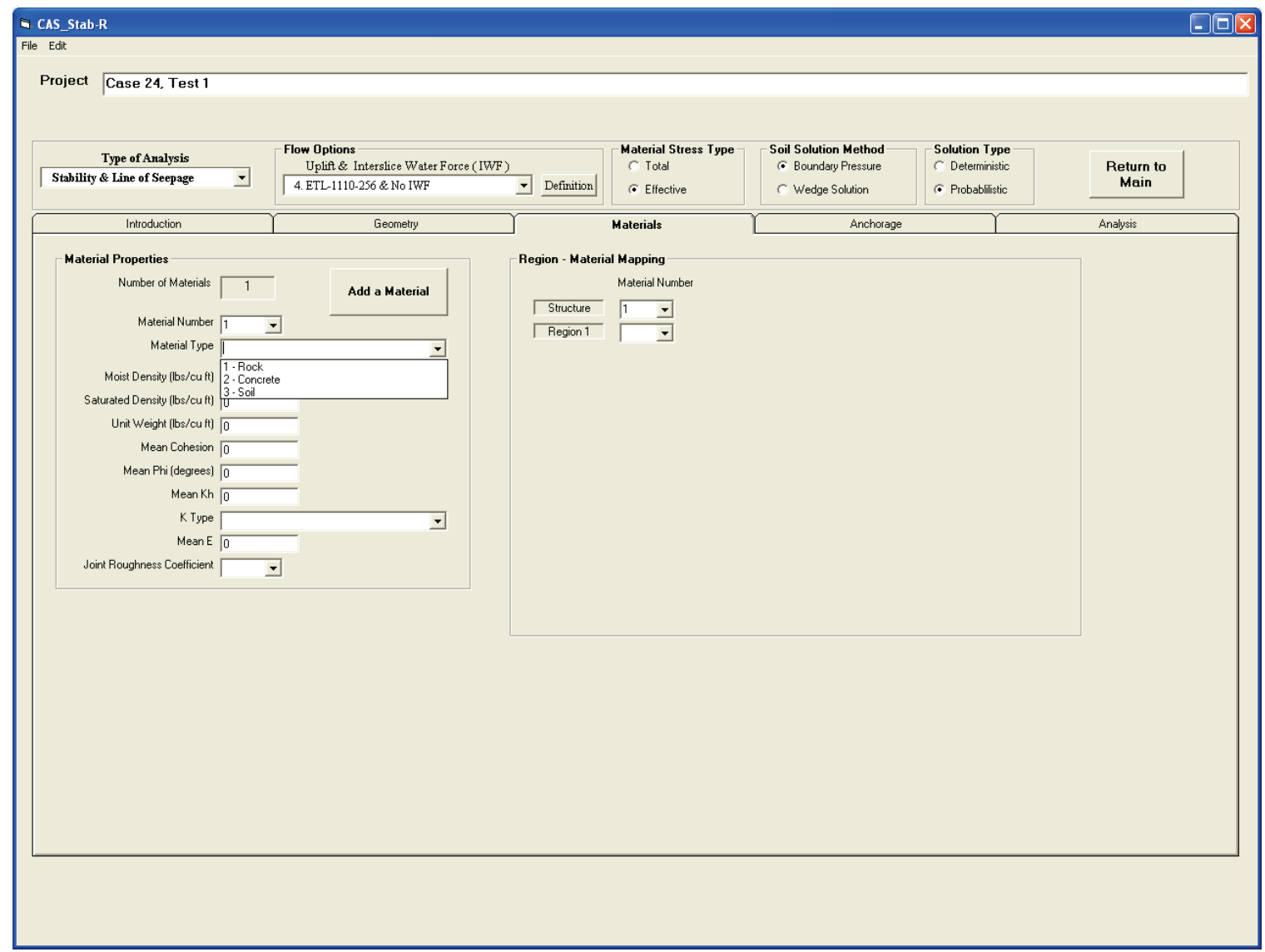

\section{Moist Density property}

The moist density of the material is specified by entering the value into the text box labeled Moist Density. This property is valid for rock or soil materials.

\section{Saturated Density property}

The saturated density of the material is specified by entering the value into the text box labeled Saturated Density. This property is valid for rock or soil materials.

\section{Unit Weight property}

The unit weight of the material is specified by entering the value into the text box labeled Unit Weight. This property is valid for concrete materials. 


\section{Mean Cohesion property}

The mean value for the cohesion of the material is specified by entering the value into the text box labeled Mean Cohesion.

\section{Mean Phi property (internal friction angle)}

The mean value of the internal friction angle, phi, is specified by entering the value into the text box labeled Mean Phi.

\section{Mean Kh property (horizontal earth pressure coefficient)}

The mean value of the horizontal pressure coefficient is specified by entering the value into the text box labeled Mean Kh.

\section{K-Type property (hydraulic conductivity)}

The K-Type property specifies the characterization of the hydraulic conductivity to be utilized in the analysis. The K-type is selected using the drop-down box labeled K-Type. The K-type property can have one of three values: Mechanical Aperture with JRC (JRC is the Joint Roughness Coefficient), Conducting Aperture, or Hydraulic Conductivity of Clean Joint as shown in Figure 3-52.

The following three material properties, K-Type, Mean E, and Joint Roughness Coefficient are not used in the calculations for either of the flow options 4, 5, and 6 that are implemented in CAS_Stab-R. Therefore, these input boxes are disabled in the current version. Discussion of these properties is included for completeness.

\section{Mean E property}

The mean value of the mechanical or actual aperture of the rock joint is specified by entering the value into the text box labeled Mean E. When English units are specified, E is in units of inches; when SI units are specified, $\mathrm{E}$ is in units of microns $(\mu \mathrm{m})$. 
Figure 3-52. K-Type drop-down box.

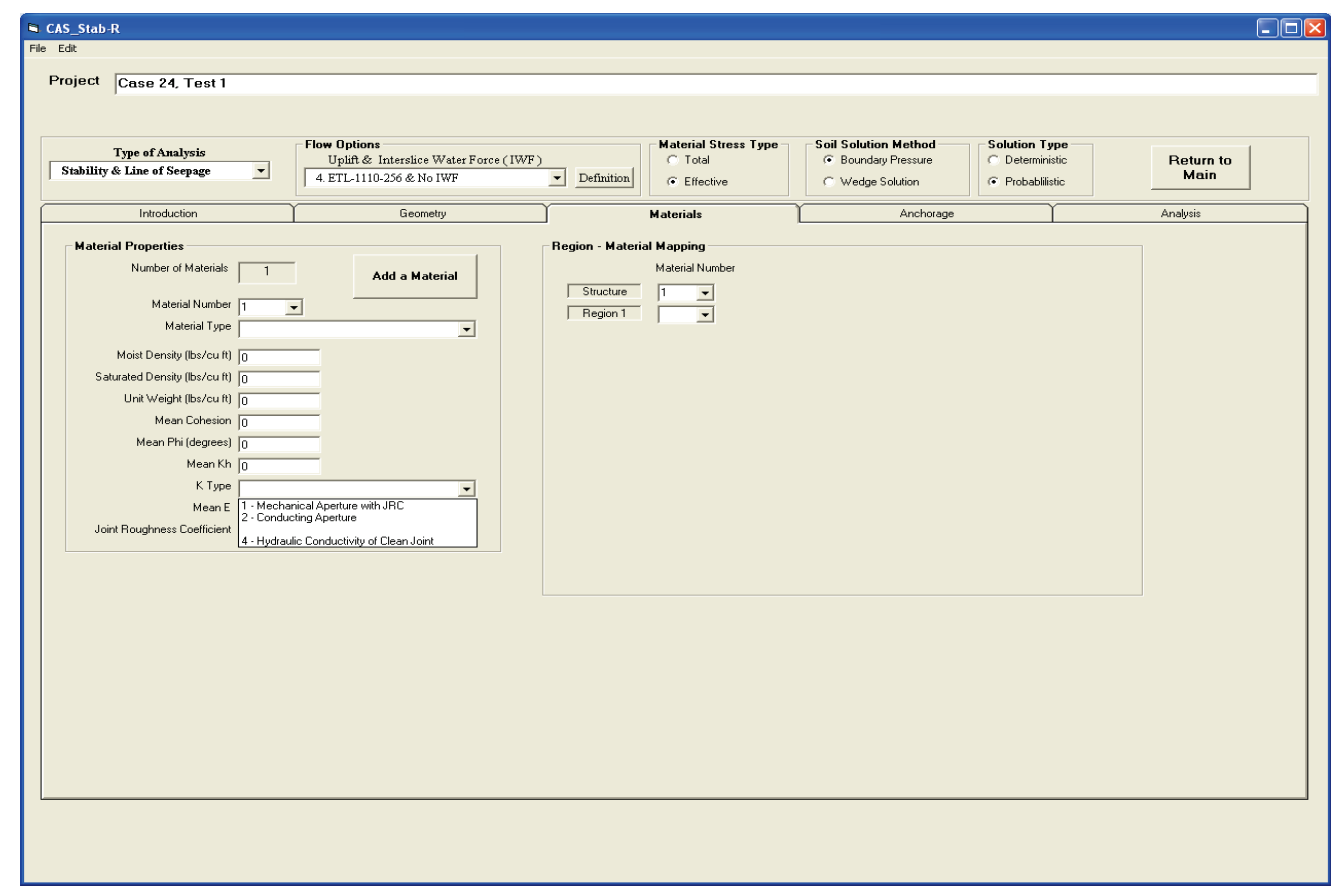

\section{Joint Roughness Coefficient (JRC)}

The JRC is specified by choosing a value from o to 20 from the drop-down box labeled Joint Roughness Coefficient. The JRC has no units.

Values of JRC range from o for smooth joints to 20 for rough joints with many asperities (Barton 1973). Citing Barton et al. (1985), Dr. Ebeling considers that 15 is a typical upper value for JRC.

Hydraulic conductivity of the rock joint: The user-provided value of the JRC, in conjunction with the user-provided value of mechanical aperture, $E$, are used to compute a value for conducting aperture, $e$, for the rock joint(s). An interrelationship developed by Barton et al. (1985), as discussed in section 2.3.3 of Murphy et al. (2002), is used to estimate the joint's conducting aperture, $e$. The Barton et al. (1985) relationship between, $e, E$, and JRC is valid for SI units (i.e., apertures in $\mu \mathrm{m}$ ) and valid only for values of $\mathrm{E} \geq \mathrm{e}$ and with a range of mechanical aperture from 1 to $1,000 \mu \mathrm{m}$. Recall the user establishes the units of the problem early on in CAS_Stab-R model development (Figure 3-8). CAS_Stab-R accommodates English units and converts mechanical aperture, $E$, from inches to SI units of $\mu \mathrm{m}$ before using the Barton et al. (1985) relationship to calculate the value for conducting aperture, $e$ (in units of $\mu \mathrm{m}$ ). With a value for the rock joint's conducting aperture, $e$, the cubic law is then used to define the hydraulic conductivity of 
the rock joint, using the relationship given in section 1.4.1 in Ebeling et al. (2012). The resulting values are then converted back to the user-specified coordinate system, either SI or English, to continue the CAS_Stab-R computations.

\subsubsection{Region - Material Mapping section}

This section of the Materials tab is the means by which material properties are assigned to the defined regions of the model. The section contains a list of drop-down selection boxes for the structure and all defined regions of the model. Figure 3-53 shows an example of a model for which three material regions have been defined. Using the drop-down selection boxes, the structure has been mapped to Material 4; Region 1 has been mapped to Material 3; Region 2 to Material 2; and Region 3 is currently being assigned Material 1.

Figure 3-53. Region - Material Mapping example.

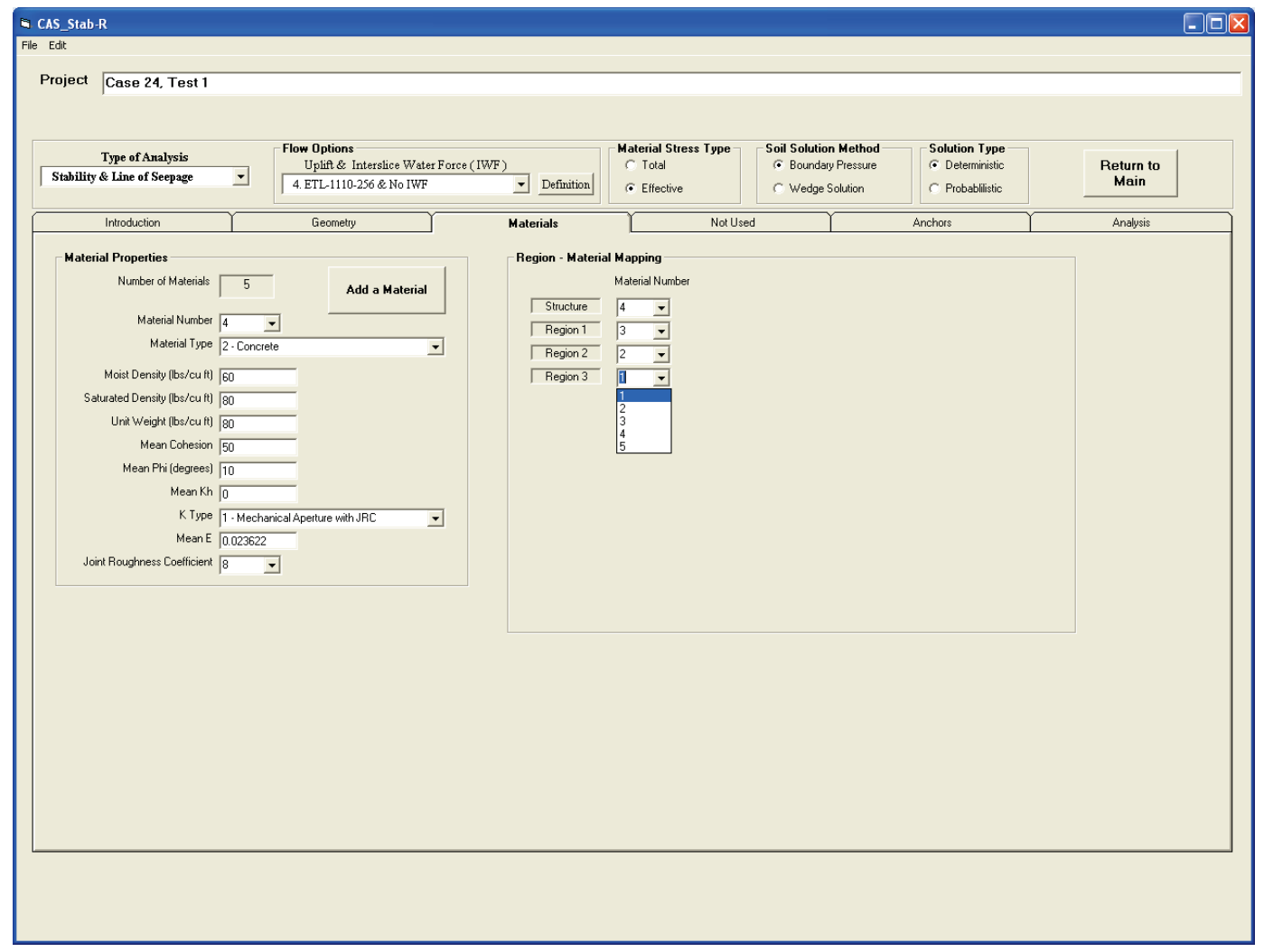




\subsubsection{Anchorage tab}

The Anchorage tab is shown in Figure 3-54. This tab provides the means to select and give values to the parameters that will affect the reduced cable capacity of the anchors during simulations.

Figure 3-54. Anchorage tab.

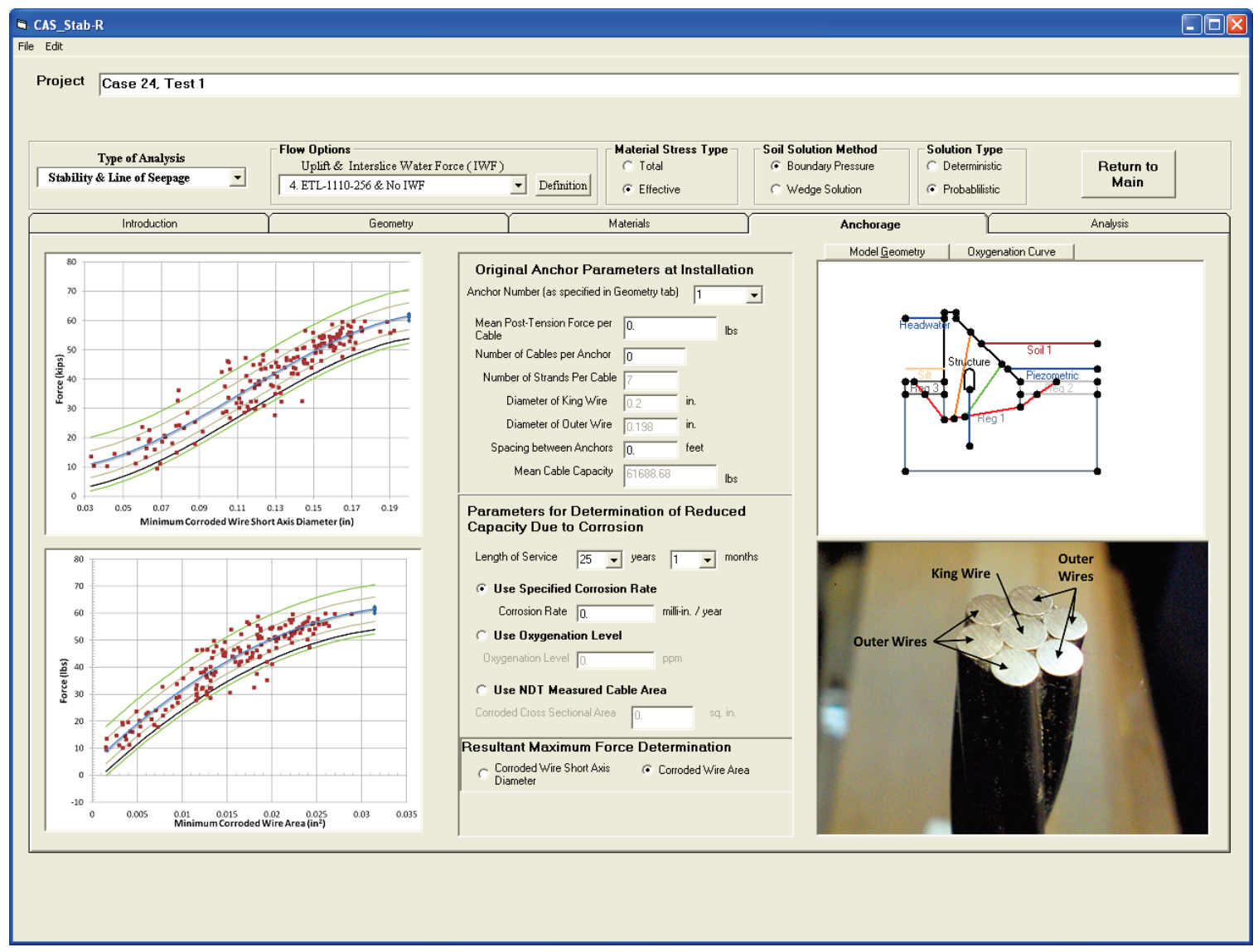

The left third of this tab contains two plots relating the corroded cable size to the rupture force during cable pull-test experiments conducted at ERDC. The top plot relates the short axis diameter of the cable rupture location to the rupture force. The bottom plot relates the minimum corroded area at the rupture location to the rupture force. Detailed information on the pull tests is given in Ebeling et al. (2016).

The middle third of the tab going from left to right provides the input locations for the parameters that will affect the reduced capacity of the cable. This section is further broken into three input areas. The top input area contains the inputs for the pristine cable information. This area is labeled Original Anchor Parameters at Installation. There is a drop-down 
box used to select the anchor for which parameters are to be specified. The anchor numbers correspond to those in the Geometry Anchors tab. The mean value of the post-tension force applied to the cable upon installation is entered into the box labeled Mean Post-Tension Force per Cable. Only the mean value that can be used deterministically is entered here. Probabilistic values to be used in generating the simulation vectors are entered using the Edit - Probability Distributions menu option. The number of multi-strand cables that are grouped into an anchor is entered in the box labeled Number of Cables per Anchor. The distance between each anchor is entered in the box labeled Spacing Between Anchors.

The pull tests were performed on seven-strand cables with a mean diameter of the king wire of $0.200 \mathrm{in}$. and mean diameter of outer wires of $0.198 \mathrm{in}$. The pull tests performed on the pristine-condition cables produced a mean rupture force of $61,688.68 \mathrm{lb}$. As the data available for reduced capacity apply at this time to only this type of cable, the input boxes for these values are disabled and reserved for future use.

The area labeled Parameters for Determination of Reduced Capacity Due to Corrosion is used to specify the method and parameter values for determining the reduced capacity of the anchors. Use the drop-down boxes labeled Length of Service to select the length of time in years and months the structure has been in service. The user has a choice of three methods to compute the remaining cable area:

1. Use Specified Corrosion Rate

2. Use Oxygenation Level

3. Use NDT Measured Cable Area.

The desired method is selected by clicking the appropriate option button. Then enter the mean value for the corrosion rate, oxygenation level, or NDT measured area in the appropriate input box. Only the mean value that can be used deterministically is entered here. The probabilistic value to be used in generating the simulation vectors is entered using the Edit Probability Distributions menu option.

The user must select an option from the area labeled Resultant Maximum Force Determination. This option selects the curve fit to use in computing the maximum capacity remaining in the cable, either the Short Axis Diameter curve (seen in the upper left of the tab) or the Corroded Wire 
Area (seen in the lower left of the tab). Select the desired option by clicking the appropriate option button.

The right third of the tab is separated into two areas. The top area is a display box with two buttons at top left. Clicking the Oxygenation Curve button will display a curve that shows the relationship of oxygenation level in parts per million to the corrosion rate in microns/year. This curve display is provided for the user's information. The display with the Oxygenation Curve selected is shown in Figure 3-55.

Clicking the Model Geometry tab will provide a drawing of the model with the selected anchor highlighted in the color orange as shown in Figure 3-54. This provides the user with a visual to ensure the desired anchor has been selected.

Figure 3-55. Anchorage tab with Oxygenation Curve displayed.

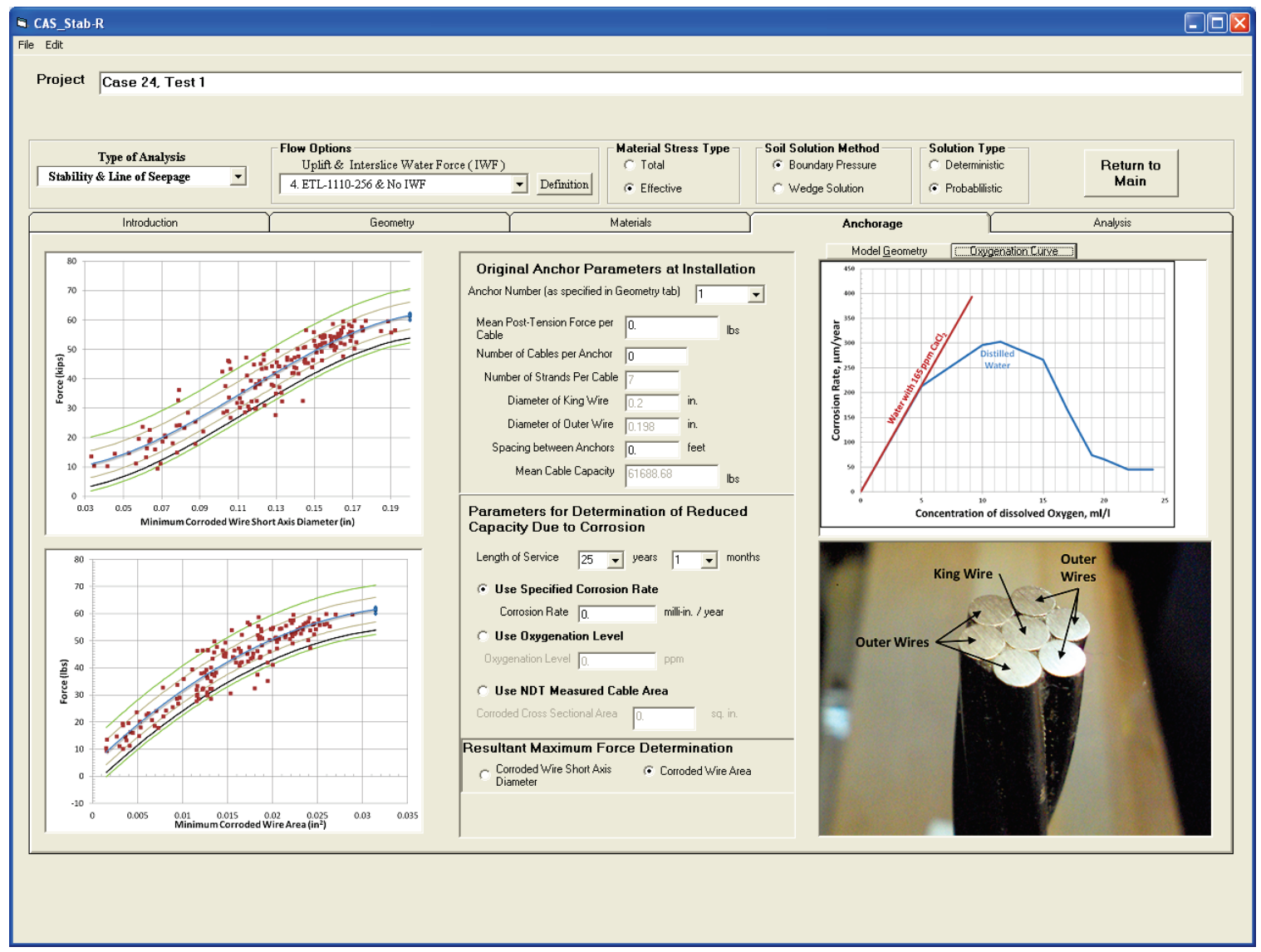


The bottom area is a graphic of the cut end of a pristine section of cable with the king wire strand and outer wire strands labeled. This is provided to avoid any confusion as to which strand is called the king wire and which strands are the outer wires.

\subsubsection{Analysis tab}

Clicking the Analysis tab will produce the screen shown in Figure 3-56 or Figure 3-57 depending on the selection of a Probabilistic or Deterministic solution in the Solution Type box located just above the Anchorage tab. The two types of analysis are discussed in the following sections. The deterministic solution is discussed first as it is the simplest solution.

Figure 3-56. Initial Analysis tab - probabilistic solution.

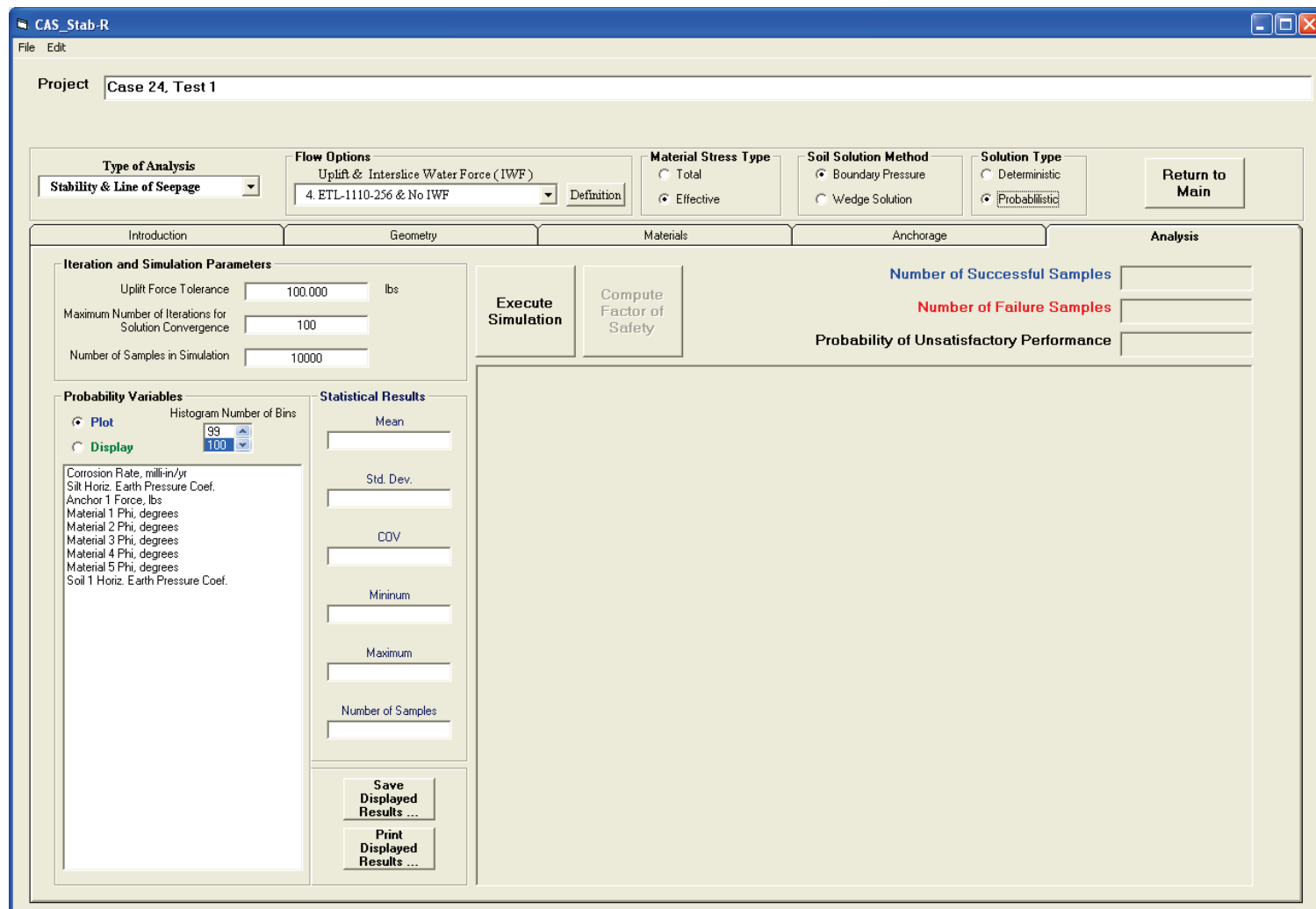


Figure 3-57. Initial Analysis tab - deterministic solution.

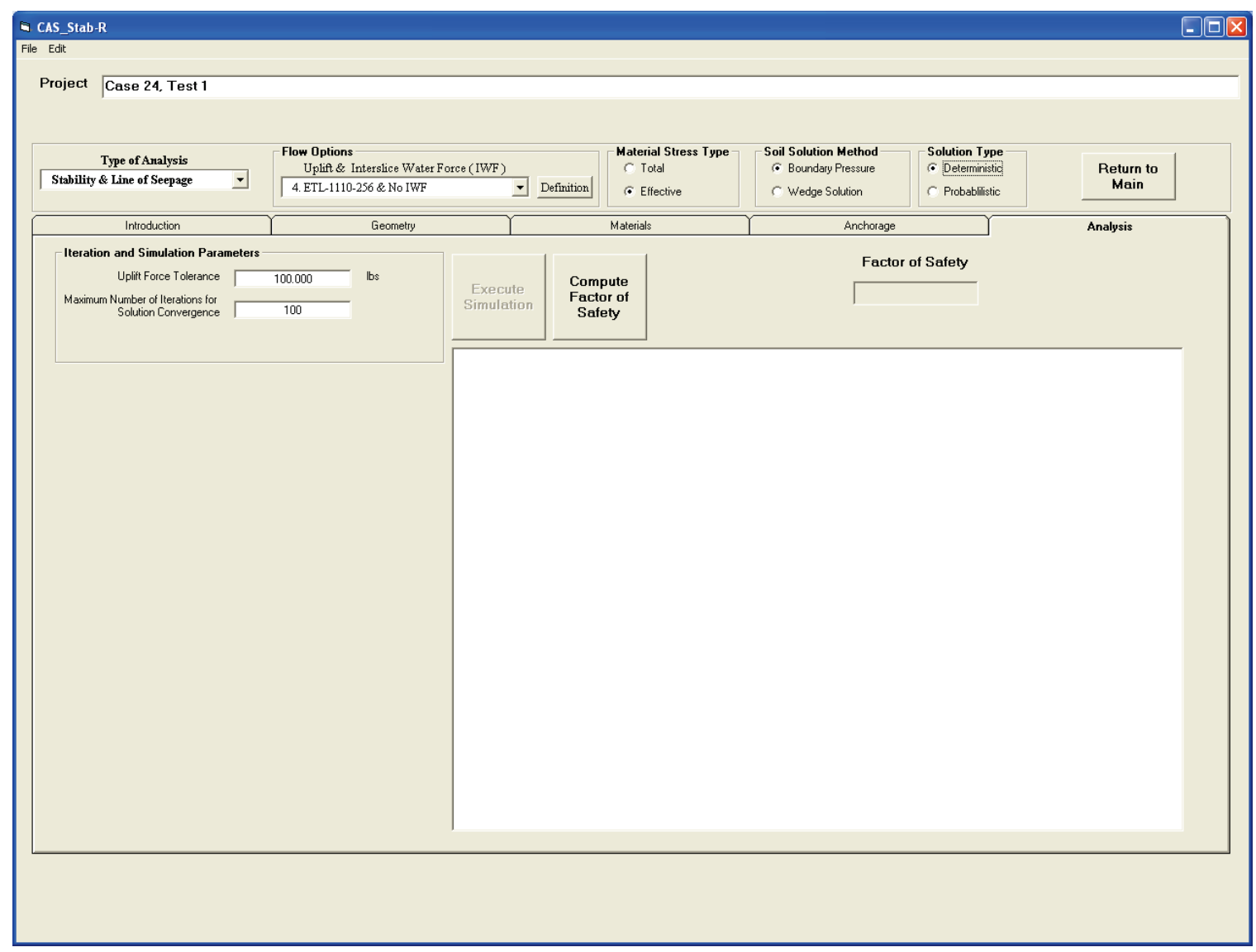

\subsubsection{Deterministic solution}

If a deterministic solution has been chosen, the analysis performs a single computation of FOS utilizing the mean values of the probabilistic variables (lock-off anchor force, chosen corrosion value, material cohesion, material internal friction angle, phi, material earth pressure coefficient, soil earth pressure coefficient, silt earth pressure coefficient).

Two parameter values for the FOS computation must be entered on this tab. They are in the Iteration and Simulation Parameters box in the upper left corner of the tab. One parameter is the uplift force tolerance. This is the value the computed uplift force must be less than to consider equilibrium achieved. The second parameter is the maximum number of iterations for solution convergence. If equilibrium has not resulted within the entered value for this parameter, the model is either totally unstable (FOS much less than 1.0) or excessively conservative (FOS much greater than 1.0). 
To determine the FOS for the current model and parameters, click the button labeled Compute Factor of Safety. The computed FOS will display in the box titled Factor of Safety. The FOS is computed using the wedge solution method detailed in ETL 1110-2-256 (HQUSACE 1981).

\subsubsection{Probabilistic solution}

When executing a probabilistic solution, a set of randomly generated vectors for the probabilistic variables is generated. The FOS is computed for the model for each vector, and the FOS are saved and used to compute the PUP.

When a probabilistic solution has been chosen, the tab contains the input and output boxes observed in Figure 3-56. The various inputs and outputs are discussed in the following sections.

\section{Iteration and simulation parameters}

The parameters in this frame are those necessary for the FOS computation and the generation of simulation vectors. The parameters for the FOS computation are the uplift force tolerance and the maximum number of iterations for solution convergence. Those two parameters are discussed in the report section 3.4.8.1 above. The third parameter in this frame is the number of samples to be generated for the simulation. Enter a value for this parameter in the input box labeled Number of Samples for Simulation.

\section{Execute simulation button and simulation results}

After the model has been defined and the desired simulation parameters have been entered, a simulation can be performed by clicking the Execute Simulation button. After clicking this button, the Reliability Running popup window shown in Figure 3-58 will appear as an indication that an ASM computation is in progress to determine the point about which the simulation variables will be centered. The ASM computation finds the vector that originates at the point defined by the user-supplied mean variable values and extends to the nearest point of the limit state function. The limit state function $G(z)$ is the FOS for the ETL 1110-2-256 (HQUSACE 1981 ) wedge solution equal to 1 . The point on the $G(z)$ function is the point at which the Dakota software will center the simulation variable values. The ASM algorithm is detailed in Ebeling et al. (2013). 
Figure 3-58. Determination of ASM Design Point indication.

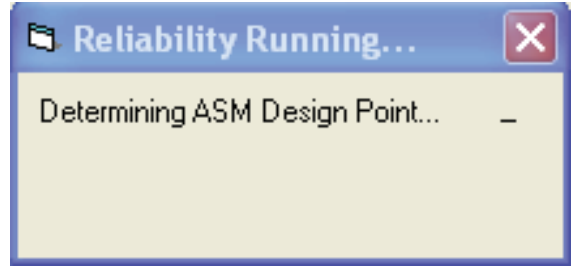

Following the determination of the ASM design point, Dakota software generates the simulation variable values. The Reliability Running window will inform the user that the simulation is in progress. This window will now include a progress bar indicator as shown in Figure 3-59.

Figure 3-59. Running Simulation indication.

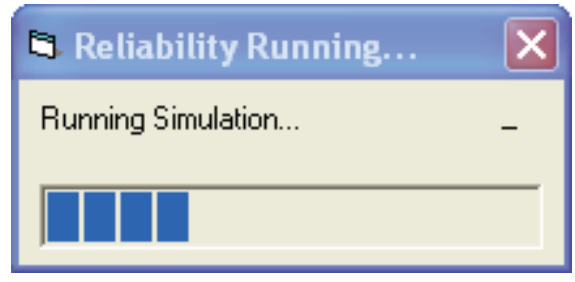

Execution time varies based on the number of samples selected. Upon completion, the results of the simulation are shown in the boxes in the upper right corner of the tab as shown in Figure 3-60.

The Number of Successful Samples (shown in blue) is the number of samples that resulted in an FOS of greater than 1.o. The Number of Failure Samples (shown in red) is the number of samples that resulted in an FOS less than or equal to 1.o. The PUP (shown in black) is the Number of Failure Samples divided by the total Number of Samples. 
Figure 3-60. Sample simulation results.

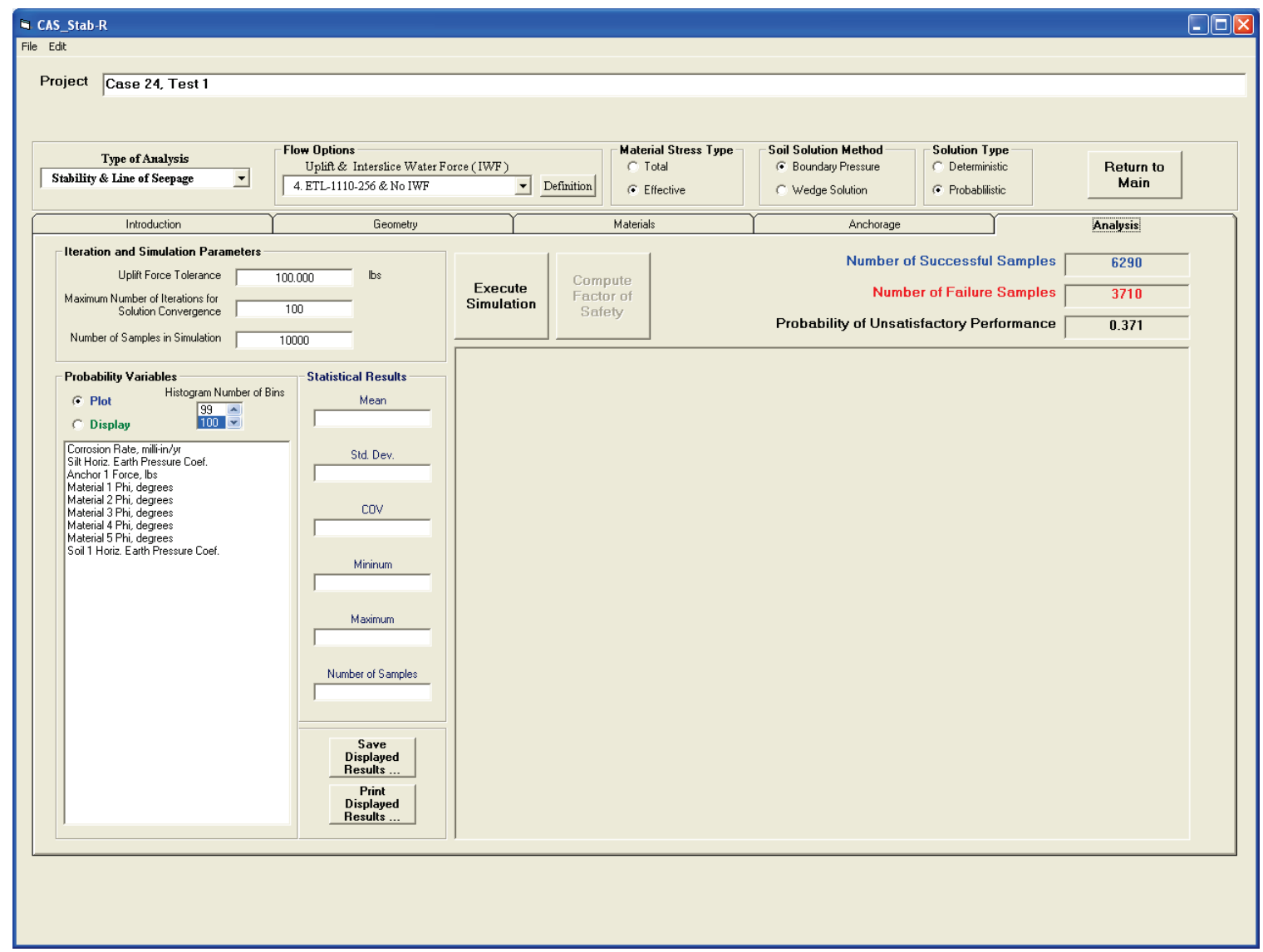

\section{Probability variables}

This frame contains a box with (1) a list of the probability variables that were inputs for the simulation, (2) option buttons to select viewing of histograms or a text listing of the histogram data and individual samples of the probability variables, and (3) a list box to select the number of bins when plotting histograms. Histograms and data values appear in the display box on the right side of the tab. To view a histogram of a variable's values, click the option button labeled Plot. Then, click on the variable in the list box in the bottom of the frame. In example, clicking on the variable labeled Anchor 1 Force, lbs produces the display shown in Figure 3-61.

Clicking the Display option button produces a list box that contains the histogram data values and the individual sample values of the selected probability variable. An example of the Display option is shown in Figure 3-62. Use the scroll bar on the right of the Display box to view all the values. 
Figure 3-61. Histogram display of an anchor lock-off load input variable.

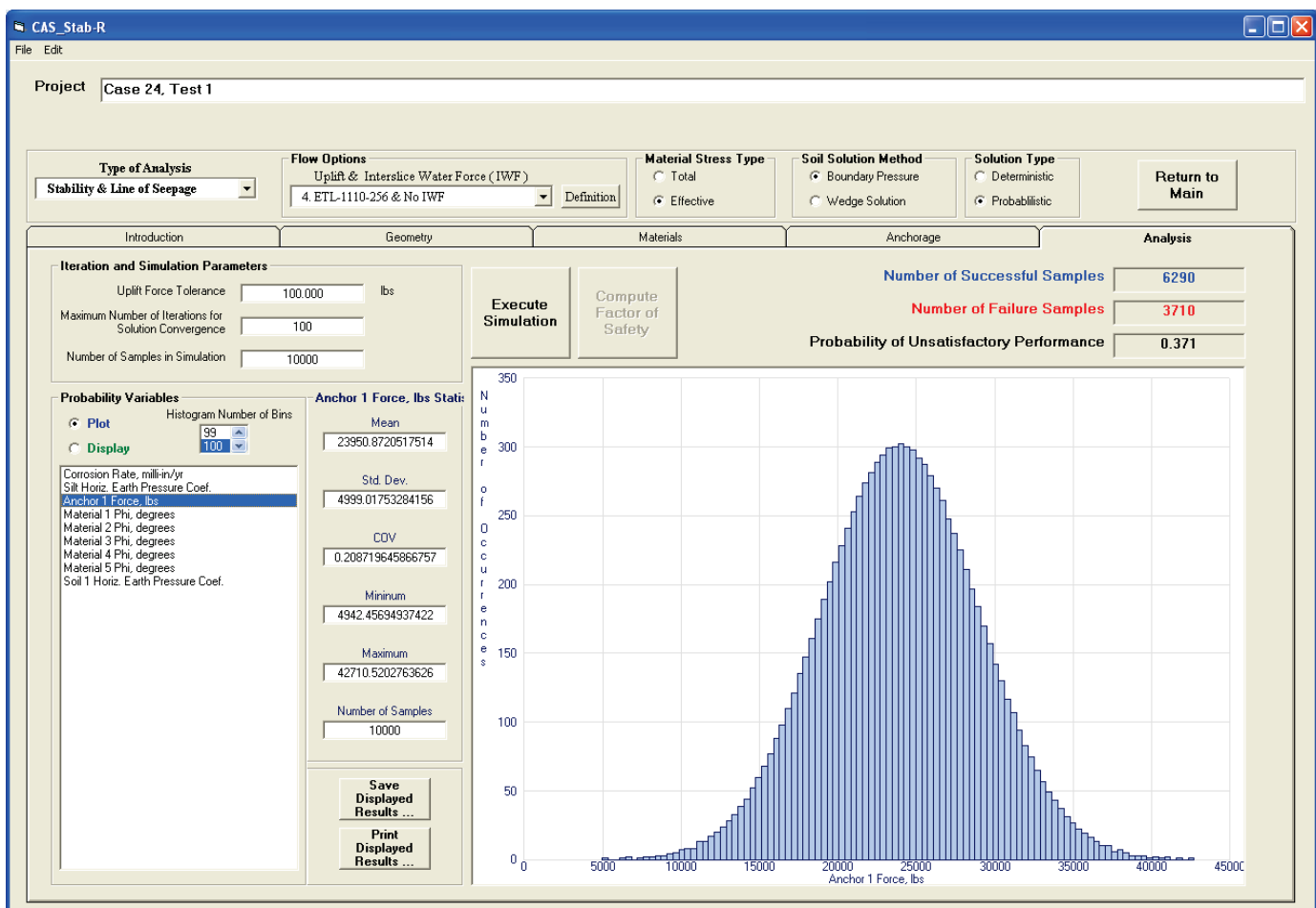

Figure 3-62. Text display of an anchor lock-off load probability variable.

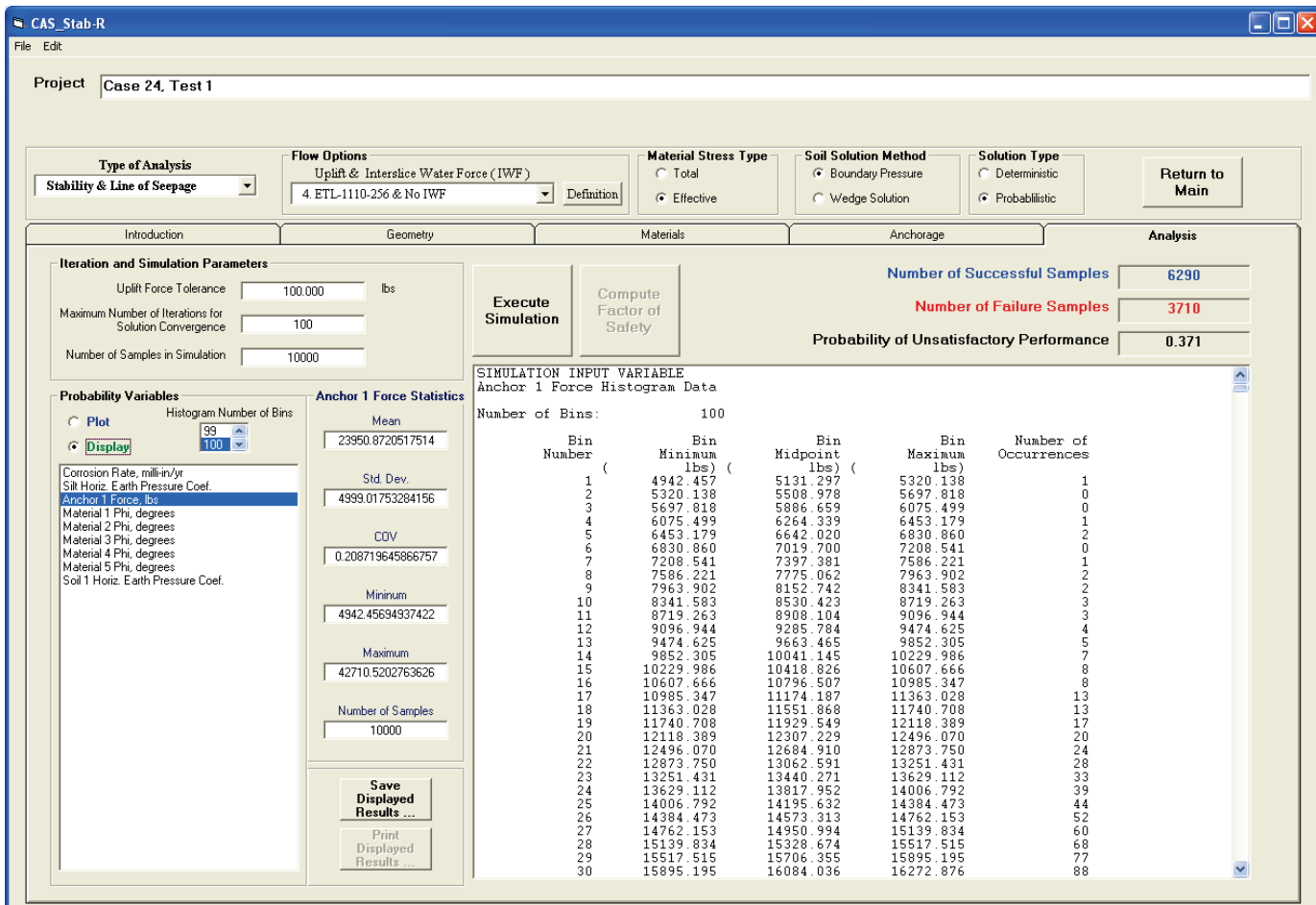




\section{Statistics box}

The statistics for the currently displayed text values or histogram plot are displayed in the box to the right of the Probability Variables. As seen if Figure 3-62 above, the statistics displayed are the mean, standard deviation, $\mathrm{COV}$, minimum, maximum, and number of samples.

\section{Save options for probability variable data}

Two buttons are used to save histogram plots or text data sets of the probability variables. They are located below the Statistics box and can be seen in Figure 3-62. The top button is labeled Save Displayed Results. Clicking this button produces a File Save dialog window as shown in Figure 3-63. Select the location and the desired file name. Then, click on the Save button. The file will contain the information that is shown in the Display box when the Probability Variables box Display option button has been selected such as that seen in Figure 3-62 above.

Figure 3-63. Probability Variables Save As dialog box.

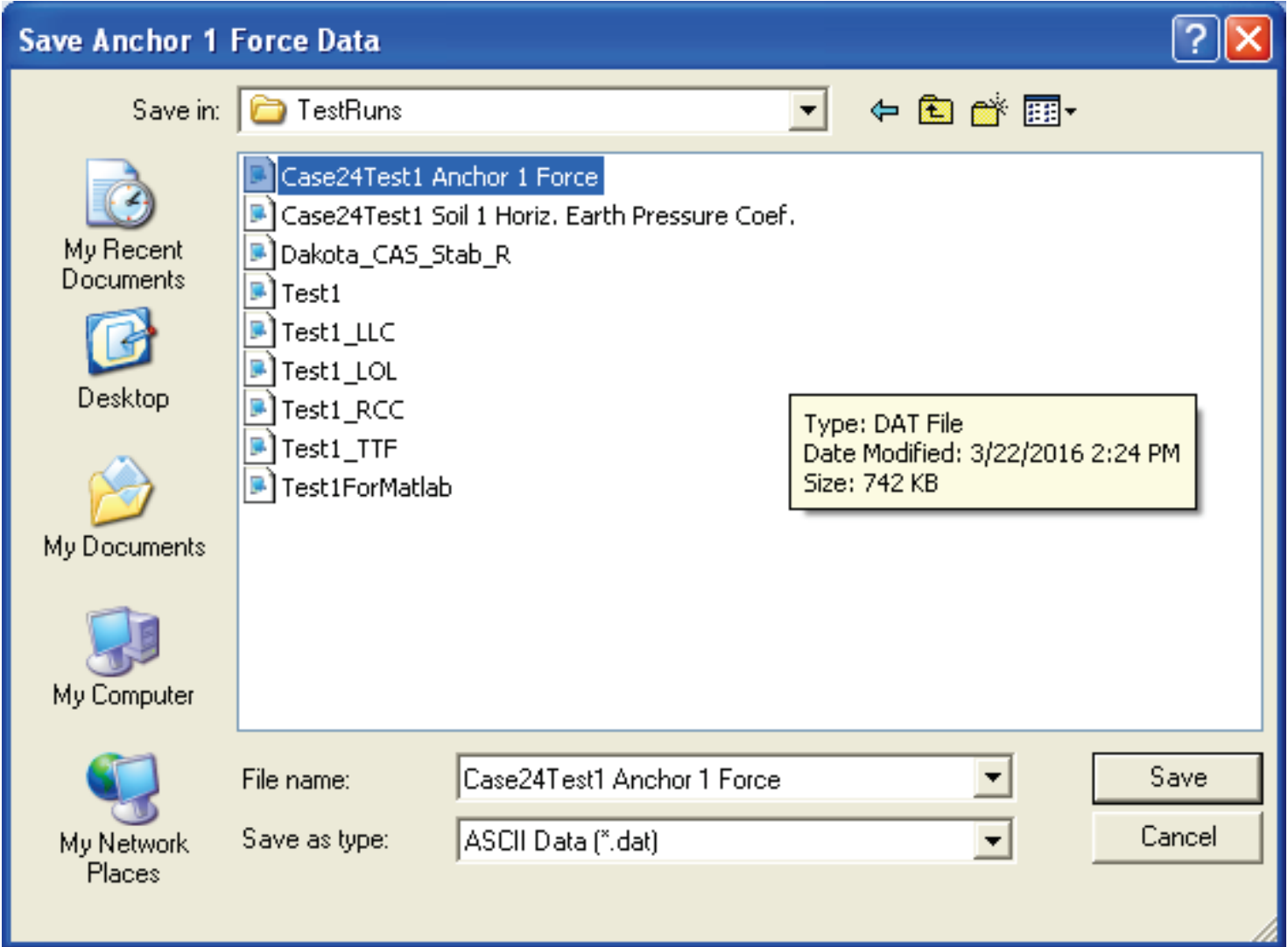


The bottom button is labeled Print Displayed Results. This button is only enabled when a histogram plot is shown in the Display box. Clicking this button will produce a Print dialog box similar to the one shown in Figure 3-64. Select the appropriate printer device and click the Print button.

Figure 3-64. Print dialog box for printing a Probability Variables histogram.

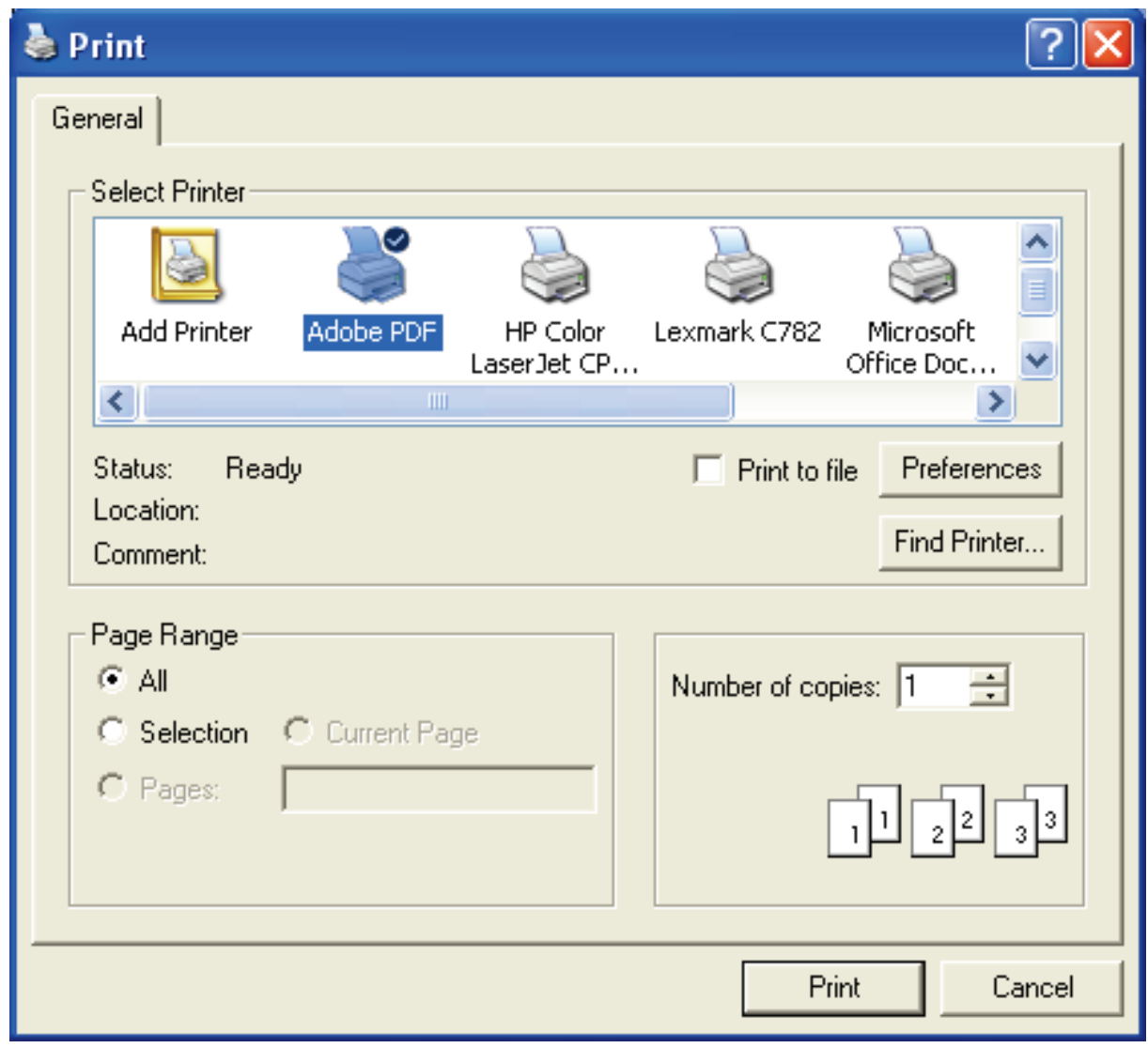

\subsubsection{Analysis options}

CAS_Stab-R provides options in how seepage, material stresses, and soils are modeled. The user also selects the type of analysis to be performed, deterministic or probabilistic.

The means for setting these options are located below the Project input box at the top of the window. The option selection controls are shown in Figure 3-65.

A drop-down list box that is labeled Type of Analysis is seen in Figure 3-65. At this time, this list box contains only one option, Stability and Line of Seepage. Presently, CAS_Stab-R only performs this type of 
analysis. The list box is included in the program to provide for future implementations of analysis type.

\subsubsection{Flow Options}

The Flow Options box contains a drop-down list of methods implemented in CAS_Stab-R to compute the uplift wedge forces and interslice water pressures. The three methods implemented are shown in Figure 3-65. Although three methods are available, they are labeled 4, 5, and 6. The three methods are a subset of flow options supported in an earlier ERDC software GDLAD_Foundation (Ebeling et al. 2012) and maintain the same numbering convention for consistency.

Figure 3-65. Flow Options drop-down list.

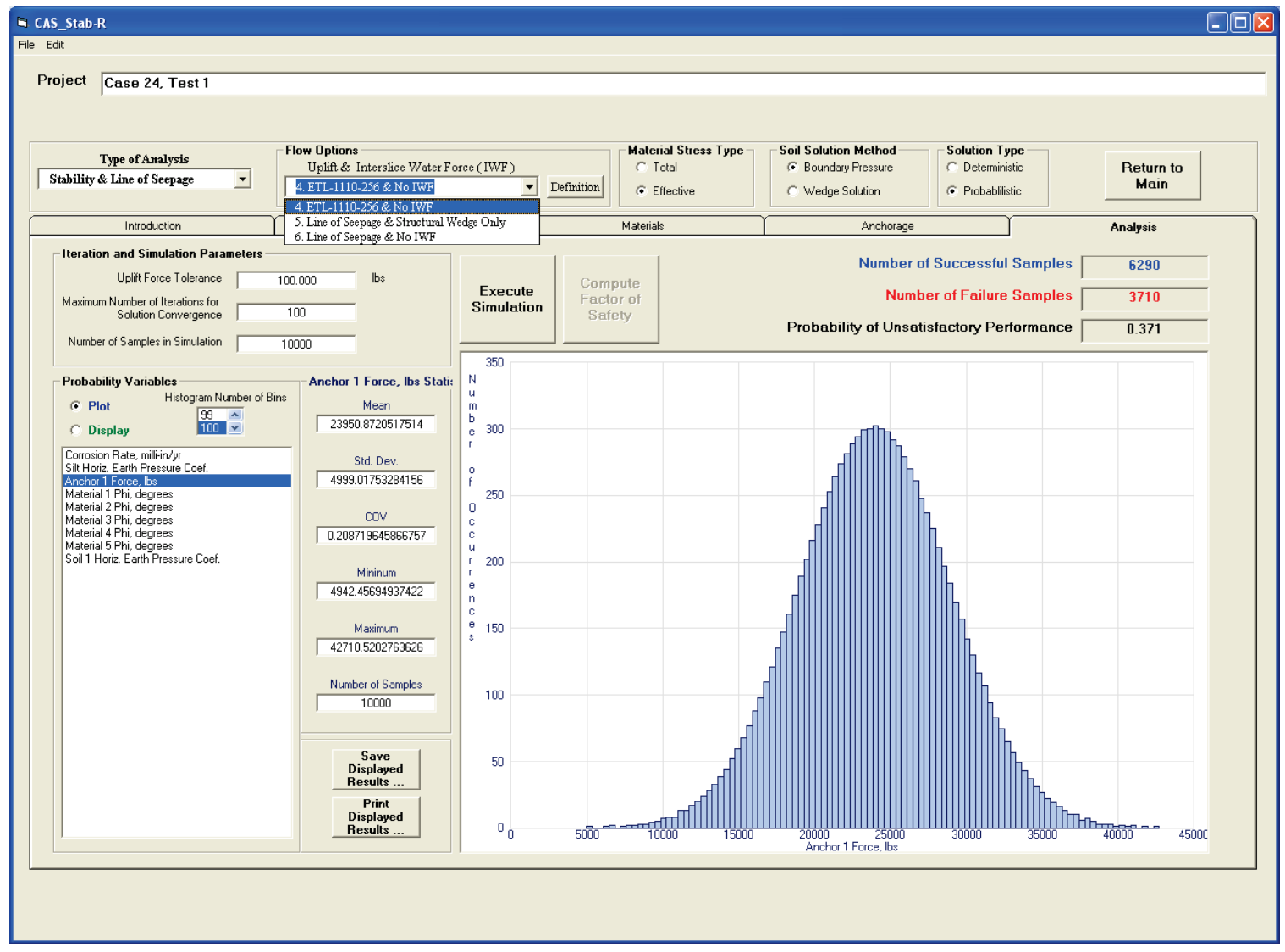

Flow Option 4 computes uplift wedge forces as presented in the ETL-11102-256 (HQUSACE 1981) with no interslice water forces. The seepage regime that defines the total heads along the perimeter of the structural wedge embedded within the foundation is assumed to occur solely along the rock-to-concrete foundation interface that forms the perimeter of the structural wedge using this flow option. As stated in EM 1110-2-256, "the 
depth of cracking in massive strong rock foundations should be assumed to extend to the base of the structural wedge ... and full hydrostatic pressure should be assumed at the bottom of the crack." In this procedure, all head loss occurs along the base of the structural wedge. Hydrostatic water pressures are assumed along the driving and resisting wedges potential slip planes. This assumption means that the total heads along the base of the resisting wedge(s) are equal to the total head of the water surface above them. Figures 6.14 and 6.15 in Ebeling et al. (2012) depict this Flow Option 4 configuration in terms of total head. The total heads along the potential slip plane of the structural, driving, and resisting wedges are converted into pressure heads using the Bernoulli equation (Equation 1.5 in Ebeling et al. [2012]), assuming a negligible velocity head. The pore water pressures acting normal to the potential slip plane are determined from the resulting pressure head distribution using Equation 1.6 in Ebeling et al. (2012). There is no discontinuity in values of total head, pressure head, or pore water pressure at the slip plane boundaries where the driving wedge meets the structural wedge (i.e., structural wedge heel) and where the structural wedge meets the resisting wedge (i.e., structural wedge toe).

Flow Option 5 blends two hydraulic subsystem models; one model affects the structural wedge, and the other affects the driving and resisting wedges. First, a steady state, line of seepage condition (Appendix $\mathrm{C}$ in Ebeling et al. [2016]) is assumed along the perimeter of the structural wedge where the structural wedge is in contact with the rock foundation. This line of seepage analysis defines the total head along this perimeter. In this procedure, head loss occurs along the side of the structural wedge from the ground surface to the base of the structure along the high-head side, along the base of the structural wedge, and up along the side of the structural wedge from the structural base to the surface on the low-head side. The head conditions along the base of the structure only are extracted from this perimeter seepage analysis establishing the total head, pressure head, and water pressures for the base of the structural wedge. For the driving and resisting wedge potential slip planes, hydrostatic water pressures are assumed, as in Flow Option 4. Recall, this assumption means that the total head for a point on the slip planes of the driving and resisting wedge(s) is equal to the total head of the water surface above that point. With the blended model, there is likely to be a discontinuity in values of total head, pressure head, or pore water pressure at the slip plane boundaries where the driving wedge meets the structural wedge (i.e., 
structural wedge heel) and where the structural wedge meets the resisting wedge (i.e., structural wedge toe). Figures 6.17 and 6.18 in Ebeling et al. (2012) depict this Flow Option 5 configuration in terms of total head. Interslice water forces are extracted from the line of seepage analysis at the two faces of the structural wedge but not within the other driving and structural wedges, as depicted in Figure 6.9 of Ebeling et al. (2012).

Flow Option 6 computes seepage only along the wedge bases using the line of seepage with no interslice water forces. The potential slip plane corresponds to a continuous series of connected rock joints from upstream to downstream of the gravity dam. Fluid flow occurs only along this singular joint path system. All of the joints are assumed to have the same value for mechanical aperture and JRC. Consequently, they all have the same value for hydraulic conductivity. A (steady state) line of seepage methodology is used to compute the distribution of total head along the entire length of potential slip plane defining the base of the driving, structural, and resisting wedges. Head loss occurs along the entire potential slip plane because of this steady state water flow within the rock joint(s). This establishes the total head, pressure head, and water pressures along the structural wedge base portion of the potential slip surface. Figures 6.20 and 6.21 in Ebeling et al. (2012) depict this Flow Option 6 configuration in terms of total head. There is no discontinuity in values of total head, pressure head, or pore water pressure at the slip plane boundaries where the driving wedge meets the structural wedge (i.e., structural wedge heel) and where the structural wedge meets the resisting wedge (i.e., structural wedge toe).

A table detailing the seepages for the currently selected flow option can be viewed by clicking the Definition button to the right of the Flow Options drop-down list. Remove the table from view by again clicking the Definition button. The table for Flow Option 4 is shown in Figure 3-66. 
Figure 3-66. Seepage table for Flow Option 4.

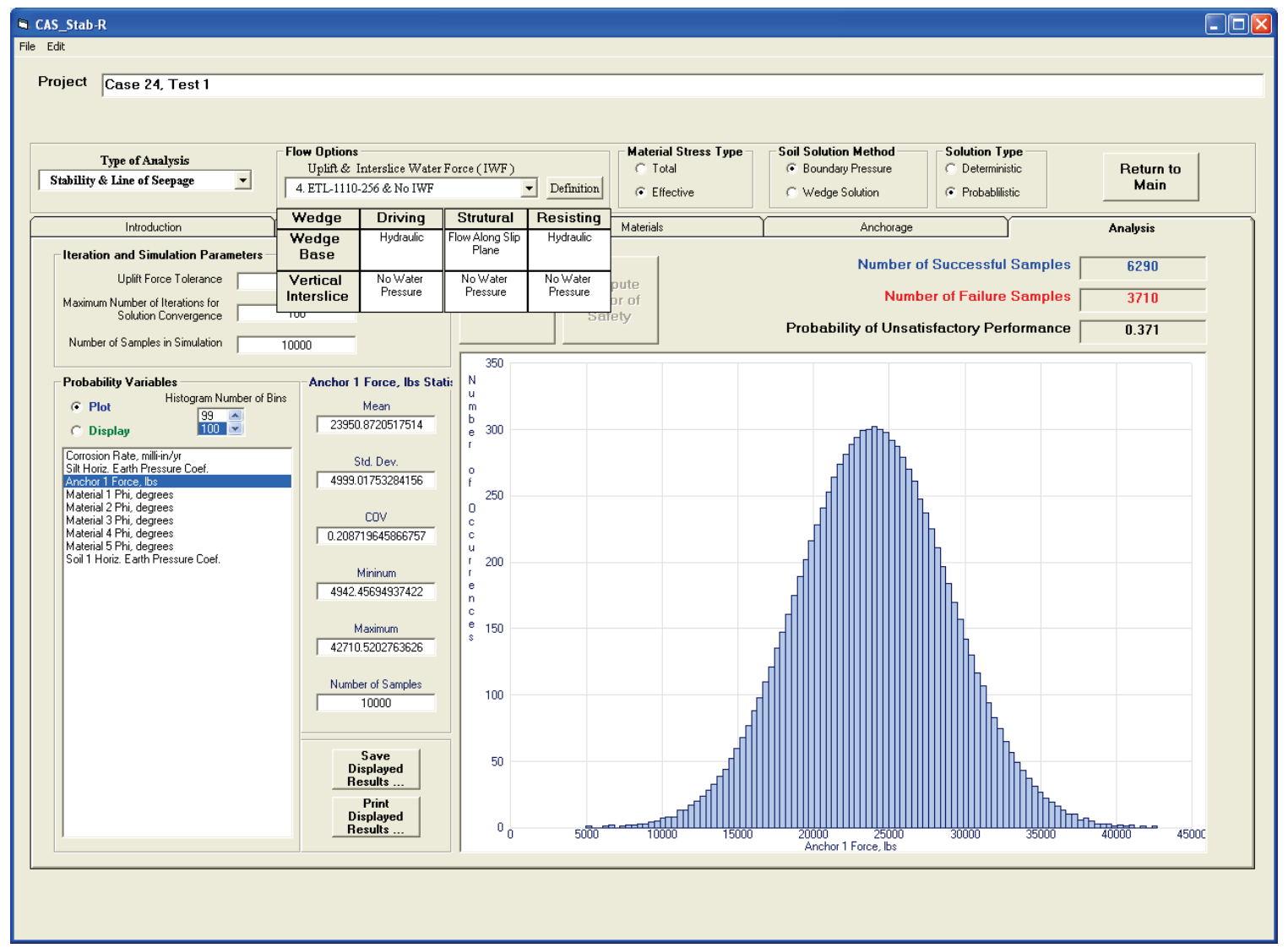

\subsubsection{Material Stress Type}

The Material Stress Type box allows the user to select either Total stress or Effective stress computations as the means of computing vertical forces in the material regions. This box is located in the top center of the window and is shown highlighted in Figure 3-67. Select the desired computation method by clicking the appropriate option button. 
Figure 3-67. Material Stress Type selection box location.

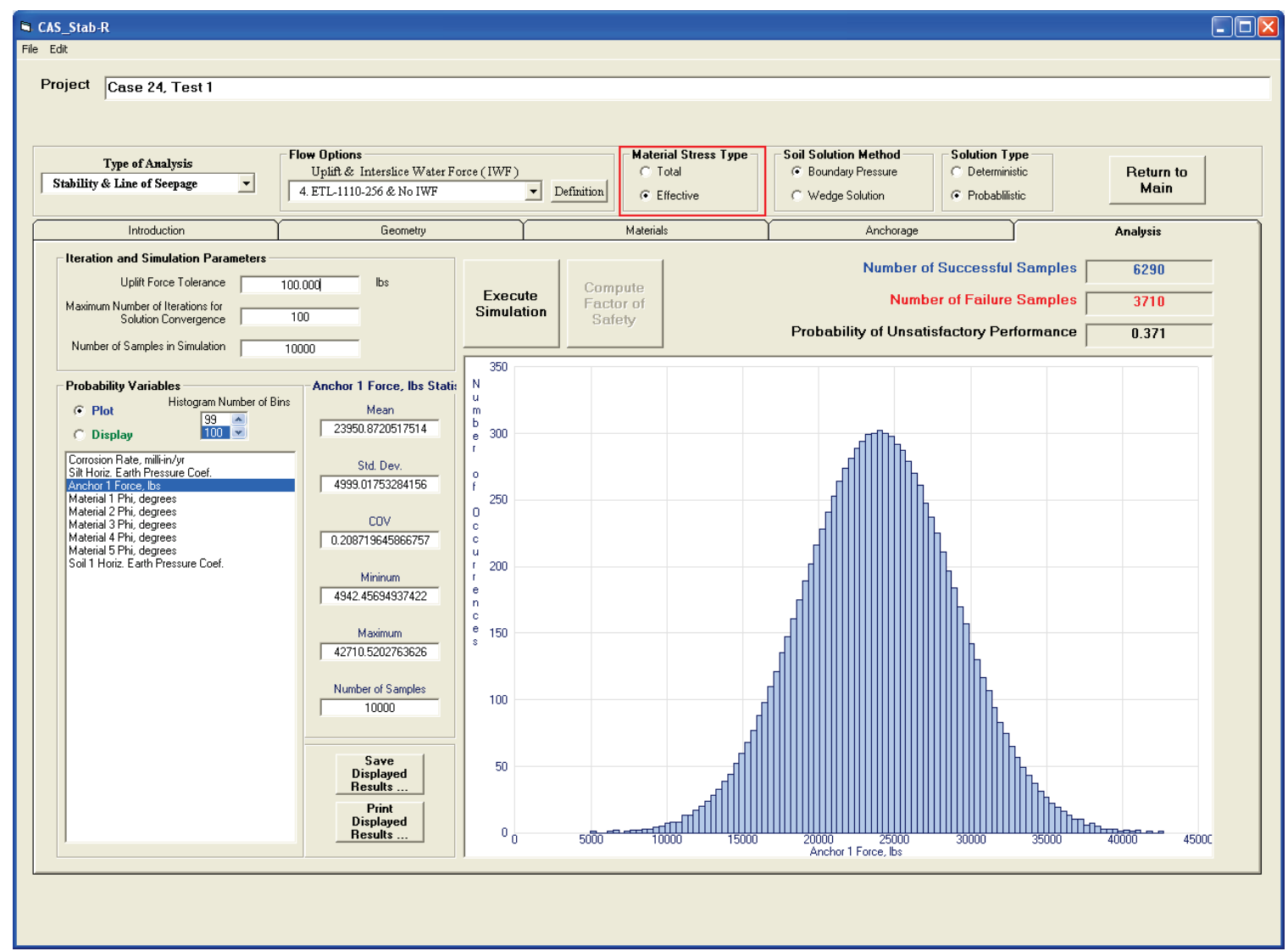

\subsubsection{Soil Solution Method}

Two methods are available in CAS_Stab-R for computing the effect of soils and silt on the model. The first method is to compute the boundary pressure applied to the structure by these materials. The second method is to include the silt and soils as materials in the same manner as rock region materials and include them as subwedges in wedge generation. The method to be used in the CAS_Stab-R computations is selected by choosing the Boundary Pressure option or the Wedge Solution option in the Soil Solution Method box shown highlighted in Figure 3-68. 
Figure 3-68. Soil Solution Method box location.

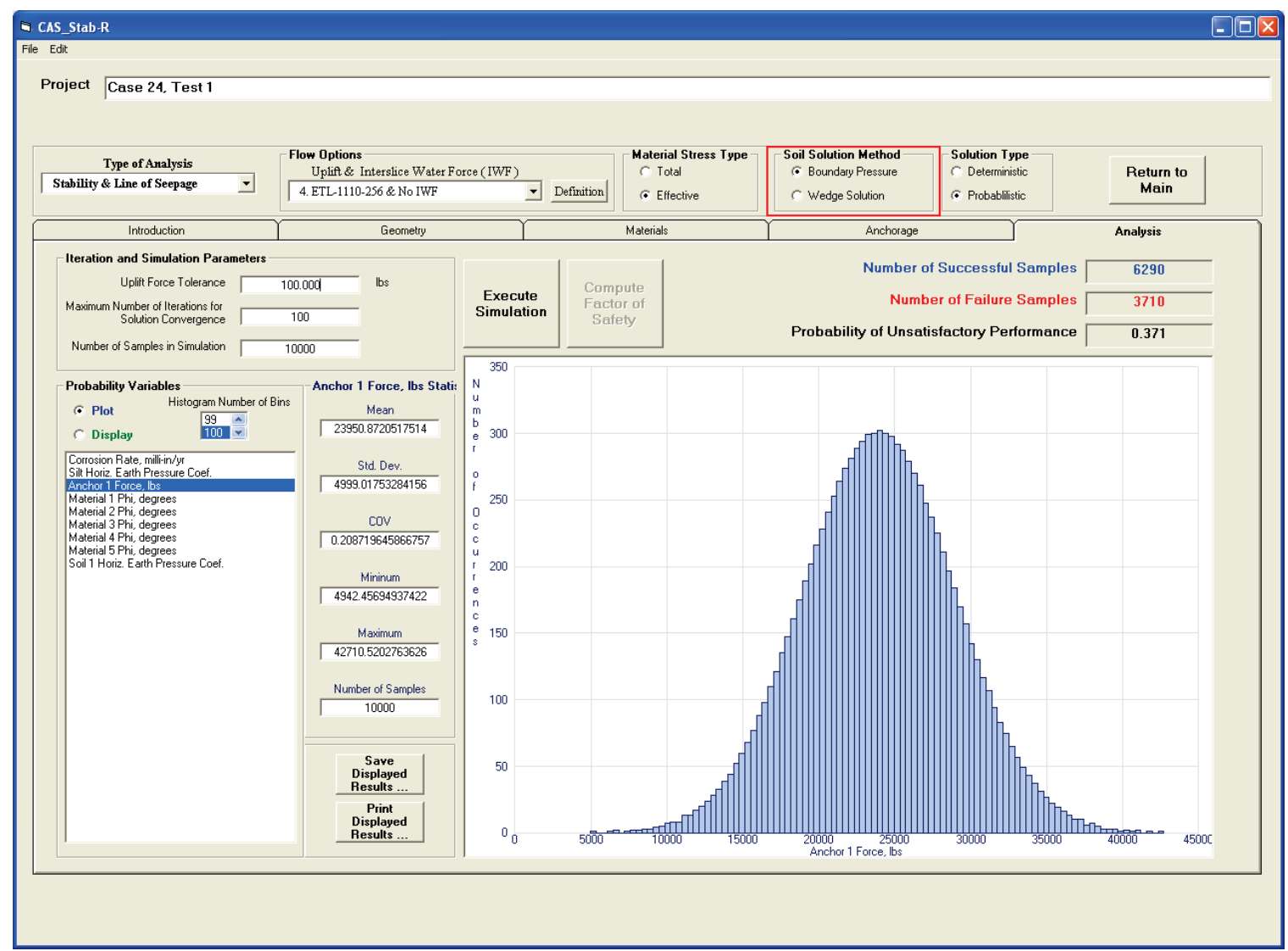

\subsubsection{Solution Type}

CAS_Stab-R allows the user to perform a probabilistic PUP simulation or to perform an FOS computation using the mean values specified for the probabilistic variables. The computation methodology is selected by choosing an option available in the Solution Type box highlighted in Figure 3-69. To perform the PUP simulation, select the Probabilistic option. To perform an FOS computation, select the Deterministic option.

\subsubsection{Return to Main button}

The Return to Main button in the upper right portion of the window shown in Figure 3-69 above is used to return to the CAS_Stab- $R$ main screen. The main screen is shown in Figure 3-1. From there the user can choose from the two main functions of CAS_Stab-R: a probabilistic remaining anchor life determination or a structural stability determination. 
Figure 3-69. Solution Type box location.

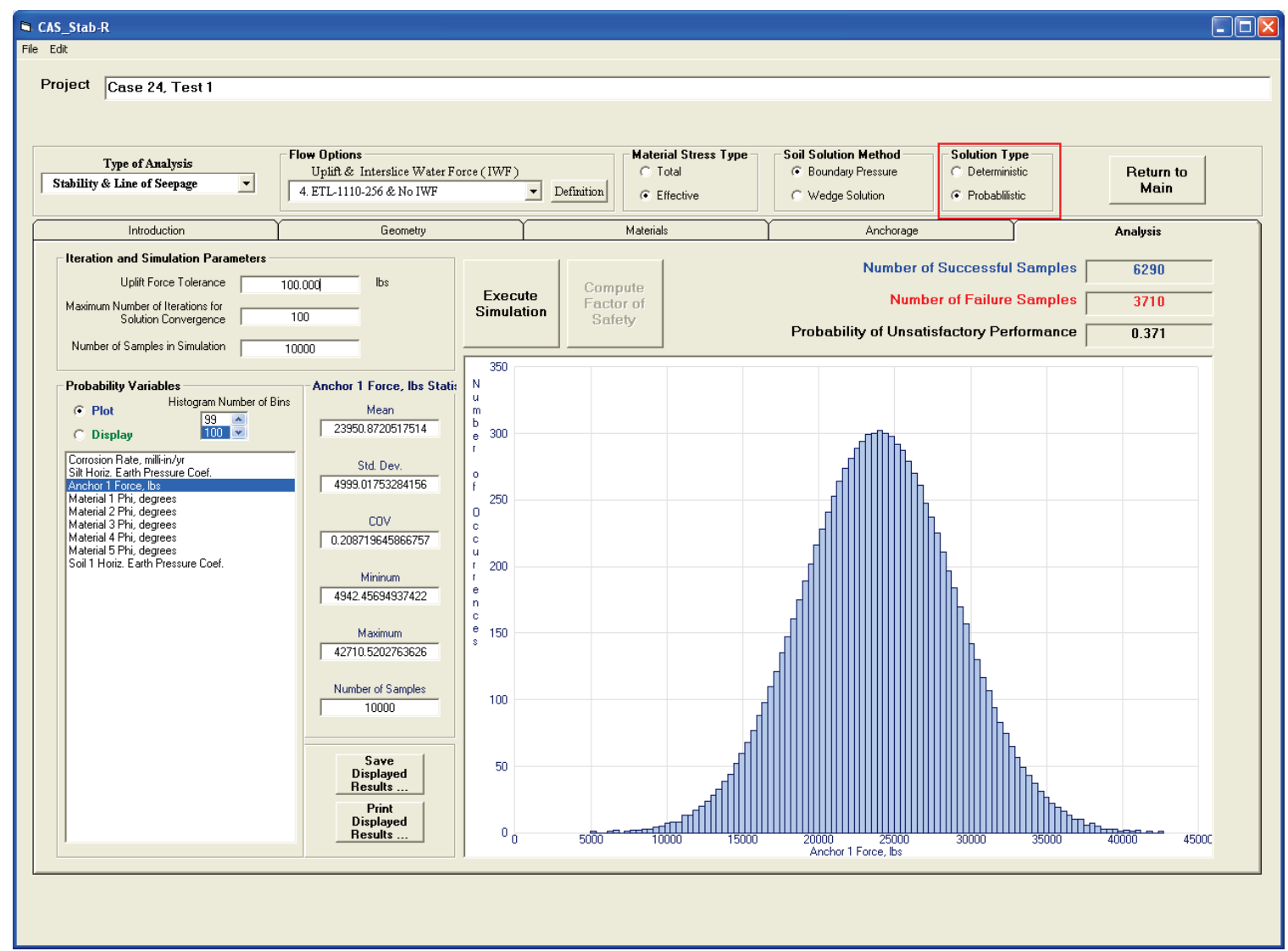

\subsection{Lifetime of Anchor (LoA)}

The LoA option provides a means of entering information for a multistrand anchor cable and corrosion parameters and then analyzing the input to generate a probabilistic estimate of RAL and POF after a userselected LOS. The input needed to perform an analysis of this type is the following:

- the initial tensile force applied (lock-off load)

- corrosion specification method and parameters

- length of time the anchor has been in service.

Clicking this option button on the window shown in Figure 3-1 loads the tabbed input window labeled Probabilistic Estimate of Remaining Anchor Life shown in Figure 3-70. This window will be referred to as the RAL window. The various components of this window are discussed in the following subsections. 
Figure 3-70. Initial RAL window.

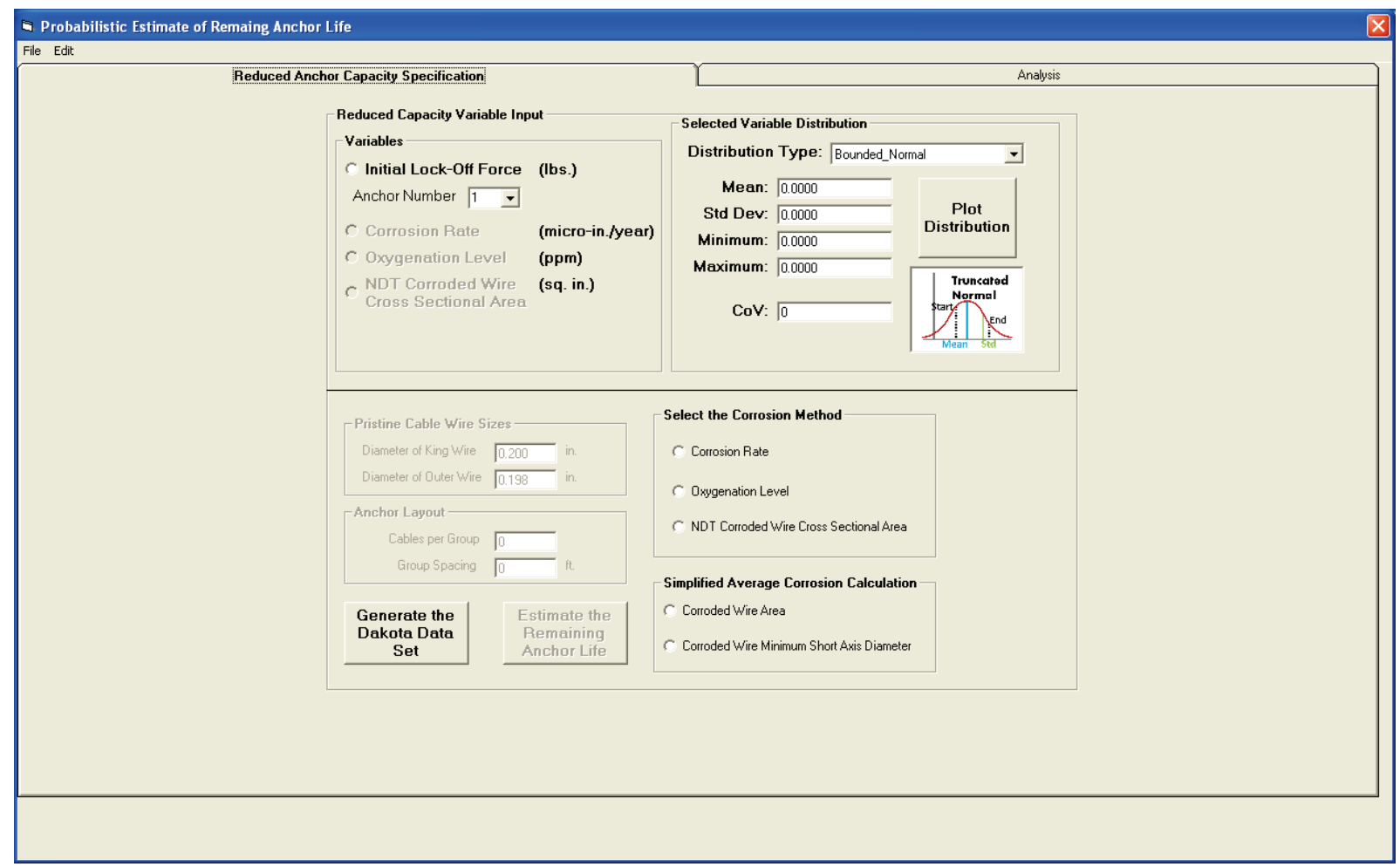

\subsubsection{File menu}

The RAL window has File menu options as shown in Figure 3-71. The File menu provides options to load an RAL configuration from an existing file (Open), to save the current cable configuration to a file (Save and Save As), and to exit the RAL window (Exit).

Figure 3-71. RAL

window, File menu.

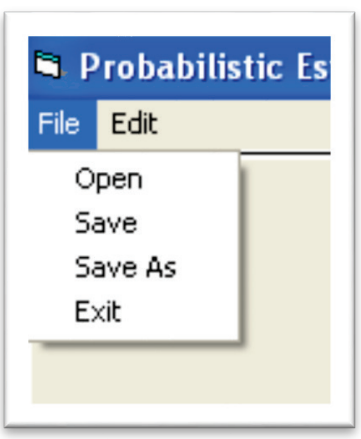




\subsubsection{File - Open}

Choose the File - Open option to load a previously saved RAL file. Clicking the Open option will load an Open File dialog box as seen in Figure 3-72.

Figure 3-72. RAL, File Open dialog window.

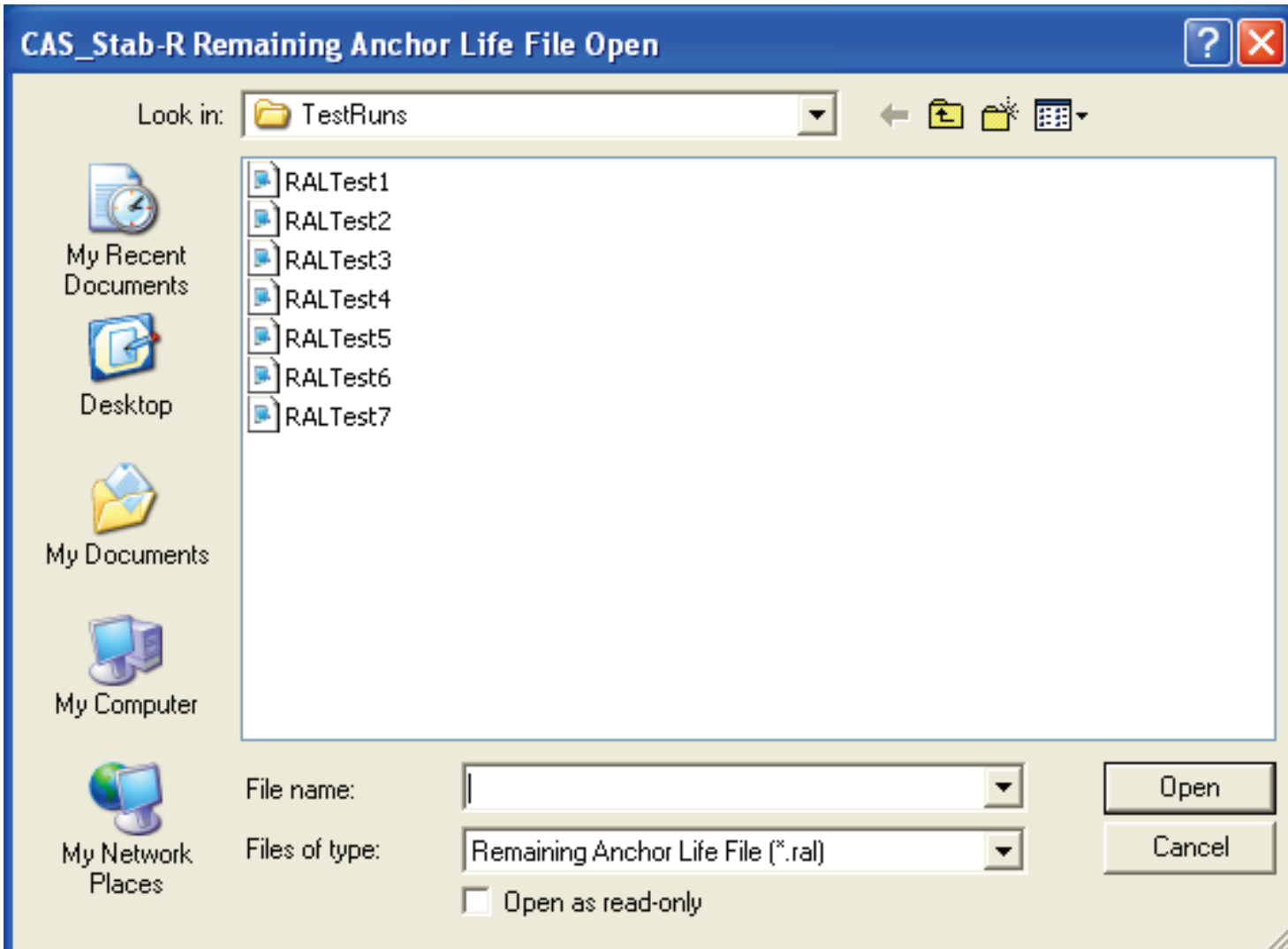

Select the file that holds the RAL information to read and click the Open button. The input and option controls will be populated with the loaded settings. The RAL files are stored as ASCII text. The format of the file is documented in Appendix B.

\subsubsection{File - Save}

Choose the File - Save option to write the current RAL configuration settings to the currently selected RAL file. If a file has not been previously opened, then a Save As operation is performed as described in the following sub-section 3.5.1.3. 


\subsubsection{File - Save As}

Choose the File - Save As option to save the current RAL configuration to a new file name. Clicking this option produces a File Save As dialog box as shown in Figure 3-73.

Figure 3-73. RAL File Save As dialog window.

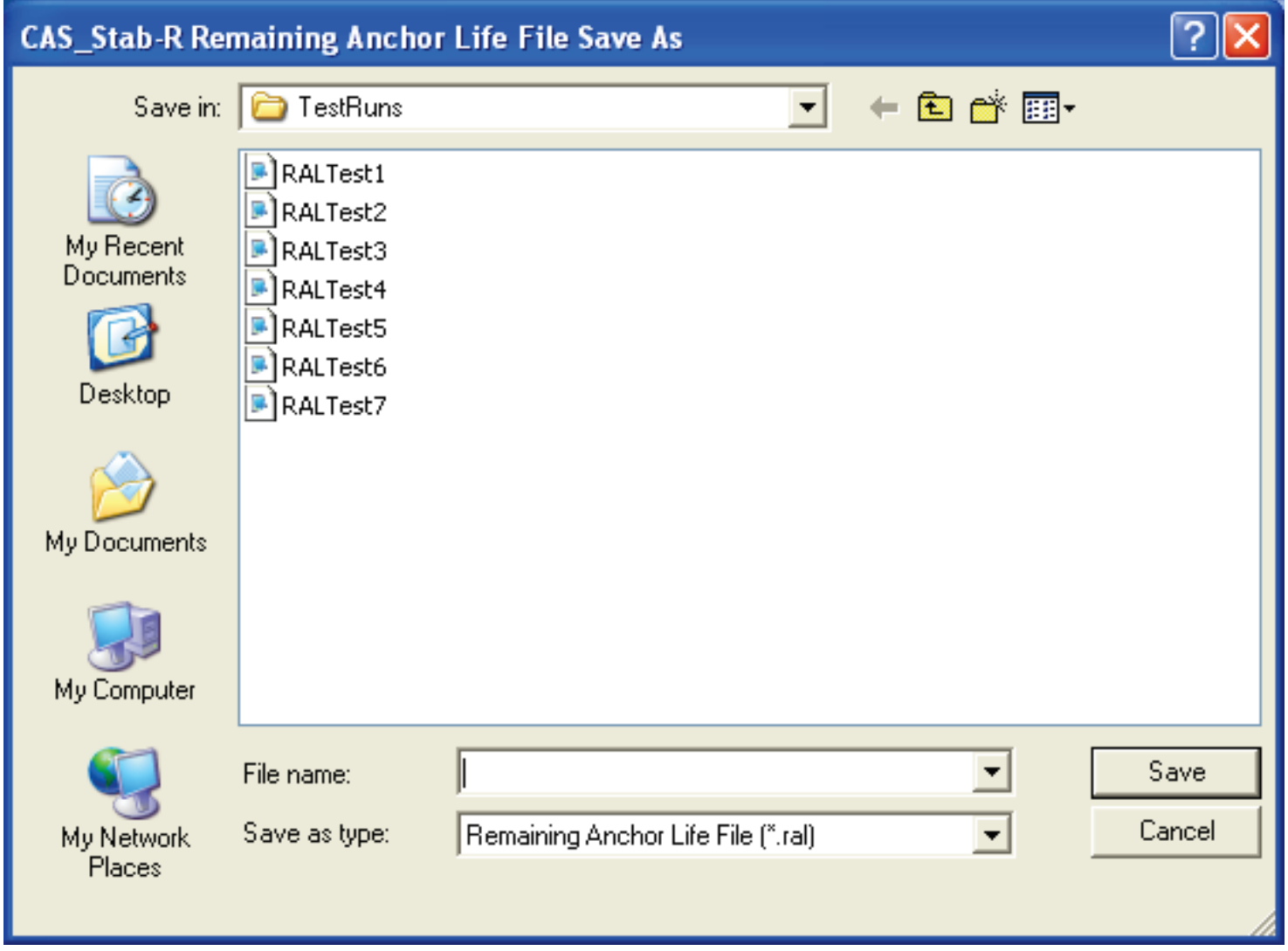

Specify the name of the file to store the current RAL configuration and click the Save button.

\subsubsection{File - Exit}

Choose the File - Exit option to close the RAL window. The CAS_Stab-R main screen shown in Figure 3-1 will be activated.

\subsubsection{Reduced Anchor Capacity Specification tab}

The Reduced Anchor Capacity Specification tab appears initially as shown in Figure 3-70. On this tab the user specifies the probability distributions for the variables, the method to determine the corroded cable size, and the reduced cable capacity curve for reduced capacity determination. The 
command button to create a Dakota-generated set of samples for the simulation is also on this tab. The various controls on this tab are divided into logical groupings and discussed in the following sections.

\subsubsection{Variables selection frame}

The variables that may be utilized in the simulation are shown in the highlighted frame shown in Figure 3-74. Two variables per simulation are available for selection. Selecting a variable allows the user to specify the distribution of values using the Selected Variable Distribution frame discussed in section 3.5.2.2. The initial lock-off force variable for the selected anchor cable is always enabled and available for selection. The second variable available is dependent on the corrosion method selected in the Select the Corrosion Method frame discussed in section 3.5.2.3.

Figure 3-74. Reduced Anchor Capacity Specification tab with Variables selection frame highlighted.

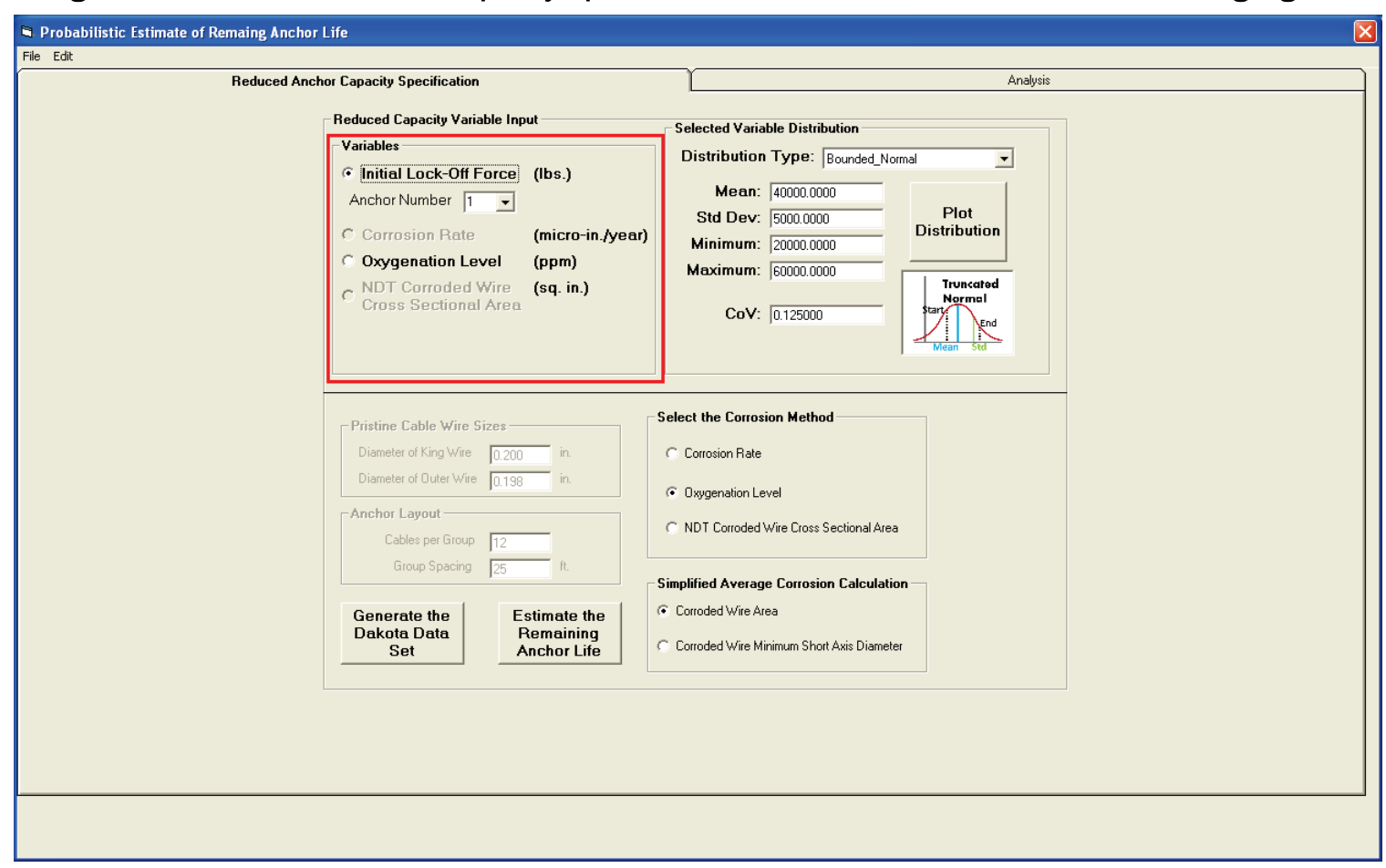

When a variable is selected, the values that define its sample distribution for the simulation will populate the input boxes in the Select Variable Distribution frame where they may be viewed and edited.

The Variables frame also contains a drop-down selection box labeled Anchor Number. It is located underneath the Initial Lock-Off Force variable. If a model containing more than one anchor is defined in the 
Analysis of Hydraulic Structure Stability Against Sliding portion of CAS_Stab-R, use the drop-down box to select the anchor lock-off force distribution to use in the simulation.

\subsubsection{Selected Variable Distribution frame}

Figure 3-75 shows the Selected Variable Distribution frame highlighted by a red box. This frame contains the input locations to specify the sample set distribution for the currently selected variable in the Variables frame.

Figure 3-75. Reduced Anchor Capacity Specification tab with highlighted Selected Variable Distribution frame.

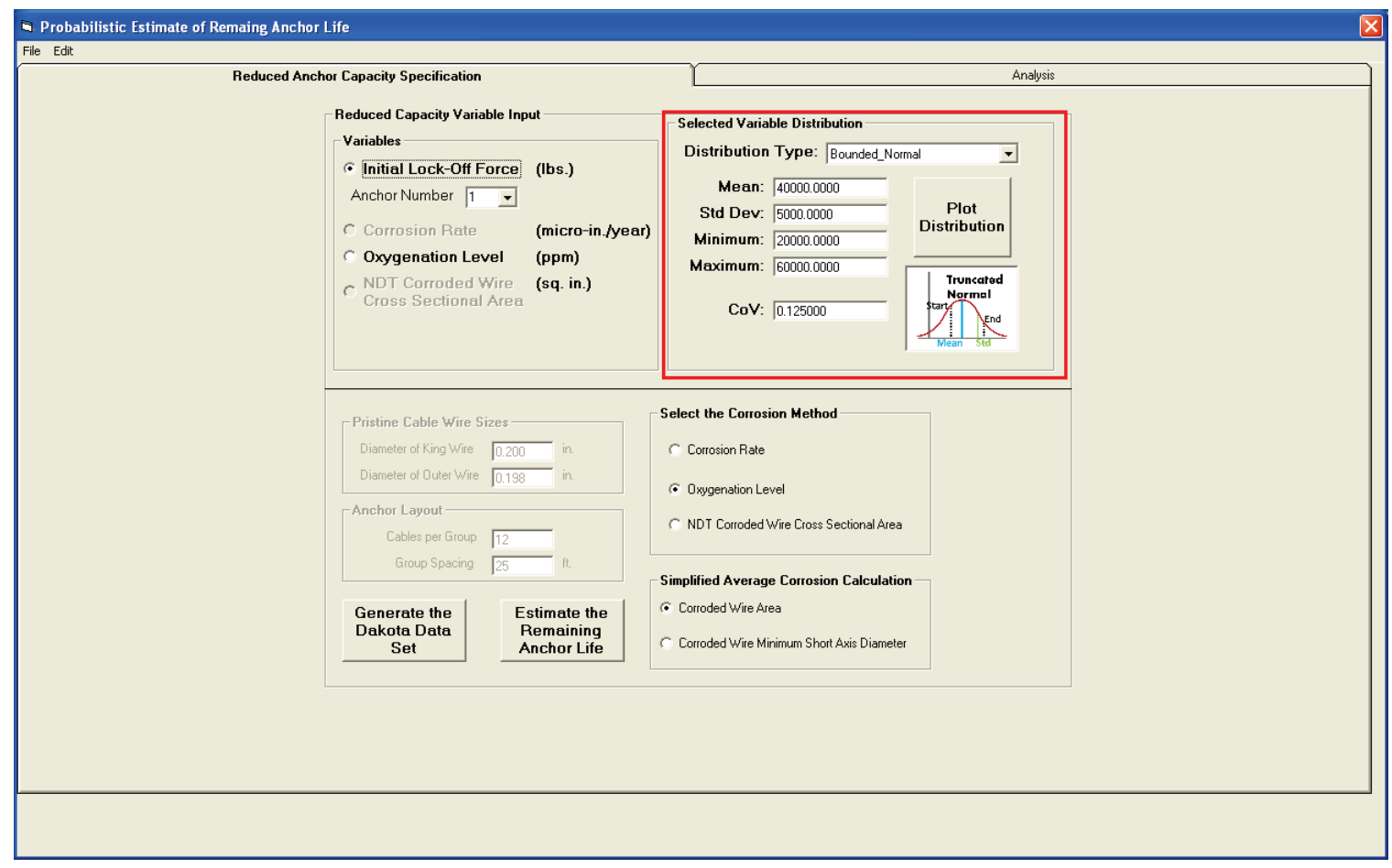

The drop-down box labeled Distribution Type allows the type of distribution to be selected. The options available are Bounded Normal, Bounded Log Normal, Uniform, and Triangular. The input boxes that allow editing of the parameters that define the distribution will change depending on the type of distribution chosen. As seen in Figure 3-75, a bounded normal distribution is defined by the mean, standard deviation, minimum, and maximum values. The coefficient of variation $(\mathrm{CoV})$ is updated each time a change is made to the mean or standard deviation. The CoV may also be entered by the user in which case the standard deviation value will be computed and updated accordingly. 
The bounded log normal distribution requires the same parameters as the bounded normal distribution. The uniform distribution is defined by the minimum and maximum value parameters. The triangular distribution is defined by minimum, mid-point, and maximum value parameters.

Clicking the Plot Distribution button will produce a probability density function plot of the distribution similar to that shown in Figure 3-10 located in section 3.4.2.2. That same section also discusses the method to view the CDF plot of the distribution similar to that shown in Figure 3-12.

\subsubsection{Select the Corrosion Method frame}

The frame labeled Select the Corrosion Method contains three option buttons as shown in the highlighted area of Figure 3-76. The option buttons allow the user to the select the method of determining a corrosion rate to compute the amount of corrosion experienced in the cable over time.

Figure 3-76. Reduced Anchor Capacity Specification tab with highlighted Select the Corrosion Method frame.

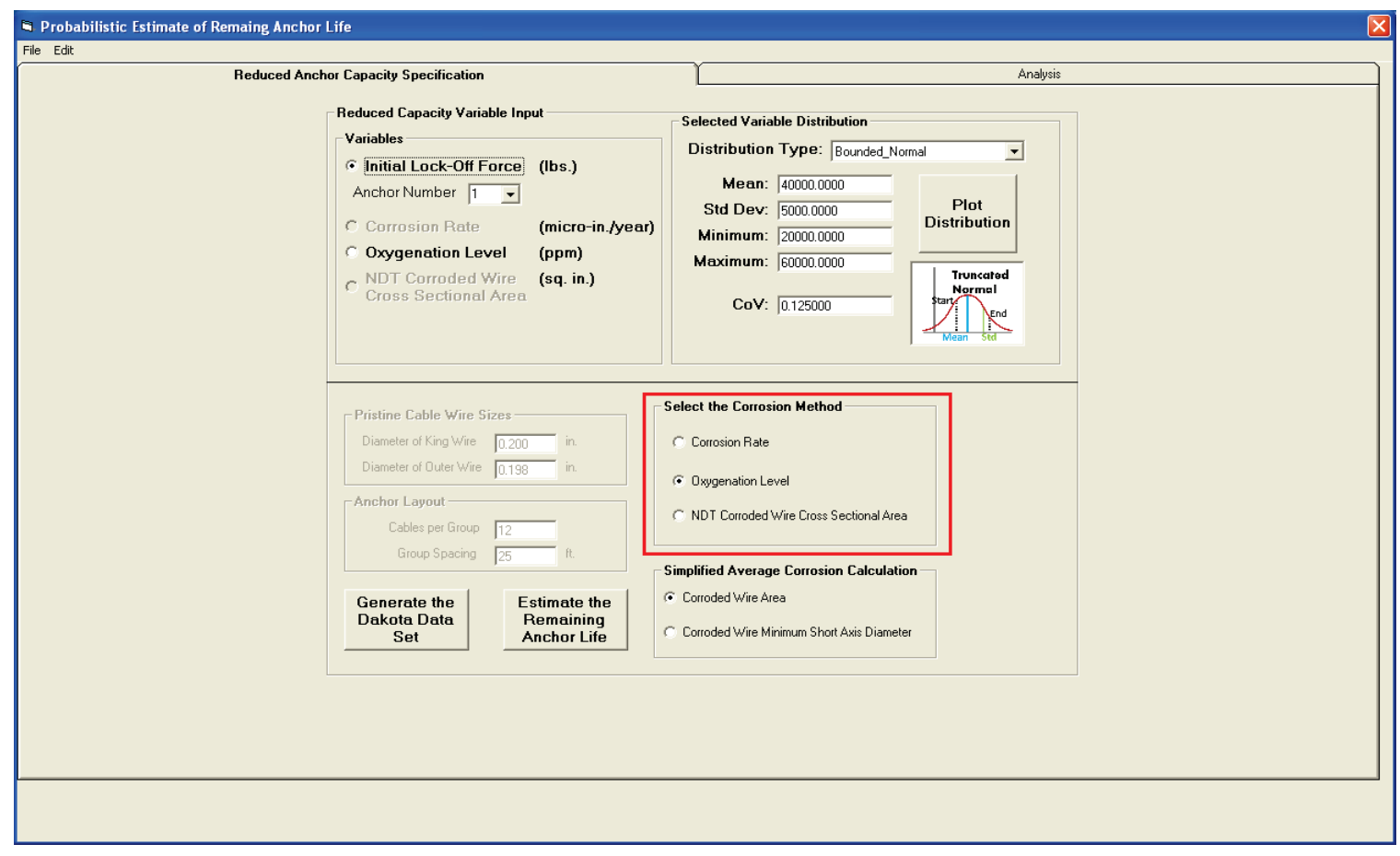

The first option is labeled Corrosion Rate. This selection is chosen to allow the user to specify a corrosion rate distribution in milli-inches/year or microns/year depending on the system of units in use. When this method is chosen, the corresponding option button in the Variables frame will be enabled. 
The second option is labeled Oxygenation Level. This selection is chosen to allow the user to specify the amount of oxygen in parts per million present in the environment surrounding the cable. The corrosion rate is computed by a scale factor derived from the red oxygenation curve seen in the righthand side of Figure 3-55. When this method is chosen, the corresponding option button in the Variables frame will be enabled.

The third option is labeled NDT Corroded Wire Cross Sectional Area. The option is chosen when the cross-sectional area of the cable has been determined by the use of a non-destructive means of testing. When this method is chosen, the corresponding option button in the Variables frame will be enabled.

\subsubsection{Simplified Average Corrosion Calculation frame}

The frame labeled Simplified Average Corrosion Calculation contains two option buttons as shown in the highlighted area of Figure 3-77. These options determine the scale factors to compute the reduced capacity of a corroded cable. The scale factors have been determined from statistical fits of the data points collected during pull tests of corroded cables conducted at the ERDC. The results of the pull tests and the details of the curve fits have been published in Ebeling et al. (2016). The curves are seen in Figures 3-78 and 3-79.

Figure 3-77. Reduced Anchor Capacity Specification tab with highlighted Simplified Average Corrosion Calculation frame.

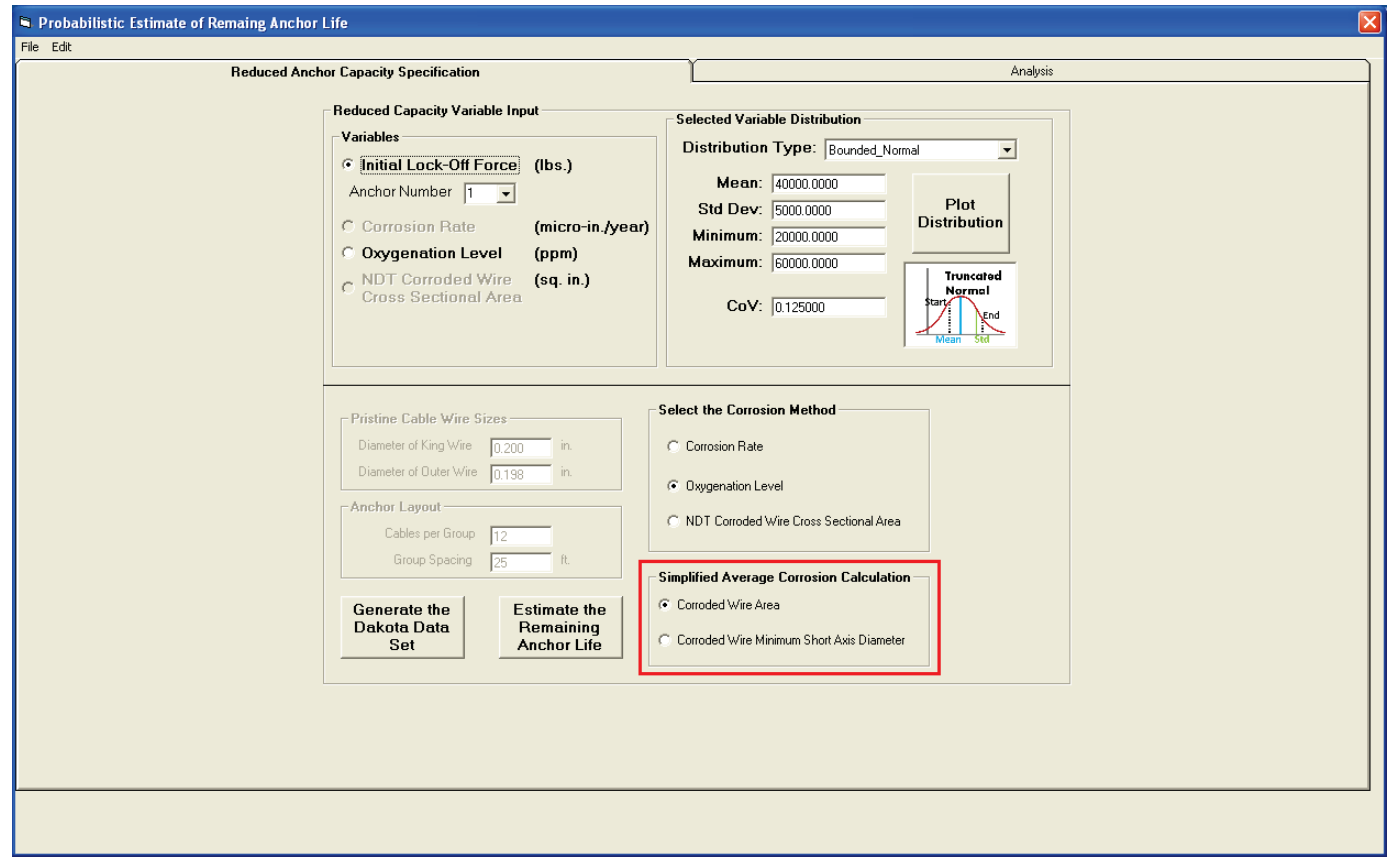


Figure 3-78. Cable failure forces and corresponding minimum corroded wire areas.

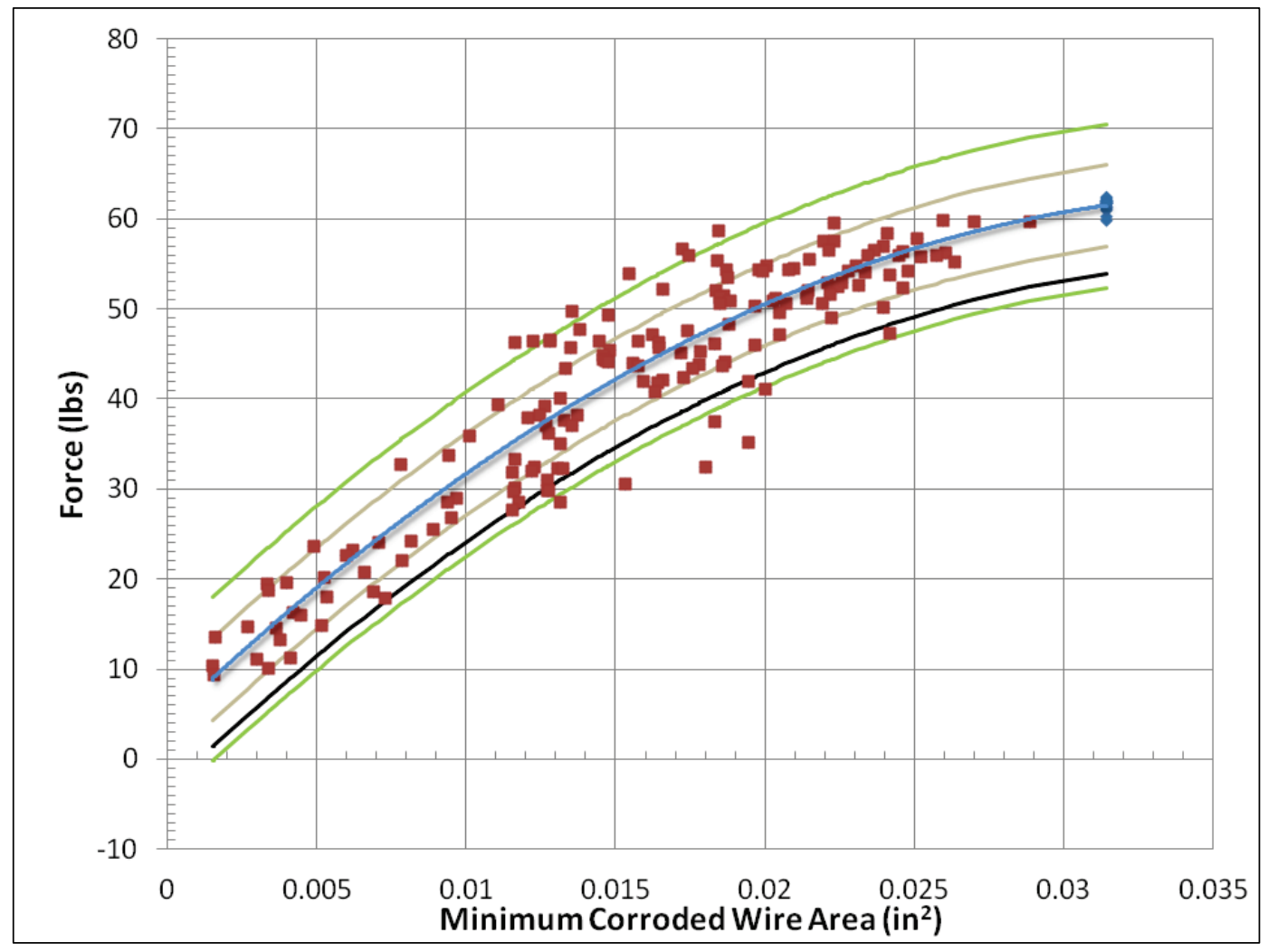

Figure 3-79. Cable failure forces and corresponding minimum short axis diameters.

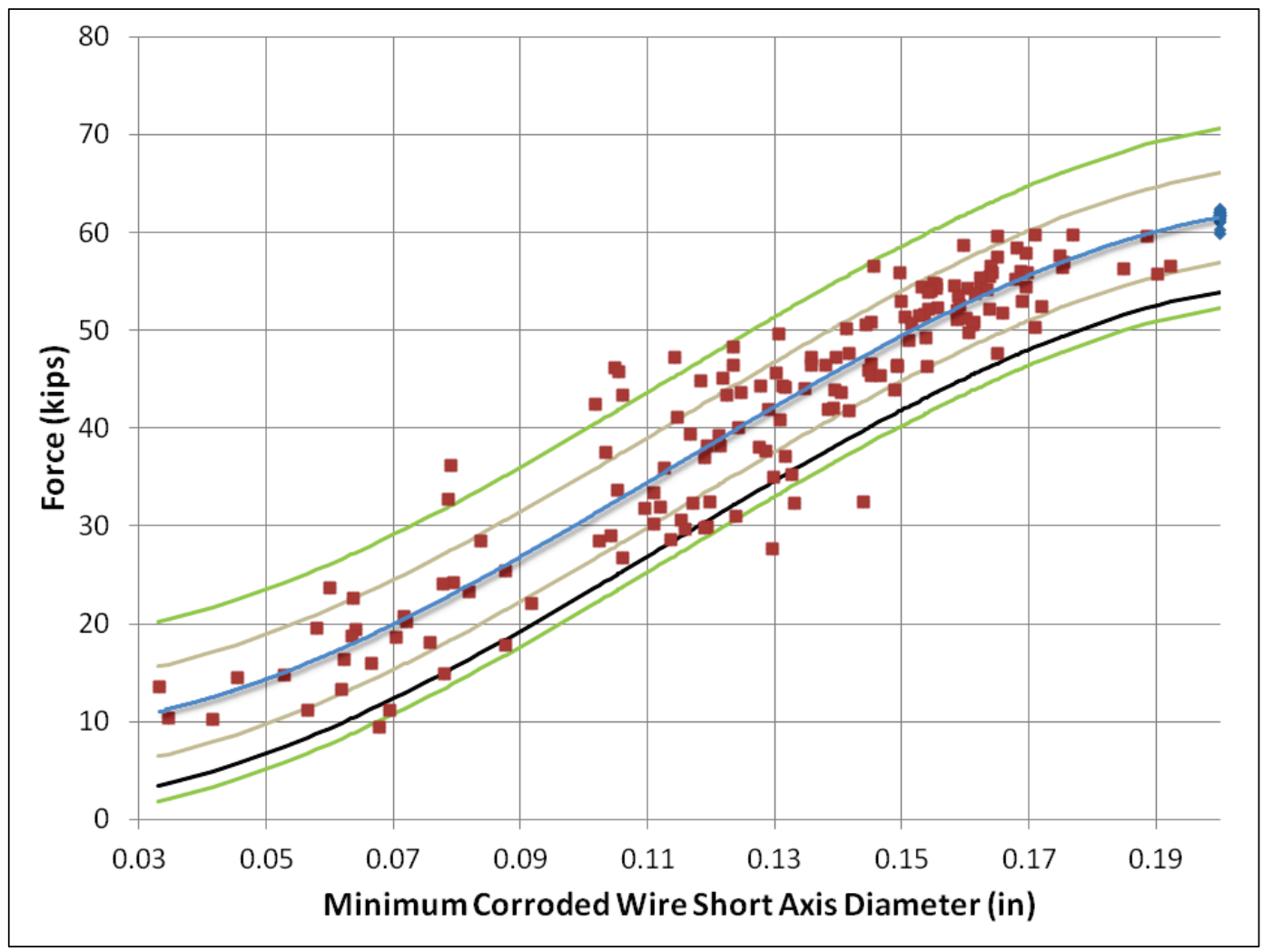


The first option is labeled Corroded Wire Area. Select this option to compute the corroded cable reduced capacity during the analysis phase using Equation 3-1. Equation 3-1 was derived from the curve fit for the data points shown in Figure 3-78 and corresponds to the blue line in the graph. Note that this set of coefficients is for use with the English system of units, and the area is given in square inches.

$$
R C=-43087028.19 * A^{2}+3176464.38 * A+4175.65
$$

where:

$$
\begin{aligned}
R C & =\text { Corroded Cable Reduced Capacity } \\
A & =\text { Corroded Cable Area }
\end{aligned}
$$

The second option is labeled Corroded Wire Minimum Short Axis Diameter. Select this option to compute the corroded cable reduced capacity during the analysis phase using Equation 3-2. Equation 3-2 was derived from the curve fit for the data points shown in Figure 3-79 and corresponds to the blue line in the graph. As noted above, this set of coefficients is for use with the English system of units, and the diameter is given in inches.

$$
R C=-12418355.96 * D^{3}+4237505.62 * D^{2}+-92773.55 * D+9918.59
$$

where:

$R C=$ Corroded Cable Reduced Capacity

$D=$ Corroded Cable Short Axis Diameter.

\subsubsection{Generate the Dakota Data Set button}

The button labeled Generate the Dakota Data Set is located in the lower left corner of this tab as shown in Figure 3-77. Use this button to generate a Dakota-generated simulation sample set for the selected variables utilizing their corresponding distributions. Clicking this button produces an input window for the user to enter the number of samples to generate as shown in Figure 3-80.

A set of samples will be generated for three variables. The first variable is the lock-off force applied to the cable. The second variable is determined by the currently selected option in the Select the Corrosion Method frame. The third variable is referred to as the dispersion variable. This variable is utilized because during the analysis the reduced anchor capacity calculation will always generate a value that lies on the mean (blue) curve in 
Figure 3-78 or Figure 3-79. The dispersion variable is assigned a mean value of 0 , a standard deviation of 1 , minimum value of -3 , and maximum value of 3. During the analysis, the value of the dispersion variable is multiplied by the standard deviation for the selected curve and then added to the reduced anchor capacity value to yield a variable reduced anchor capacity. The variable reduced anchor capacity will have a standard deviation equal to the standard deviation of the chosen reduced capacity curve, minimum value of -3 times the standard deviation, and maximum value of 3 times the standard deviation. The probability distribution function (PDF) curve for this distribution is shown in Figure 3-81.

Figure 3-80. Number of Simulations input box.

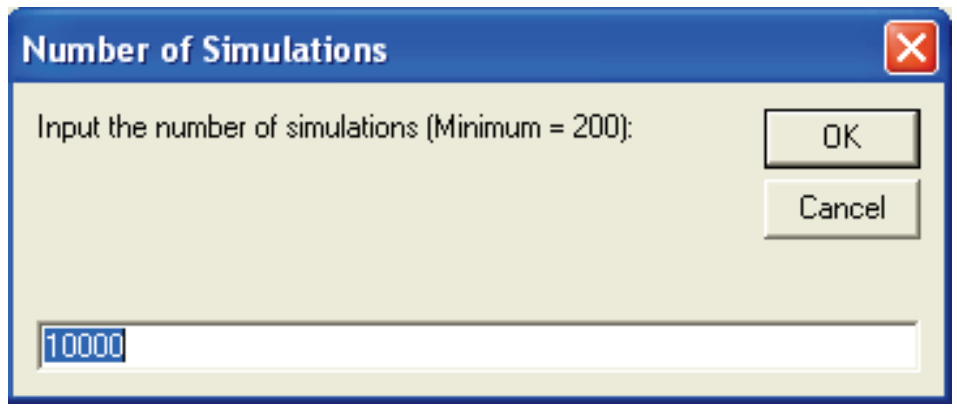

Figure 3-81. Dispersion Variable PDF curve.

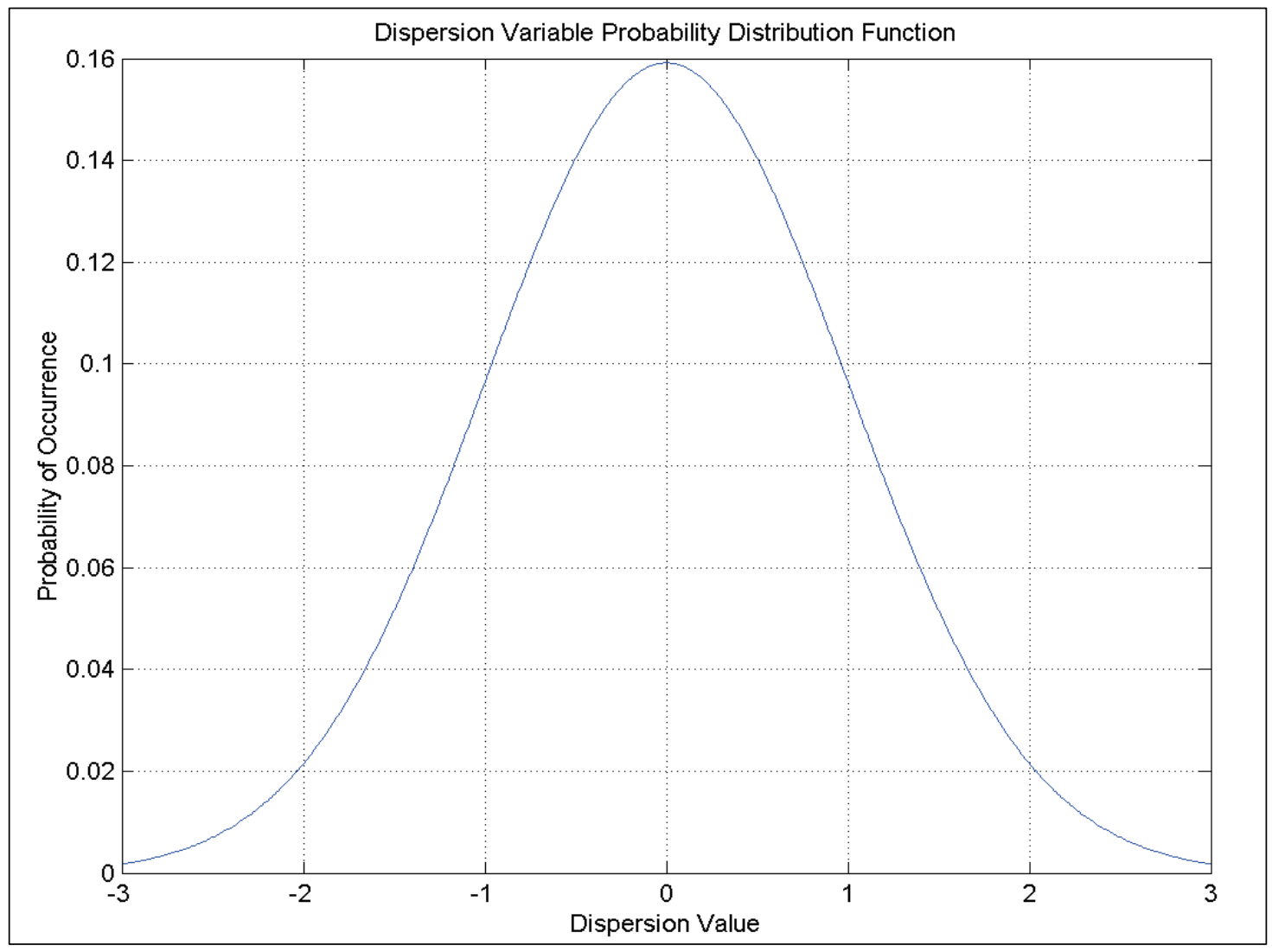




\subsubsection{Estimate the Remaining Anchor Life button}

In the lower left corner of this tab is the Estimate the Remaining Anchor Life button. It is positioned to the immediate right of the Generate the Dakota Data Set button. When the RAL window is activated, this button will be disabled as seen in Figure 3-70. To enable the button, a Dakota data set must be loaded into memory. This is accomplished by using the Generate a Dakota Data Set button to generate a new Dakota data set or by loading a previously saved RAL file. When the user clicks this button, the Analysis tab is activated, and the program will perform a TTF analysis and plot a TTF histogram. The Analysis tab is discussed in the following section.

\subsubsection{Analysis tab}

The Analysis tab is available for use following a click of the Estimate the Remaining Anchor Life button on the Reduced Anchor Capacity Specification tab. When the tab is loaded, the window will appear as shown in Figure 3-82. (The red Display Area does not appear. It is added here as a label.)

Figure 3-82. Initial RAL Analysis tab.

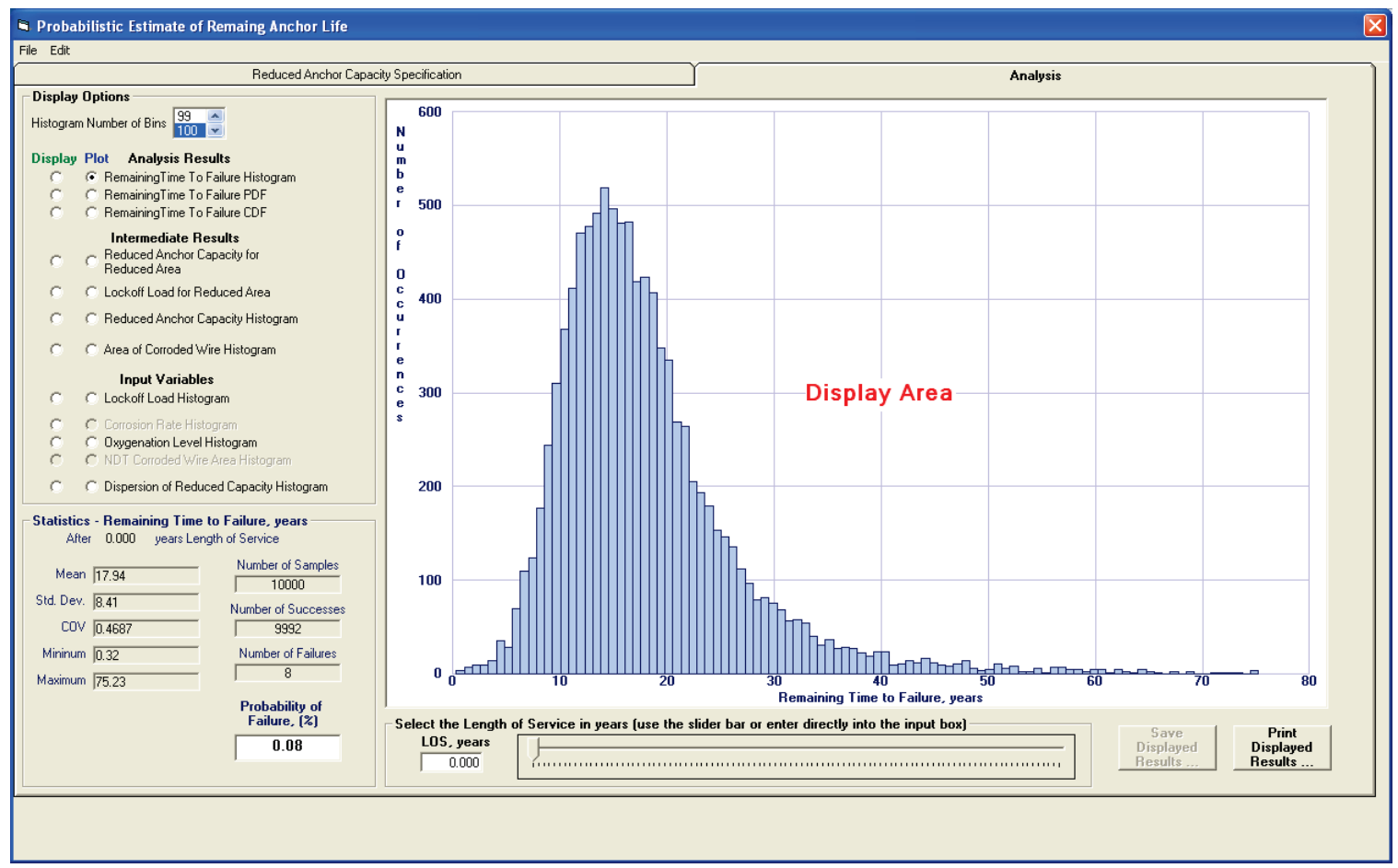


The most visible feature of the Analysis tab is the Display Area. Histogram charts, scatter plots, and tabular data are presented to the user in this area. The remaining controls on the Analysis tab are contained in the Display Options frame, Statistics frame, and LOS frame. The tab also contains Save Displayed Results and Print Displayed Results buttons. These controls are discussed in the following sections.

\subsubsection{Display Options frame}

The Display Options frame provides controls to select the number of bins for histogram plots and to select the data for display in the Display Area. To select the number of bins for histogram plots, use the mouse to scroll to the desired number of bins to plot. Click on the desired number. If a histogram chart is currently displayed, it will be updated with the newly specified number of bins.

The remaining controls in this frame are option buttons to choose the data to view in the Display Area. The data may be viewed in graphical or tabular form. To view graphical data, choose an option button in the column labeled Plot. To view tabular data, choose an option button in the column labeled Display.

The data available for display is separated into three categories: Analysis Results, Intermediate Results, and Input Variables. The data in the Analysis Results and Intermediate Results categories will vary according to the LOS selected for the cable in the Length of Service frame. The data in the Input Variables remain constant regardless of the LOS.

The data available in the Analysis Results category are the following:

- the Remaining Time to Failure histogram

- the Remaining Time to Failure PDF curve

- the Remaining Time to Failure CDF curve.

The data available in the Intermediate Results category are the following:

- a scatter plot of the Reduced Anchor Capacity for the Reduced Area (if the Simplified Corrosion Calculation Method option was the Corroded Wire Area) or the Reduced Diameter (if the Simplified Corrosion Calculation Method option was the Corroded Wire Short Axis Diameter) 
- a scatter plot of the Lock-off Load for the Reduced Area (if the Simplified Corrosion Calculation Method option was the Corroded Wire Area) or the Reduced Diameter (if the Simplified Corrosion Calculation Method option was the Corroded Wire Short Axis Diameter)

- a scatter plot of the Reduced Anchor Capacity Histogram

- either the Reduced Area Histogram or Reduced Diameter Histogram (again dependent on the Simplified Corrosion Calculation Method chosen).

The data available in the Input Variables category are the following:

- the Lock-off Load histogram

- the Corrosion values histogram (only enabled if this is the Corrosion Method selected on the Reduced Anchor Capacity Specification tab)

- the Oxygenation levels histogram (only enabled if this is the Corrosion Method selected on the Reduced Anchor Capacity Specification tab)

- the NDT area histogram (only enabled if this is the Corrosion Method selected on the Reduced Anchor Capacity Specification tab)

- the Reduced Capacity Dispersion Variable histogram.

\section{Remaining Time To Failure Histogram}

Clicking the Remaining Time To Failure Histogram button in the Plot column produces a histogram plot as seen in Figure 3-82. The remaining time to failure is computed for each sample in the simulation in the following manner:

1. The reduced cable diameter is the pristine cable diameter minus the product of the corrosion rate and the length of service time.

2. The cable diameter required to provide the capacity for the lock-off load is obtained by solving for $x$ in Equation 3.1 or Equation 3.2. Which equation is determined by the Simplified Average Corrosion Calculation option selection, either area or diameter.

3. The Time To Failure is equal to the difference of the reduced cable diameter and the lock-off load diameter divided by the corrosion rate. If the reduced cable diameter is less than the lock-off load diameter, the Time To Failure is set to 0 . 
The histogram data is generated from the non-zero TTF samples.

Therefore the data presented are the remaining TTF for the number of samples that remain intact after the selected Length of Service time.

Clicking the Remaining Time To Failure Histogram button in the Display column produces a tabular printout of the histogram data points along with the individual TTF samples in the Display Area. Figure 3-83 shows the tabular data that corresponds to the histogram plot in Figure 3-82. The user may use the scroll bar to access all the data points.

Statistical values for the displayed data are viewed in the Statistics frame below the Display Options frame. Displayed data can be saved or printed using the buttons located at the bottom right of the window.

Figure 3-83. Remaining Time To Failure Histogram display.

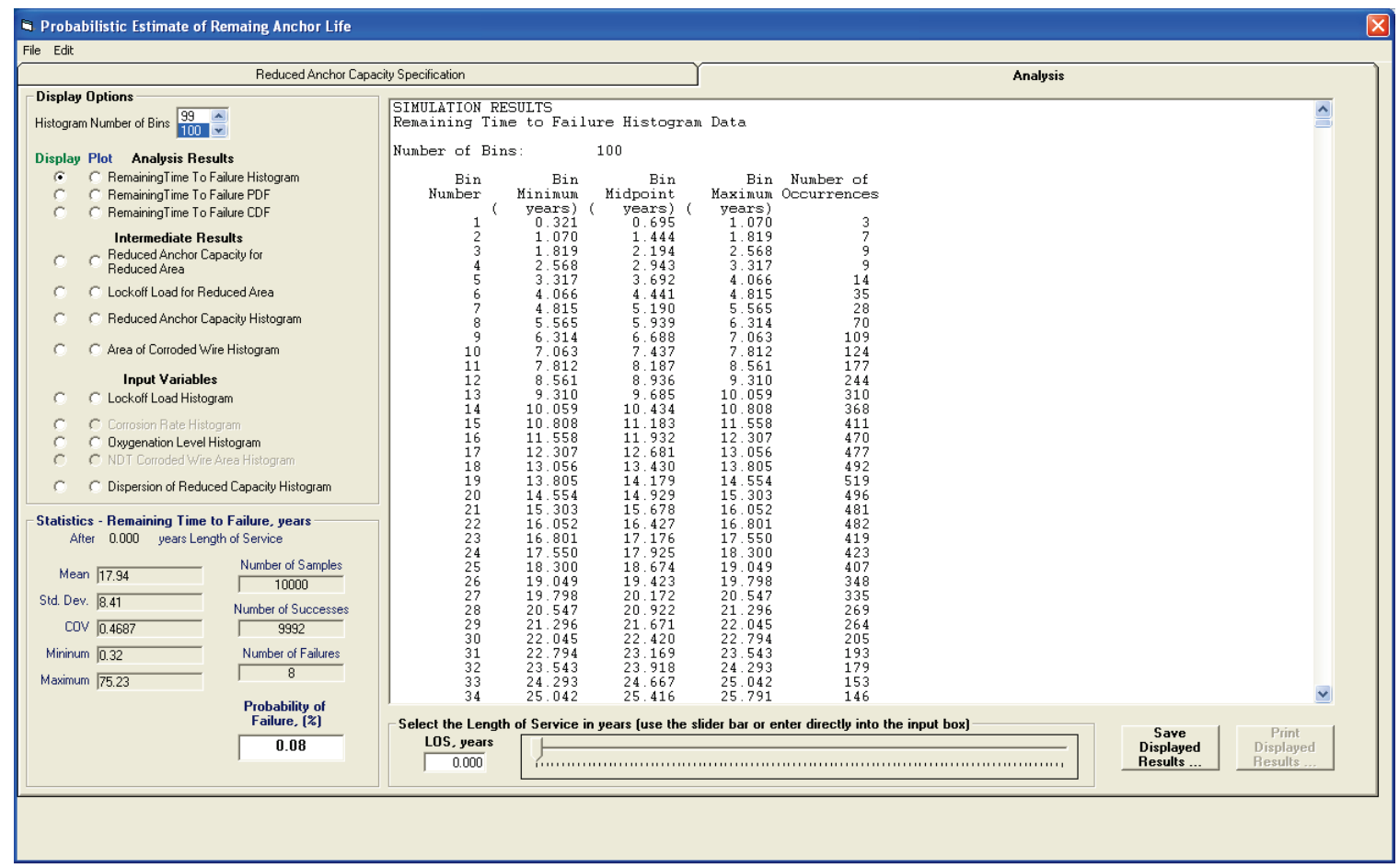

\section{Remaining Time To Failure PDF}

The PDF of the remaining TTF data can be plotted by clicking the Remaining Time To Failure PDF option button in the Plot column. The PDF data is computed for a 100-year time period following the selected LOS time. An example plot is shown in Figure 3-84. 
Figure 3-84. Remaining Time To Failure PDF plot.

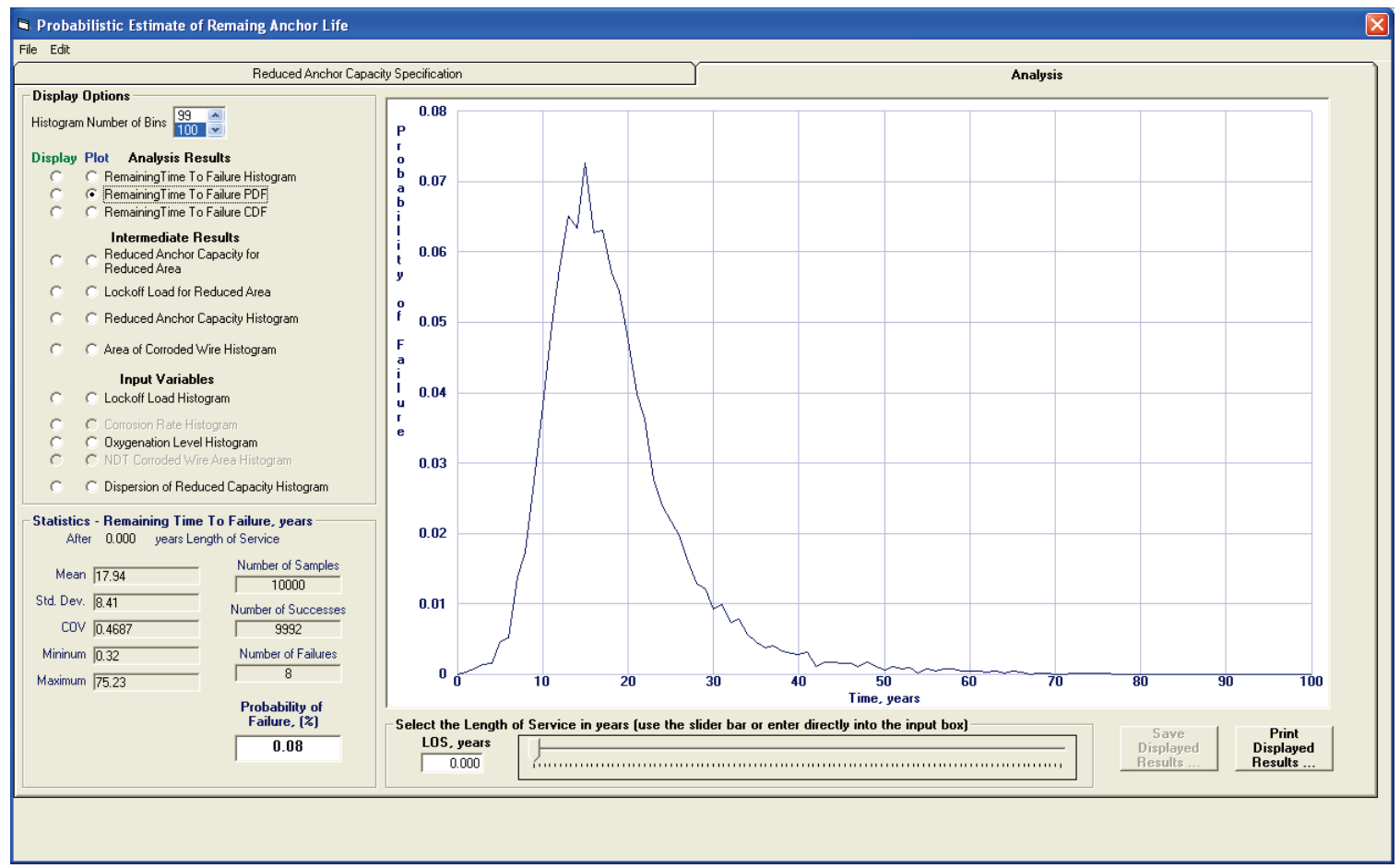

The PDF data of the remaining TTF can be displayed by clicking the Remaining Time To Failure PDF option button in the Display column. A sample PDF data display is shown in Figure 3-85. The data in Figure 3-85 correspond to the plot in Figure 3-84. A scroll bar is present in the display to allow access to all data points.

\section{Remaining Time To Failure CDF}

The CDF of the remaining TTF data can be plotted by clicking the Remaining Time To Failure CDF option button in the Plot column. The $\mathrm{CDF}$ data are computed for a 100-year time period following the selected LOS time. The CDF plot that corresponds to the PDF plot in Figure 3-84 is shown in Figure 3-86.

The CDF data of the remaining TTF can be displayed by clicking the Remaining Time To Failure CDF option button in the Display column. The format of the CDF data display matches that of the PDF display as shown in Figure 3-85. 
Figure 3-85. Remaining Time To Failure PDF display.

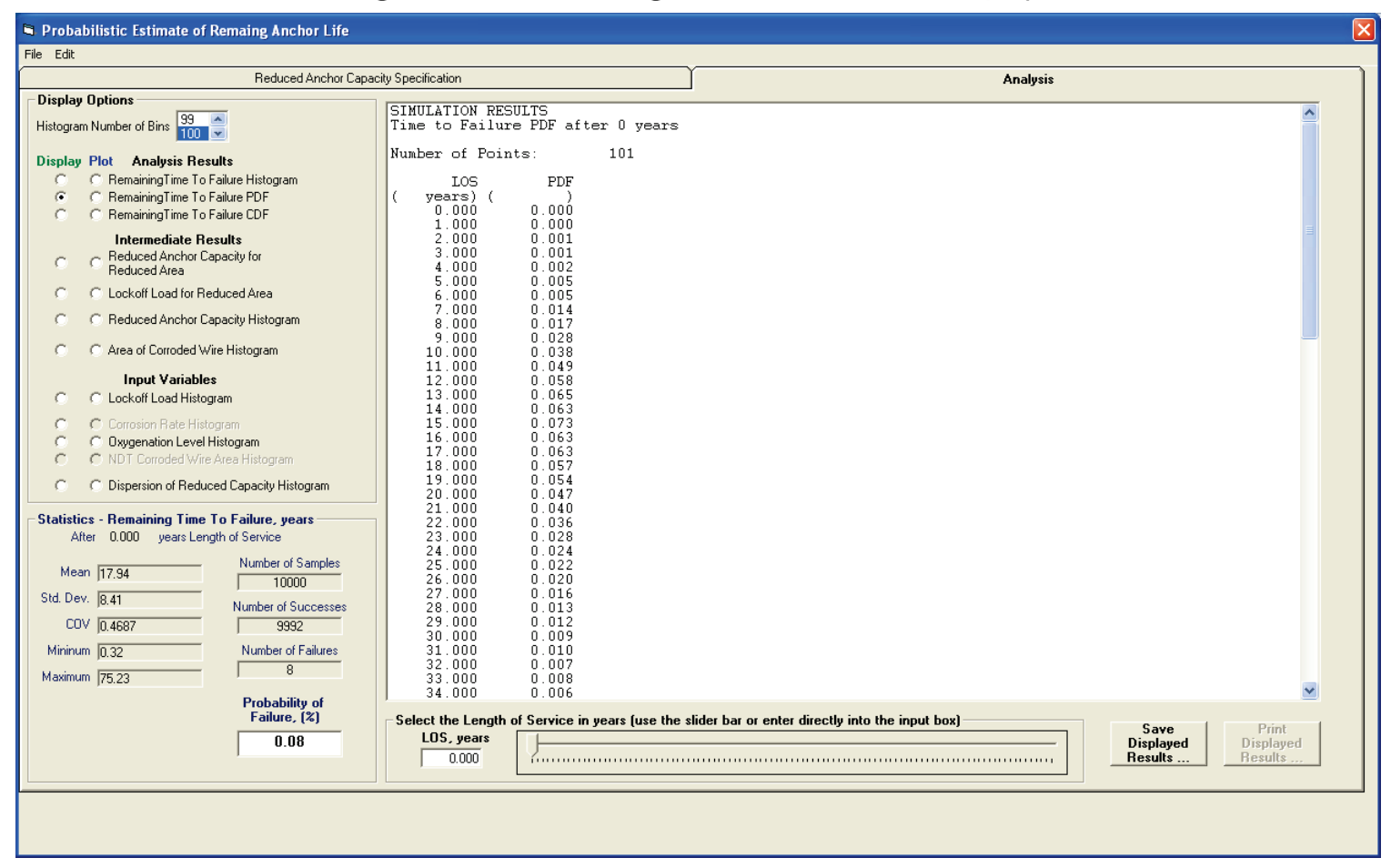

Figure 3-86. Remaining Time To Failure CDF display.

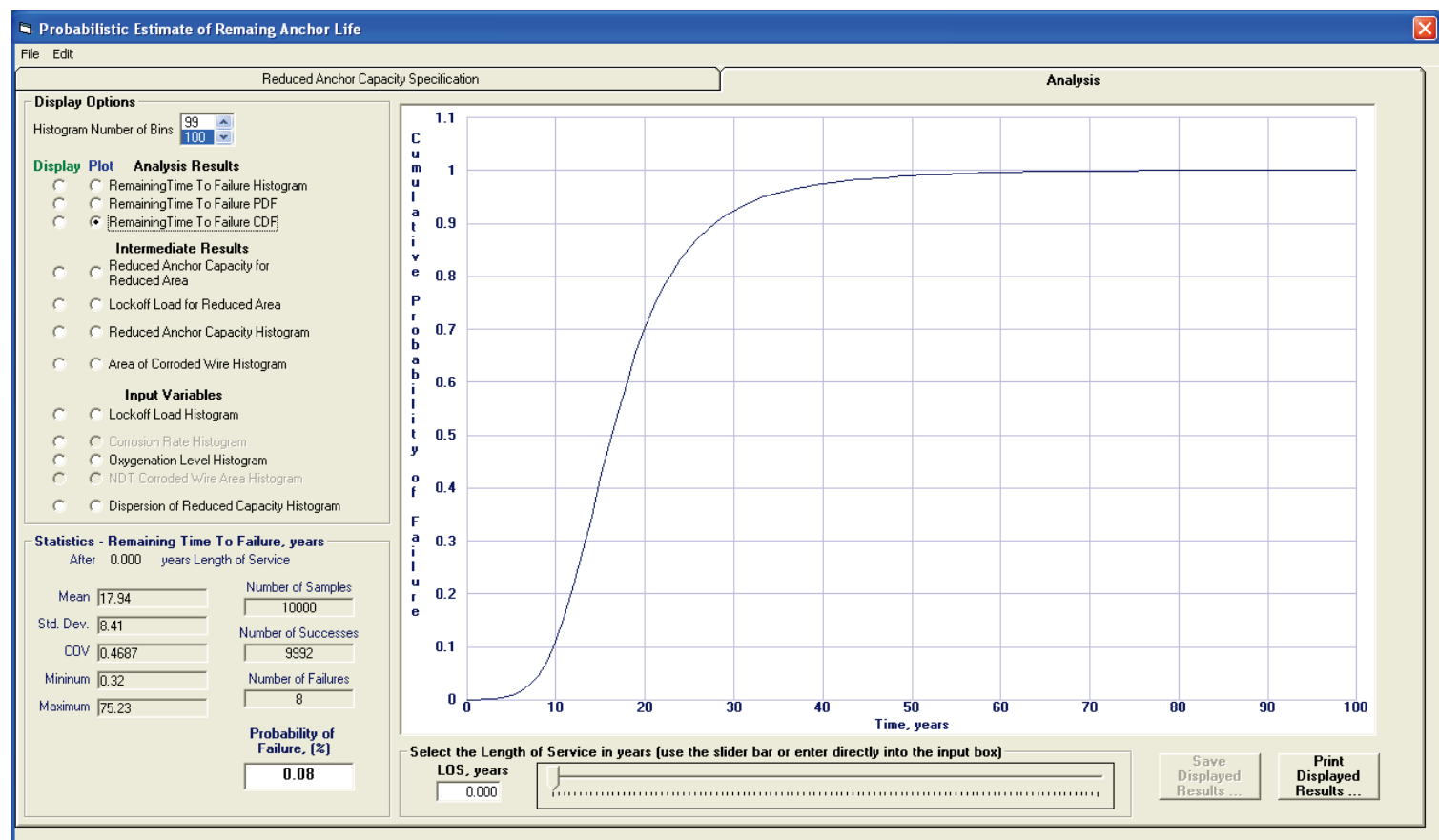




\section{Reduced Anchor Capacity for Reduced Area (Reduced Short Axis Diameter)}

A scatter plot of the Reduced Anchor Capacity for Reduced Area (Reduced Short Axis Diameter) can be viewed by clicking the corresponding option button in the Plot column. Whether this is reduced area data or reduced short axis diameter data is determined by the selected Simplified Average Corrosion Calculation option on the Reduced Anchor Capacity Specification tab. An example plot is shown in Figure 3-87.

Figure 3-87. Reduced Anchor Capacity for Reduced Area plot.

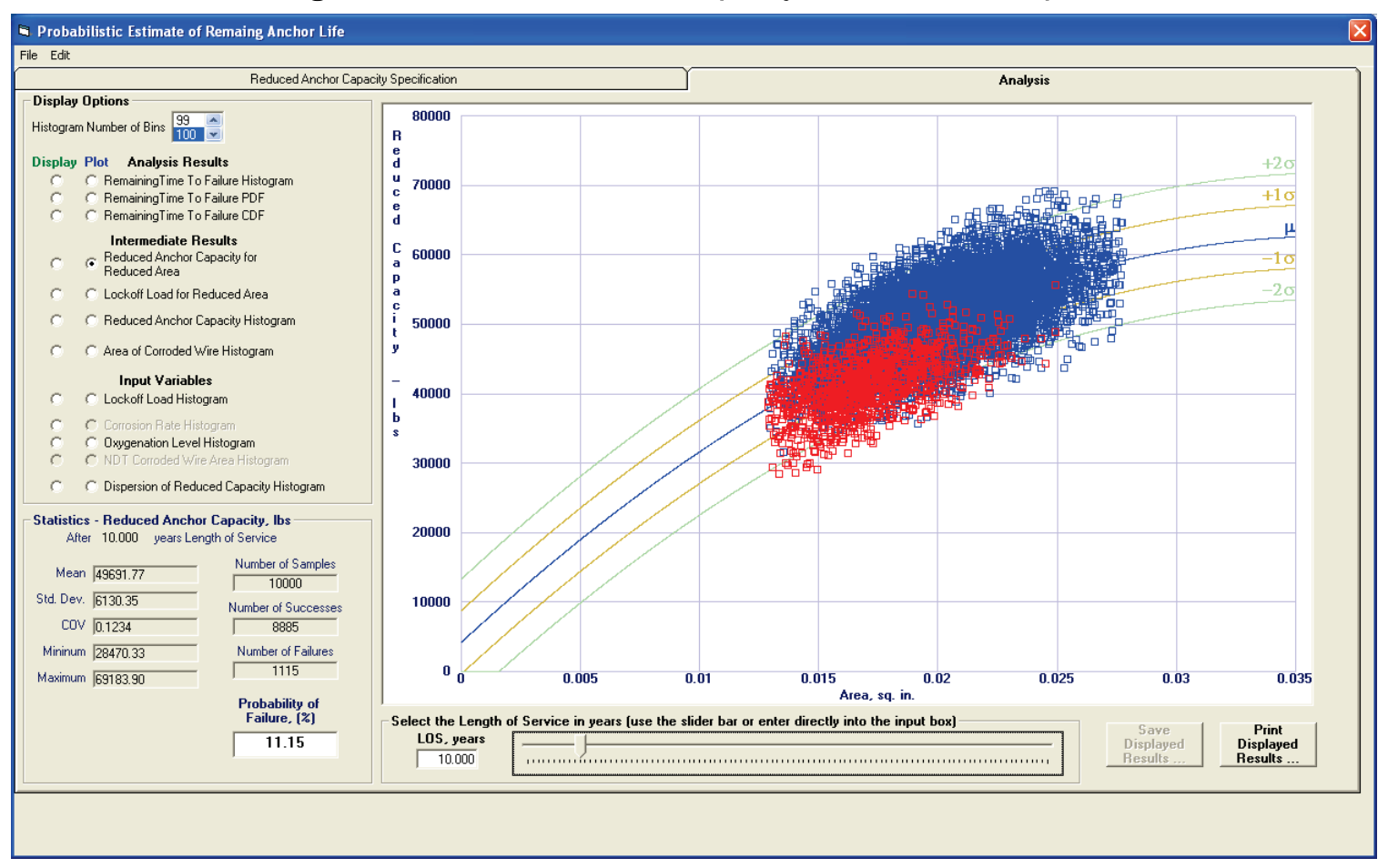

In this graph, a point is plotted for each sample in the data set. The $x$-coordinate of the point is the area of the corroded cable after the length of service time. The $y$-coordinate of the point is the reduced capacity of the corroded cable. Blue points indicate the reduced capacity exceeded the lock-off load value for the sample, so the cable has not reached failure. Red points indicate the reduced capacity is less than the lock-off load value, so the cable has reached the failure point for that sample.

Clicking the option button in the Display column for this graph produces a tabular list of the reduced anchor capacity, the corroded cable diameter, the lock-off load, and an indication if the sample resulted in a cable failure. The beginning of the list for the data in Figure 3-87 is shown in Figure 3-88. 
Figure 3-88. Reduced Anchor Capacity for Reduced Area display.

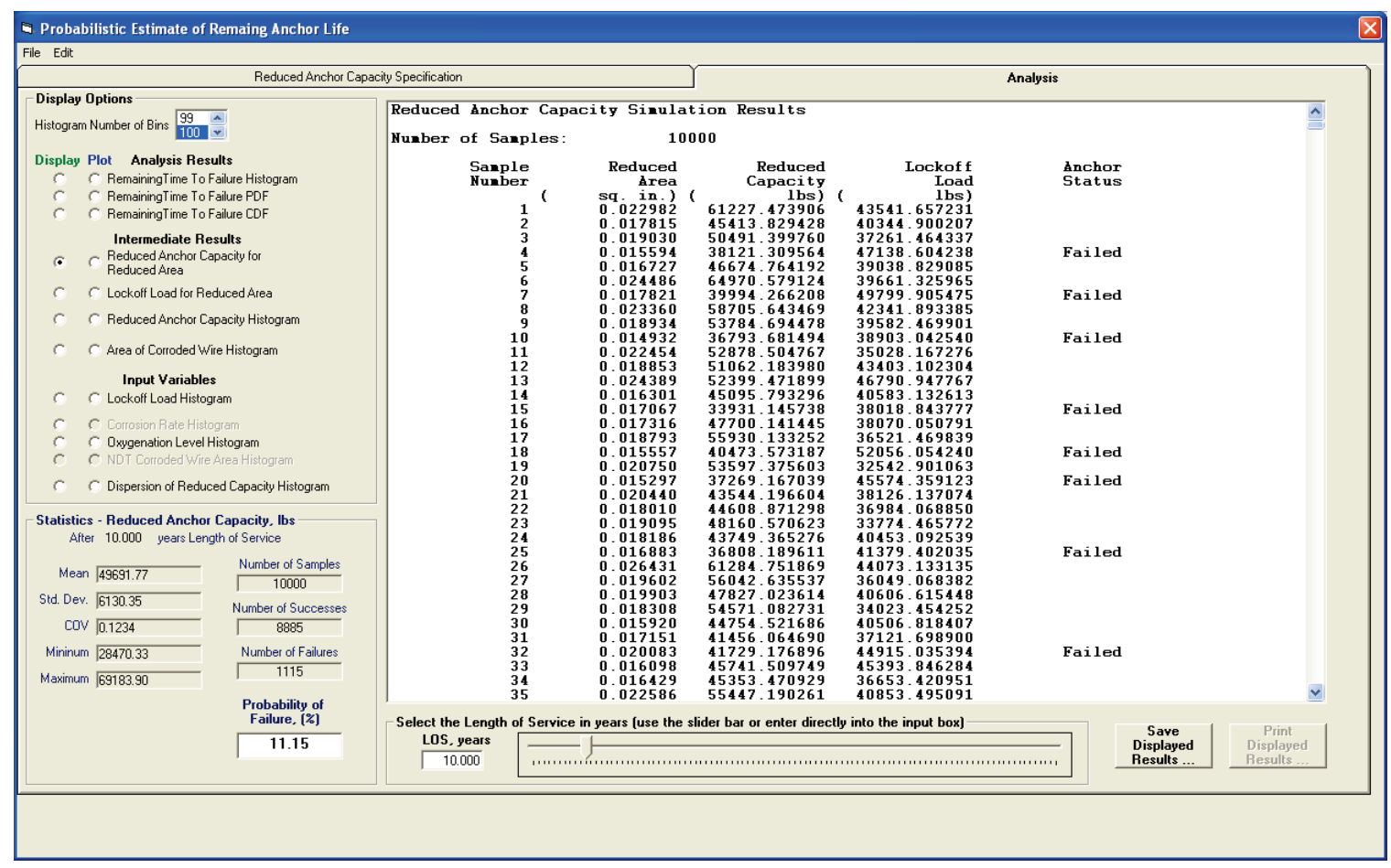

\section{Lock-off Load for Reduced Area (Reduced Short Axis Diameter)}

A scatter plot of the Lock-off Load for Reduced Area (Reduced Short Axis Diameter) can be viewed by clicking the corresponding option button in the Plot column. Whether this is reduced area data or reduced short axis diameter data is determined by the selected Simplified Average Corrosion Calculation option on the Reduced Anchor Capacity Specification tab. An example plot is shown in Figure 3-89.

In this graph a point is plotted for each sample in the data set. The $x$-coordinate of the point is the area of the corroded cable after the length of service time. The $y$-coordinate of the point is the lock-off load applied to the cable. Blue points indicate the reduced capacity exceeded the lock-off load value for the sample, so the cable has not reached failure. Red points indicate the reduced capacity is less than the lock-off load value, so the cable has reached the failure point for that sample.

Clicking the option button in the Display column for this graph produces the tabular list of the reduced anchor capacity, the corroded cable diameter, the lock-off load, and an indication if the sample resulted in a cable failure exactly as shown in Figure 3-88. Both the Reduced Anchor Capacity for Reduced Area (Short Axis Diameter) and the Lock-off Load for Reduced Area (Short Axis Diameter) utilize the same variables to form the graph. 
Figure 3-89. Lock-off Load for Reduced Area plot.

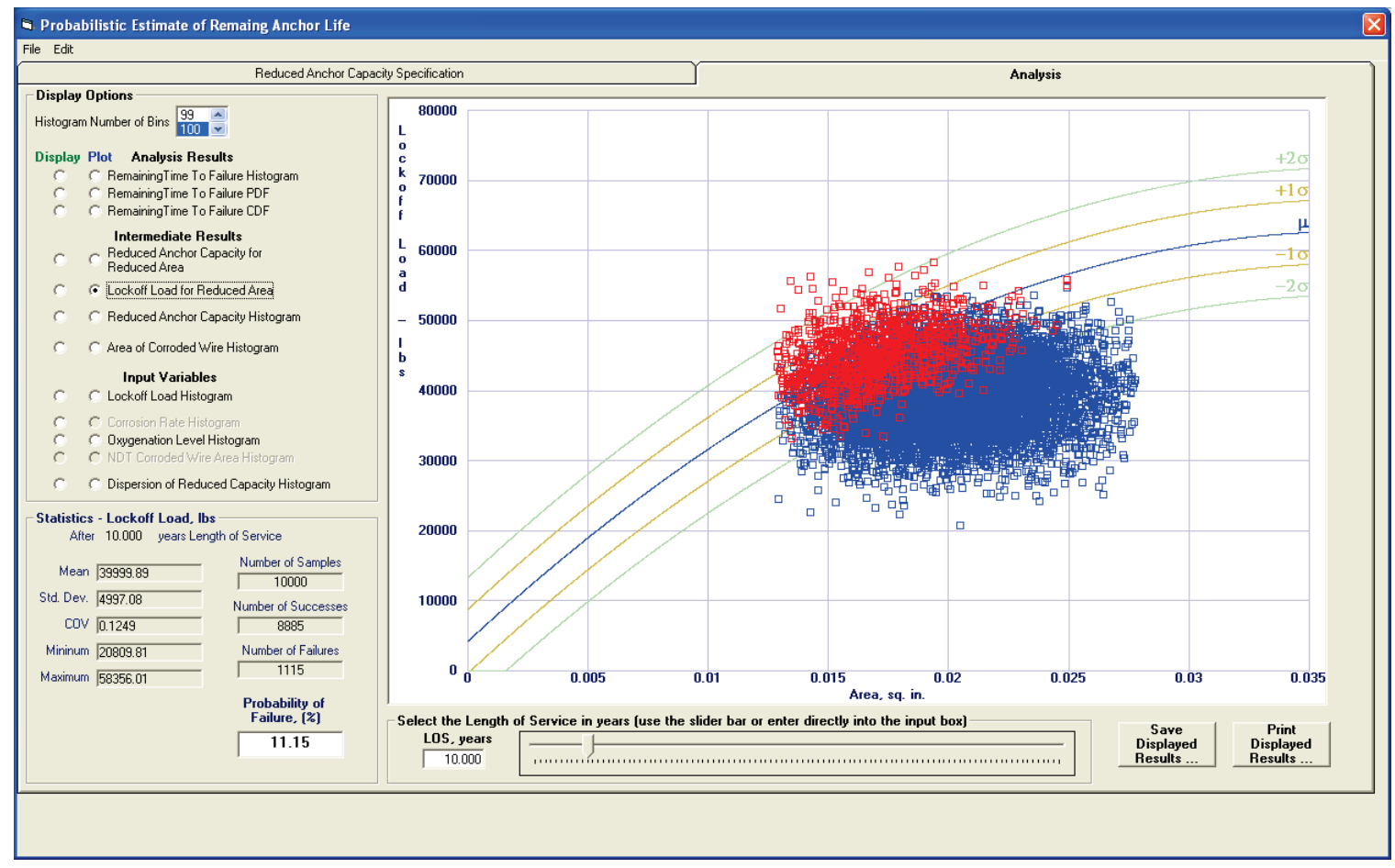

\section{Reduced Anchor Capacity Histogram}

Choose the Reduced Anchor Capacity Histogram option button in the Plot column to produce a histogram plot of the reduced anchor capacities due to corrosion. An example histogram of the reduced anchor capacities that were used in Figures 3-87 and 3-88 is shown in Figure 3-90.

\section{Area of Corroded Wire Histogram}

Select the Area of Corroded Wire Histogram option button in the Plot column to produce a histogram of the corroded wire area samples in the Display Area. An example corroded wire area histogram is shown in Figure 3-91. Select the Area of Corroded Wire Histogram option button in the Display column to produce a tabular list in the Display Area of the corroded wire area histogram values. The list will have the same format as that in Figure 3-83.

Selecting the corresponding option button in the Display column will produce a tabular list of the histogram data with the same format as seen in Figure 3-83. The second line in the list will reflect that this list of values is for the reduced anchor capacity. 
Figure 3-90. Reduced Anchor Capacity Histogram.

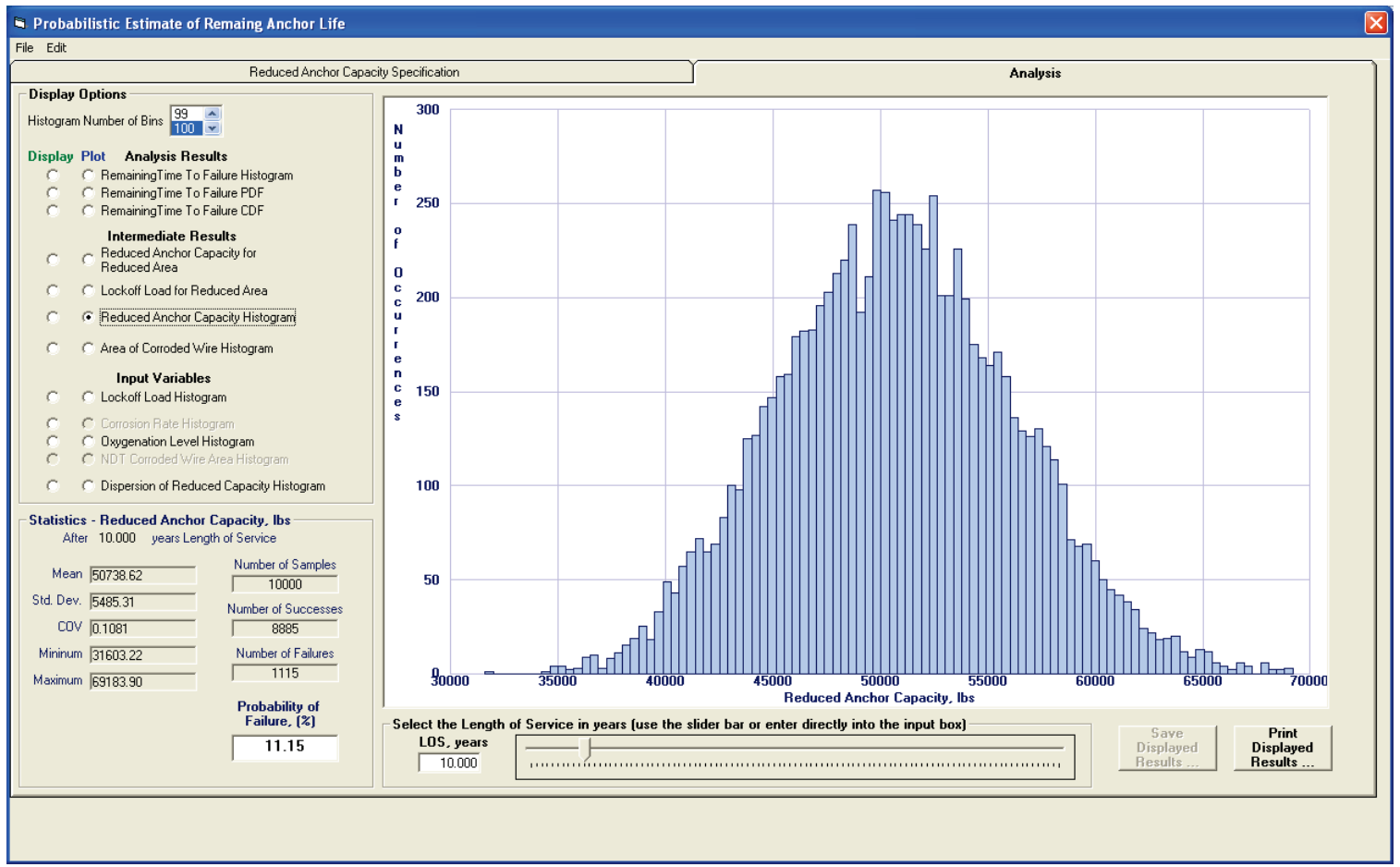

Figure 3-91. Area of Corroded Wire Histogram.

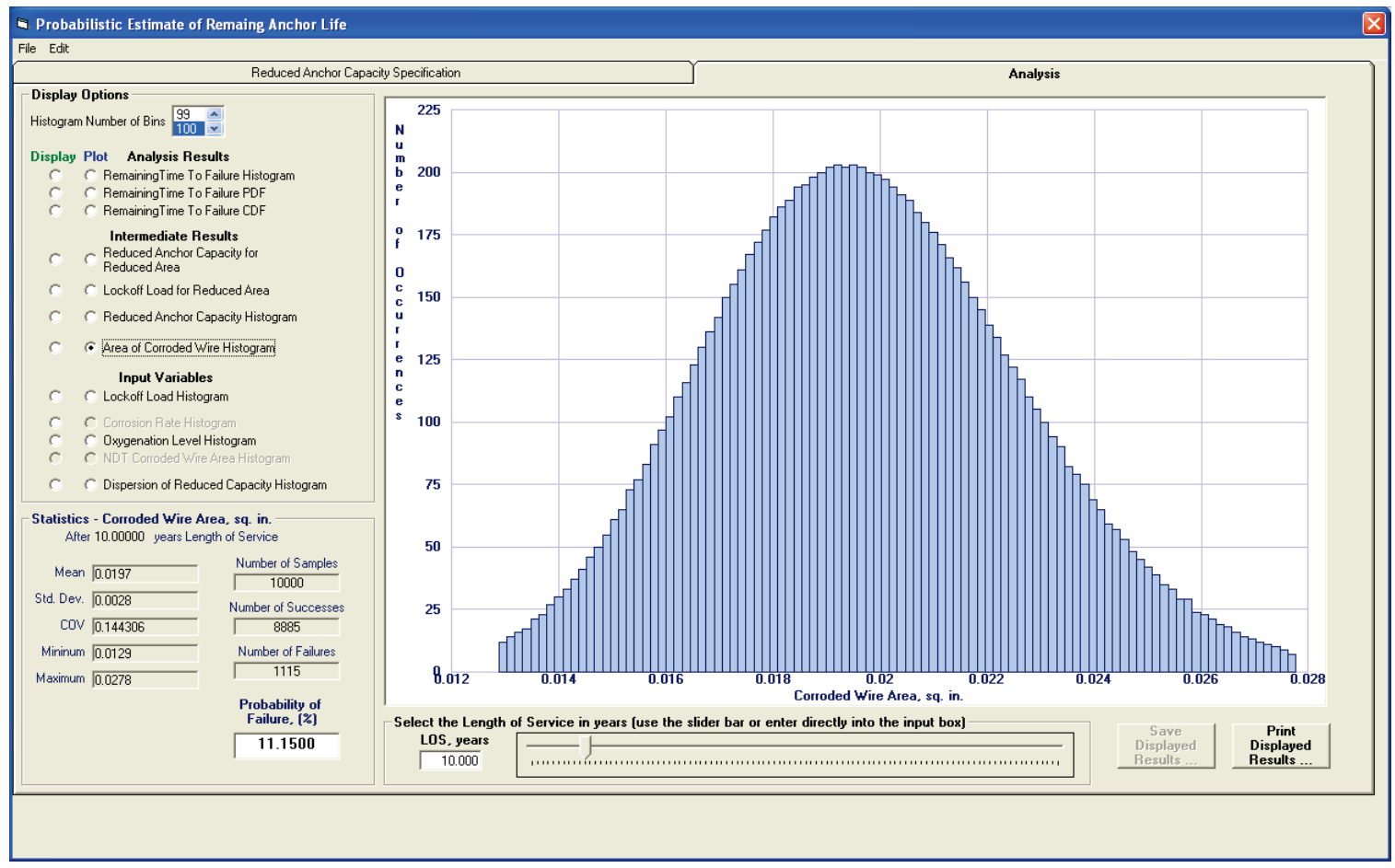




\section{Lock-off Load Histogram}

Select the Lock-off Load Histogram option button in the Plot column to produce a histogram of the lock-off load values. This provides the user a visual confirmation of an appropriate distribution of values. A sample lock-off load histogram is shown in Figure 3-92. The actual histogram data points and the lock-off load data points may be viewed in tabular list form by selecting the Lock-off Load Histogram button in the Display column. The tabular list format is the same as described in section 3.5.3.1 and shown in Figure 3-83 previously.

Figure 3-92. Lock-off Load Histogram Plot.

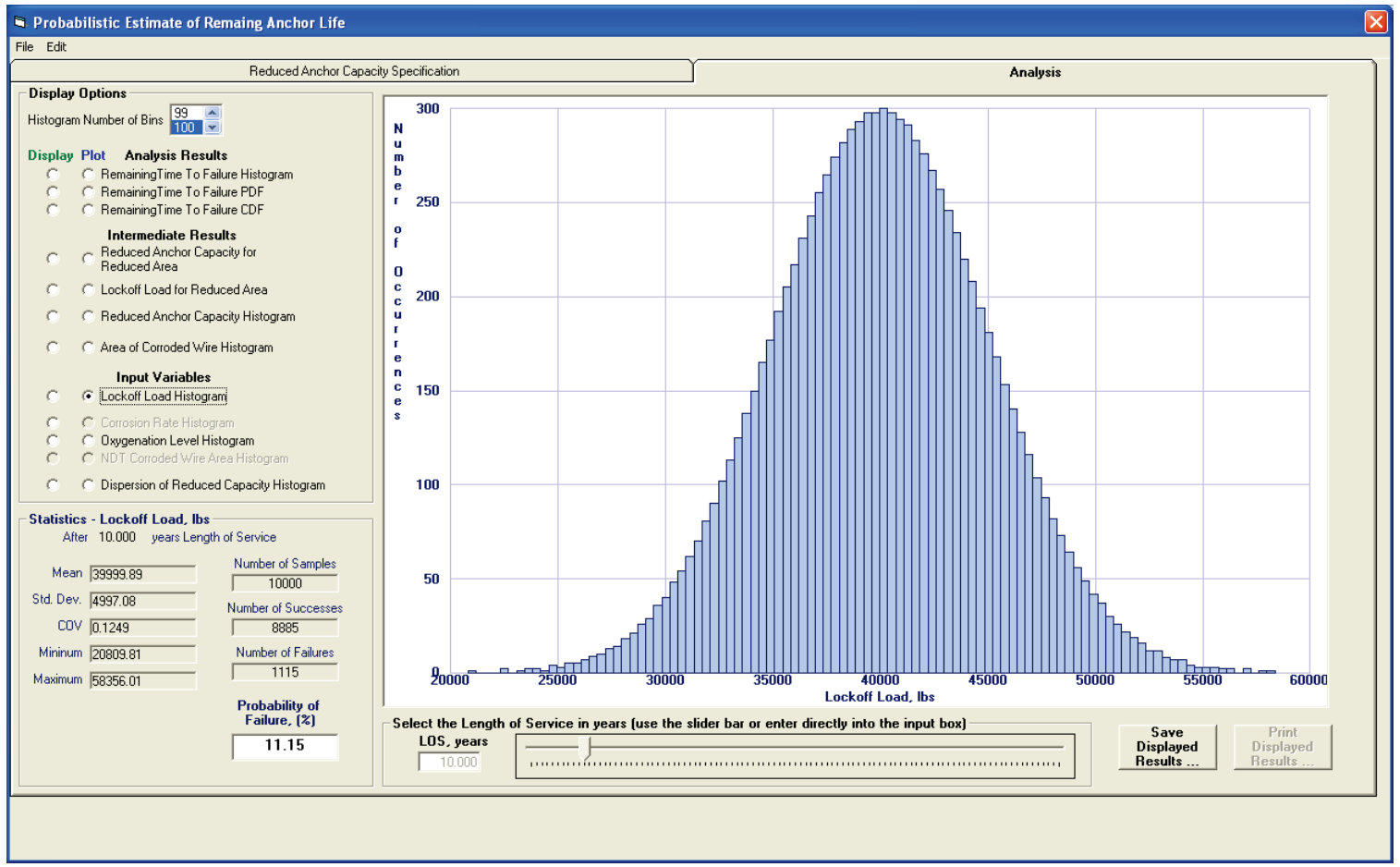

\section{Corrosion Rate Histogram}

Select the Corrosion Rate Histogram option button in the Plot column to produce a histogram of the user-specified corrosion rate values. This provides the user a visual confirmation of an appropriate distribution of values. A sample corrosion rate histogram is shown in Figure 3-93. The individual histogram data points and the corrosion rate data points may be viewed in tabular list form by selecting the Corrosion Rate Histogram button in the Display column. The tabular list format is the same as described in section 3.5.3.1 and shown in Figure 3-83 previously. 
Figure 3-93. Corrosion Rate Histogram.

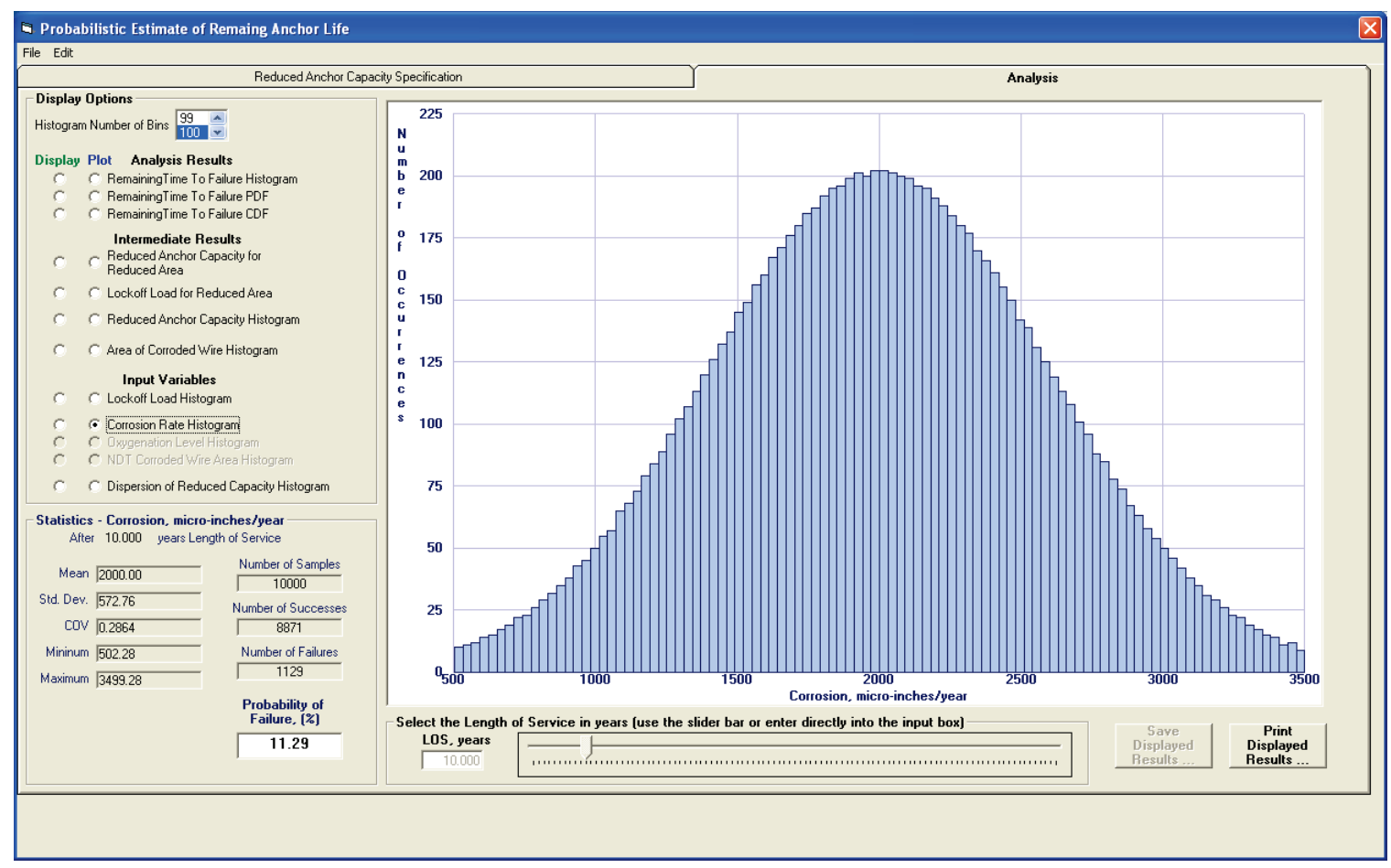

\section{Oxygenation Level Histogram}

Select the Oxygenation Level Histogram option button in the Plot column to produce a histogram of the user-specified oxygenation level values. This provides the user a visual confirmation of an appropriate distribution of values. A sample oxygenation level histogram is shown in Figure 3-94. The individual histogram data points and the oxygenation level data points may be viewed in tabular list form by selecting the Oxygenation Level Histogram button in the Display column. The tabular list format is the same as described earlier in this section and shown in Figure 3-83.

\section{NDT Corroded Wire Area Histogram}

Select the NDT Corroded Wire Area Histogram option button in the Plot column to produce a histogram of the NDT-determined wire area values. This provides the user a visual confirmation of an appropriate distribution of values. A sample NDT corroded wire area histogram is shown in Figure 3-95. The individual histogram data points and the NDT corroded wire area data points may be viewed in tabular list form by selecting the NDT Corroded Wire Area Histogram button in the Display column. The tabular list format is the same as described earlier in this section and shown in Figure 3-83. 
Figure 3-94. Oxygenation Level Histogram.

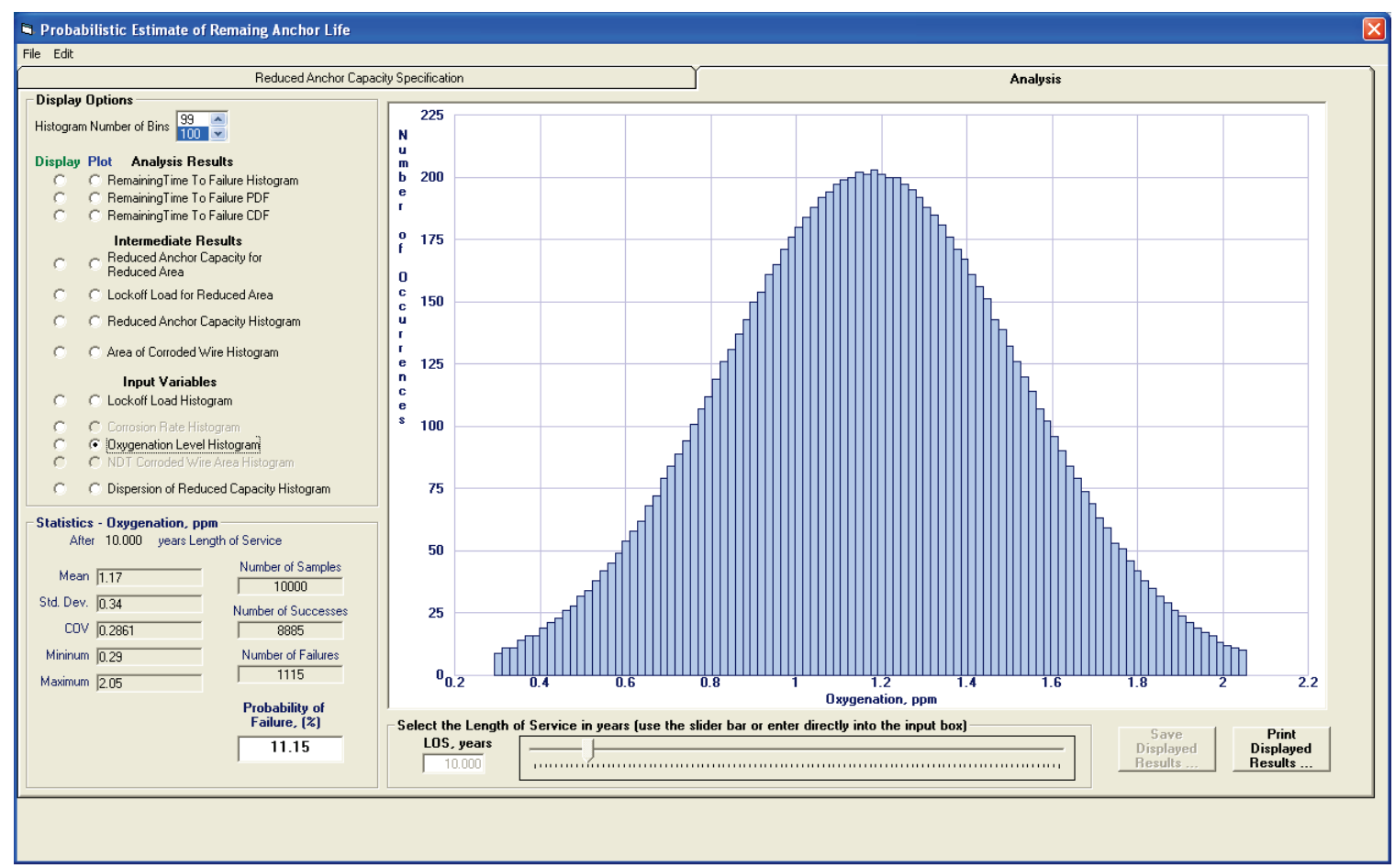

Figure 3-95. NDT Corroded Wire Area Histogram.

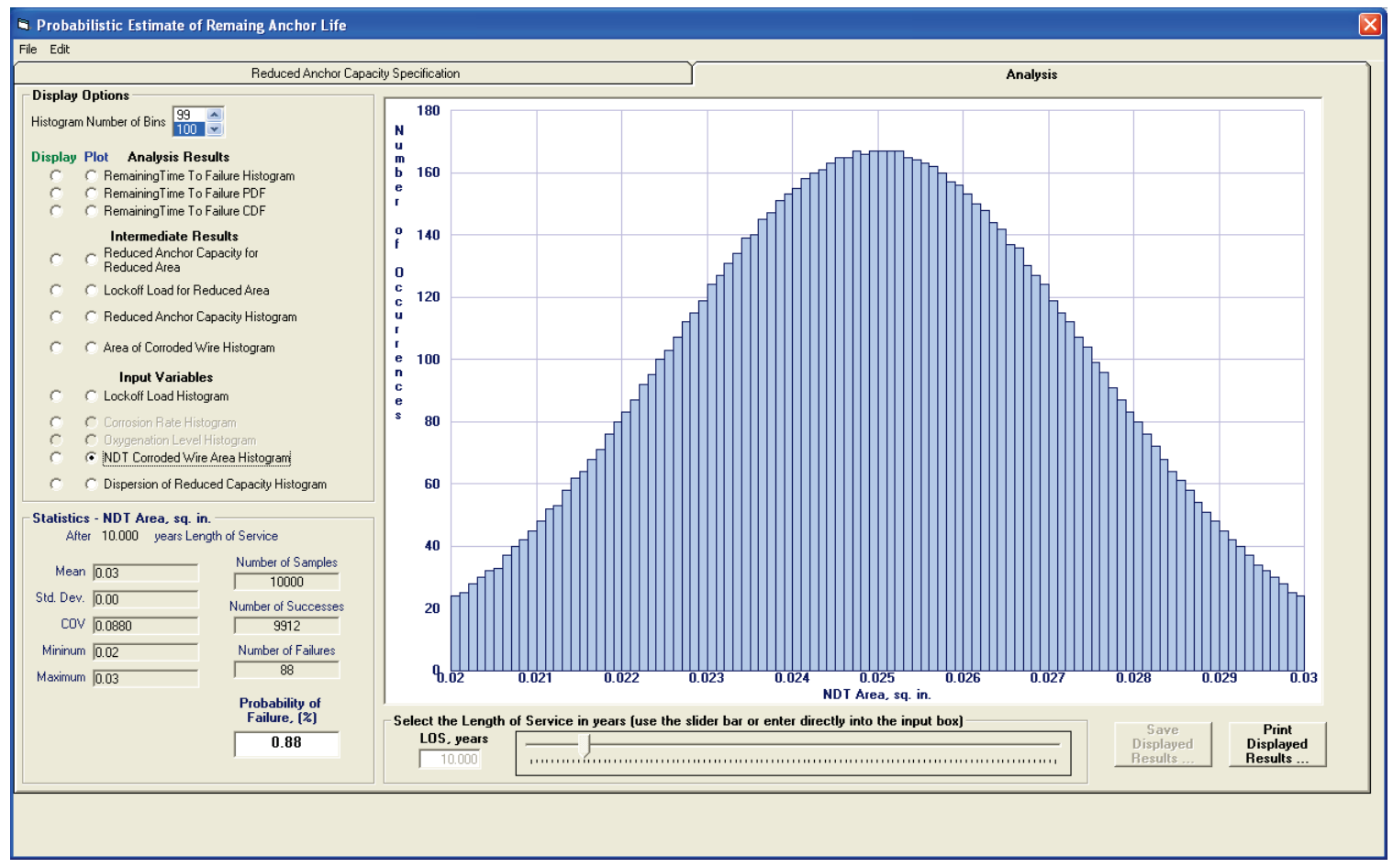




\section{Dispersion of Reduced Capacity Histogram}

Select the Dispersion of Reduced Capacity Histogram option button in the Plot column to produce a histogram of the reduced capacity dispersion values. This variable was discussed in section 3.5.2.5. This provides the user a visual confirmation of an appropriate distribution of values. A sample Dispersion of Reduced Capacity histogram is shown in Figure 3-96. Note the text in the Display Area: Std. Dev. $=4557$. This indicates the use of the standard deviation value for the data presented in Figure 3-78 as the scaling factor applied to the Dakota generated dispersion variable. The Dakotagenerated dispersion variable distribution is shown in Figure 3-81. The individual histogram data points and the reduced capacity dispersion data points may be viewed in tabular list form by selecting the Dispersion of Reduced Capacity Histogram button in the Display column. The tabular list format is the same as described in section 3.5.3.1 and shown in Figure 3-83 previously.

Figure 3-96. Dispersion of Reduced Capacity Histogram.

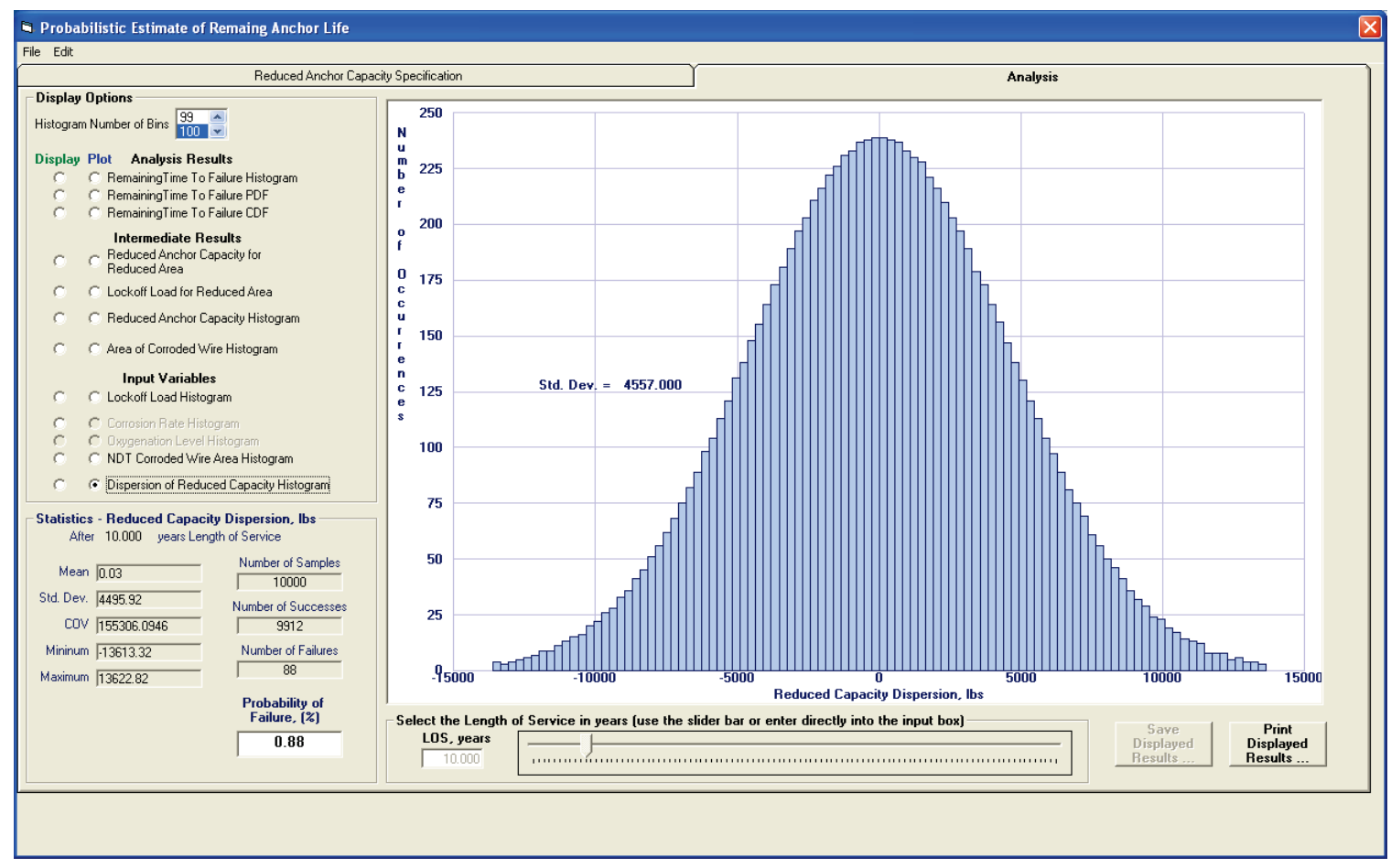

\subsubsection{Statistics frame}

The Statistics frame is located in the lower left corner of the Analysis tab. The statistical information in this frame is updated each time a change is made in the Display Area. The statistics reported are the mean (Mean), 
standard deviation (Std. Dev.), COV, minimum (Minimum) and maximum (Maximum) values. Also reported are the number of samples (Number of Samples) in the dataset, the number of samples with the reduced capacity greater than or equal to the lock-off load (Number of Successes), and the number of samples with the reduced capacity less than the lock-off load (Number of Failures). The Probability of Failure is the ratio of the Number of Failures and the Number of Samples and is reported as a percentage in the lower right corner of the frame. The LOS in years of the cable is listed at the top of the frame immediately below the frame title.

Figure 3-97 shows the Statistics frame for a TTF histogram for cable with 10 years LOS. For this example, the POF is $11.15 \%$ as 1,115 samples in the sample set of 10,000 samples will have failed after this length of time. For the samples that remain, the mean value of the remaining TTF is 9.18 years.

Figure 3-97. Statistics-Remaining Time to Failure After 10 years Length of Service.

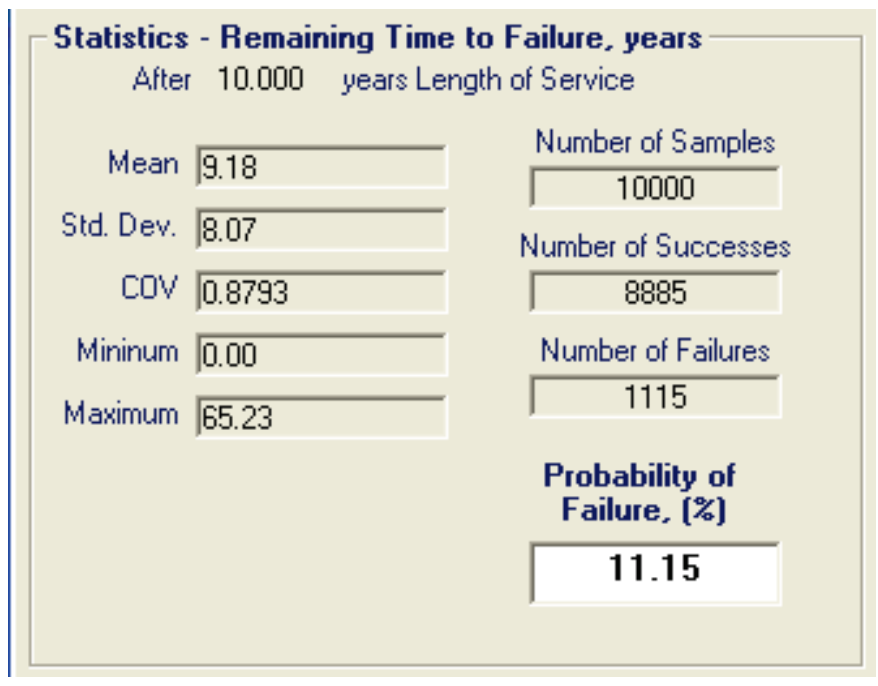

\subsubsection{Length of Service frame}

The Length of Service frame is located below the Display Area as shown in Figure 3-98. This frame provides two methods of selecting the LOS time in years. One method is to enter the value into the input box labeled LOS, years. If a partial year value is needed, it must be entered here. That is, if the LOS is 10 years and 6 months, the user should enter 10.5 into the input box. The second method is to use the mouse to move the slider left or right. As the slider is moved, the value in the LOS, years input box will be updated to indicate the current value. Only whole numbers are available to be selected with the slider. 
The LOS frame controls are enabled only for the options in the Analysis Results and Intermediate Results categories. Those values vary according to the LOS. The LOS frame controls are disabled for the options in the Input Variables category as the inputs remain constant regardless of the LOS.

Figure 3-98. LOS frame showing a selection of 10 years.

Select the Length of Service in years [use the slider bar or enter directly into the input box] LOS, years

10.000

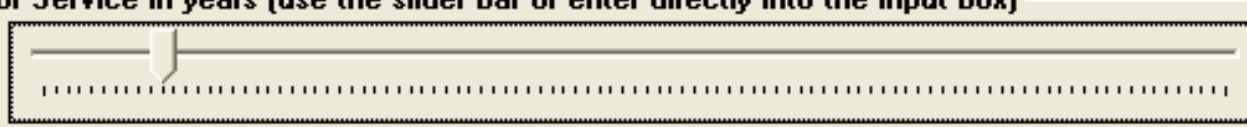

\subsubsection{Save Displayed Results and Print Displayed Results buttons}

Below the Display Area on the right side of the tab are the Save Displayed Results and Print Displayed Results buttons. These buttons provide the user the means to save the results currently in the Display Area.

When the Display Area contains a graphical display, the Print Displayed Results button is enabled, and the Save Displayed Results button is disabled as shown in Figure 3-99. Clicking the Print Displayed Results button will produce a Print dialog box as shown in Figure 3-100. Select the desired print device to use, adjust settings as desired, and click OK. The contents of the Display Area will be sent to the chosen print device.

When the Display Area contains a tabular list, the Save Displayed Results button is enabled and the Print Displayed Results button is disabled as shown in Figure 3-101. Clicking the Save Displayed Results button will produce a Save dialog box as shown in Figure 3-102. Select the type of file in the drop-down list box labeled Save as type:. Enter the name of the file in the File name: box or select it from the list of files. Click the Save button. The contents of the Display Area will be saved to the selected file. In this case, the contents of the Display Area include the entire list box, not only those that are currently in view.

The format of the data in the file depends on the type of file that was chosen. If the file type chosen is ASCII Data (* .dat), the data will appear as shown in the Display Area. If the type chosen is Comma Separated Variables (*.csv), the data will be saved as a .csv file. This format provides for easier loading into a spreadsheet file. 
Figure 3-99. Enabled Print Displayed Results button.

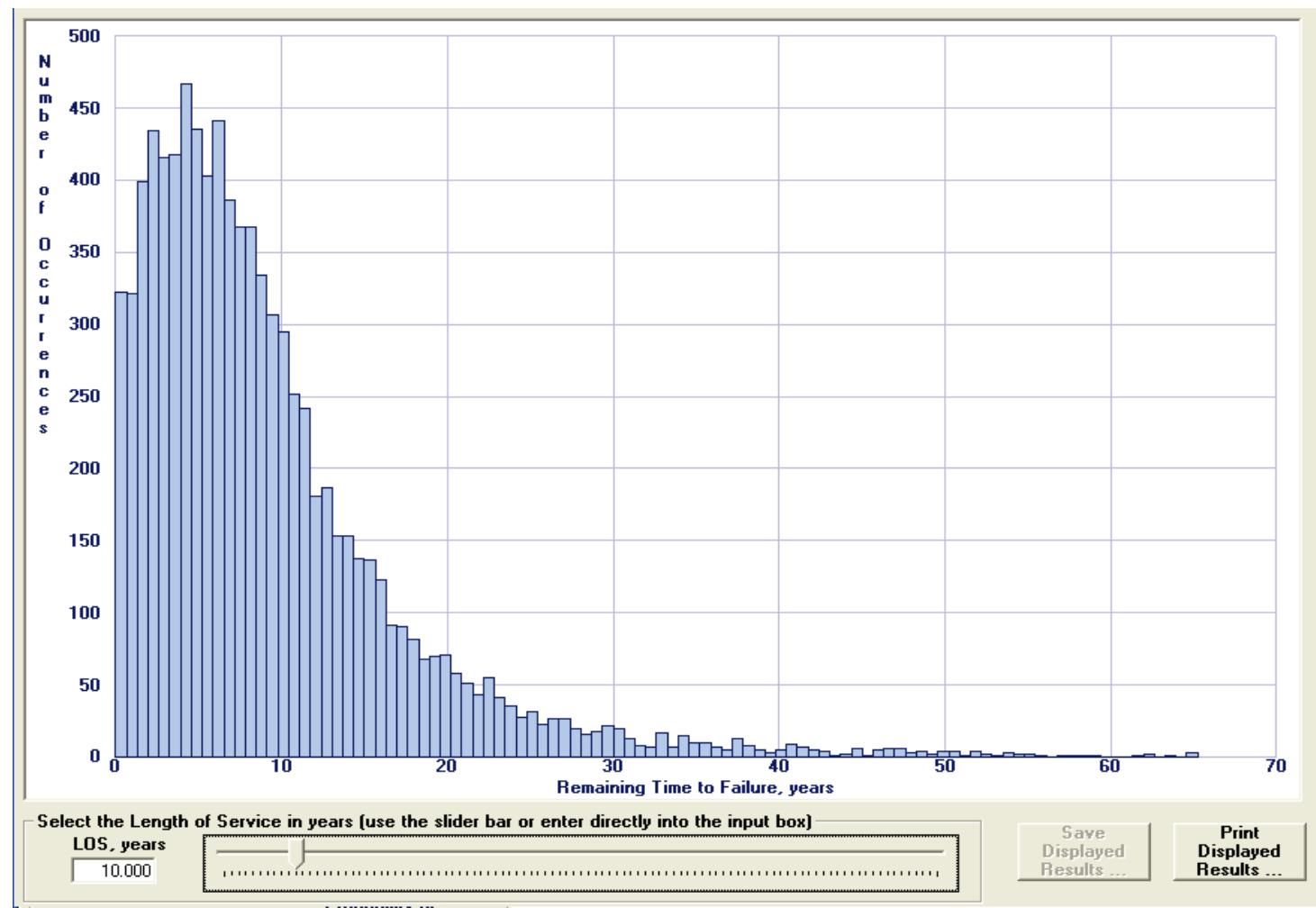

Figure 3-100. Print dialog box.

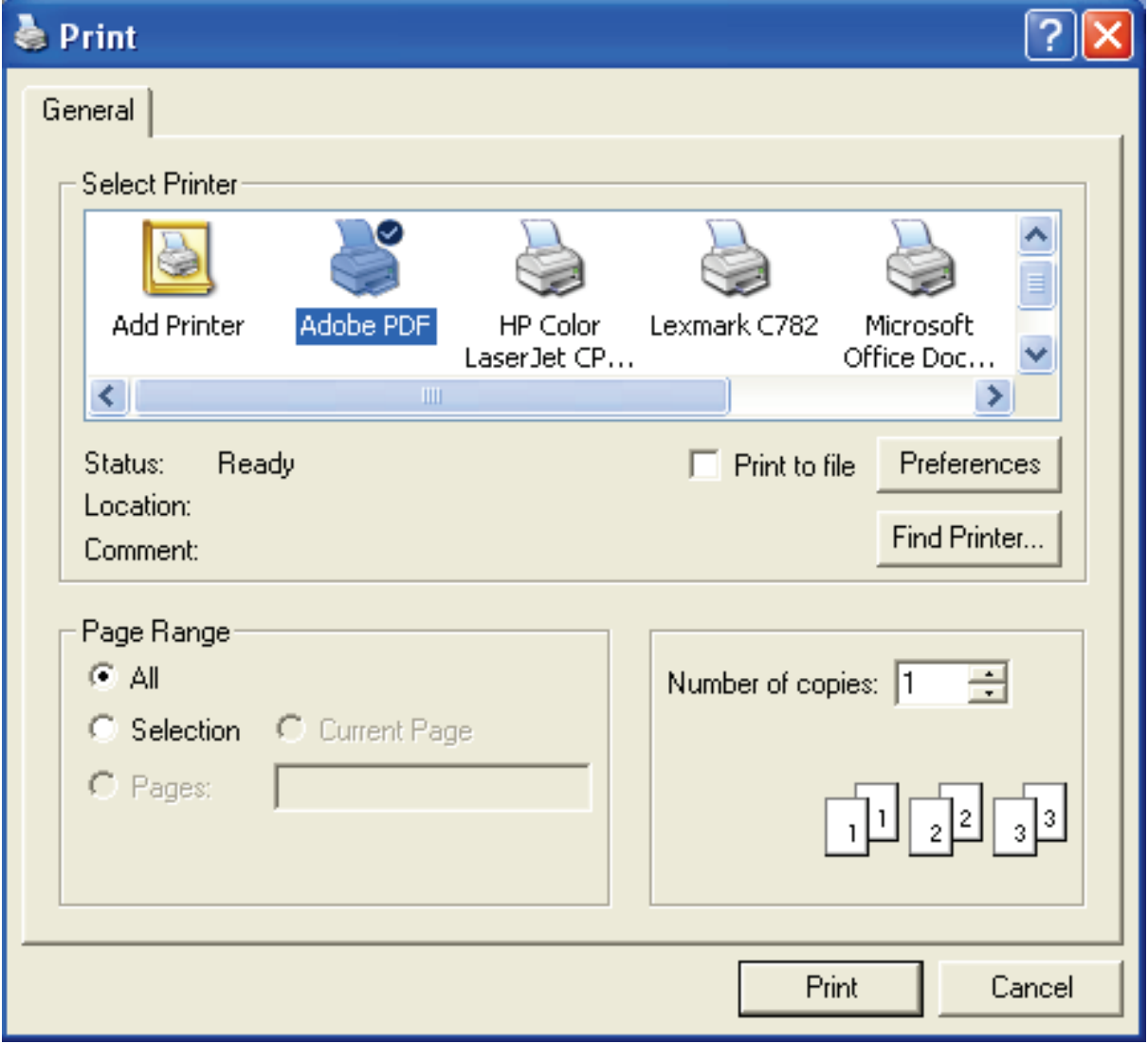


Figure 3-101. Enabled Save Displayed Results button.

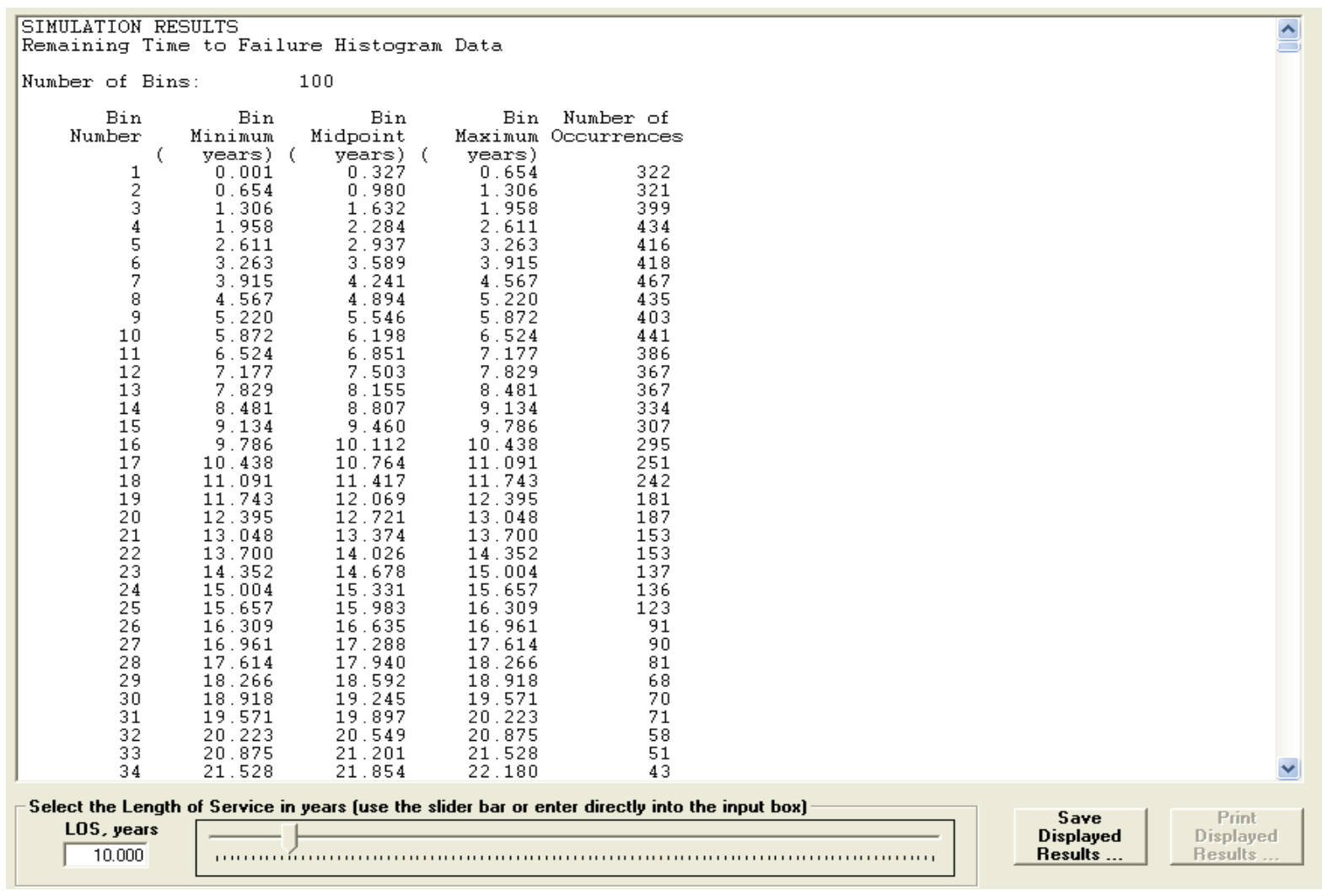

Figure 3-102. Save dialog box.

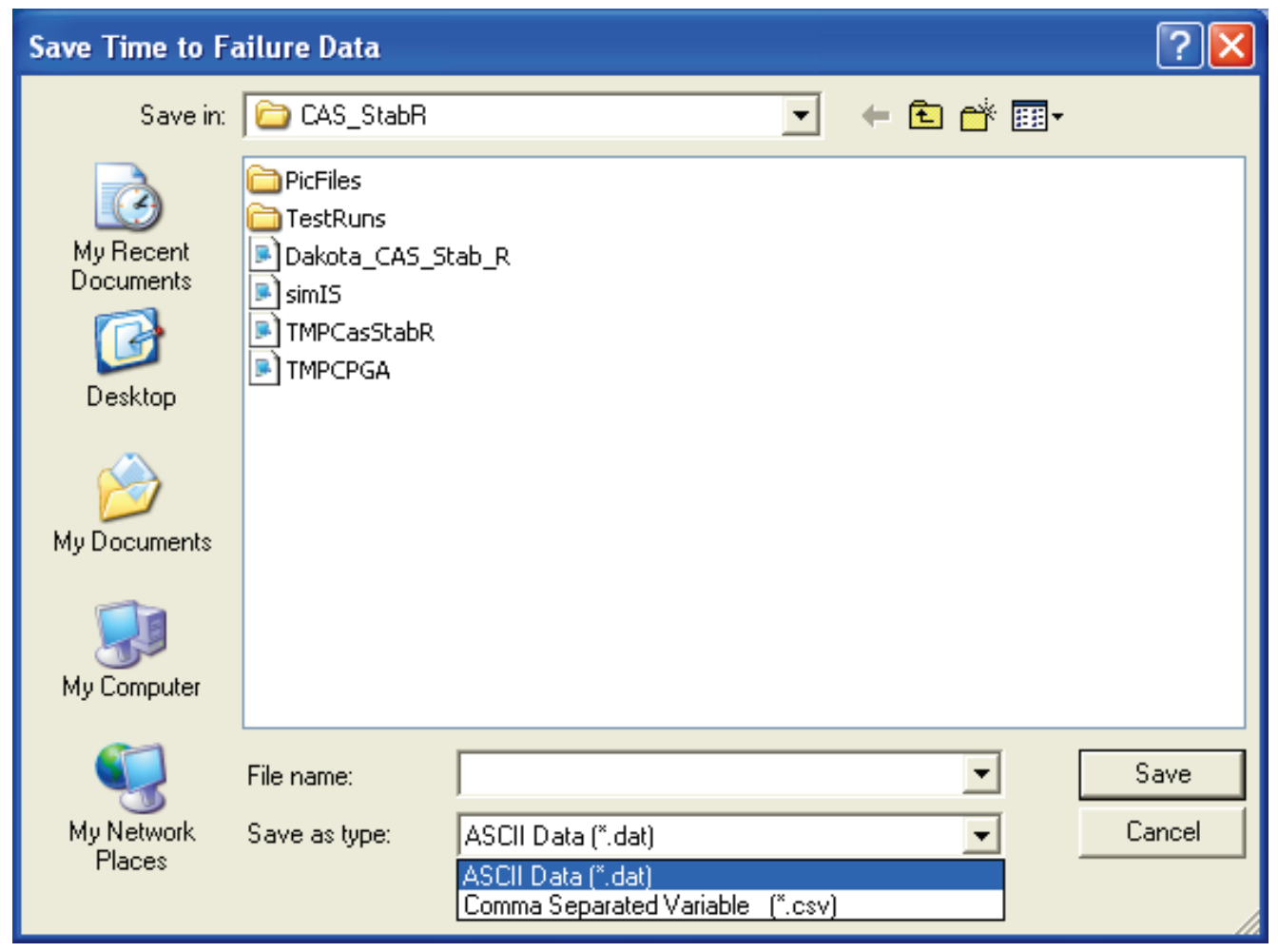




\section{Example Problems}

\subsection{Introduction}

This chapter discusses the probabilistic analysis of two sample problems performed with the Visual Modeler for CAS_Stab-R software. The first problem is an RAL analysis of a stranded cable with a known lock-off cable loading and an estimated oxygenation level of the environment surrounding the corroding cable. The second problem is an analysis of the structural stability against sliding of a navigation lock wall possessing corroded anchorage.

A third problem presented in this report is an analysis of the stability of a dam, as defined in ETL 1110-2-256 (HQUSACE 1981), against sliding. This is a deterministic analysis that serves to verify the ETL 1110-2-256 (HQUSACE 1981) multiple wedge solution formulation that was implemented in this CAS_Stab-R software. Because it serves as a validation analysis and possesses no anchorage, it is summarized in Appendix B rather than in this chapter.

\subsection{Example 1 - RAL probabilistic analysis}

This analysis will demonstrate the use of CAS_Stab-R to determine a probabilistic assessment of TTF for a seven-strand anchor cable due to corrosion. The analysis is performed for a cable with a tensile lock-off force with a mean value of 40 kips and a mean level of oxygen in the anchor environment of $1.175 \mathrm{ppm}$.

The lock-off force and oxygenation level distributions are assigned on the Reduced Capacity Variable Input tab of the LoA window. Figure 4-1 shows the distribution for the lock-off force. The distribution type chosen is a bounded normal distribution. The mean value is assigned to be $40 \mathrm{kips}$. The standard deviation is assigned $5 \mathrm{kips}$, which yields a COV of 0.125. The minimum (lower bound of possible values) is assigned 20 kips, which is equal to the mean minus 4 times the standard deviation. The maximum (upper bound of possible values) is assigned $60 \mathrm{kips}$, which is equal to the mean plus 4 times the standard deviation. 
Figure 4-1. Initial Lock-off Force distribution for Example 1.

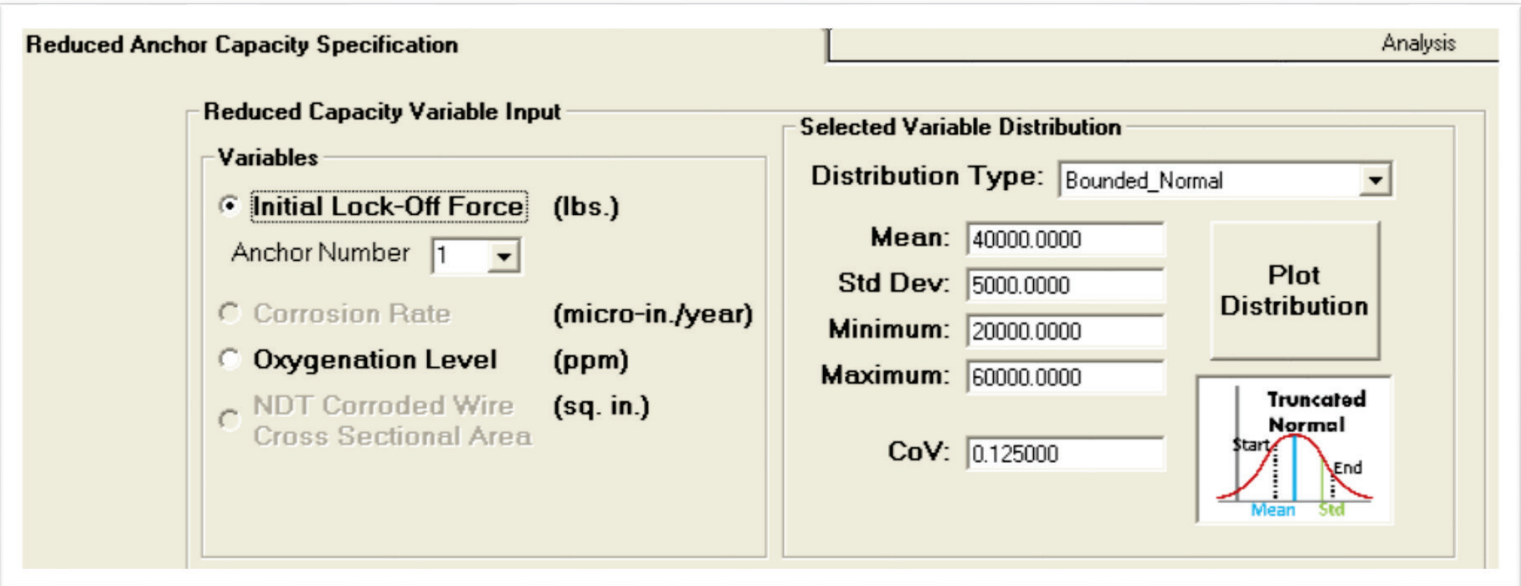

Since the oxygenation level of the anchor's surroundings is the measure to determine the amount of corrosion occurring over time, the Oxygenation Level variable must be activated for use. This selection is also made on the Reduced Capacity Variable Input tab in the Select the Corrosion Method frame. Figure 4-2 shows this frame with the Oxygenation Level option chosen. The distribution for the Oxygenation Level is assigned a bounded normal distribution type with a mean value of $1.175 \mathrm{ppm}$ and a standard deviation of $0.3520 \mathrm{ppm}$, which yields a COV of 0.3. (Figure 4-3) The minimum (lower bound of possible values) is assigned $0.293 \mathrm{ppm}$. The maximum (upper bound of possible values) is assigned 2.055 ppm. The upper and lower bound values are equal to the mean Oxygenation Level plus 2.5 times the standard deviation and the mean minus 2.5 times the standard deviation, respectively.

Figure 4-2. Oxygenation Level selected as the corrosion criteria.

Select the Corrosion Method

C Corrosion Rate

- Oxygenation Level

S NDT Corroded Wire Cross Sectional Area 
Figure 4-3. Oxygenation Level distribution assignment.

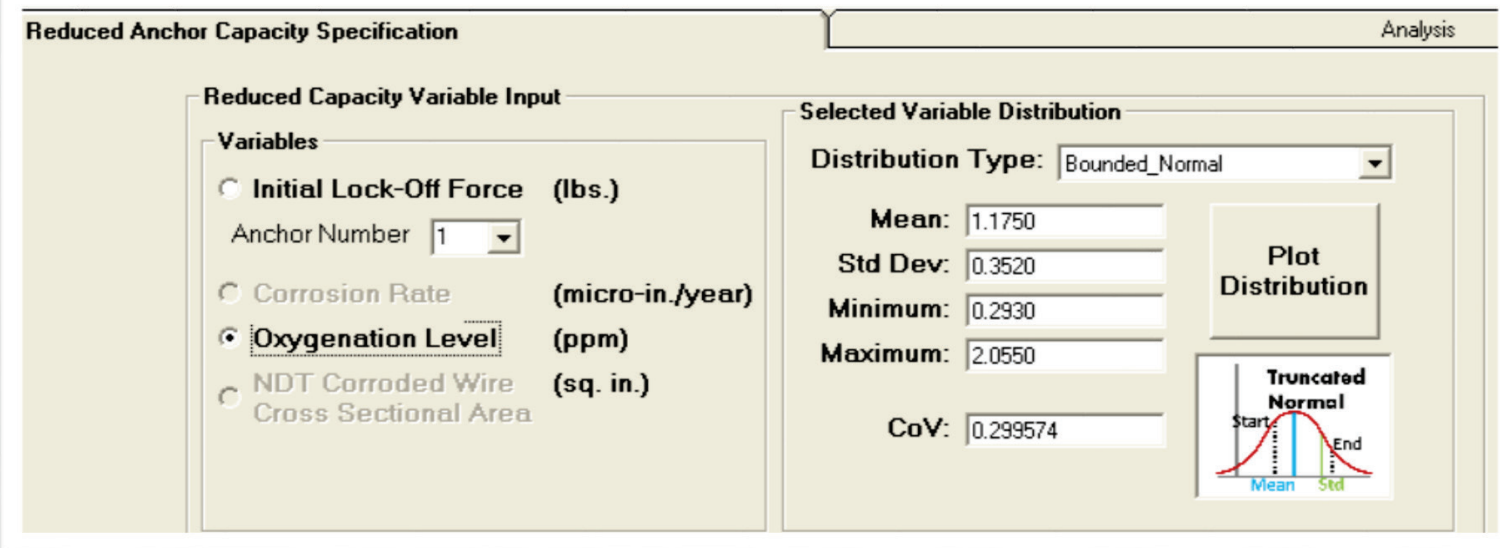

With the variable distributions assigned, a Dakota dataset of sample points must be generated. Do this by clicking the Generate the Dakota Data Set button found on the Reduced Anchor Capacity Specification tab. The number of samples in the dataset is chosen as 10,000 in the Number of Simulations input window that appears in response to the button click. This input window is shown in Figure 4-4. Following the dataset creation, the Estimate the Remaining Anchor Life button is enabled as shown in Figure 4-5.

Figure 4-4. Number of Simulations input window.

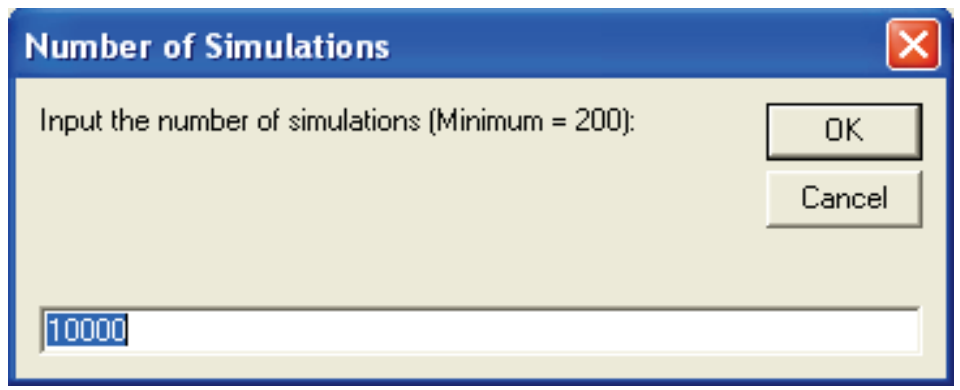

Figure 4-5. Estimate the Remaining Anchor Life button enabled.

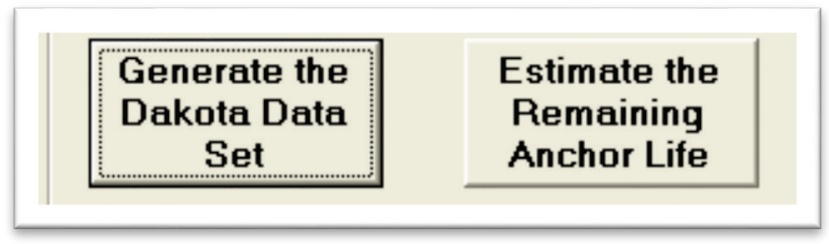


Prior to obtaining the estimated RAL, the Simplified Average Corrosion Calculation curve to utilize in determining the reduced capacity of the corroded cable must be selected. For this example, the Corroded Wire Area curve is chosen as shown in Figure 4-6.

Figure 4-6. Simplified Average Corrosion Calculation curve selection.

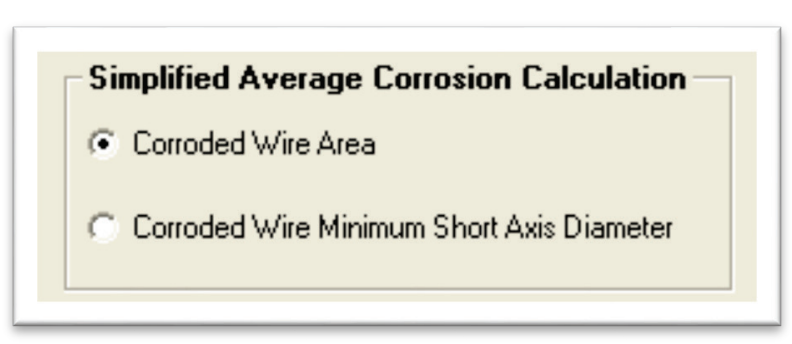

Clicking the Estimate the Remaining Anchor Life button switches the window to the Analysis tab. When the Analysis tab loads, the histogram plot of the Remaining Time to Failure option is selected, and the Analysis tab shown in Figure 4-7 is seen.

Figure 4-7. Remaining Time to Failure Histogram for 0 years LOS for Example 1.

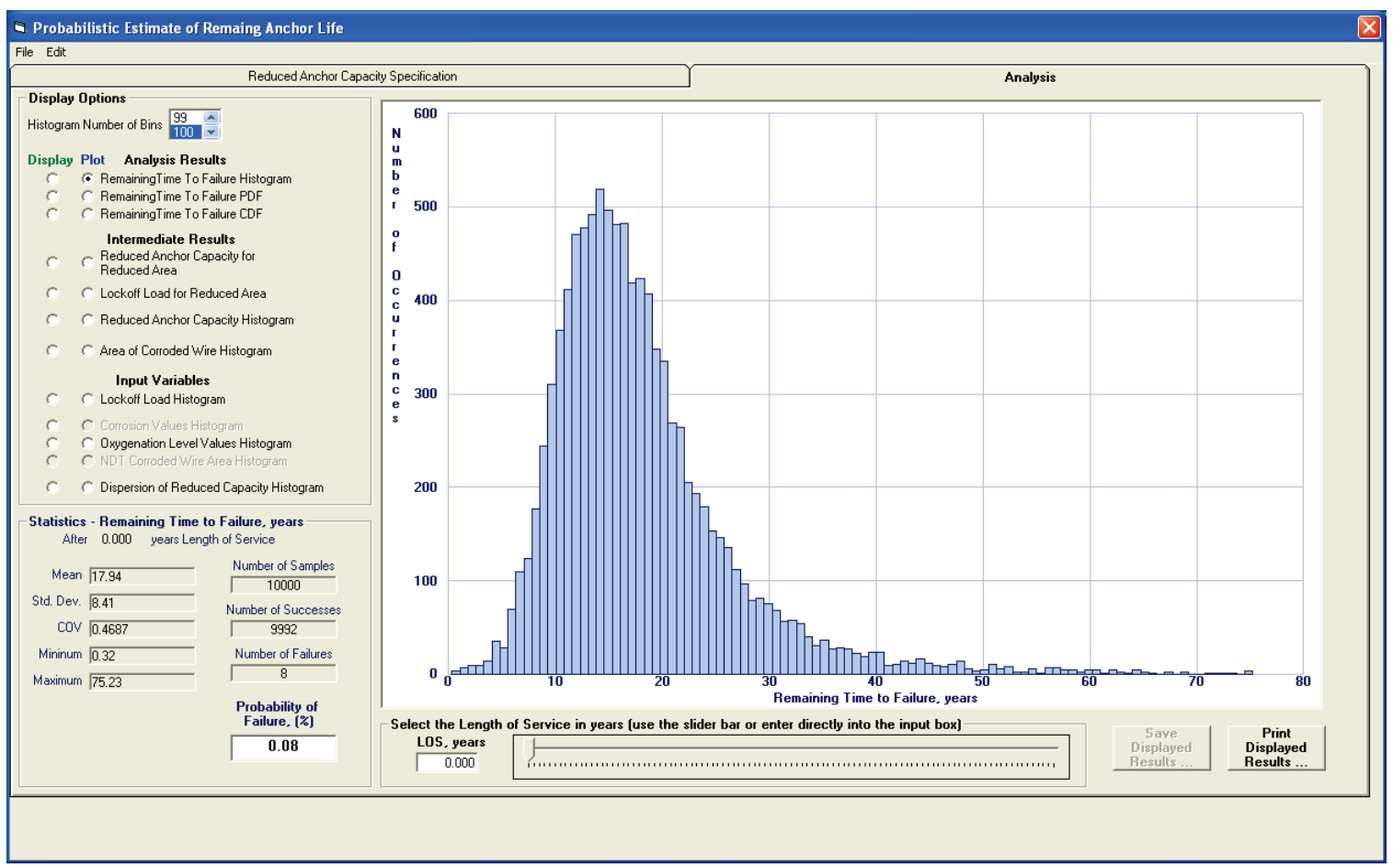

From this figure, it is seen that at installation designated by o years LOS, the mean estimate of TTF of the anchor is calculated to be equal to 17.94 years. This mean value is seen in the Statistics frame. Also, the Statistics 
frame shows that the POF of the anchor is only 0.08\%. The POF indicates that only 8 samples out of 10,000 samples in the simulation resulted in the lock-off force applied to the cable exceeding the reduced capacity of the cable.

Changing the LOS value will yield new results as shown in Figure 4-8. In this figure, the LOS is set to 10 years. The statistics shown (the mean [Mean], standard deviation [Std. Dev.], COV, minimum [Minimum] and maximum [Maximum] values) apply only to the samples for which the reduced capacity exceeds the lock-off force. That is, the statistics are computed for the 8,885 samples shown as the Number of Successes in the Statistics box. For this LOS, a mean TTF is observed as 9.18 (additional) years for the successful samples with respect to the "current" time of 10 years. Also seen in the Statistics box is the POF of $11.15 \%$.

Figure 4-8. Remaining Time to Failure Histogram for 10 years LOS for Example 1.

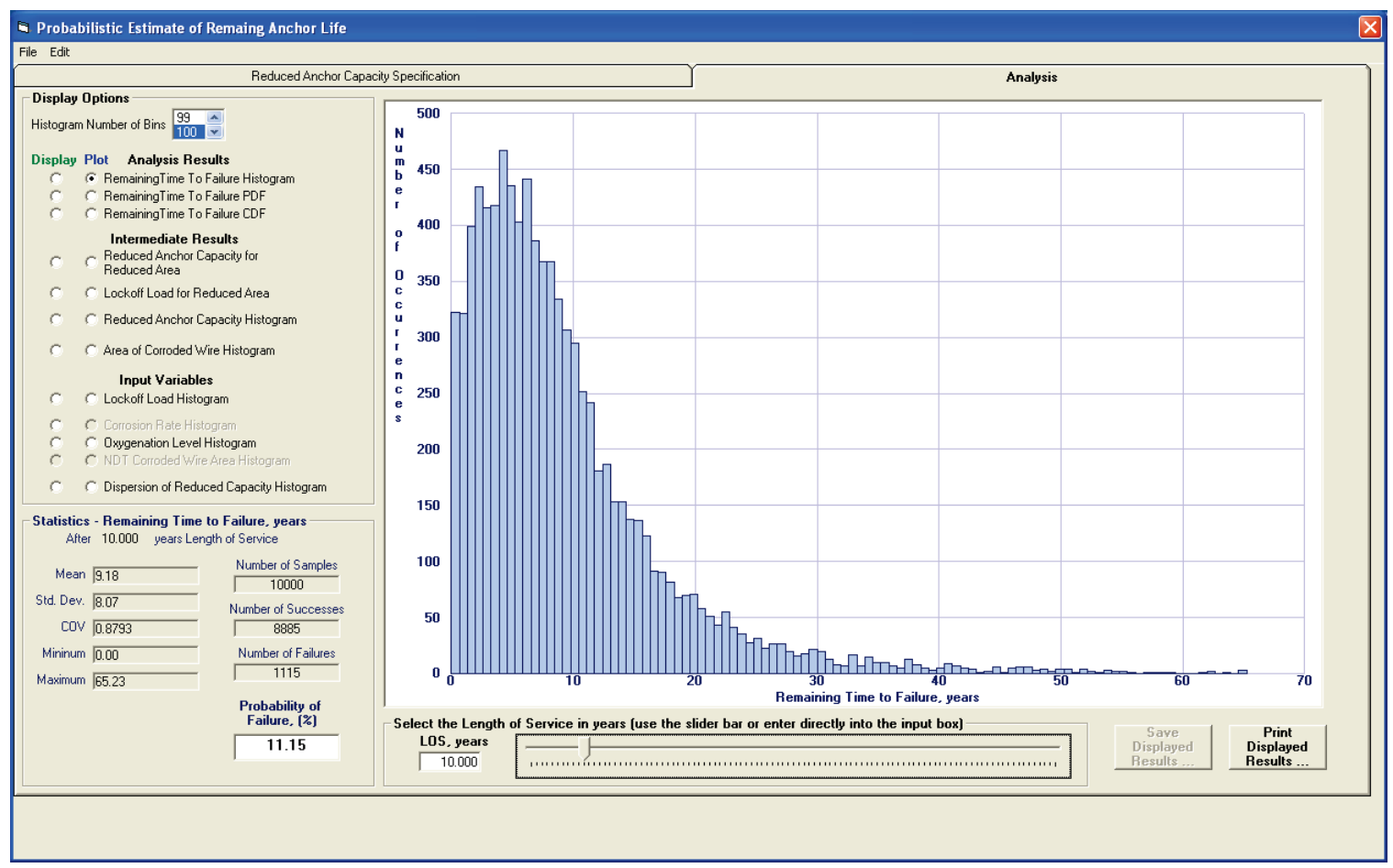

To determine the time at which a 95\% statistical certainty that all samples will have failed exists, the LOS can be increased until the POF is observed to be $95 \%$. Performing this exercise with this example yielded an LOS of 33.3 years. This result is shown in Figure 4-9.

Additionally the time at which a 95\% statistical certainty that all samples will have failed can be determined by the use of the Remaining Time to 
Failure CDF plot with the LOS set to o years. This plot is shown in Figure 4-10. It is shown graphically that at 33 years, the CDF has reached a value of 0.95 .

Figure 4-9. Remaining Time to Failure Histogram for 33.3 years LOS for Example 1.

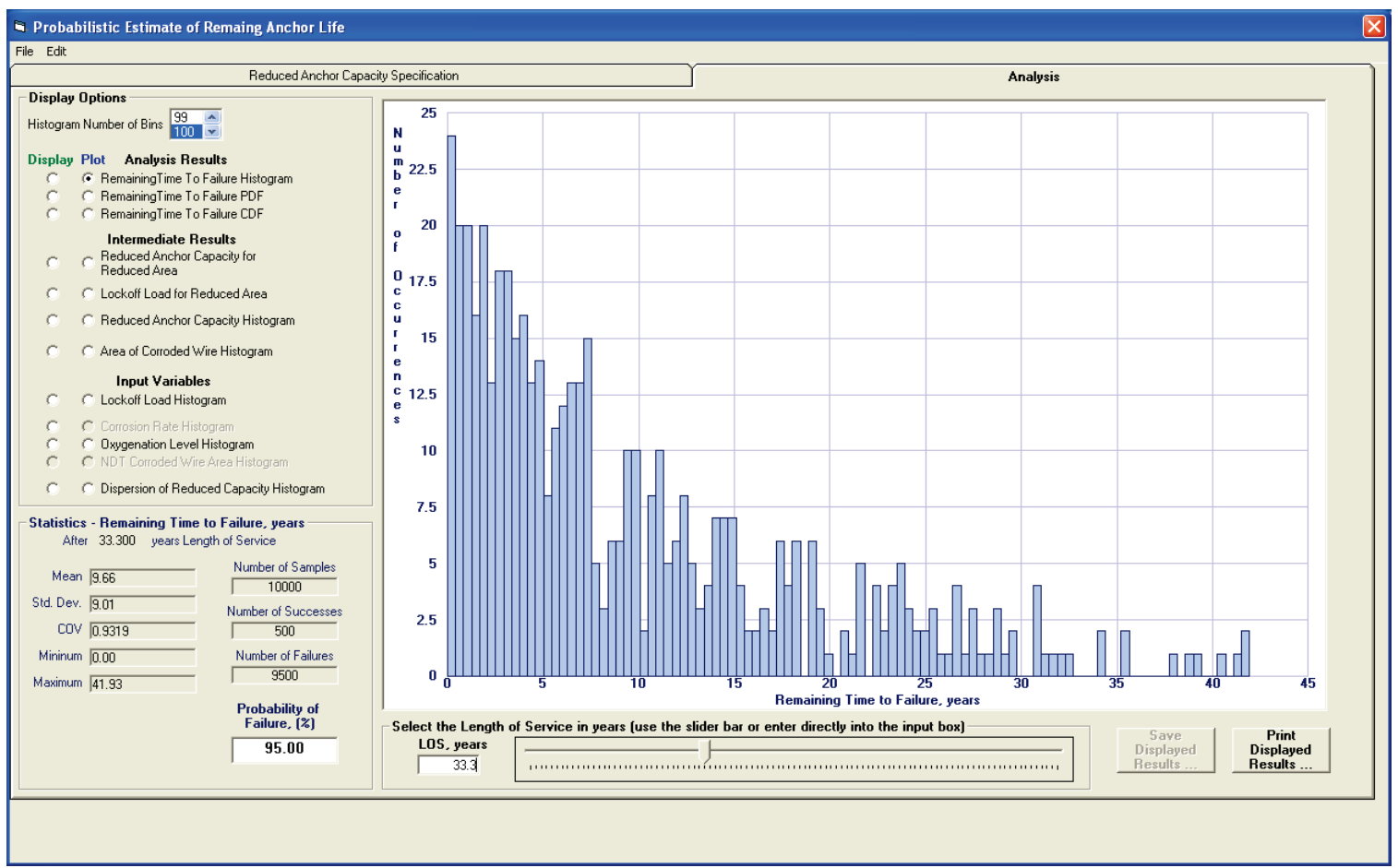

Figure 4-10. Example 1 Remaining Time to Failure CDF plot at LOS of 0 years.

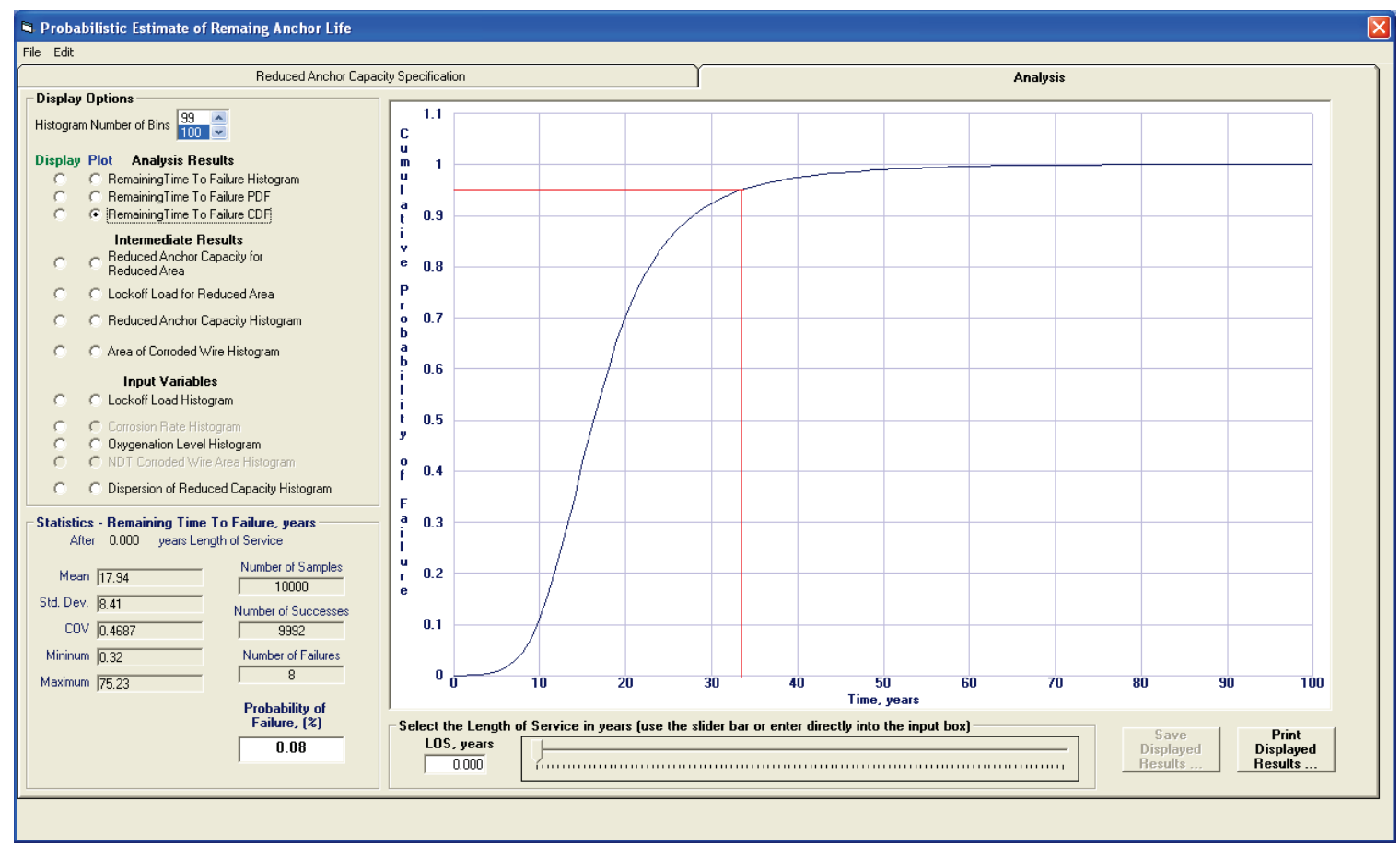




\subsubsection{Quality of dataset input variables - RAL example}

The input variable distribution histograms are viewed to verify an adequate number of samples were selected (i.e., 10,000) for a proper simulation. Figure 4-11 shows the resulting histogram for the lock-off force. The mean value of the lock-off force is computed to be 40 kips with a standard deviation of 4,997 lb. A minimum value of 20.89 kips and a maximum value of 58.36 kips were generated during the 10,000 simulations. This is in agreement with the distribution specified on the Reduced Anchor Capacity Specification tab.

Figure 4-12 shows the resulting histogram for the oxygenation level. The mean value of the oxygenation level is computed to be $1.17 \mathrm{ppm}$ with a standard deviation of $0.35 \mathrm{ppm}$. A minimum value of $0.29 \mathrm{ppm}$ and a maximum value of $2.05 \mathrm{ppm}$ were generated during the 10,000 simulations. This distribution is judged to be a good representation of the distribution specified on the Reduced Anchor Capacity Specification tab.

Figure 4-11. Example 1 Lock-off Load Histogram.

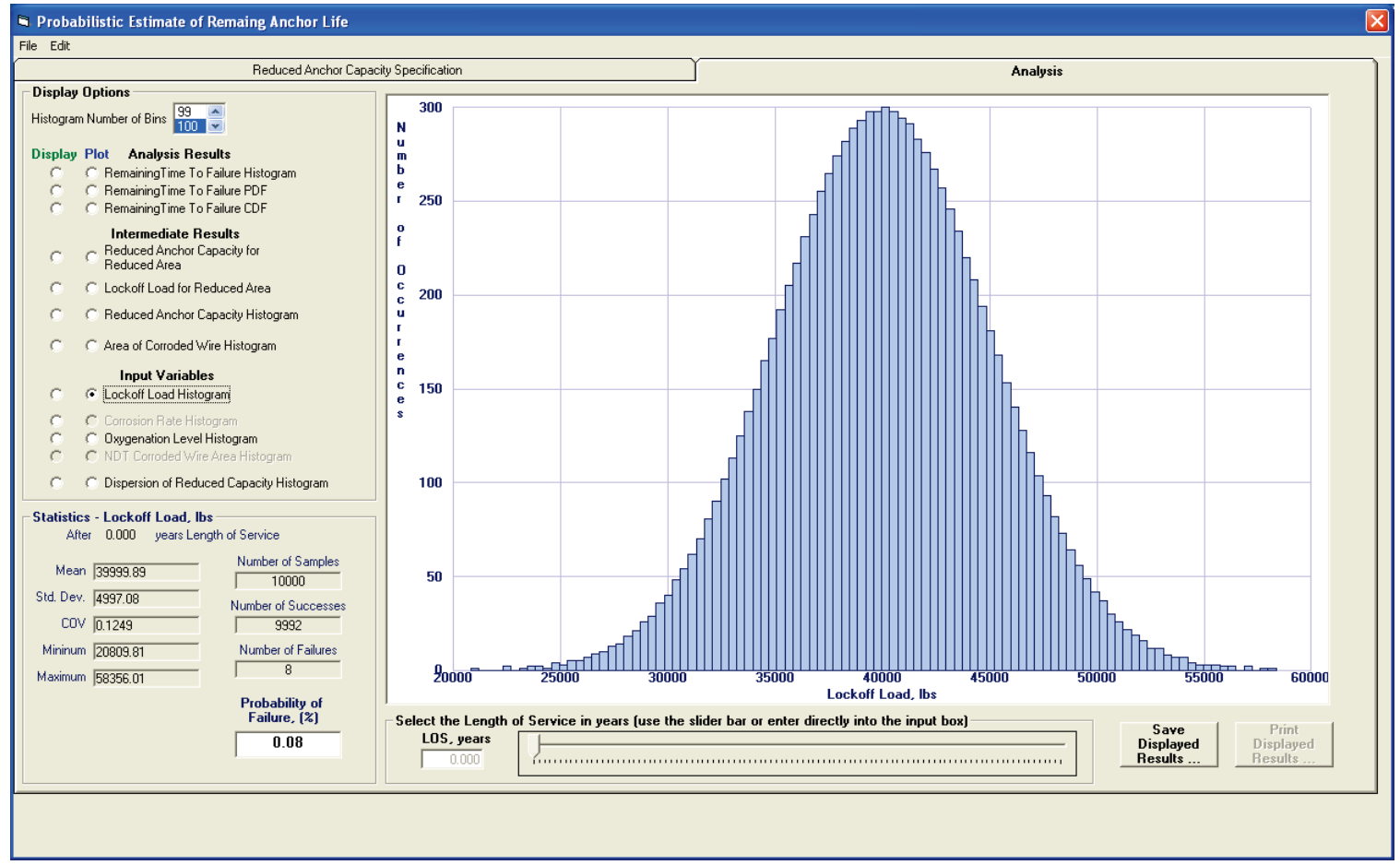


Figure 4-12. Example 1 Oxygenation Level Histogram.

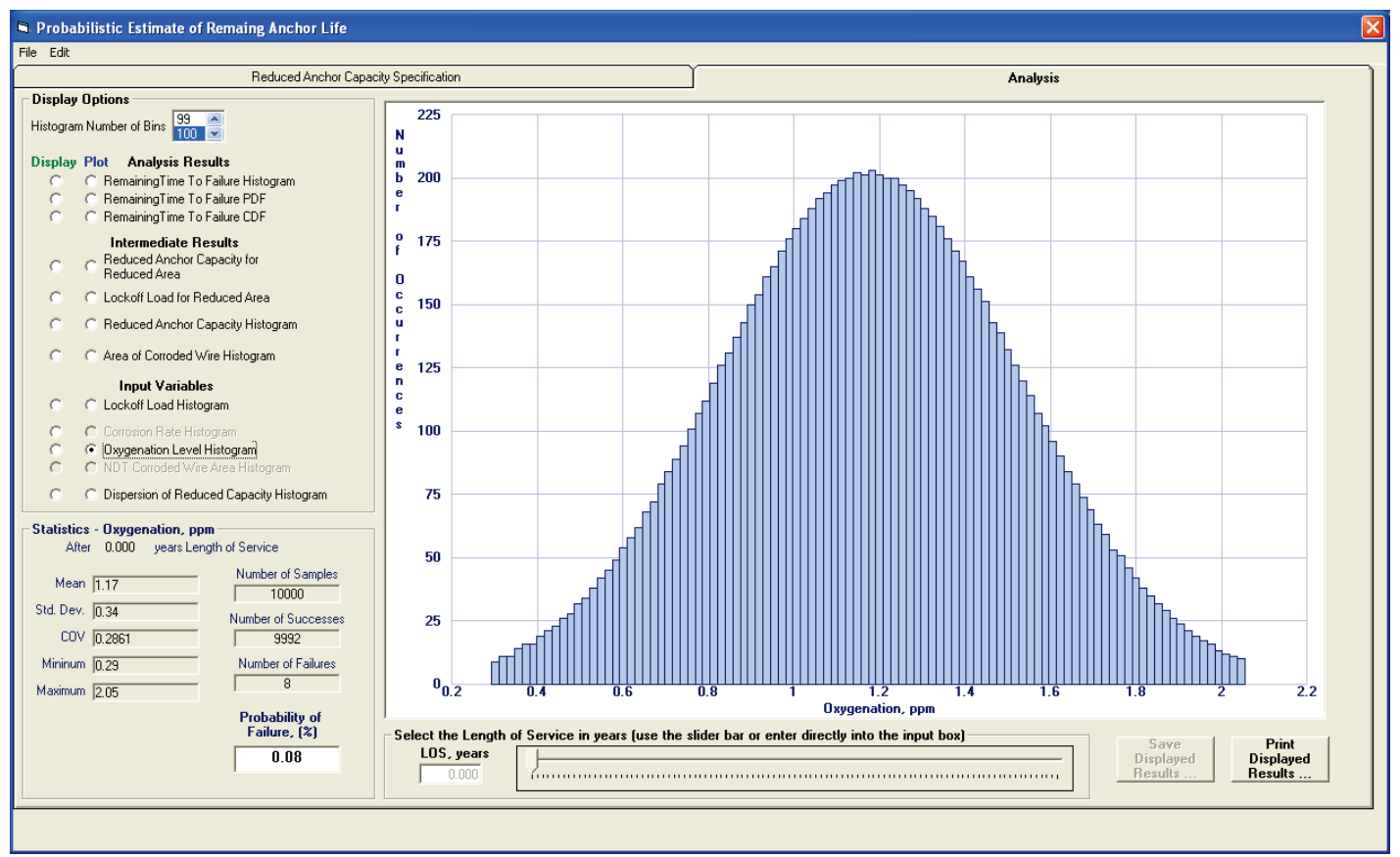

For each of these 10,000 simulations, the Ebeling et al. (2016) relationship between reduced cable capacity and corrosion-induced, reduced crosssectional area for a seven-wire strand cables is being used in CAS_Stab-R to calculate the (reduced) capacity of corroded cables. Figure 5.11 in Ebeling et al. (2016) shows the ERDC corrosion-bed-generated test data and the derived statistical relationships. This figure is also reprinted in this report and is labeled Figure 3-78. The derived statistical relationships of mean, mean plus one standard deviation, etc., of reduced cable capacity (in units of pounds force) are the labeled trendlines in this figure. The minimum wire area after corrosion takes place is plotted along the horizontal axis. The underlying distribution that characterizes the dispersion in the reduced cable capacity is a normal distribution. For the selected minimum wire area trendline equation, the samples have a standard error of 4,557 lb. The standard deviation corresponds to this standard error value. To account for statistical dispersion in the CAS_Stab-R simulation analysis using the Ebeling et al. (2016) Figure 5.11 trendlines, a simulation is needed. Each simulation starts out using a normalized normal distribution (i.e., PDF) with a mean of zero and a standard deviation of one. For each resulting corrosion induced, cross-sectional area, this normalized normal distribution simulation result is multiplied by 4,557 lb and added to the Ebeling et al. (2016) Figure 5.11 mean trendline value of cable capacity. This results in the simulated value of reduced cable capacity (in pounds). This process is then repeated for the next reduced corroded area simulation until all 10,000 simulations are completed. 
Figure 4-13 shows the resulting histogram for the reduced anchor capacity dispersion from the process described in the previous paragraph. The mean value of the generated dispersion is $0.04 \mathrm{lb}$ with a standard deviation of 4,496 lb. Ideally, the mean would be zero, and one standard deviation would be equal to one times $4,557 \mathrm{lb}$. This distribution is judged to be a good representation of the assignment made internally in the code of a mean value of $\mathrm{o}$, standard deviation of 1.0 to a normalized normal distribution. A mean value of 0.04 is quite close to the desired zero value, and a standard deviation value of $4,496 \mathrm{lb}$ is close to the desired 4,557 lb value. A minimum value of $-13,603 \mathrm{lb}$ and a maximum value of $13,665 \mathrm{lb}$ were generated during the 10,000 simulations. This normalized normal distribution had a specified minimum value of -3 standard deviations and a maximum of 3 standard deviations. A scaling factor of $4,557 \mathrm{lb}$ is applied to each of the 10,000 simulated normalized normal distribution values to result in the Figure 4-13 histogram plot. Recall that the scaling factor is the standard deviation for the reduced capacity corroded wire area data shown in Figure 3-78. It is quite evident from the histogram and its statistics that 10,000 simulations are sufficient to generate the required normal distribution.

Figure 4-13. Example 1 Dispersion of Reduced Capacity Histogram.

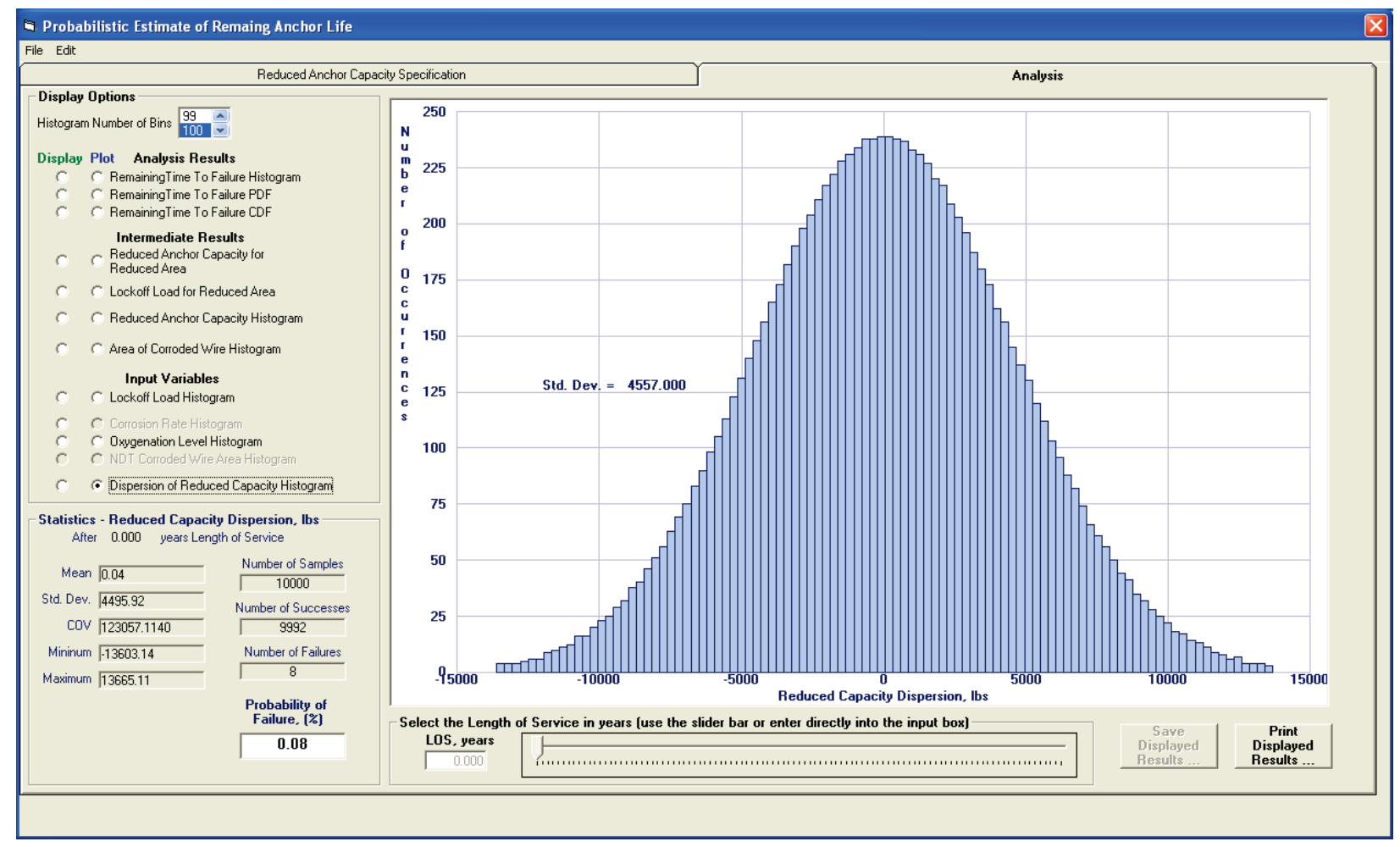




\subsubsection{Intermediate results}

The reduced anchor capacities and corroded wire area values that result from the selected corrosion variable samples are displayed with the Reduced Anchor Capacity for Reduced Area plot option. Figure 4-14 shows this scatter plot for Example 1 at an LOS of o years. Note that for an LOS time set equal to o years, no corrosion has occurred, so the corroded wire area for all points is 0.031 in. $^{2}$. Red squares indicate a sample in which the lock-off load exceeded the reduced capacity resulting in a cable failure. Blue squares indicate a sample in which the reduced capacity exceeded the lock-off load so the cable would remain intact. As the LOS is increased, the variability of the capacities and areas is observed. Figures 4-15 and 4-16 show the same plot for LOS of 10 years and 20 years, respectively. Figure 4-15 displays a reduced capacity range of 28.47 kips to 69.18 kips and a corroded wire area range of 0.0125 to 0.0275 in. ${ }^{2}$. Figure 4-16 displays a reduced capacity range of 3.14 kips to 65.30 kips and a corroded wire area range of 0.0025 to $0.025 \mathrm{in}^{2}{ }^{2}$. As expected, it is readily seen that the number of cable failure samples and the resulting POF increases with increasing LOS.

Figure 4-14. Example 1 Reduced Anchor Capacity for Reduced Area graph for LOS = 0 years.

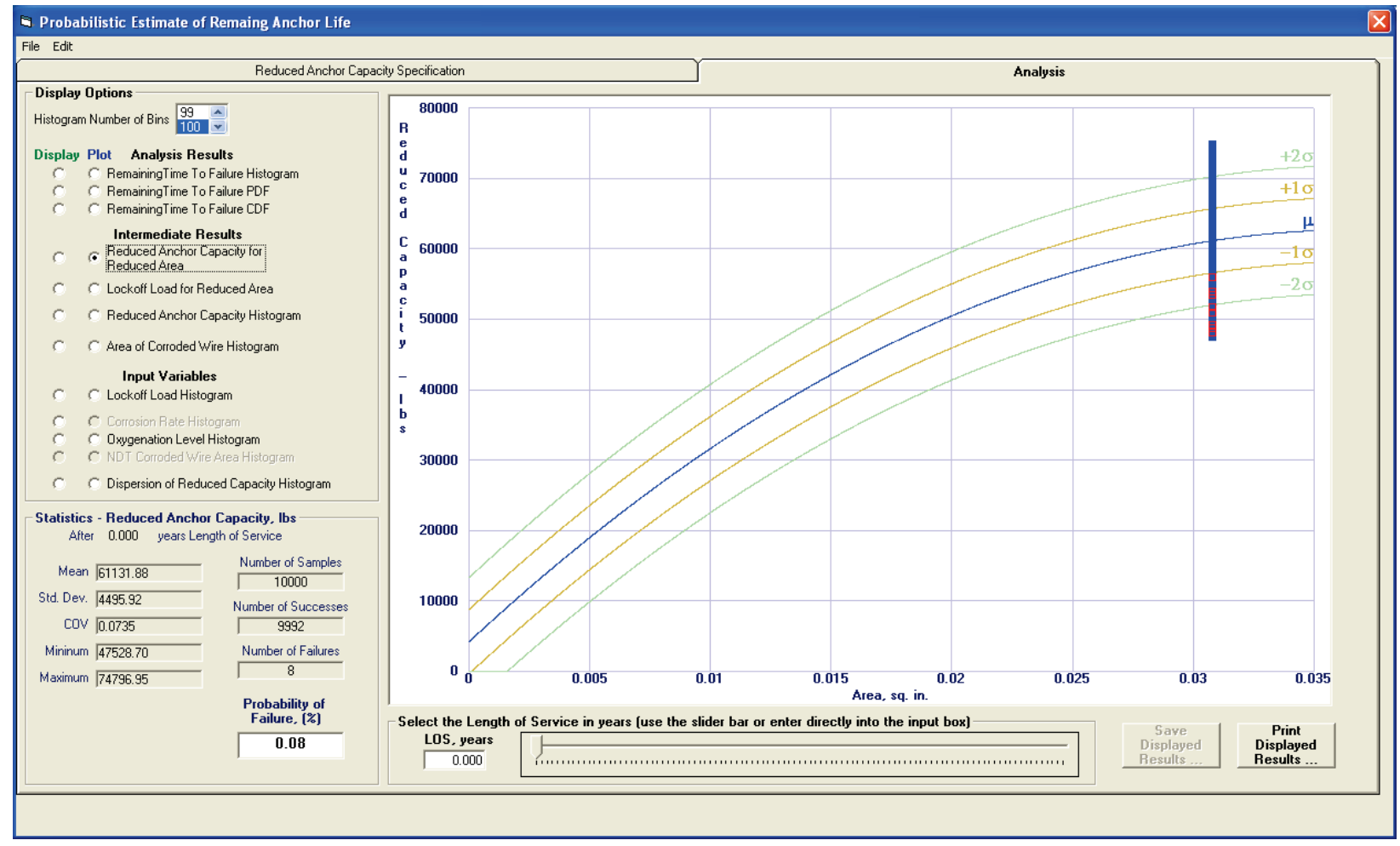


Figure 4-15. Example 1 Reduced Anchor Capacity for Reduced Area graph for LOS $=10$ years.

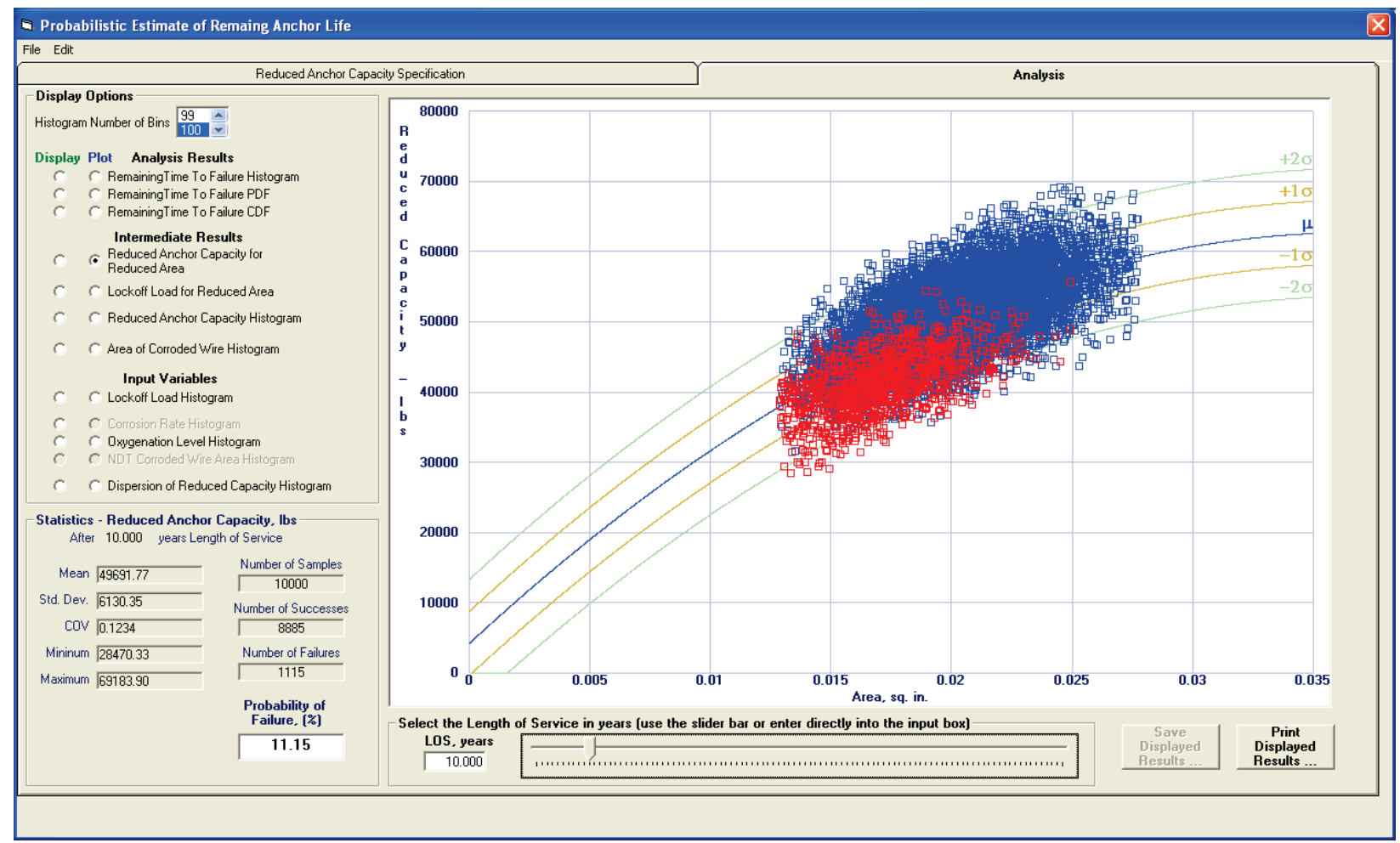

Figure 4-16. Example 1 Reduced Anchor Capacity for Reduced Area graph for LOS $=20$ years.

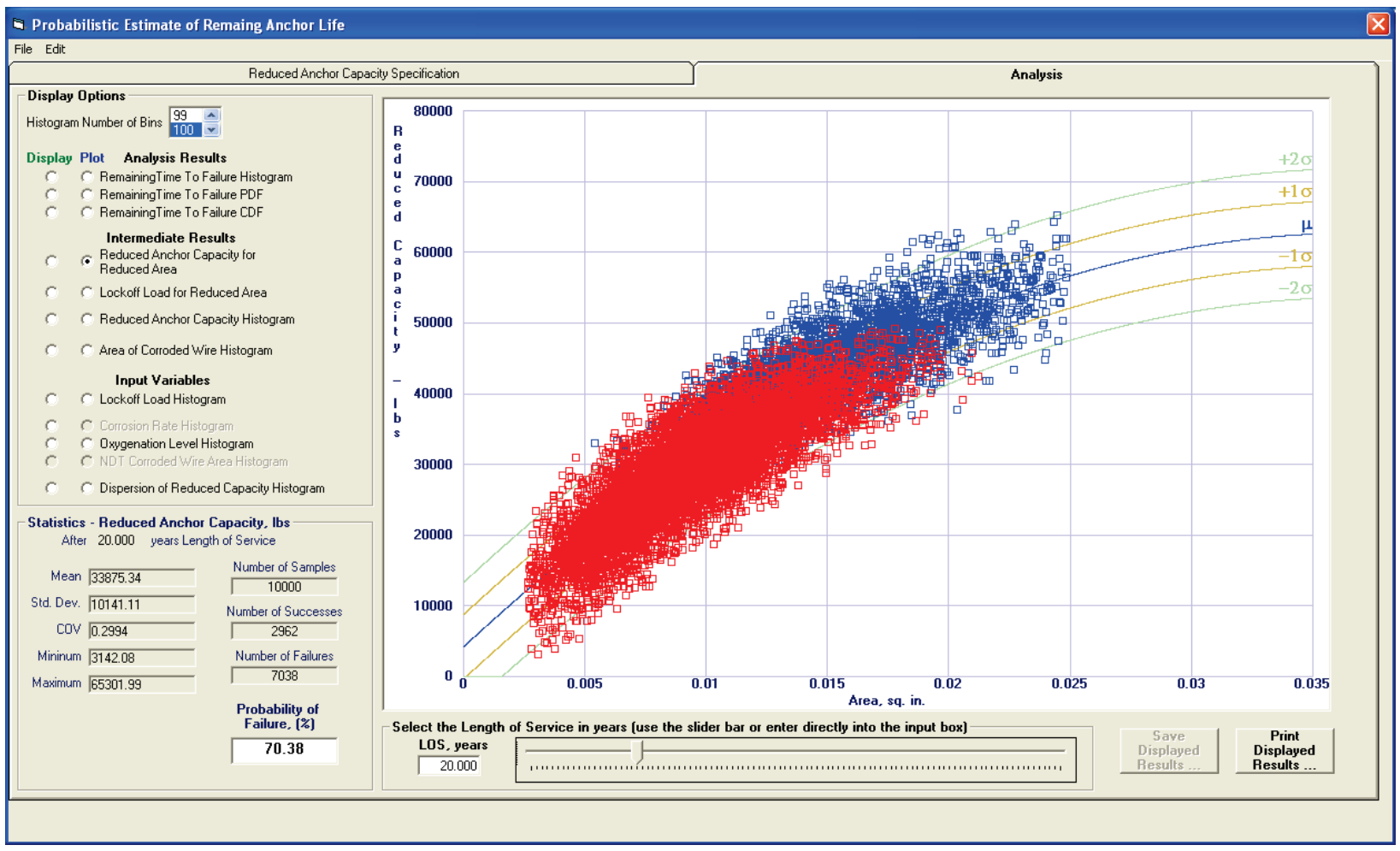


The lock-off loads and corroded wire area values that result from the selected corrosion variable samples are displayed with the Lock-off Load for Reduced Area plot option. Figure 4-17 shows this scatter plot for Example 1 at an LOS of o years. Note that for LOS $=0$ years, no corrosion has occurred, so the corroded wire area for all points is 0.031 in.2. Red squares indicate a sample in which the lock-off load exceeded the reduced capacity resulting in a cable failure. Blue squares indicate a sample in which the reduced capacity exceeded the lock-off load, so the cable would remain intact. The lock-off load is an input variable that does not vary with the LOS, so its range remains constant from 20.81 kips to 58.36 kips. As the LOS is increased, the increase in the POF is evident from the increasing red points in the graph. Figures 4-18 and 4-19 show this graph for LOS of 10 years and 20 years, respectively. The corroded wire area ranges for these figures are the same as for the reduced anchor capacity for corroded wire area plots. As expected, it is readily seen that the number of cable failure samples and resulting POF increases with increasing LOS.

Figure 4-17. Example 1 Lock-off Load for Reduced Area graph for LOS $=0$ years.

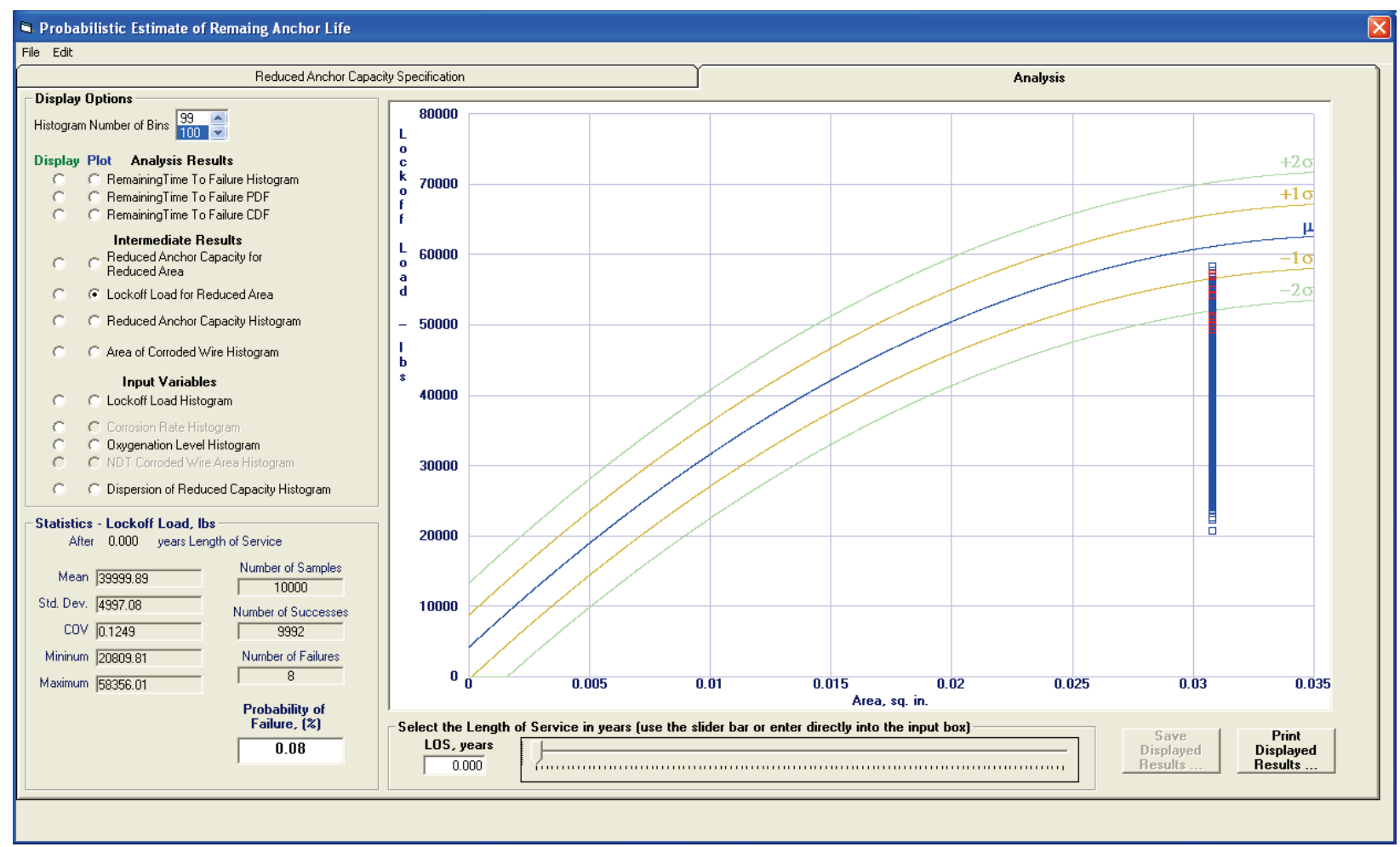


Figure 4-18. Example 1 Lock-off Load for Reduced Area graph for LOS = 10 years.

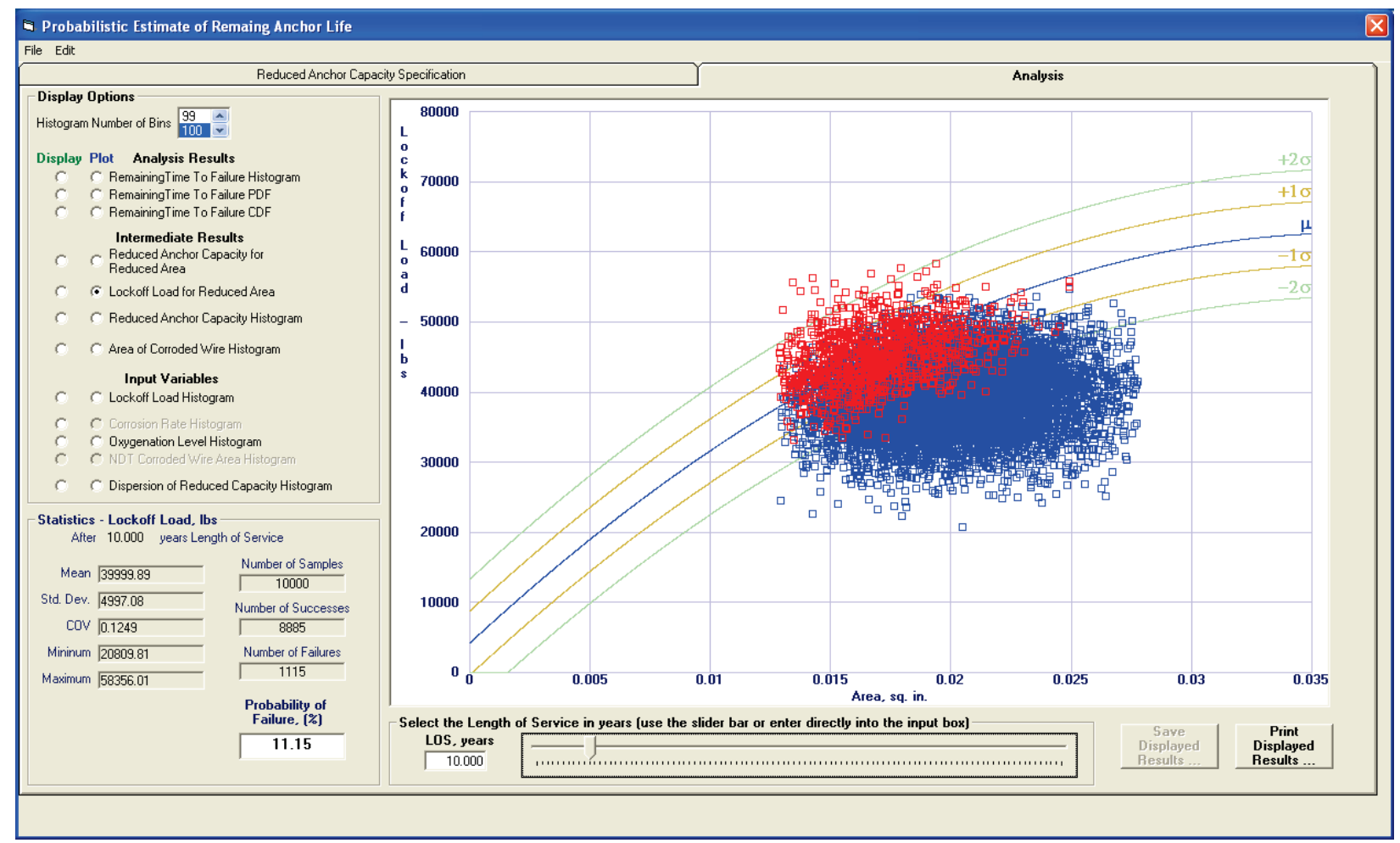

Figure 4-19. Example 1 Lock-off Load for Reduced Area graph for LOS $=20$ years.

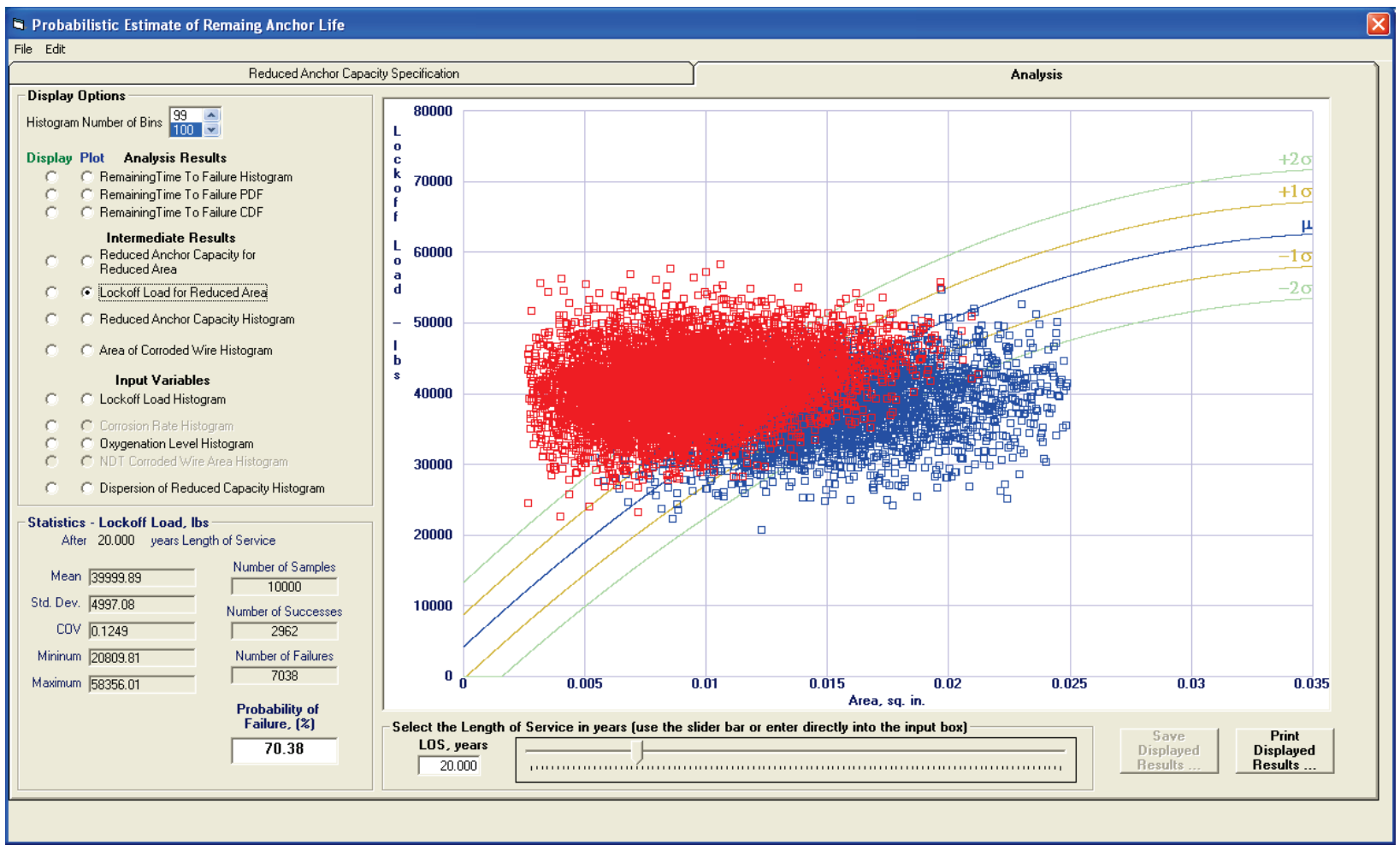


The Reduced Anchor Capacity Histogram provides a picture of the distribution of the successful reduced anchor capacities samples (those samples for which the reduced anchor capacity exceeds the lock-off load value) that result from the corrosion of the wires. The Reduced Anchor Capacity Histogram for Example 1 with an LOS of o years is shown in Figure 4-20. This figure shows a normal distribution of reduced capacities with a mean of $61.14 \mathrm{kips}$, a standard deviation of $4.49 \mathrm{kips}$ and range from 47.53 kips to $74.8 \mathrm{kips}$. As only 8 samples out of the sample set of 10,000 yielded a cable failure for this LOS, the result is a very good representation of the desired distribution.

Figure 4-21 shows the Reduced Anchor Capacity Histogram for the LOS of 20 years. This figure shows the degradation of the sample distribution due to the smaller number of successful reduced anchor capacities samples. However, an overall normal distribution is still observed, so confidence in the TTF results is accepted.

Figure 4-20. Example 1 Reduced Anchor Capacity Histogram for LOS = 0 years.

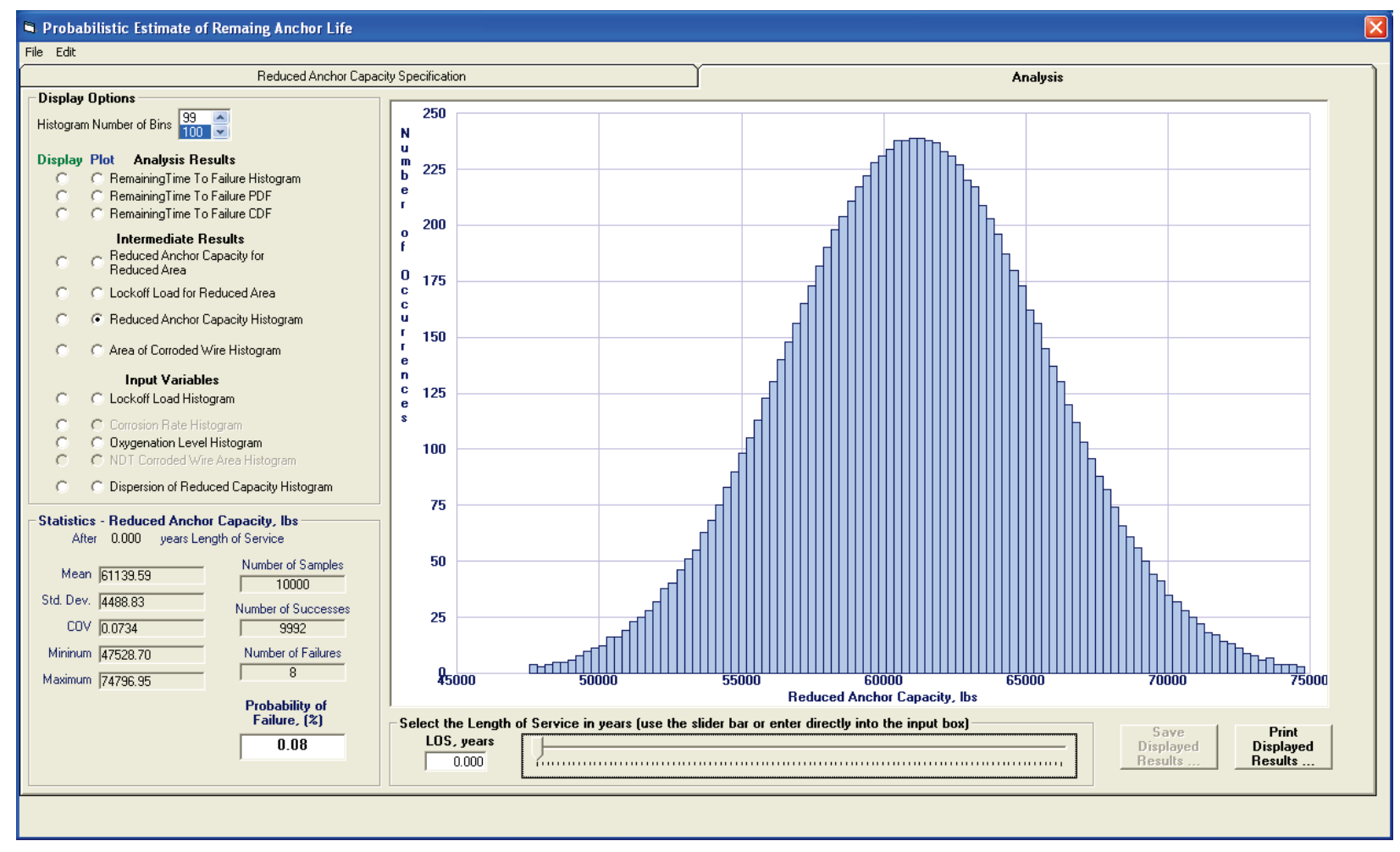


Figure 4-21. Example 1 Reduced Anchor Capacity Histogram for LOS $=20$ years.

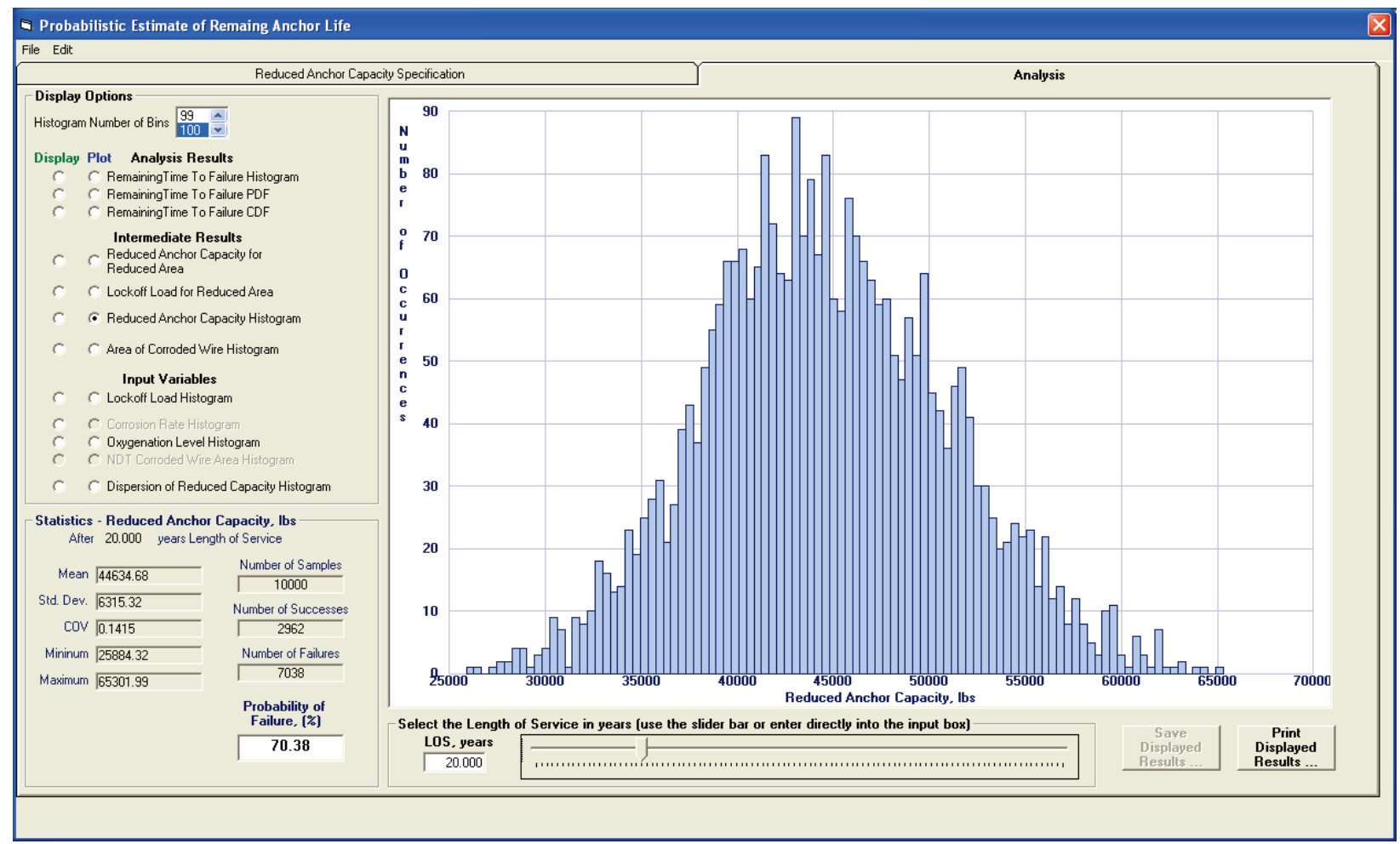

The Corroded Wire Area Histogram displays the distribution of the corroded wire samples for which the reduced anchor capacity exceeds the lock-off force. This histogram can be generated only for an LOS greater than o years. This is because no corrosion has occurred at o years, so all area samples will be a constant pristine cable area. Figure 4-22 shows this histogram for an LOS of 1 year. This histogram displays a normal distribution of values with a mean area of $0.0296 \mathrm{in.}^{2}$, a standard deviation of 0.0003 in..$^{2}$, a minimum of 0.0287 in. $^{2}$, and a maximum of 0.0305 in. ${ }^{2}$.

Figure 4-23 shows the same data histogram for an LOS of 20 years. For this histogram the number of samples utilized is 2,962, which is the number of successful samples (those for which the reduced anchor capacity exceeds the lock-off force). The distribution deviates a small amount from a normal distribution due to the lower number of samples. This is particularly true on the lower end of the corroded wire areas as the smaller area values produce fewer successful samples. However, the deviation from a normal distribution is small enough to have confidence in the TTF results. 
Figure 4-22. Example 1 Corroded Wire Area Histogram for LOS = 1 year.

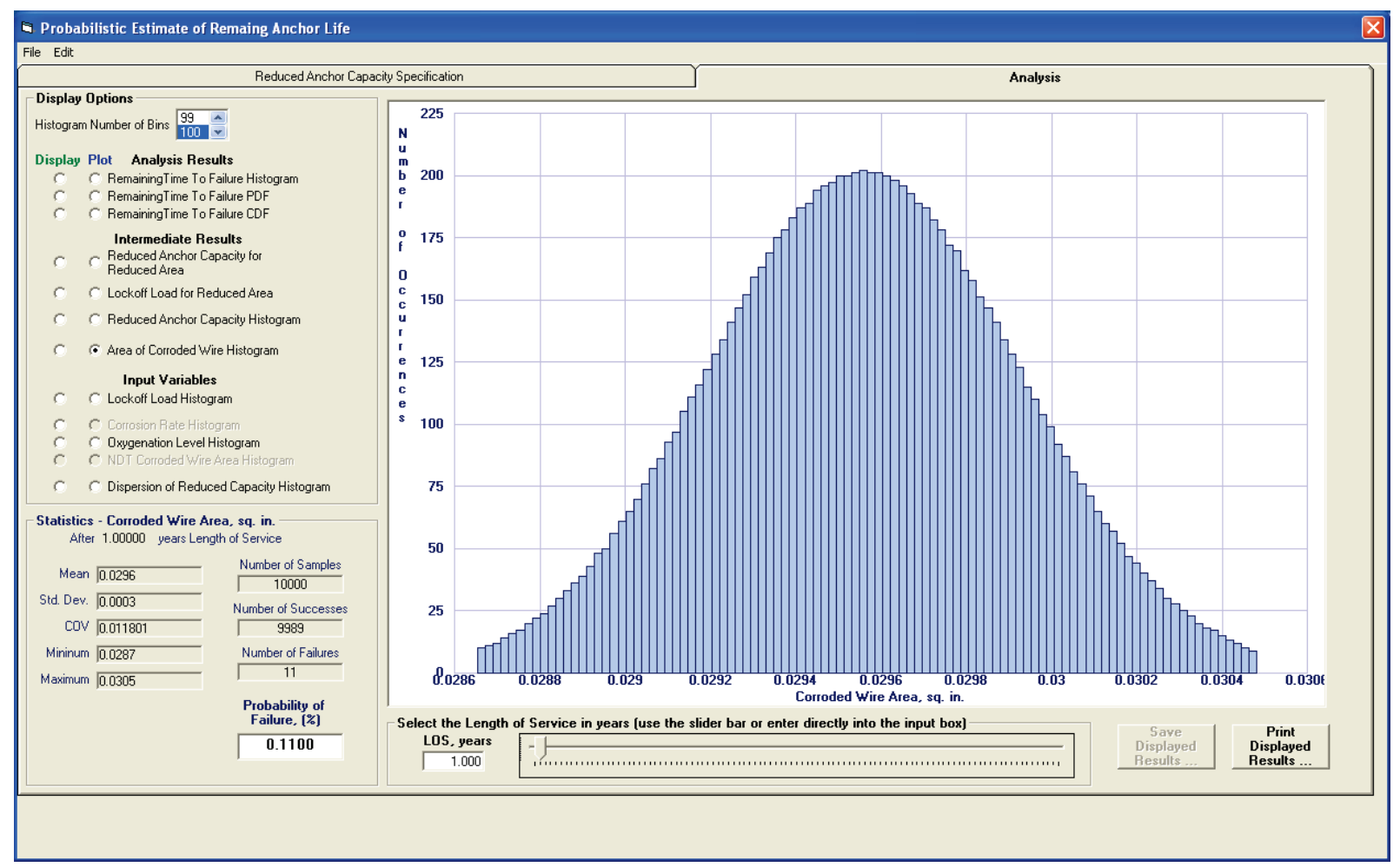

Figure 4-23. Example 1 Corroded Wire Area Histogram for LOS $=20$ years.

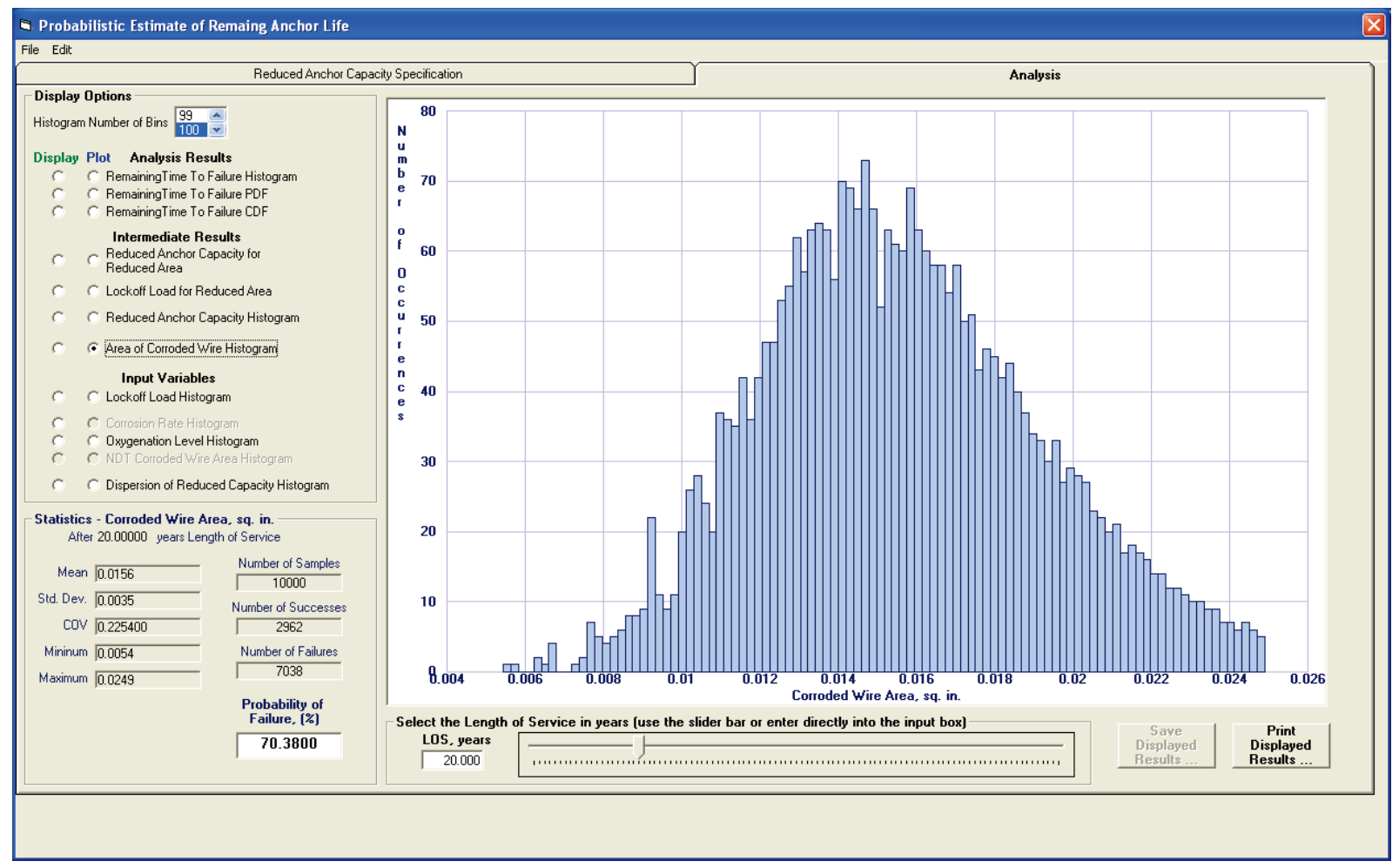




\subsection{Example 2 - Stability analysis of a lock structure}

This analysis will demonstrate the use of CAS_Stab-R to compute a probabilistic assessment of the PUP of a navigation lock wall against sliding along a user-defined slip plane and a deterministic computation of the FOS for the same structure. The probabilistic assessment of the PUP is discussed first.

\subsubsection{Probabilistic stability analysis of a lock structure}

A free body diagram of the model under analysis is shown in Figure 4-24. Due to the gap/crack shown that extends vertically from the top of the lock wall floor to the structure heel along the lock chamber face of the lock wall structure, the lock chamber hydrostatic water pressures exist at the lock wall heel. The cause of the crack was the settlement of the lock wall due to movements from the loading/unloading of the lock wall. For this reason, the diagram shows only a Structural Wedge and a Resisting Wedge. There is no

Driving Wedge as normally occurs when performing a wedge-based stability analysis as there is in the example problem in Appendix B of this report. A linear change in total head, $H$, occurs along the slip plane from elevation $85 \mathrm{ft}$ to elevation $24 \mathrm{ft}$ at the end of the slip plane (node 23 in Figure 4-26).

Figure 4-24. Lock wall model free body diagram of Example 2.

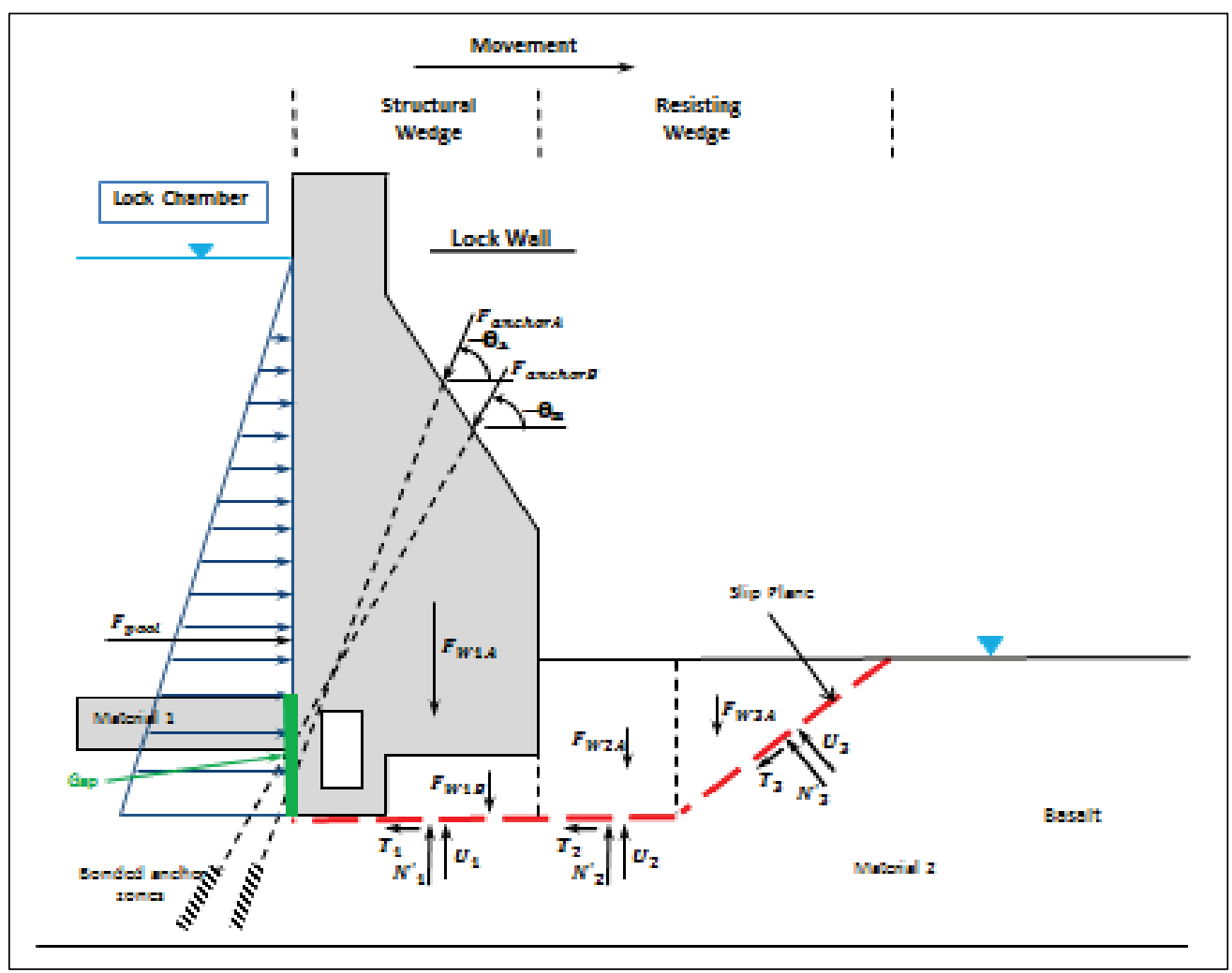


The CAS_Stab-R model (lock wall and surrounding topography) for this example is shown in Figure 4-24. This model consists of a concrete lock wall monolith embedded in a basalt rock foundation with a potential slip plane. The lock chamber has a concrete floor on top of the basalt. Two prestressed anchorage systems provide lock wall reinforcement.

The node numbers and assigned coordinates that are used to define the model geometry are shown in Table 4-1. The model with the node numbers displayed is shown in Figure 4-26. The Structure tab on the Geometry tab (seen in Figure 4-25) was used to define the lock wall structure and gallery. The structure was defined by clicking the Select Concrete Structure Nodes button. The nodes that define the structure are 1, 2, 14, 22, 3, 4, 5, and 6 in the listed order. The gallery style was selected as rectangular with width of $6 \mathrm{ft}$ and height of $8 \mathrm{ft}$. Node 7 was selected as the location of the bottom center of the gallery. As this structure has no gallery drain, the gallery drain selection was NO DRAIN.

Figure 4-25. Navigation lock wall model of example problem.

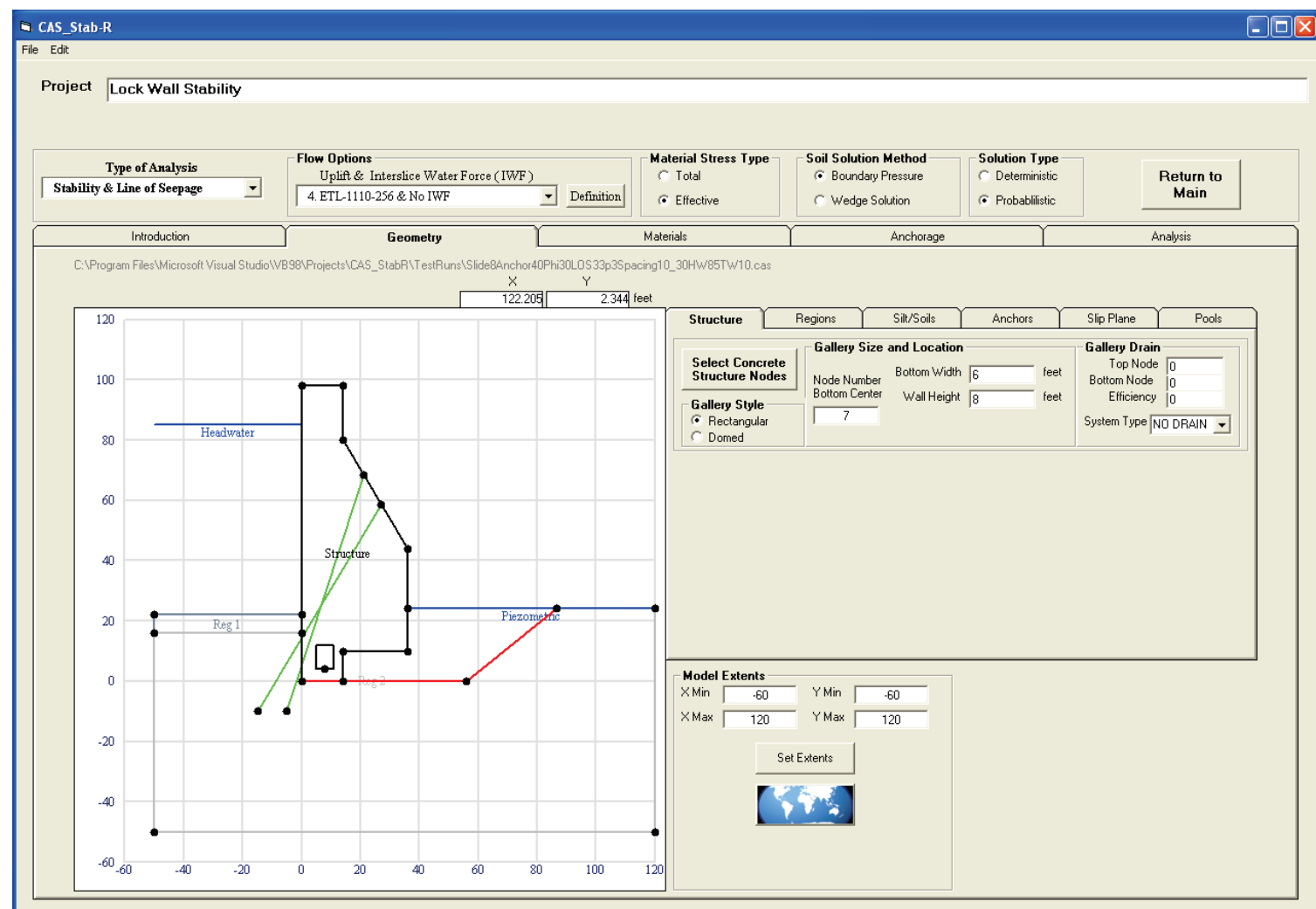


Table 4-1. Lock wall structure nodes.

\begin{tabular}{|c|c|c|}
\hline Node Number & $x$-Coordinate & $y$-Coordinate \\
\hline 1 & 0 & 0 \\
\hline 2 & 14 & 0 \\
\hline 3 & 36 & 44 \\
\hline 4 & 14 & 80 \\
\hline 5 & 14 & 98 \\
\hline 6 & 0 & 98 \\
\hline 7 & 8 & 4 \\
\hline 8 & -50 & 16 \\
\hline 9 & 0 & 16 \\
\hline 10 & 0 & 22 \\
\hline 11 & 50 & 22 \\
\hline 12 & -50 & -50 \\
\hline 13 & 120 & -50 \\
\hline 14 & 14 & 10 \\
\hline 15 & 120 & 24 \\
\hline 16 & 36 & 24 \\
\hline 17 & 56 & 0 \\
\hline 18 & 27.14851 & 58.484256 \\
\hline 19 & 21.013872 & 68.522755 \\
\hline 20 & -5 & -10 \\
\hline 21 & -15 & -10 \\
\hline 22 & 36 & 10 \\
\hline 23 & 86.545455 & 24 \\
\hline
\end{tabular}

The rock regions were specified using the Add a Region button on the Regions tab of the Geometry tab. This button is shown in Figure 4-27. Rock Region 1 consists of nodes 8, 9, 10, and 11 in the listed order. Rock Region 2 consists of nodes 12, 13, 15, 16, 22, 14, 2, 1, 9, and 8 in the listed order. Rock Region 1 is the concrete lock chamber floor, and Rock Region 2 is the foundation basalt rock. 
Figure 4-26. Model geometry with displayed node numbers.

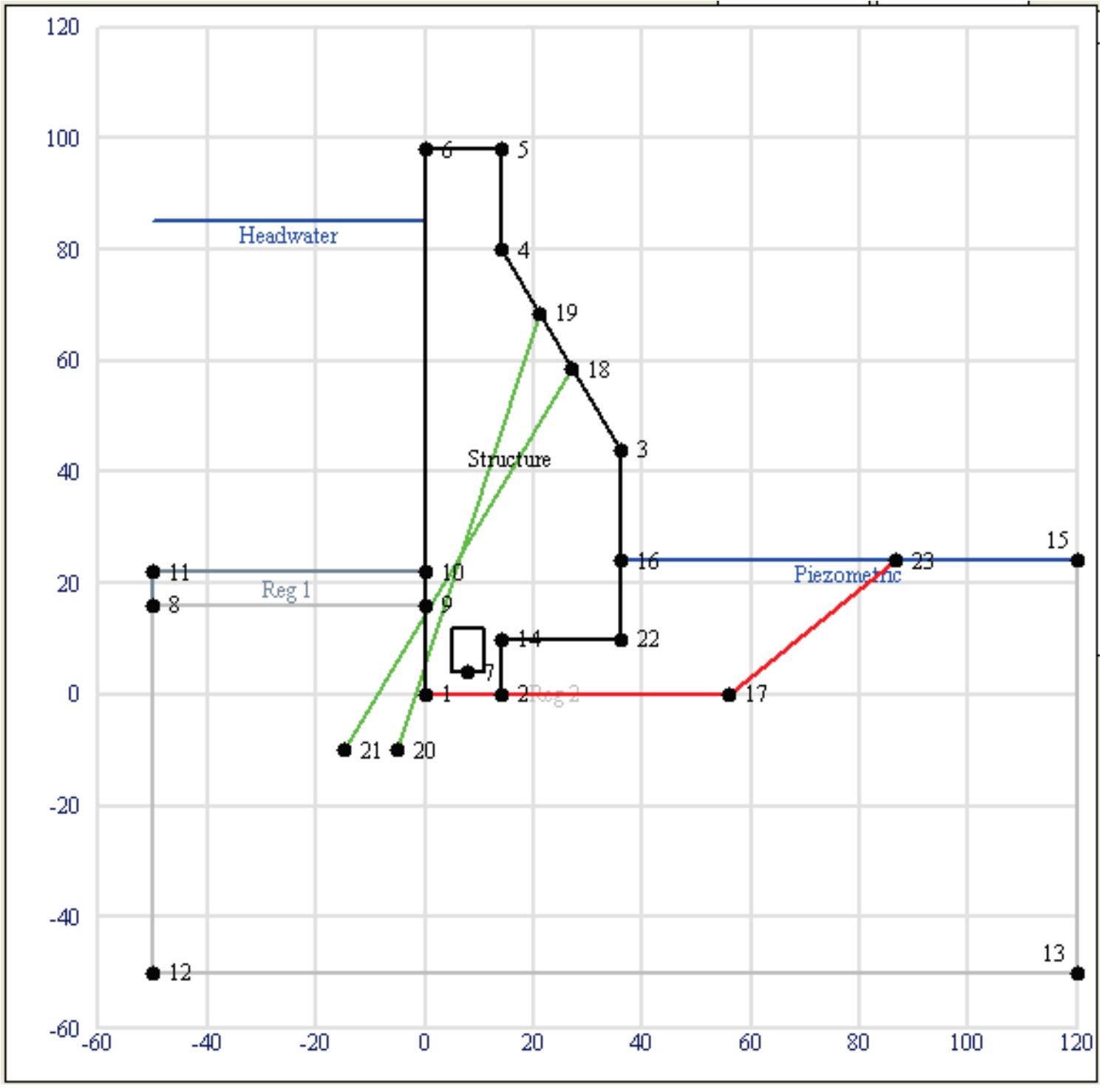

Two materials are defined (Figure 4-27). The first material is concrete used in the structure and the lock floor region. The necessary properties of the concrete are the densities and unit weight. These three properties are set to $150 \mathrm{lb} / \mathrm{ft} 3$. The properties assigned to Material 1 are shown in Figure 4-28. The second material is the foundation basalt rock. The necessary properties of the basalt rock are the densities, unit weight, and internal friction angle, $\phi$. These densities and unit weight are set to $160 \mathrm{lb} / \mathrm{ft}^{3}$. The internal friction angle is set to 30 degrees (deg). The properties assigned to Material 2 are shown in Figure 4-29. These figures also show the mapping of the materials to the structure and the two rock regions. The Region - Material Mapping box shows that the structure is assigned Material 1, Region 1 is assigned Material 1, and Region 2 is assigned to Material 2. 
Figure 4-27. Regions tab.

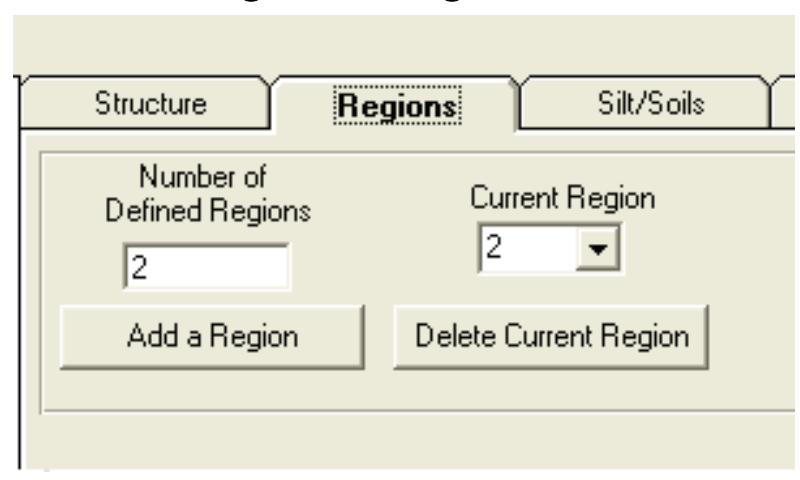

Figure 4-28. Materials tab showing the properties of Material 1.

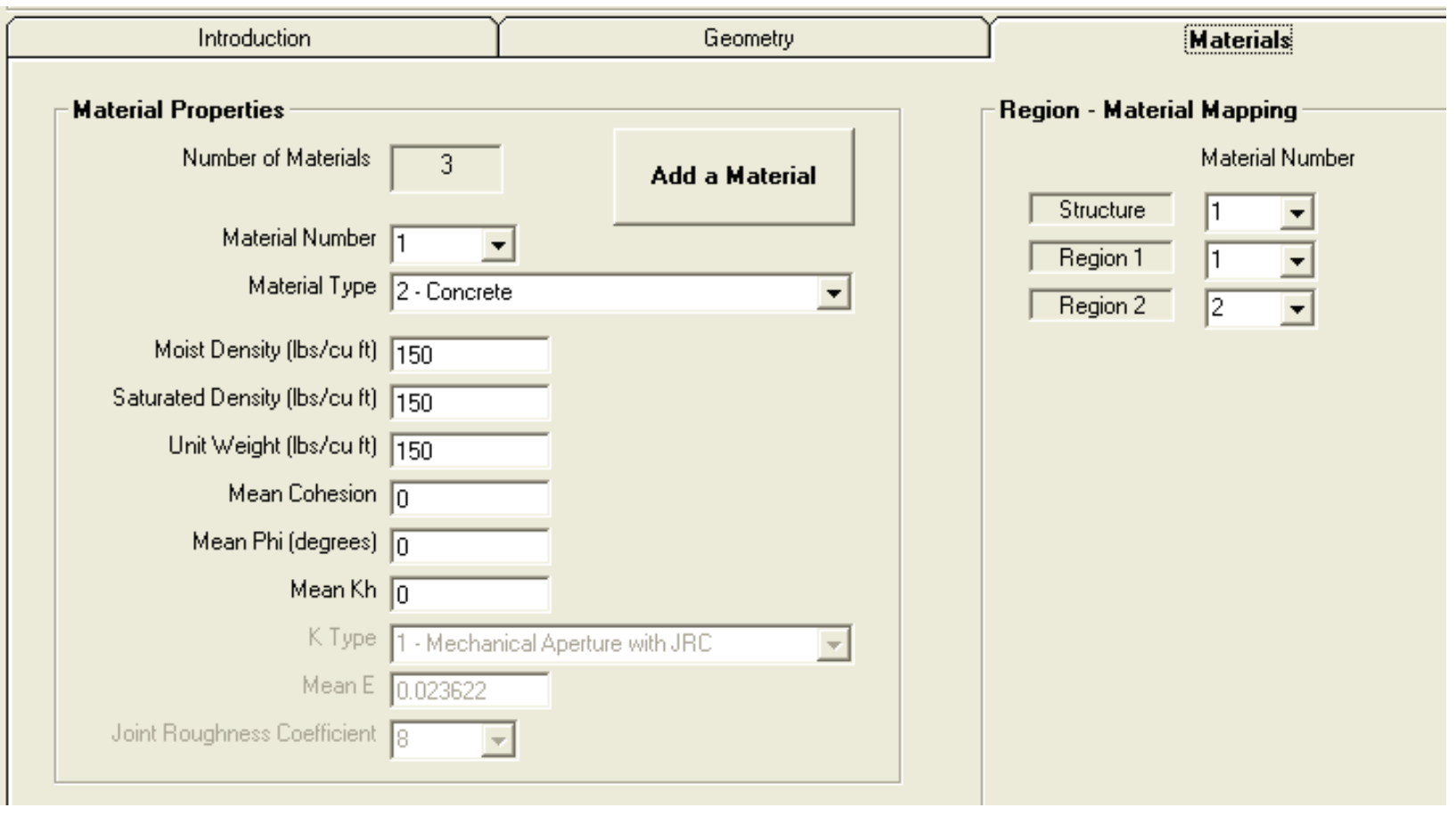

The slip plane is defined by nodes 1,17 , and 23 in the listed order. The slip plane definition is made by using the Select Slip Plane Nodes button on the Slip Plane tab of the Geometry tab.

Two anchor locations are defined using the Anchors tab of the Geometry tab as shown in Figure 4-30. Anchor 1 extends from node 20 at the bottom to the top node of 19. Anchor 2 extends from node 21 at the bottom to the top node of 18 . The forces applied to the anchors are discussed in later sections. 
Figure 4-29. Materials tab showing the properties of Material 2.

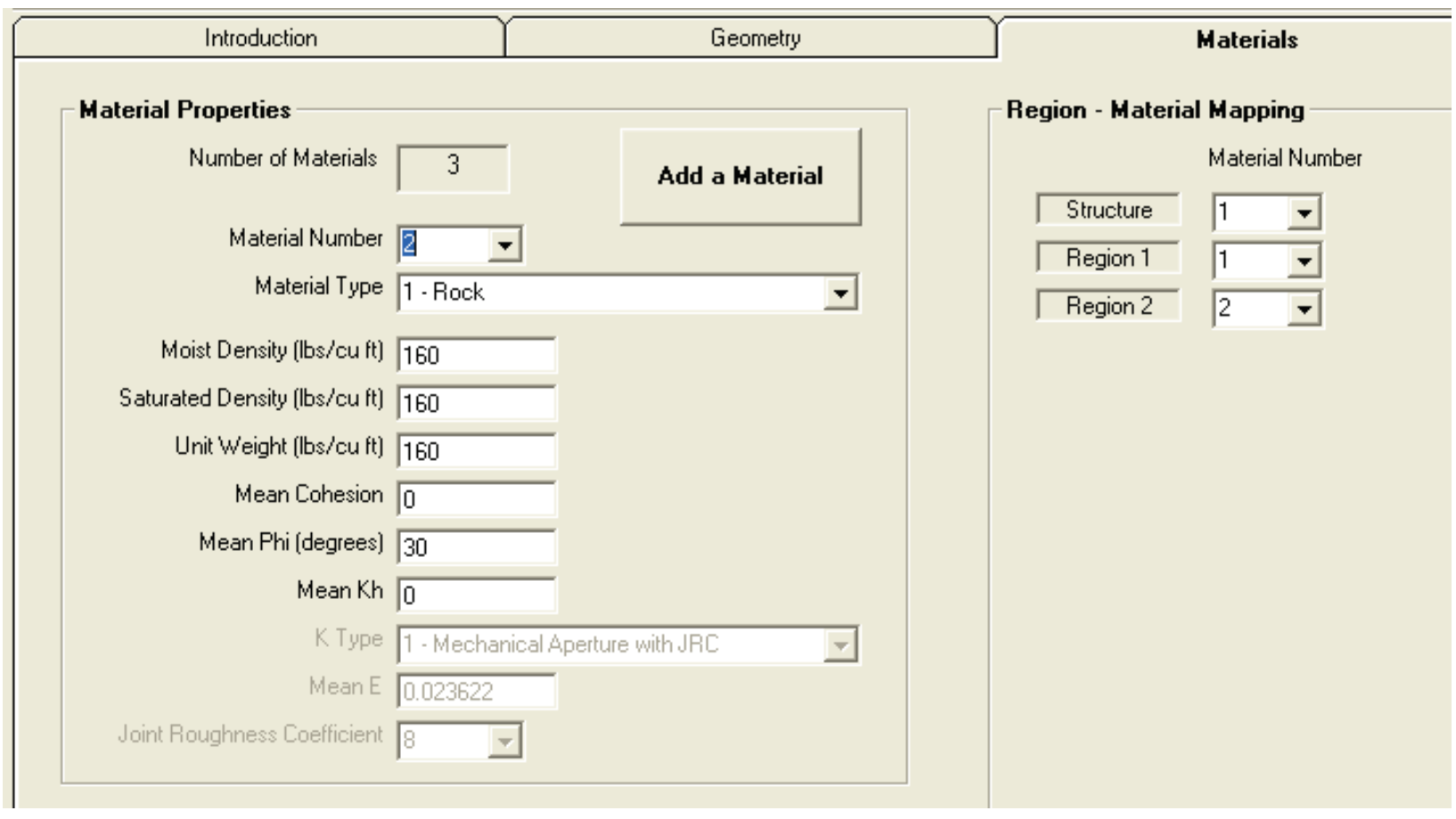

Figure 4-30. Anchors tab-Definition of lock wall anchors for the example problem.

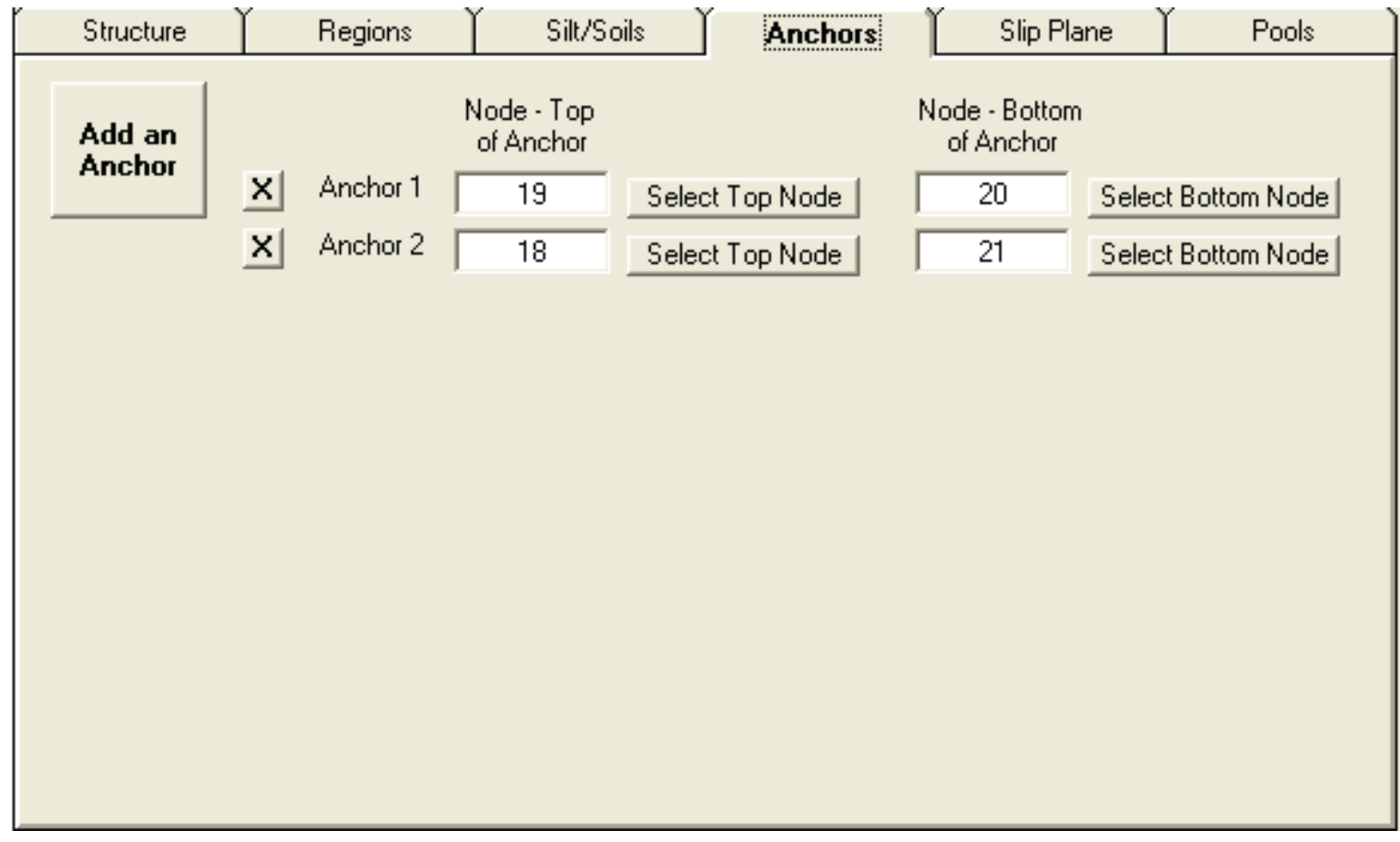

The chosen headwater and tailwater elevations are $85 \mathrm{ft}$ and $24 \mathrm{ft}$, respectively, for this example. Figure 4-31 shows the Pools tab where these elevations are assigned. This tab also shows the density of water setting of $62.4 \mathrm{lb} / \mathrm{ft}^{3}$. 
Figure 4-31. Pools tab-Definitions for the example problem.

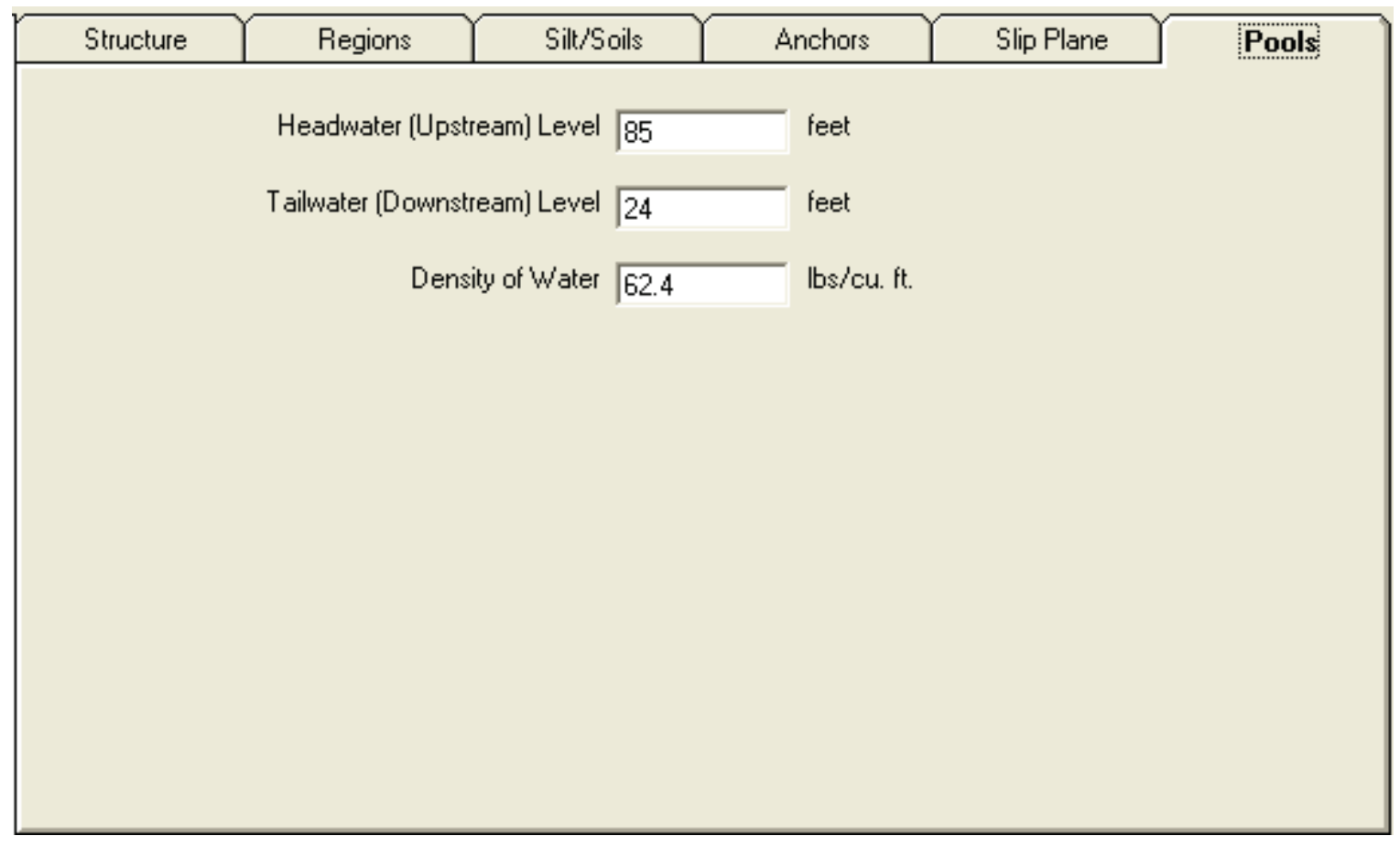

The lock-off force per cable in an anchor bundle is assigned to be $41,113 \mathrm{lb}$ for both anchorages. This value is two-thirds of the mean capacity for a pristine seven-wire cable as identified in Ebeling et al. (2016). Both anchorages are assigned to be grouped into bundles of 30 cables with a spacing of $10 \mathrm{ft}$ between anchors. The LOS time chosen was 27 years and 6 months. The LOS was chosen by a method of trial and error until a PUP of 0.95 was the result of the simulation. The method of computing the loss of material in each cable over time was chosen as the oxygenation level in the anchor environment with a mean value of $1.175 \mathrm{ppm}$. CAS_Stab-R utilizes the oxygenation level to corrosion rate relationship provided in Ebeling et al. (2016). This relationship is viewed by clicking the Oxygenation Curve button on the Anchorage tab as shown in Figure 4-32. The capacity of the corroded cable determination is chosen to be the Corroded Wire Area option. This utilizes the relationship of the corroded wire area to the remaining cable capacity as presented in Ebeling et al. (2016). The curve showing this relationship is displayed in the lower left corner of the Anchorage tab. The entry of the setup information detailed in this paragraph is made on the Anchorage tab and is shown in Figure 4-33. Figure 4-33 shows the information for Anchor 1. Anchor 2 is set to the same lock-off force, grouping, and spacing values. 
Figure 4-32. Anchorage tab-Corrosion rate - Oxygenation level curve from Ebeling et al. (2016).

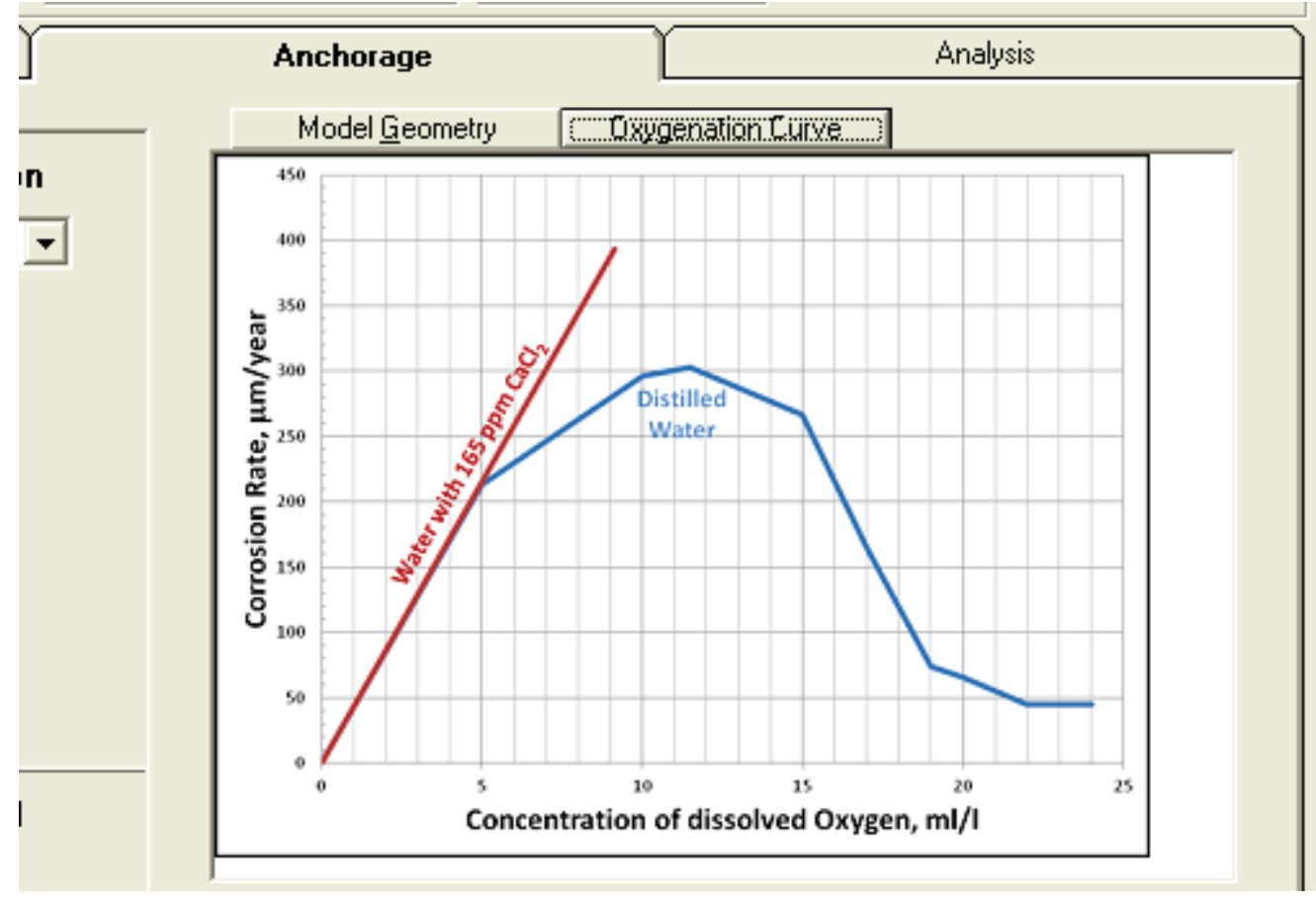

Figure 4-33. Anchorage tab with example problem selections for Anchor 1.

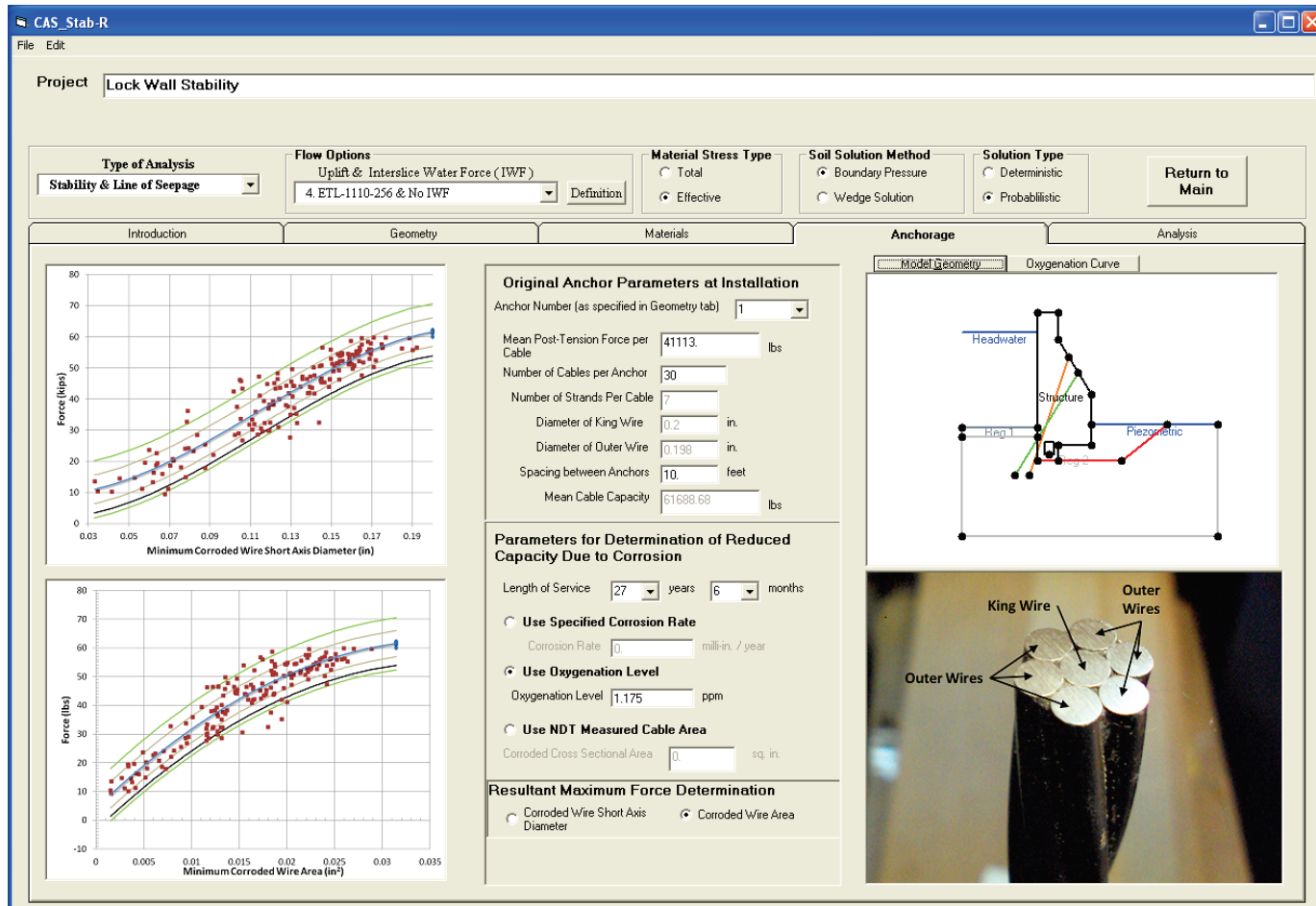


The variables for this simulation are the lock-off load forces for the two anchors, the oxygenation level, and the internal friction angle, $\phi$, for Material 2. Prior to setting the distribution parameters for these variables, the Probabilistic Solution Type must be selected as seen in Figure 4-33. These variable distribution values are assigned on the Probability Variable Input window. To access this window, click the Edit-Probability Distributions menu option as shown in Figure 4-34.

Figure 4-34. Probability Distributions menu option.

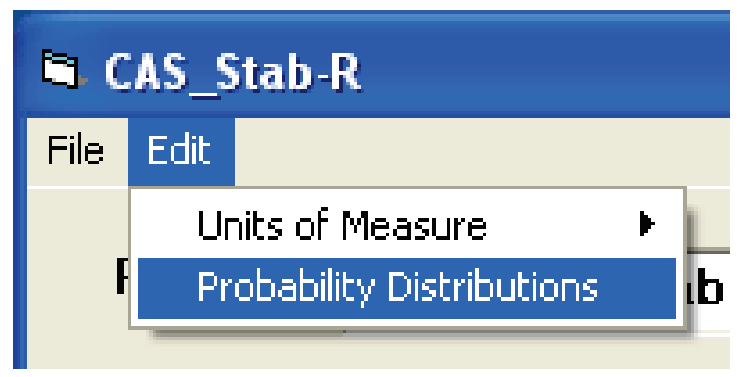

For this example, the oxygenation level variable is assigned a normal distribution with a mean value of $1.175 \mathrm{ppm}$ with a standard deviation of $0.3525 \mathrm{ppm}$, which yields a $\mathrm{CoV}$ of 0.3. The Probability Variable Input window for this setting is shown in Figure 4-35.

The initial lock-off force for Anchor 1 is assigned a normal distribution with a mean value of $41,113 \mathrm{lb}$ with a standard deviation of 2,055.65 lb, which yields a CoV of 0.05. The Probability Variable Input window for this setting is shown in Figure 4-36. The initial lock-off force for Anchor 2 is assigned the same distribution of values.

The internal friction angle, $\phi$, is assigned a normal distribution with a mean value of $30 \mathrm{deg}$ with a standard deviation of $3 \mathrm{deg}$, which yields a $\mathrm{CoV}$ of 0.1. The Probability Variable Input window for this setting is shown in Figure 4-37. 
Figure 4-35. Probability distribution for Oxygenation Level variable.

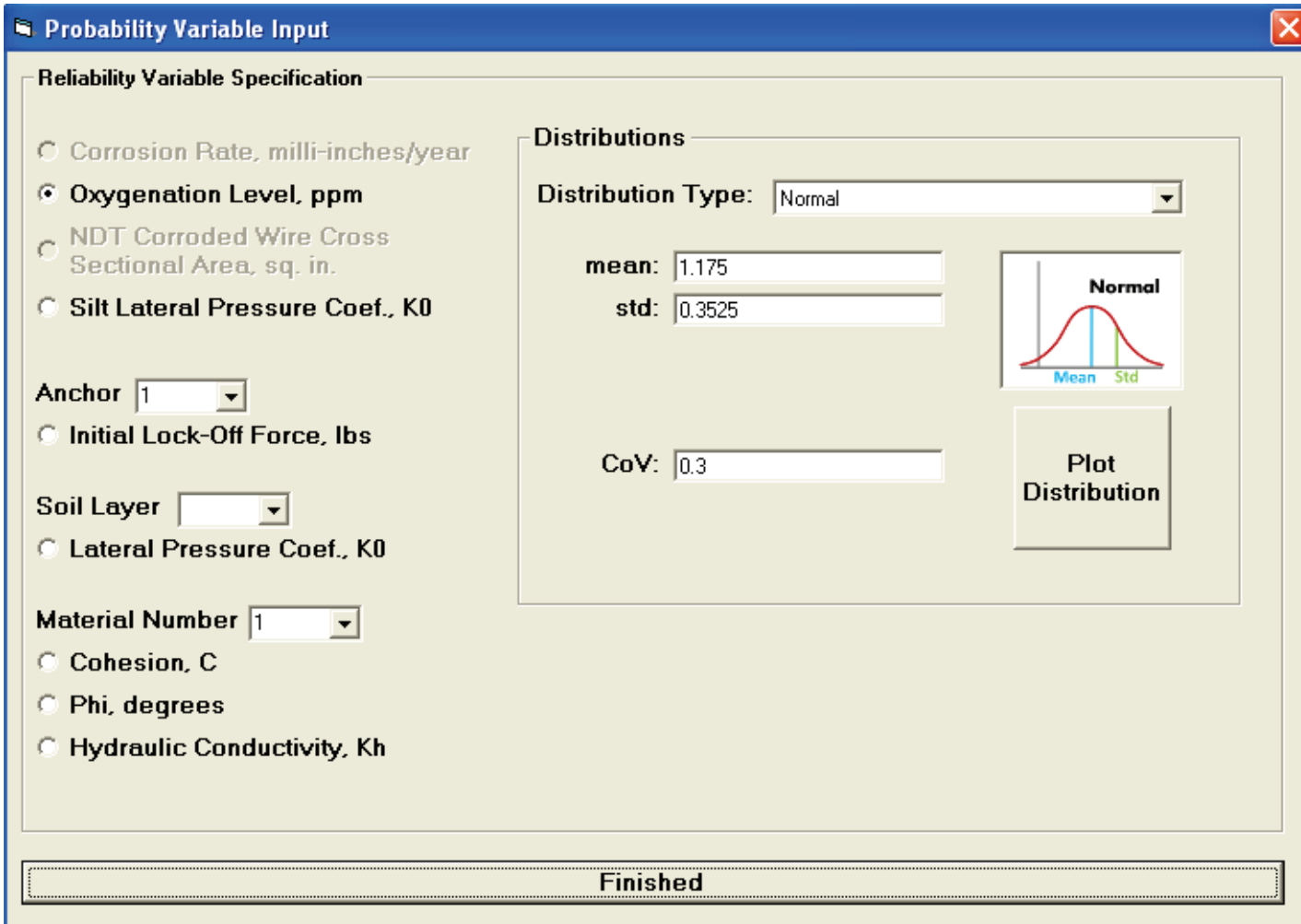

Figure 4-36. Probability distribution for Anchor 1 lock-off force variable.

Probability Variable Input

Reliability Variable Specification

$\Upsilon$ Corrosion Rate, milli-inches/year

Distributions

C Oxygenation Level, ppm

c NDT Corroded Wire Cross Sectional Area, sq. in.

C Silt Lateral Pressure Coef., K0

Anchor $1 \quad \square$

c Initial Lock-Off Force, Ibs

Soil Layer $\square$

C Lateral Pressure Coef., K0

Material Number $1 \quad$ -

$C$ Cohesion, $\mathrm{C}$

C Phi, degrees

C Hydraulic Conductivity, $\mathbf{K h}$

Distribution Type: Normal mean: 41113

std: 2055.65

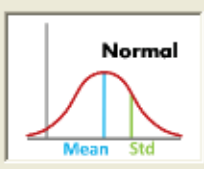

Cov: 0.05

Plot Distribution

Finished 
Figure 4-37. Probability distribution for the Material Number 2 internal friction angle, $\phi$.

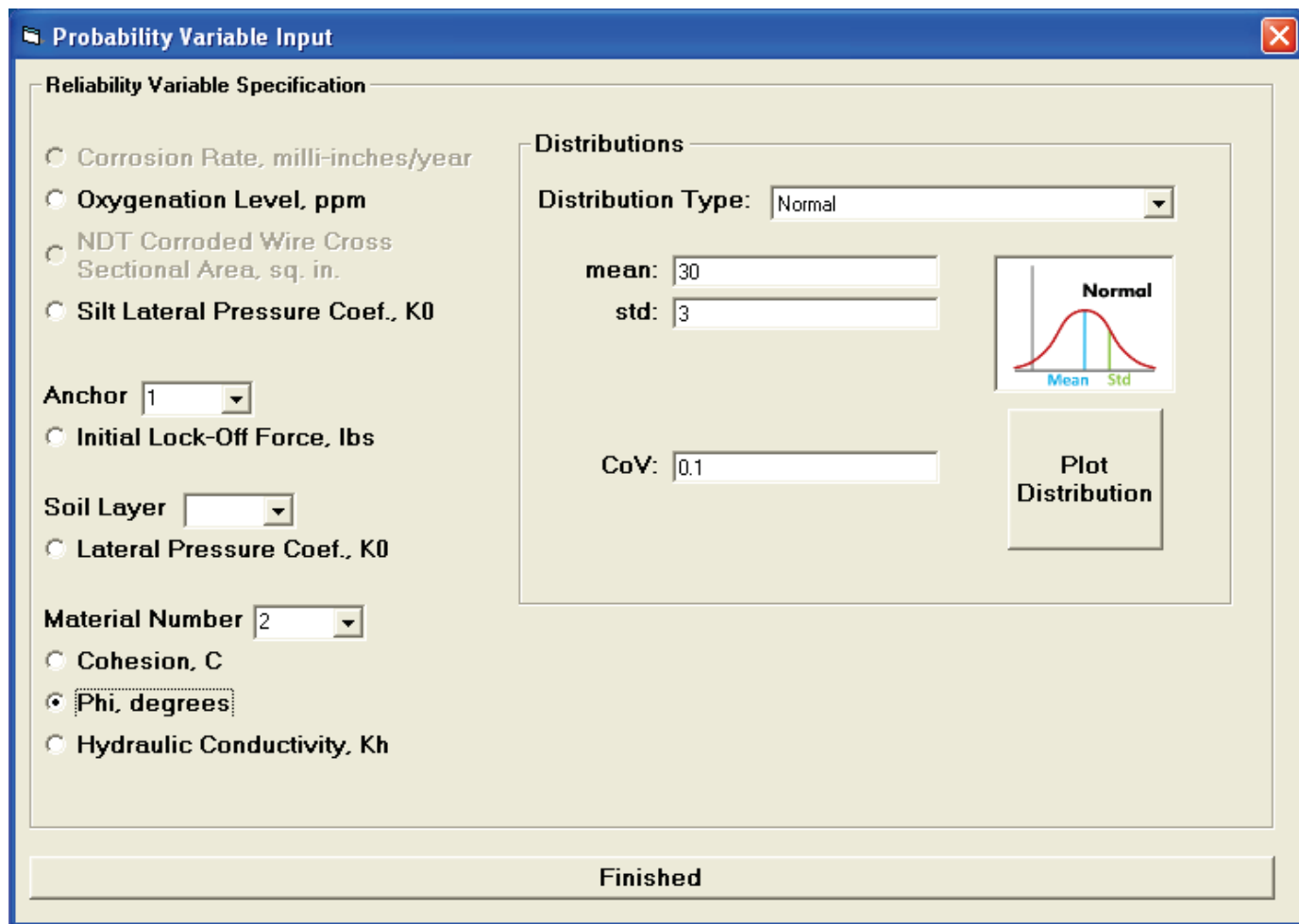

To perform the analysis, select the Analysis tab as shown in Figure 4-38. The desired parameters in the Iteration and Simulation Parameters frame must be addressed prior to executing the simulation. For this example the Uplift Force Tolerance is assigned $100 \mathrm{lb}$. For each sample in the simulation, an iterative process is used to determine the FOS, which results in an imbalance force less than the Uplift Force Tolerance. The imbalance force is determined according to the wedge solution method presented in ETL 1110-2-256 (HQUSACE 1981). If the imbalance force computed for the sample does not converge to less than the Uplift Force Tolerance setting within a maximum number of iterations, the FOS returned is 0.05. The maximum number of iterations is assigned in the Maximum Number of Iterations for Solution Convergence input box. This value is assigned a value of 100 for this example. The Number of Samples in Simulation setting determines the number of Dakota-generated LHS desired for the simulation. The user must choose a number of samples to ensure an adequate variable distribution. A comparison of PUP values determined for a varying number of samples is presented in section 4.3.3. For this example, the number of samples is assigned 5,000. 
Figure 4-38. Initial Analysis tab.

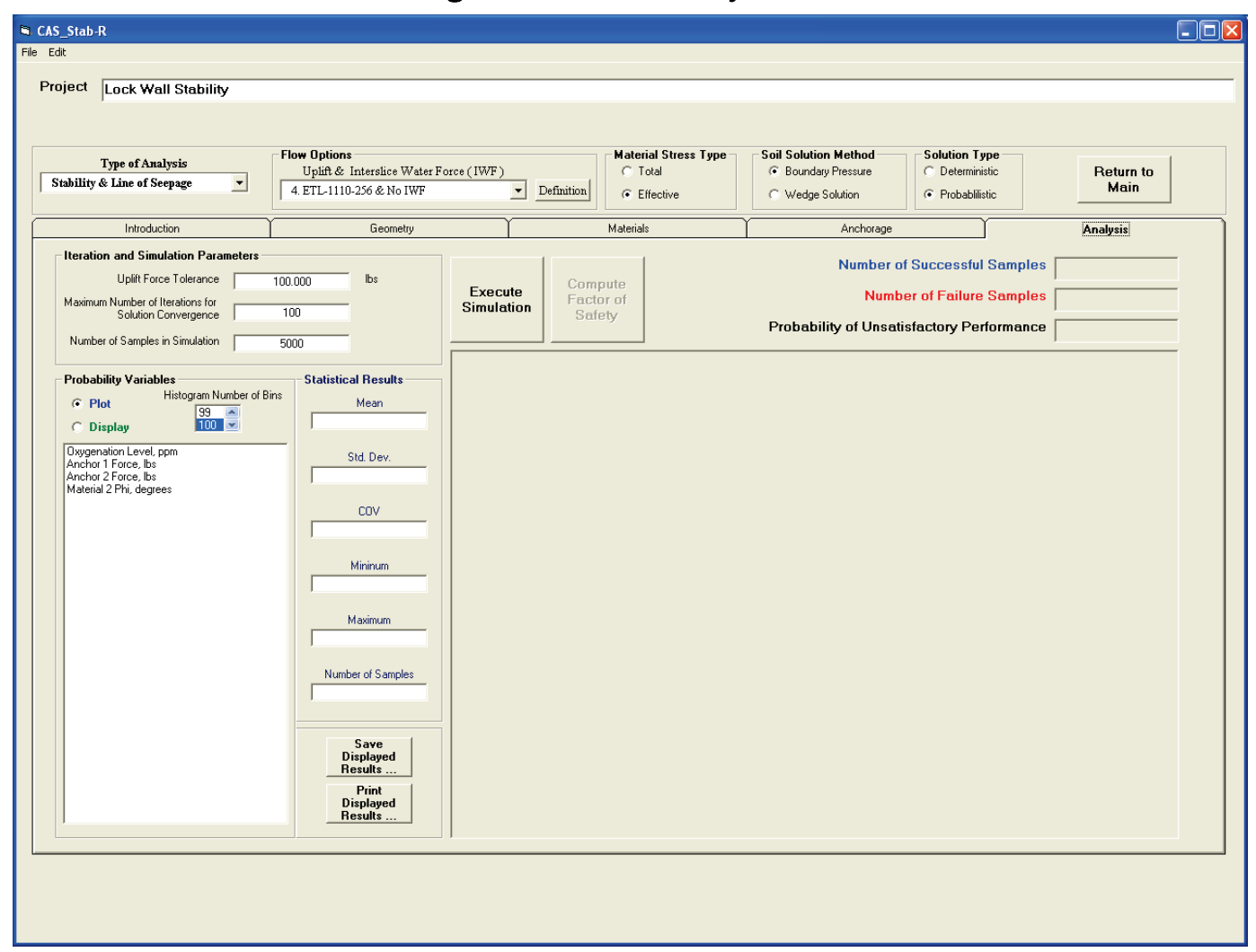

Clicking the Execute Simulation button will begin the simulation process. The first step in the simulation is the determination of the optimum design point about which Dakota software will center the distribution of variables for importance sampling. This is determined using the ASM computation method presented in Ebeling et al. (2013). A window is displayed as shown in Figure 4-39 for the duration of this step to alert the user to the ongoing process. For this example, the only variable affected is the Material $2 \mathrm{Phi}$ mean value. The mean value of Phi for importance sampling is $26.7 \mathrm{deg}$ as evidenced by the distributions presented in the following report section 4.3.2. The simulation process then proceeds to the determination of PUP. During this step, a window with a progress bar is displayed as shown in Figure 4-40. At the conclusion of the simulation process, the display boxes for the Number of Successful Samples, the Number of Failure Samples, and Probability of Unsatisfactory Performance are populated with the results of the simulation using importance sampling as shown in Figure 441. For this example, the Number of Failure Samples was 4,745 out of the total of 5,000 samples, which yields a PUP of 0.949 . 
Figure 4-39. Determining ASM Design Point window.

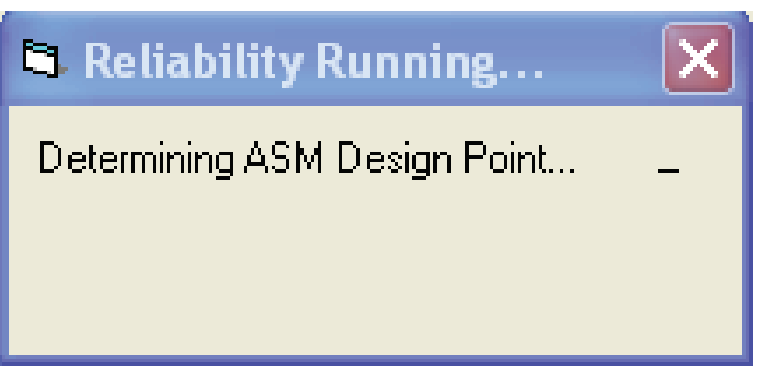

Figure 4-40. Running Simulation window.

\section{E. Reliability Running... X}

\section{Running Simulation...}

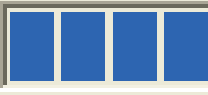

Figure 4-41. Analysis tab showing the results of the example problem simulation.

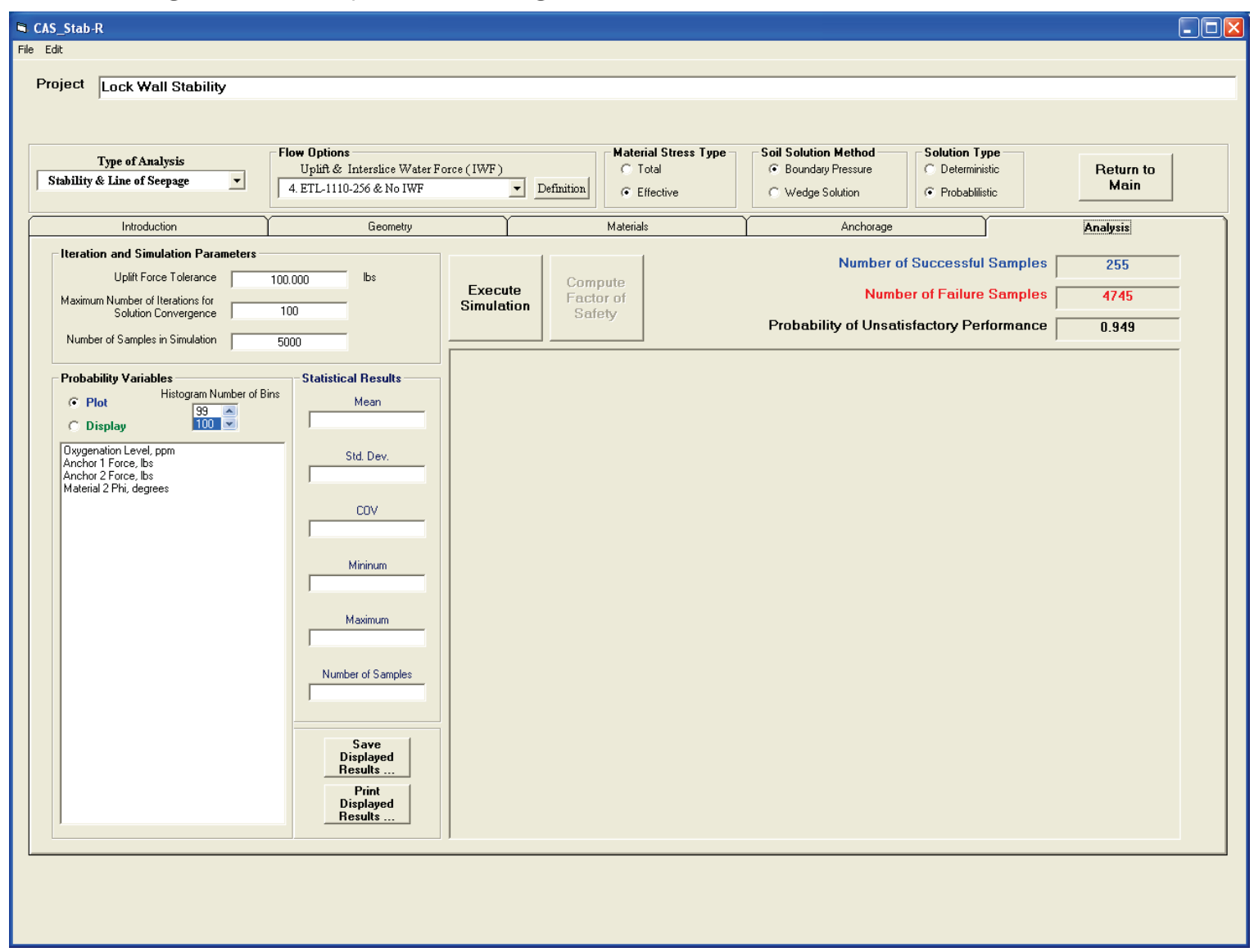




\subsubsection{Quality of dataset variables - Stability of a lock wall structure example}

The variables in the dataset for this example are shown in the list box in the Probability Variables frame in Figure 4-38 above. The Dakotagenerated distribution of values and statistics for each variable is viewed by clicking on the variable in the list box. Recall that these distributions are centered about the ASM design point for importance sampling. The sample values from these distributions are used to derive the actual PUP values from the user-defined distributions. These distribution plots are provided to show the qualitative results of the sampling process for importance sampling at the ASM design point.

Clicking on the Oxygenation Level variable shows the results in Figure 4-42. The histogram shows a normal distribution of values, and the statistics frame shows the distribution is centered at the mean value of $1.175 \mathrm{ppm}$ with a standard deviation of $0.3524 \mathrm{ppm}$. These values match the distribution specified in the Probability Variable Input window.

Figure 4-42. Oxygenation Level distribution.

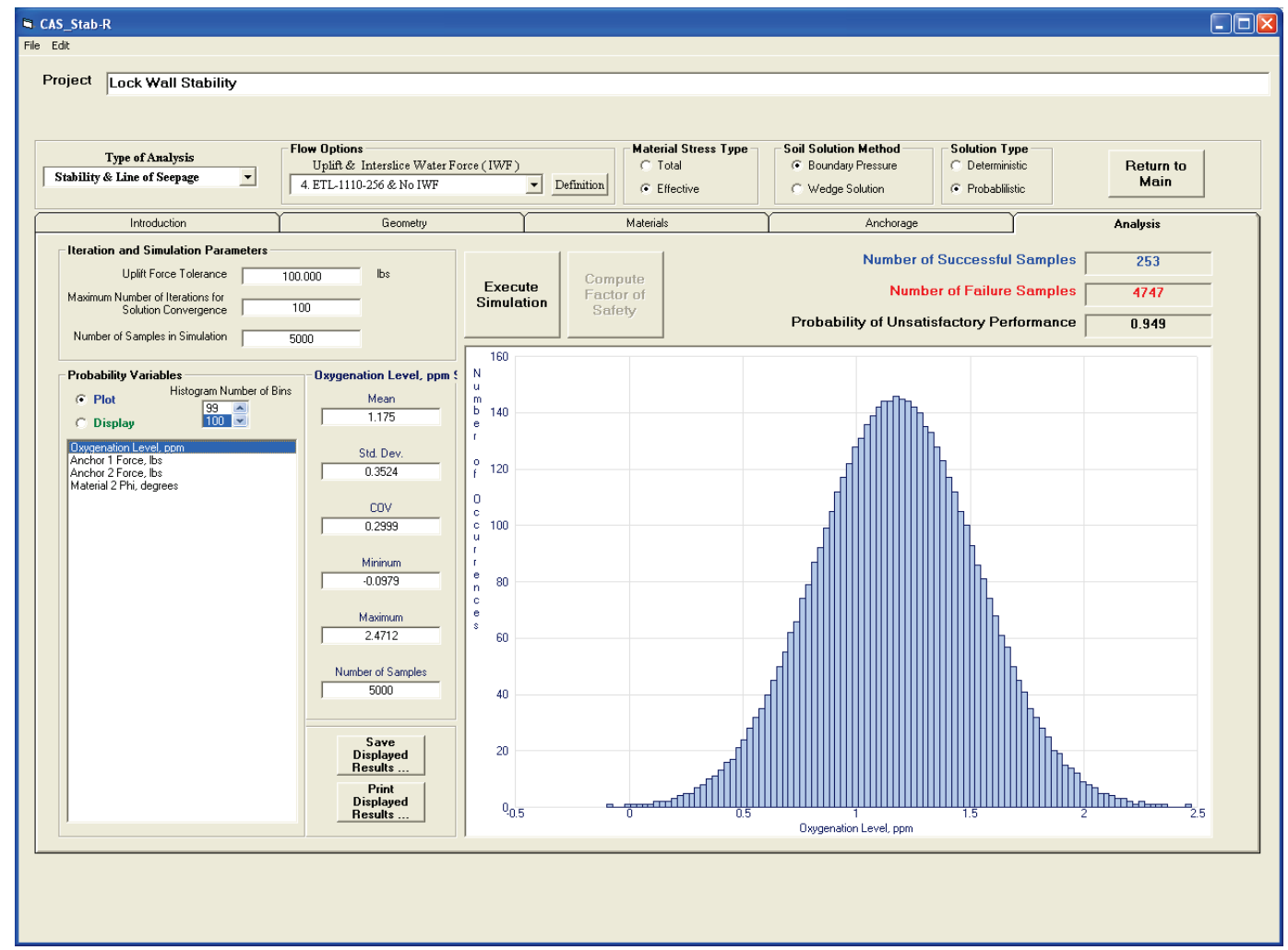


Clicking on the Anchor 1 Force variable shows the results in Figure 4-43. The histogram shows a normal distribution of values, and the statistics frame shows the distribution is centered at the mean value of $41,113 \mathrm{lb}$ with a standard deviation of 2,055 lb. These values match the user-defined distribution specified in the Probability Variable Input window because the ASM design point matched the mean values of the user-defined distribution for this variable. The Anchor 2 Force variable distribution is the same as that of the Anchor 1 Force and is not shown.

Figure 4-43. Anchor 1 lock-off force variable distribution.

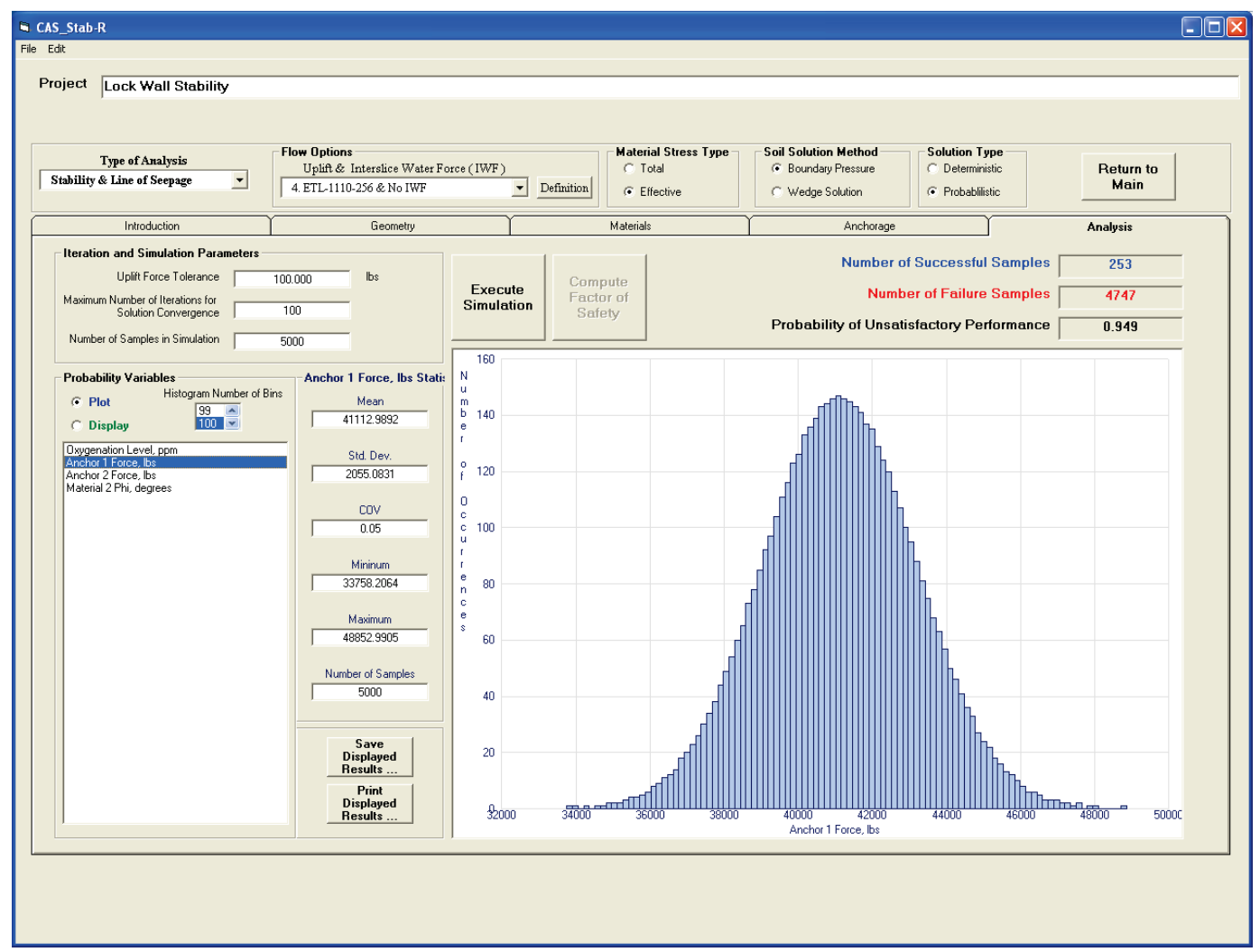

Clicking on the Material 2 Phi variable shows the results in Figure 4-44. The histogram shows a normal distribution of values, and the statistics frame shows the distribution is centered at the mean value of $27.611 \mathrm{deg}$ with a standard deviation of $3.0011 \mathrm{deg}$. Recall that the user-defined distribution for the Material 2 Phi had a mean value of 30 deg with a standard deviation of $3 \mathrm{deg}$. The ASM procedure determined the design point for importance sampling with a mean value for Phi of $27.611 \mathrm{deg}$. The standard deviation matches the user-supplied value as expected. 


\subsubsection{Effect of number of samples in the dataset on PUP}

The resulting importance sampling simulation returns the PUP value for the original user-defined distributions for the variables. This value is calculated from the ASM-centered distribution for each variable using the concept expressed in Equation 2.16 and shown in Figure 2-15.

A sufficient number of samples are required to produce a simulation that yields a reliable result. Too few samples will not provide a satisfactory distribution of values, which presents an inaccurate result. Simulations were executed with various Number of Sample settings as shown in Table 4-2. The results in the table indicate that the 5,000-sample dataset produced the identical PUP to the 10,000-sample dataset. Therefore, convergence of the PUP value occurred prior to or at 5,000 samples. The 1,000-sample dataset produced a PUP of 0.943 , which is a difference of only $0.6 \%$.

Figure 4-44. Material 2 Phi (internal friction angle) distribution.

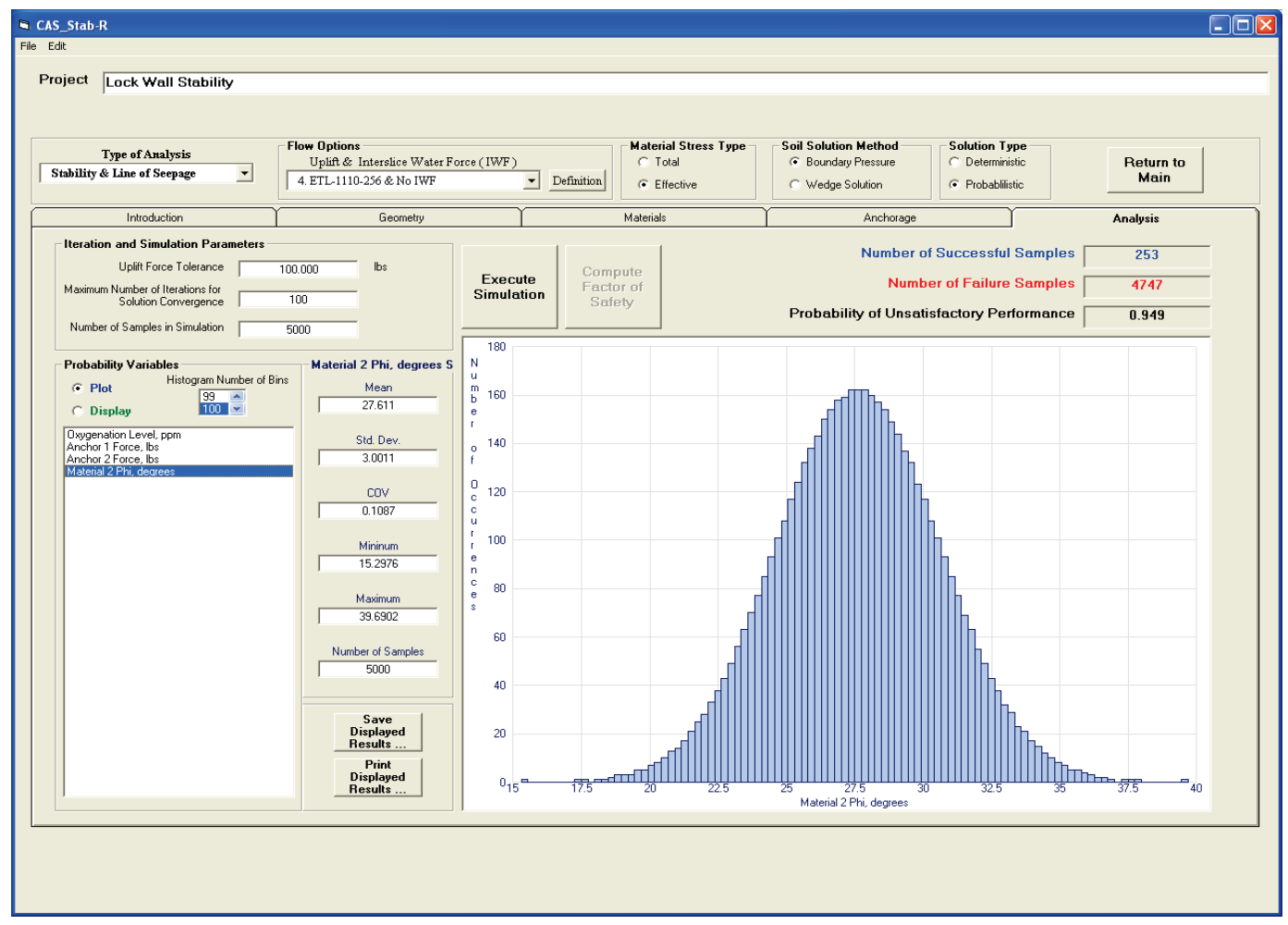


Table 4-2. PUP values for Number of Samples settings.

\begin{tabular}{|c|c|}
\hline Number of Samples & PUP \\
\hline 1,000 & 0.943 \\
\hline 5,000 & 0.949 \\
\hline 10,000 & 0.949 \\
\hline
\end{tabular}

The user must run simulations with a variety of Number of Samples settings to determine the number needed for the PUP value to converge. The authors recommend a minimum dataset size of 1,000 samples.

\subsubsection{Stability example with RAL example problem settings}

A simulation was performed for the lock wall model stability analysis example (Example 2) of section 4.3 using the anchorage and corrosion settings from the RAL example problem (Example 1) presented in section 4.2. That is, the LOS was assigned a value of 33.3 years, and the anchorages were assigned mean values of 40 kips with standard deviation values of 5 kips. The corrosion method selected was the oxygenation level with an oxygenation level mean value of $1.175 \mathrm{ppm}$ and a standard deviation value of 0.3525 for both examples as well as this analysis. The PUP that resulted for this simulation was 0.975 . 


\section{Summary and Conclusions}

This report describes a method to determine the TTF of an anchor system that is corroding and outlines the implementation of this method in the CAS_Stab-R software package. This method is based on the statistical analysis of ERDC's recently published seven-strand corroded cable pulltest results (Ebeling et al. 2016). The method estimates the time of life for user-specified lengths of service. The uncertainty of the capacity of a corroded cable and the level of corrosion that has occurred are introduced into the probabilistic estimate.

A method for a probabilistic analysis of a concrete navigation structure's resistance to sliding after a given length of time in service is also described. The factors that cause uncertainty to exist and the uncertainty implementation in the analysis are presented.

The software CAS_Stab-R was developed for inclusion into the CASE software library maintained at the ERDC by the Information and Technology Laboratory. CAS_Stab-R implements the methods for the probabilistic TTF of an anchor cable that is corroding and for the probabilistic analysis of a concrete navigation structure against sliding. CAS_Stab-R provides a Visual Modeler for input of a navigation structure model and output of the probabilistic results. Deterministic solution of the FOS against sliding for the structure model is also available. The user interface for CAS_Stab-R has been described.

For effective use of CAS_Stab-R, the user must have access to the following information:

- dimensions and material properties of the concrete structure

- topology and material properties of the foundation and retained soil materials (if present)

- mean density of the water

- foundation drain configuration (if present)

- post-tension anchorage configuration

- length of time the anchorage has been in service

- corrosion rate of anchored cables is also required. This is obtained from one of three sources: (1) a known corrosion rate of the anchor cables. (2) the level of oxygen in the moisture around the anchor cables (which correlates to a corrosion rate value), or (3) an NDT-determined 
size of the corroded anchor cable strands. Note that the rate of corrosion is obtained by assuming that there is no corrosion of the cable at installation and assuming a linear rate of corrosion until the NDT measurement is made.

CAS_Stab-R utilizes the information listed in the last two items of the preceding bulleted list for determination of the current cable capacity using linear interpolation.

Probabilistic analysis is implemented in CAS_Stab-R due to the variable nature of several of the engineering and environmental material parameters being considered. These include the following:

- lock-off loading force applied to the anchor cables at installation

- corrosion determination parameters (corrosion rate, oxygenation level, or NDT measurement)

- horizontal earth pressure coefficient for silt and soil materials

- cohesion, internal friction angle, and horizontal pressure coefficient for each rock material. These same parameters are also variables for soil regions when a wedge-based, sliding stability solution scheme is applied to the soil regions rather than a boundary pressure solution.

CAS_Stab-R allows for a correlation coefficient, $\rho$, to be specified for the cohesion and internal friction angle properties. ${ }^{1}$ These two properties are often related, and the interdependence can be reflected in the probabilistic analysis.

Example problems that serve as a tutorial for the use of CAS_Stab-R and the engineering methodologies that CAS_Stab-R software implements have been provided.CAS_Stab-R provides a tool for design engineers to examine remaining anchor lives and structural stability against sliding, incorporating ERDC's recently published corroded cable capacities as the basis for estimating the current anchorage capacities that exist at a navigation structure.

\footnotetext{
${ }^{1} \rho$ will range in value between -1.0 and 1.0.
} 


\section{References}

Ang, A. H-S., and W. H. Tang. 1984. Probability concepts in engineering planning and design. Volume II: Decision, risk, and reliability. New York: John Wiley \& Sons.

Baecher, G. B., and J. T. Christian. 2003. Reliability and statistics in geotechnical engineering. New York: John Wiley \& Sons.

Barton, N. 1973. Review of a new shear strength criterion for rock joints. Engineering Geology 7:287-332.

Barton, N., S. Bandis, and K. Bakhtar. 1985. Strength, deformation, and conductivity coupling of rock joints. International Journal of Rock Mechanics, Mining Science, and Geomechanics Abstracts 22(3):121-140.

Ditlevsen, O. 1981. Uncertainty modeling with supplications to multidimensional civil engineering systems. New York: McGraw-Hill.

Ebeling, R. M., M. T. Fong, A. Chase, Sr., and E. Arredondo. 2008. Fragility analysis of a concrete gravity dam and its system response curve computed by the analytical program GDLAD_Sloping_Base. ERDC TR-08-3. Vicksburg, MS: U.S. Army Engineer Research and Development Center.

Ebeling, R. M., M. T. Fong, J. L. Wibowo, and A. Chase, Sr. 2012. Fragility analysis of a concrete gravity dam embedded in rock and its system response curve computed by the analytical program GDLAD_Foundation. ERDC TR-12-4. Vicksburg, MS: U.S. Army Engineer Research and Development Center.

Ebeling, R. M., B. C. White, J. A. Evans, R. W. Haskins, and E. L. Miller. 2016. Corrosion induced loss of capacity of post-tensioned seven wire strand cable used in multistrand anchor systems installed at Corps projects. ERDC/ITL TR-16-4. Vicksburg, MS: U.S. Army Engineer Research and Development Center.

Ebeling, R. M., B. C. White, and M. T. Fong. 2013. Simulation and advanced second moment reliability analyses of pile groups using CPGA-R. ERDC TR-13-2. Vicksburg, MS: U.S. Army Engineer Research and Development Center.

Ebeling, R. M., M. T. Fong, A. Chase, Sr., and E. Arredondo. 2008. Fragility analysis of a concrete gravity dam and its system response curve computed by the analytical program GDLAD_Sloping_Base. ERDC TR-08-3. Vicksburg, MS: U.S. Army Engineer Research and Development Center.

Ebeling, R. M., M. T. Fong, J. L. Wibowo, and A. Chase, Sr. 2012. Fragility analysis of a concrete gravity dam and its system response curve computed by the analytical program GDLAD_Foundation. ERDC TR-12-4. Vicksburg, MS: U.S. Army Engineer Research and Development Center.

Haskins, R., J. Evans, and R. Ebeling. 2016a. Development of a new nondestructive inspection strategy for corroded multistrand anchor cables. ERDC/CHL CHETN-IX-42. Vicksburg, MS: U.S. Army Engineer Research and Development Center. 
Haskins, R., B. White, R. Ebeling, and J. Evans. 2016b. Relating corroded seven-strand, posttensioned cable cross-sectional properties to load capacity. Hindawi

Publishing Corporation, Journal of Engineering Vol. 2016, Article ID 5719156. http://dx.doi.org/10.1155/2016/5719156

Hasofer, A. M., and N. C. Lind. 1974. Exact and invariant second moment code format. Journal of the Eng. Mech. Division, ASCE 100(1):111-121.

Headquarters, U.S. Army Corps of Engineers (HQUSACE). 1981. Sliding stability for concrete structures. ETL 1110-2-256. Washington, DC: Headquarters, U.S. Army Corps of Engineers.

Murphy, W. L., R. M. Ebeling, and J. M. Andersen. 2002. Assessment of geology as it pertains to modeling uplift in jointed rock: a basis for inclusion of uncertainty in flow models. ERDC TR-02-2. Vicksburg, MS: U.S. Army Engineer Research and Development Center.

Rackwitz, R., and B. Fiessler. 1976. Note on discrete safety checking when using nonnormal stochastic models for basic variables. Loads Project Working Session, 489-494. Cambridge, MA: Massachusetts Institute of Technology.

Rackwitz, R., and B. Fiessler. 1978. Structural reliability under combined random load sequences. Computer and Structures 9(5):489-494. Oxford: Pergamon Press.

Rosenblatt, M. 1952. Remarks on a multivariate transformation. Annals of Mathematical Statistics 23(3):470-472. 


\section{Appendix A: CAS_Stab-R File Formats}

This appendix describes the format of the input files used by CAS_Stab-R to store model settings and RAL settings. Two file types are described. The first is the file type that provides the model information for the Stability Analysis function of CAS_Stab-R. These files are given the extension .cas. The second file type provides model information for the RAL function of CAS_Stab-R. These files are given the extension .ral.

\section{A.1 CAS file format}

CAS files are used to provide model information to the visual modeler for the CAS_Stab-R Stability Analysis Function. These files are ASCII format (text) files. It is possible to create a CAS file using a text editor such as Windows Notepad. Most often these files are created using the File - Save menu option in CAS_Stab-R Stability Analysis. They are loaded into the CAS_Stab-R visual modeler with the File - Open menu option.

CAS files are organized as keyword files. Each line contains a threecharacter keyword followed by parameter values for the keyword. Keywords are not case sensitive. Lines that do not begin with a valid keyword are ignored. CAS_Stab-R recognizes the following list of keywords in a CAS file. Descriptive keywords are in bold and are three characters in length. They are followed by their relevant parameters. All are separated by spaces.

Some of these keywords are optional and do not need to be specified. The mandatory codes for an analysis are nnd, nrg, gmw, mat, nod, wat, reg, inc, tol. Keywords nnd, nrg should be placed at the beginning of file. Keywords preceded by “*” denote multiple entries possible for the same code word.

\begin{tabular}{|c|c|}
\hline $\begin{array}{r}\text { nrg } \text { num_regions } \\
\text { num_regions }\end{array}$ & $\begin{array}{l}\text { Number of material regions } \\
\text { Number of regions in the model }\end{array}$ \\
\hline $\begin{array}{r}\text { nnd } \text { nnodes } \\
\text { nnodes }\end{array}$ & $\begin{array}{l}\text { Number of nodes } \\
\text { Number of nodes in the model }\end{array}$ \\
\hline $\begin{array}{l}{ }^{*} \operatorname{nod} \text { node_id, } x, y \\
\text { node_id } \\
\mathrm{x}\end{array}$ & $\begin{array}{l}x-, y \text {-coordinates of nodes defining regions } \\
\text { ID of node } \\
x \text {-coordinate of node }\end{array}$ \\
\hline
\end{tabular}


y $\quad y$-coordinate of node

gmw gamma_water Unit weight of water gamma_water Unit weight of water value (Default $=62.4$

$\mathrm{lb} / \mathrm{ft} 3)$

gmc gamma_concrete Unit weight of concrete

gamma_concrete Value of the unit weight of concrete

pol $u s p, d s p \quad$ Surface water or piezometric elevations

usp Surface water elevation on the upstream side of

dsp Surface water elevation on the downstream

side of structure

*mat material_id,gamma_moist, gamma_saturated, unit_weight, material_type, C_Phi_Correlation, K-type, MeanE, JRC

Material properties

material_id Material number

gamma_moist Moist unit weight of material

gamma_saturated Saturated unit weight of material

unit_weight Unit weight of material

material_type $\quad=1$, Rock

$=2$, Concrete

$=3$, Soil

C_Phi_Correlation Correlation between the material's Cohesion and Internal Friction Angle properties

K-type Hydraulic Aperture Type (unused in

CAS_Stab-R)

MeanE Mean size of hydraulic aperture (unused in

CAS_Stab-R)

JRC Hydraulic aperture Joint Roughness

Coefficient (unused in CAS_Stab-R)

*med mat_id, dist_type,paramA,paramB,paramC, paramD

material cohesion probability distribution

mat_id

Material ID

dist_type

Type of probability distribution

$=0$, Normal

$=1$, Bounded Normal 


$\begin{array}{ll} & =2, \text { Lognormal } \\ & =3, \text { Bounded Lognormal } \\ & =4, \text { Uniform } \\ & =5, \text { Triangular } \\ & \text { Mean value for dist_type }=0-3 \\ \text { paramA } & \text { Start value for dist_type }=4 \text { or } 5 \\ & \text { Standard deviation value for dist_type }=0-3 \\ \text { paramB } & \text { End value for dist_type }=4 \\ & \text { Mid-point value for dist_type }=5 \\ \text { paramC } & \text { Start value for dist_type }=1,3 \\ & \text { End value for dist_type }=5 \\ \text { paramD } & \text { Don't care for dist_type }=0,2,4 \\ & \text { End value for dist_type }=1,3 \\ & \text { Don't care for dist_type }=0,2,4,5\end{array}$

*mpd mat_id, dist_type, paramA, paramB, paramC, paramD

material internal friction angle, phi, probability distribution

Parameters are as for keyword med as above.

* $\mathbf{m k d}$ mat_id, dist_type,paramA, paramB, paramC, paramD material horizontal earth pressure coefficient probability distribution

Parameters are as for keyword med as above.

*reg region_id, water_type,water_id, material_id Area of homogeneous material

region_id Global region number

water_type (Unused in CAS_Stab-R)

water_id (Unused in CAS_Stab-R)

material_id Material number associated with this region

*rgn region_id, $n \_n o d e s, n \_i d\left(1: n \_n o d e s\right)$ nodes defining the region region_id

Global region number

$\mathrm{n} \_$nodes

The number of nodes defining this region

$\mathrm{n} \_\mathrm{id}\left(1: n \_\right.$nodes $)$

All nodes defining this region. The coordinates

for the node IDs can be found in the nod section. These nodes are listed in the counterclockwise direction ( 1 to $n \_$nodes) from the $x$-coordinate nearest to the I-Wall. 


\begin{tabular}{|c|c|}
\hline $\begin{array}{l}\text { gap node_id_top, } \\
\text { node_id_bottom }\end{array}$ & Gap location \\
\hline node_id_top & Node ID of node at top of fracture \\
\hline node_id_bottom & Node ID of node at bottom of fracture \\
\hline itr max_iter & $\begin{array}{l}\text { Maximum number of iterations executed for } \\
\text { FOS analysis }\end{array}$ \\
\hline max_iter & $($ default $=100)$ \\
\hline
\end{tabular}

gal style, width, height, radius, node_id Gallery size and position style $=1$, Rectangular

width $=2$, Dome Ceiling height radius Base width node_id Wall height If style $=2$, radius of the dome section Node ID assigned to bottom center of the gallery

$\mathbf{s t r} n \_n o d e s, n \_i d\left(1: n \_n o d e s\right)$ nodes defining the structure (dam, lock wall) n_nodes The number of nodes defining the structure n_id(1:n_nodes) Node IDs defining the structure. The coordinates for the node_ids can be found in the nod section. These nodes are listed in the counter-clockwise direction (1 to $n \_$nodes) beginning with the lower left corner.

stm structure_material Structure material structure_material Index of material with the structure properties

slp $n \_n o d e s, n \_i d\left(1: n \_n o d e s\right) \quad$ Nodes defining the slip line $n \_$nodes The number of nodes defining the slip line n_id(1:n_nodes) node IDs defining the slip line. The coordinates for the node_ids can be found in the nod section. These nodes are listed from left to right. 
drn drain_type, efficiency, node_id_top, node_id_bottom drain parameters drain_type Drain type

$=0$, no gallery drain

$=1$, closed gallery drain

$=2$, open gallery drain

efficiency The efficiency of the drains, in percent node_id_top The node ID of the top of the drain node_id_bottom Then node ID of the bottom of the drain

*anc analysis_type, anchor_id, lockoff_force_distribution_type, force_param A, force_paramB, force_paramC, force_paramD, node_id_top, node_id_bottom, cables_per_group,group_spacing, king_diameter, outer_diameter, pristine_capacity, strands_per_cable Anchorage parameters analysis_type

Type of anchorage analysis

$=0$, no anchor

$=1$, generate anchorage uncertainties (unused

in

$$
\text { CAS_Stab-R) }
$$

$=2$, deterministic analysis

anchor_id The ID for this anchorage lockoff_force_distribution_type type of probability distribution for the lockoff force

force_paramA

Distribution parameter A

force_paramB Distribution parameter B

force_paramC Distribution parameter C force_paramD Distribution parameter D

node_id_top The node ID of the top of the anchor node_id_bottom The node id of the bottom of the anchor cables_per_group Number of cables in each anchor bundle group_spacing Distance between each cable bundle king_diameter Diameter of the cable king wire outer_diameter Diameter of the cable outer wire pristine_capacity Anchor cable strength in pristine condition strands_per_cable Number of wire strands in each cable The king_diameter, outer_diameter, pristine_capacity and strands_per_cable are set to default values of 0.2 in., 0.198 in., 
$61688.68 \mathrm{lb}$ and 7, respectively. They are unavailable for user modification in CAS_Stab-R.

tol tolerance tolerance
Horizontal imbalance force tolerance Tolerance for convergence of horizontal imbalance force for Factor of Safety iterative solution

slt silt_height,gamma_moist,gamma_saturated silt parameters silt_height Height at the top of the silt layer gamma_moist The density of moistened silt gamma_saturated The density of saturated silt

sld dist_type, paramA, paramB, paramC, paramD silt lateral earth pressure coefficient distribution parameters
dist_type
Type of probability distribution
$=0$, Normal
$=1$, Bounded Normal
$=2$, Lognormal
$=3$, Bounded Lognormal
$=4$, Uniform
$=5$, Triangular
paramA
Mean value for dist_type $=0-3$
Start value for dist_type $=4$ or 5
paramB Standard deviation value for dist_type $=0-3$
End value for dist_type $=4$
Mid-point value for dist_type $=5$
paramC Start value for dist_type $=1,3$
End value for dist_type $=5$
Don't care for dist_type $=0,2,4$
paramD
End value for dist_type $=1,3$

*sol soil_id, soil_height,gamma_moist,gamma_saturated layer parameters

soil_id

soil_height

gamma_moist

gamma_saturated
ID number for this soil layer

Height at the top of the soil layer

The density of moistened soil

The density of saturated soil 
*sld soil_id, dist_type,paramA, paramB,paramC, paramD soil lateral earth pressure coefficient distribution parameters soil_id ID number for this soil layer dist_type

Type of probability distribution

$=0$, Normal

$=1$, Bounded Normal

$=2$, Lognormal

$=3$, Bounded Lognormal

$=4$, Uniform

$=5$, Triangular

paramA

Mean value for dist_type $=0-3$

Start value for dist_type $=4$ or 5

paramB

Standard deviation value for dist_type $=0-3$

End value for dist_type $=4$

Mid-point value for dist_type $=5$

paramC

Start value for dist_type $=1,3$

End value for dist_type $=5$

Don't care for dist_type $=0,2,4$

paramD

End value for dist_type $=1,3$

flw flow_option

Flow condition specification

flow_option

$=4$, Joint flow and no IWF

$=5$, Joint flow and near hydrostatic

$=6$, Joint flow and joint flow

$\tan$ type_of_analysis Analysis Type

type_of_analysis $\quad=1$, probabilistic

$=2$, deterministic

stt stress_computation_method Analysis Type stress_computation_method $=1$, total

$=2$, effective

somsoil_model_type soil_model_type pressures
Soil Model in Analysis

$=0$, Hydrostatic structural boundary

$=1$, Soil is a slip plane wedge

sms number_of_samples, pup Number of samples for the probabilistic simulation 
number_of_samples Number of samples for the simulation

pup

Probability of Unsatisfactory

Performance from a previous simulation

cor corrosion_rate_method, reduced_capacity_curve, LOS_Years, LOS_Months corroded cable capacity parameters corrosion_rate_method Method to determine the current corroded cable size

$=1$, User-specified Corrosion Rate =2, User-specified Oxygenation Level $=3$, NDT Measured Cable Size

reduced_capacity_curve Curve to compute the corroded cable capacity

diameter curve
LOS_Years
Years since anchor was put into service
LOS_Months
Years since anchor was put into service

crd dist_type, paramA, paramB, paramC, paramD user-specified corrosion rate distribution

$\begin{array}{ll}\text { dist_type } & \text { Type of probability distribution } \\ & =0, \text { Normal } \\ & =1, \text { Bounded Normal } \\ & =2, \text { Lognormal } \\ & =3 \text {, Bounded Lognormal } \\ & =4, \text { Uniform } \\ & =5, \text { Triangular } \\ & \text { Mean value for dist_type }=0-3 \\ \text { paramA } & \text { Start value for dist_type }=4 \text { or } 5 \\ & \text { Standard deviation value for dist_type }=0-3 \\ \text { paramB } & \text { End value for dist_type }=4 \\ & \text { Mid-point value for dist_type }=5 \\ \text { paramC } & \text { Start value for dist_type }=1,3 \\ & \text { End value for dist_type }=5 \\ \text { paramD } & \text { Don't care for dist_type }=0,2,4 \\ & \text { End value for dist_type }=1,3\end{array}$

old dist_type, paramA, paramB, paramC, paramD user-specified oxygenation level distribution 


$\begin{array}{ll}\text { dist_type } & \text { Type of probability distribution } \\ & =0, \text { Normal } \\ & =1, \text { Bounded Normal } \\ & =2, \text { Lognormal } \\ & =3 \text {, Bounded Lognormal } \\ & =4, \text { Uniform } \\ & =5, \text { Triangular } \\ & \text { Mean value for dist_type }=0-3 \\ \text { paramA } & \text { Start value for dist_type }=4 \text { or } 5 \\ & \text { Standard deviation value for dist_type }=0-3 \\ \text { paramB } & \text { End value for dist_type }=4 \\ & \text { Mid-point value for dist_type }=5 \\ \text { paramC } & \text { Start value for dist_type }=1,3 \\ & \text { End value for dist_type }=5 \\ \text { paramD } & \text { Don't care for dist_type }=0,2,4 \\ & \text { End value for dist_type }=1,3\end{array}$

ndd dist_type, paramA, paramB, paramC, paramD NDT measured cable size distribution dist_type

Type of probability distribution

$=0$, Normal

$=1$, Bounded Normal

$=2$, Lognormal

$=3$, Bounded Lognormal

$=4$, Uniform

$=5$, Triangular

paramA

Mean value for dist_type $=0-3$

Start value for dist_type $=4$ or 5

paramB

Standard deviation value for dist_type $=0-3$

End value for dist_type $=4$

Mid-point value for dist_type $=5$

paramC

Start value for dist_type $=1,3$

End value for dist_type $=5$

Don't care for dist_type $=0,2,4$

paramD $\quad$ End value for dist_type $=1,3$

uni units

units
System of units for measurements

$=1$, English units

$=2$, SI units 


$\begin{array}{ll}\text { prj } \text { project_name } & \text { Project Name } \\ \text { project_name } & \begin{array}{l}\text { Project Name for the model } \\ \text { configuration }\end{array}\end{array}$

\section{A.2 RAL file format}

RAL files are used to provide information for the CAS_Stab-R RAL Analysis Function. These files are ASCII format (text) files. It is possible to create an RAL file using a text editor such as Windows Notepad. Most often these files are created using the File - Save menu option in CAS_Stab-R RAL window. They are loaded into the CAS_Stab-R visual modeler with the File - Open menu option.

RAL files are free format in style. However, each input parameter must appear in the file in the order specified. The order of inputs is described below. Text in the color green are lines for an example file.

The first line contains the project name enclosed in quotation. "Project 1"

The next line contains the length of service for the anchorage in years and months.

0,0

The next line contains the number of anchors with lock-off load distribution information in this file.

1

The next line contains the currently selected anchor when this file was saved/created.

1

For each anchor in the file there are now four lines, which contain the following:

- Anchor ID number

- King wire diameter, outer wire diameter, pristine cable capacity, number of wires in the cable

- Number of anchor cables per group, group spacing for the anchors

- Fourth line will be one of the following depending on the distribution type. The first parameter on the line is the distribution type index. 
- 1, mean, standard deviation, minimum, maximum (for a bounded normal distribution)

- 3, mean, standard deviation, minimum, maximum (for a bounded lognormal distribution)

- 4, minimum, maximum (for a uniform distribution)

○ 5 , minimum, mid-point, maximum (for a triangular distribution)

1

$.2, .198,61688.68,7$

12,25

$1,40000,5000,20000,60000$

The next nine lines provide distribution information for the most recently selected anchor lock-off load variable information. The nine lines contain the following information:

- Flag to indicate if this was the selected variable for distribution parameter edits

- Variable ID number

- Variable Name

- Distribution type index (options as discussed in the med keyword above)

- Distribution parameter A (as discussed in the med keyword above)

- Distribution parameter B (as discussed in the mcd keyword above)

- Distribution parameter C (as discussed in the med keyword above)

- Distribution parameter D (as discussed in the med keyword above)

- Coefficient of variation

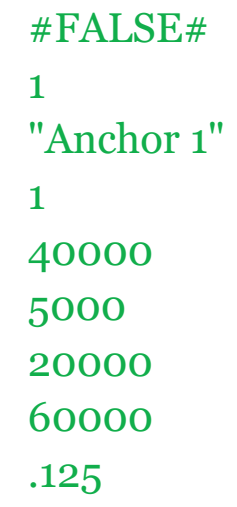


The next nine lines provide distribution information for the user-specified corrosion rate variable information. The nine lines contain the following information:

- Flag to indicate if this was the selected variable for distribution parameter edits

- Variable ID number

- Variable Name

- Distribution type index (options as discussed in the med keyword above)

- Distribution parameter A (as discussed in the med keyword above)

- Distribution parameter B (as discussed in the mcd keyword above)

- Distribution parameter C (as discussed in the mcd keyword above)

- Distribution parameter D (as discussed in the med keyword above)

- Coefficient of variation

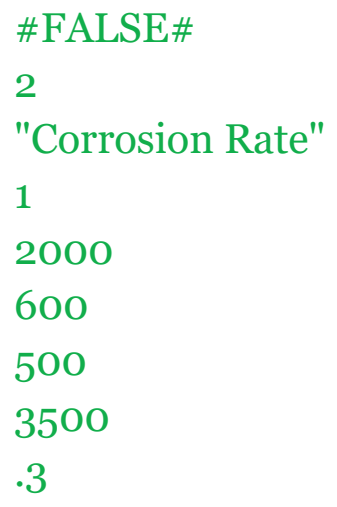

The next nine lines provide distribution information for the user-specified oxygenation level variable information. The nine lines contain the following information:

- Flag to indicate if this was the selected variable for distribution parameter edits

- Variable ID number

- Variable Name

- Distribution type index (options as discussed in the med keyword above)

- Distribution parameter A (as discussed in the med keyword above)

- Distribution parameter B (as discussed in the mcd keyword above)

- Distribution parameter C (as discussed in the mcd keyword above)

- Distribution parameter D (as discussed in the med keyword above) 
- Coefficient of variation

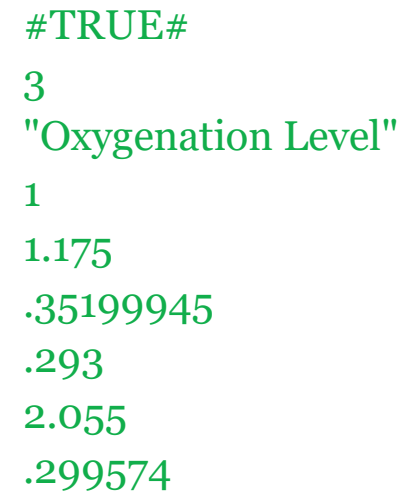

The next nine lines provide distribution information for the NDTmeasured cable area variable information. The nine lines contain the following information:

- Flag to indicate if this was the selected variable for distribution parameter edits

- Variable ID number

- Variable Name

- Distribution type index (options as discussed in the med keyword above)

- Distribution parameter A (as discussed in the med keyword above)

- Distribution parameter B (as discussed in the mcd keyword above)

- Distribution parameter C (as discussed in the med keyword above)

- Distribution parameter D (as discussed in the med keyword above)

- Coefficient of variation

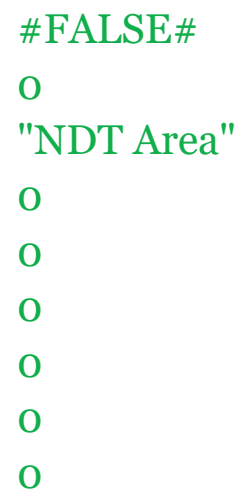

The next three lines are flags to indicate the method of determining the corroded cable size is to be used. They are in this order: 
- User-specified corrosion rate

- User-specified oxygenation level

- NDT-measured area

$$
\begin{aligned}
& \text { \#FALSE\# } \\
& \text { \#TRUE\# } \\
& \text { \#FALSE\# }
\end{aligned}
$$

The next two lines are flags to indicate the capacity curve selected for computing the reduced capacity of the corroded cable. They occur in this order:

- Short axis diameter curve

- Area curve

$$
\begin{aligned}
& \text { \#FALSE\# } \\
& \text { \#TRUE\# }
\end{aligned}
$$

The next line is the system of units for measurements. The value 1 indicates the use of English units. The value 2 indicates the use of SI units. 1

The next line is the number of bins minus 1 for histogram plots. 99

The next line is the number of points in the simulation data set. 10000

The remaining lines are the simulation data points for the lock-off load value, corrosion value, and capacity dispersion variable. For this example, 10,000 lines will follow. Only the first 10 are shown here.

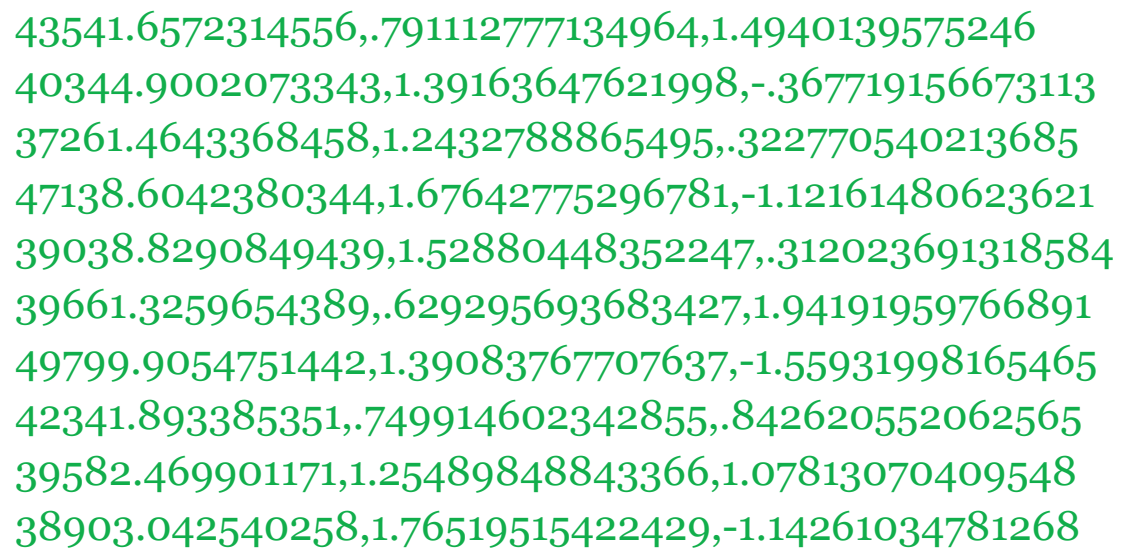




\section{Appendix B: CAS_Stab-R Deterministic Solution Examples}

This appendix describes two example concrete dam structure configurations and the use of CAS_Stab-R for the determination of the Factor of Safety (FOS) against sliding using the Deterministic Solution option. The basis for both geometrical dam/foundation configurations is the five wedge system given as an example in ETL 1110-2-256 (HQUSACE 1981). The first example is the ETL 1110-2-256 (HQUSACE 1981) problem as described in the document. The second example is the same configuration with a gallery, drain system and anchorage added.

\section{B.1 Example 1 - ETL 1110-2-256 (HQUSACE 1981) configuration}

CAS_Stab-R was used to determine the FOS for the concrete dam configuration that was given in ETL 1110-2-256 (HQUSACE 1981) for the five-wedge example. The geometry of the dam and foundation cross section is shown in Figure B-1. The potential slip plane is depicted in red in this figure.

Figure B-1. ETL 1110-2-256 (HQUSACE 1981) example geometry.

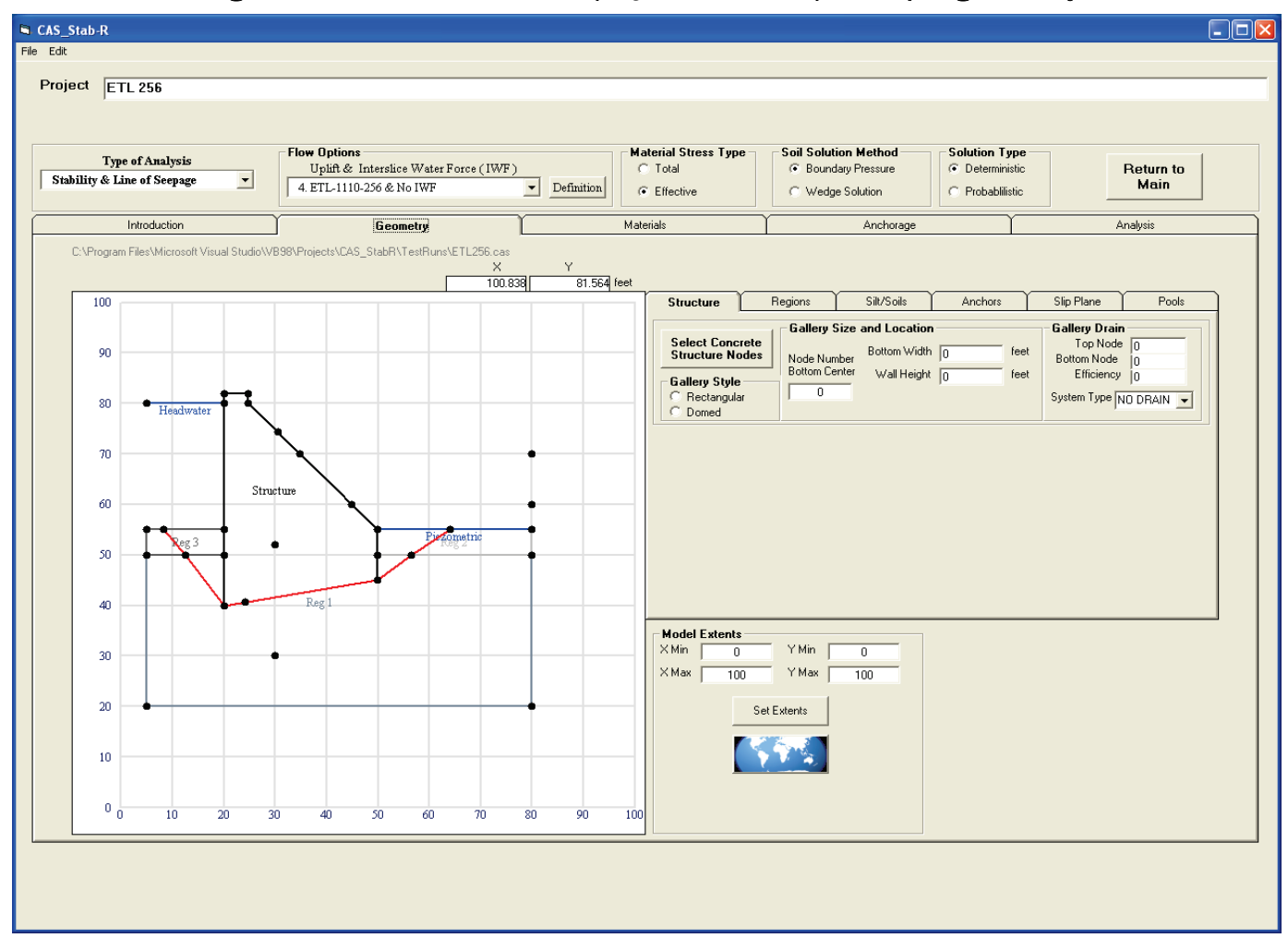


The nodes that are shown in Figure B-1 and used to define the components of the model have coordinate values listed in Table B-1. The structural wedge that contains the dam is defined by polygonal area outlined by nodes $10,11,4,13,3,2,1$, and returning to 10 in the specified counterclockwise order. The nodes that define rock region one are 18, 19, 17, 16, $11,10,15,14$, and returning to 18 . Rock region two is defined by nodes 16 , 17,8 , and 4, respectively. Rock region three is defined by nodes 14, 15, 1 , and 5, respectively. The potential slip plane line (in red in Figure B-1) is defined by nodes $6,9,10,11,12$, and 7 , in that order. The headwater pool elevation is $80 \mathrm{ft}$. The piezometric tailwater elevation is $55 \mathrm{ft}$. The density of the water is set at $62.5 \mathrm{lb} / \mathrm{ft}^{3}$.

Table B-1. Node coordinates for the ETL 1110-2-256 (HQUSACE 1981) example.

\begin{tabular}{|c|c|c|}
\hline Node & $X$-Coordinate & $Y$-Coordinate \\
\hline 1 & 20 & 55 \\
\hline 2 & 20 & 82 \\
\hline 3 & 24.76065 & 82 \\
\hline 4 & 49.87882 & 55 \\
\hline 5 & 5 & 55 \\
\hline 6 & 8.306225 & 55 \\
\hline 7 & 64.04634 & 55 \\
\hline 8 & 80 & 55 \\
\hline 9 & 12.47842 & 50 \\
\hline 10 & 20 & 40 \\
\hline 11 & 49.87882 & 45 \\
\hline 12 & 56.52636 & 50 \\
\hline 13 & 24.76065 & 80 \\
\hline 14 & 5 & 50 \\
\hline 15 & 20 & 50 \\
\hline 16 & 49.87882 & 50 \\
\hline 17 & 80 & 50 \\
\hline 18 & 5 & 20 \\
\hline 19 & 80 & 20 \\
\hline 20 & 30 & 52 \\
\hline 21 & 30 & 30 \\
\hline 22 & 30.43 & 74.35 \\
\hline
\end{tabular}




\begin{tabular}{|c|c|c|}
\hline Node & $X$-Coordinate & $Y$-Coordinate \\
\hline 23 & 24.03326 & 40.67494 \\
\hline 24 & 34.80792 & 70 \\
\hline 25 & 80 & 70 \\
\hline 26 & 5 & 80 \\
\hline 27 & 20 & 80 \\
\hline 28 & 44.85519 & 60 \\
\hline 29 & 80 & 60 \\
\hline
\end{tabular}

Four materials are defined with properties as shown in Figure B-2. The structure is assigned the properties of material 4. Regions 1, 2, and 3 are assigned the properties of materials numbered 1, 3, and 2, respectively, as shown in the Region-Material Map of Figure B-3.

Figure B-2. Material properties of the four materials utilized in the ETL 1110-2-256 (HQUSACE 1981) example.
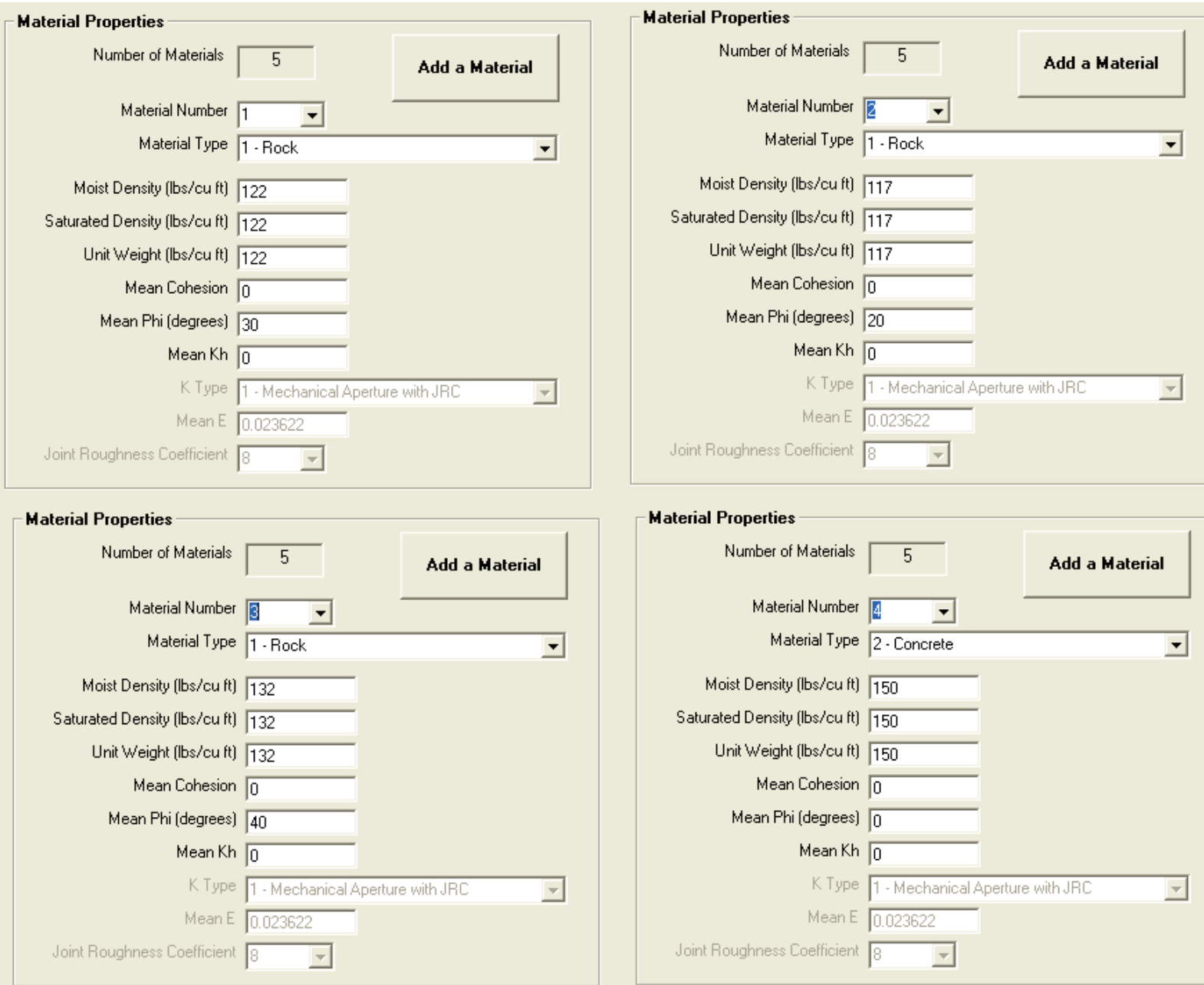
Figure B-3. Region - Material

Mapping for the ETL 1110-2-256

(HQUSACE 1981) example.

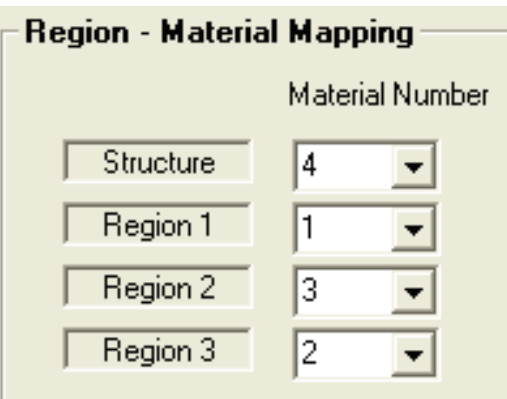

During the FOS computations, the material property value for the internal friction angle, $\phi$, applied in Equation 2-8 for a wedge is ordinarily assigned the value given to the material for the region immediately above the slip plane line. In this example, the potential slip plane lies on the interface between the structure and region 3 as seen in Figure B-1. For a geometry such as this, CAS_Stab-R utilizes the value of $\phi$ for the region immediately below the slip plane in the Equation 2-8 equilibrium force computation. The value of $\phi$ will be that of material 2 (i.e., $30 \mathrm{deg}$ ) in this example.

The result of the FOS computation for this example is 1.988 as reported in the CAS_Stab-R Analysis Tab (Figure B-4). This value is slightly lower than the value of 2.0 obtained in the ETL 1110-2-256 (HQUSACE 1981) document. This small difference is due to utilizing a smaller tolerance value of $100 \mathrm{lb}$ for horizontal equilibrium in the determination of convergence in the CAS_Stab-R solution as compared to the $18 \mathrm{olb}$ tolerance used in the ETL 1110-2-256 (HQUSACE 1981) hand computations. 
Figure B-4. ETL 1110-2-256 (HQUSACE 1981) example FOS result.

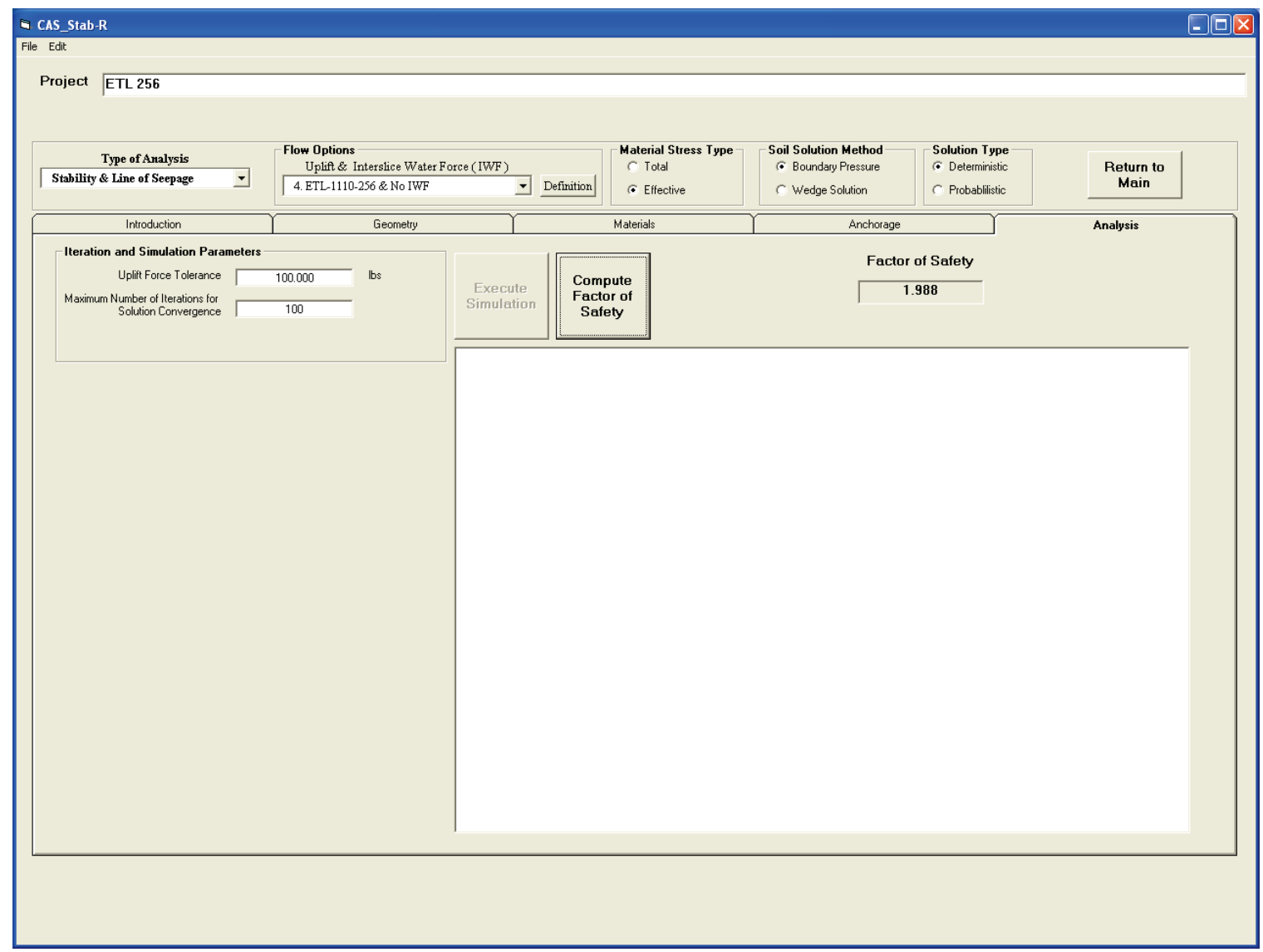

\section{B.2 Example 2 - ETL 1110-2-256 (HQUSACE 1981) configuration with anchorage, gallery, and drainage system}

For this example, the ETL 1110-2-256 (HQUSACE 1981) example discussed in the previous section was modified to include a gallery with a drainage system and an anchorage system. The model with these additions is shown in Figure B-5. The line of foundation drains is depicted in blue in this figure. Note that these drains extend below the potential slip plane (in red). The row of post-tensioned anchorage is depicted in green in this figure. Note that these multi-strand anchors extend below the potential slip plane (in red) into the foundation material. 
Figure B-5. Modified ETL 1110-2-256 (HQUSACE 1981) example geometry with added gallery, drainage, and anchorage.

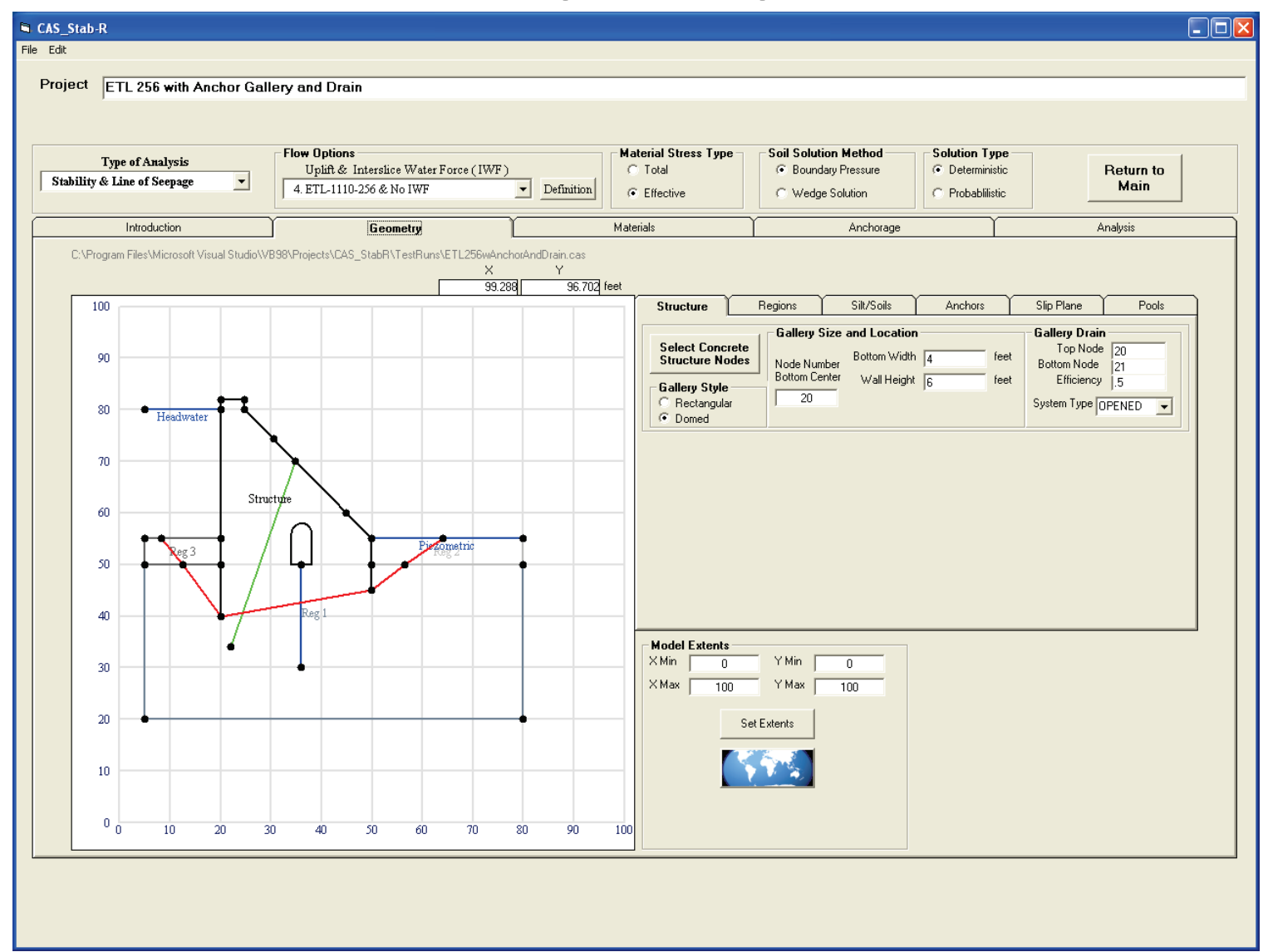

The gallery dimensions are width of $4 \mathrm{ft}$, height of $6 \mathrm{ft}$, and a domed ceiling. In CAS_Stab-R, the domed ceiling is as a semi-circle given a radius equal to one-half of the width, or $2 \mathrm{ft}$ in this example. The drainage system is chosen as an open system that extends from node 20 at the top to node 21 at the bottom with an efficiency of 0.5. Further details on the program options for open or closed drainage systems are described in Ebeling et al. (2012). CAS_Stab-R uses the same drainage models as GDLAD_Foundation software (Ebeling et al. 2012).

The anchorage consists of 12 cable groupings with each grouping spaced at $25 \mathrm{ft}$ intervals along the axis of the dam. The lock-off force applied to each cable is 20,000 lb. These settings are shown in Figure B-6. The top of the anchorage is located at node 23 , and the bottom is located at node 27 as shown in Figure B-7. 
Figure B-6. Modified ETL 1110-2-256 (HQUSACE 1981) anchorage settings.

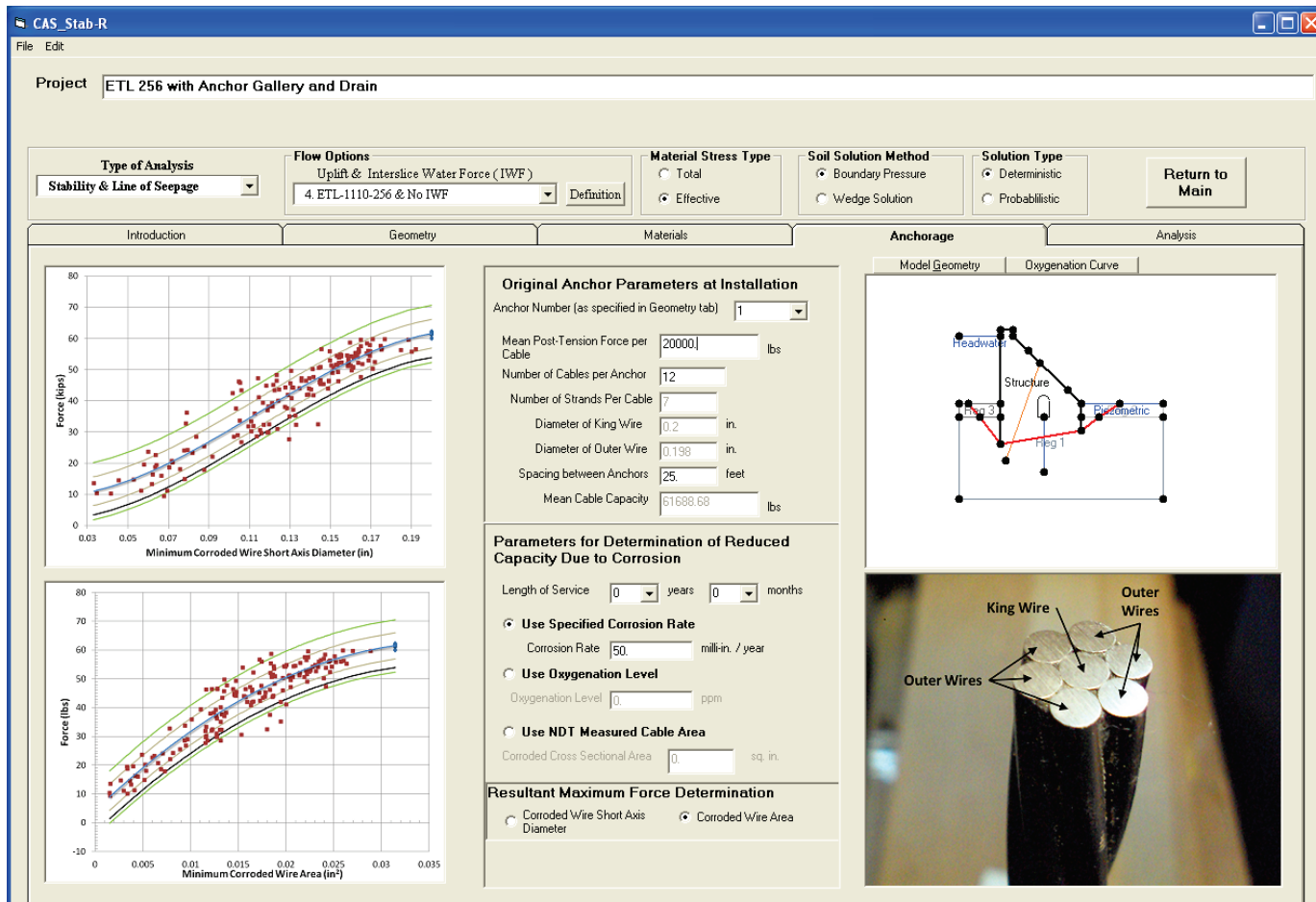

Figure B-7. Modified ETL 1110-2-256 (HQUSACE 1981) anchor location.

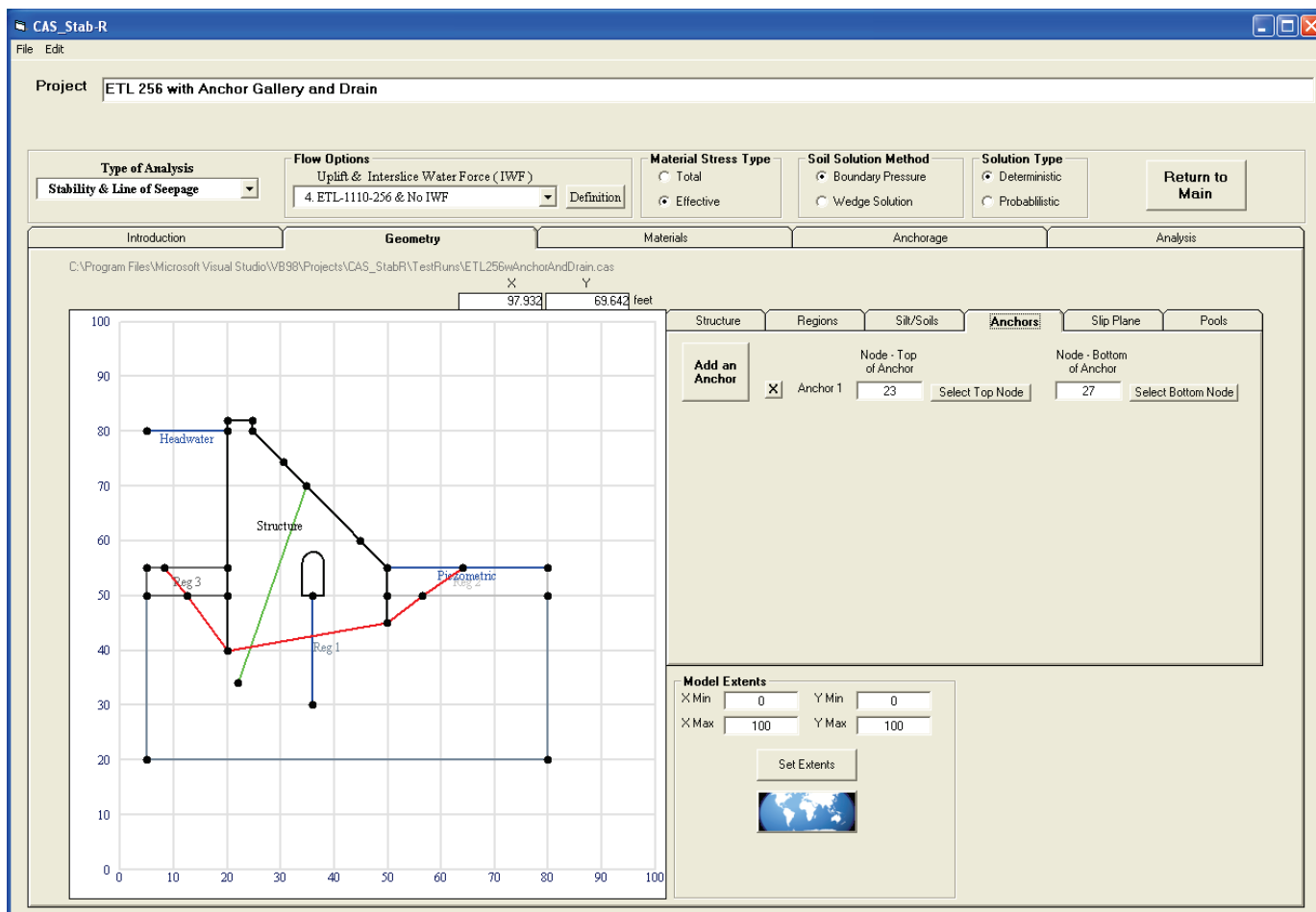


The deterministic analysis for the second example produced an FOS of 2.520 as reported in the CAS_Stab-R Analysis Tab (Figure B-8). The result of adding the drainage system and the multi-strand anchorage resulted in an increase of the computed FOS from 1.988 (Example 1) to 2.520 (Example 2).

Figure B-8. Modified ETL 1110-2-256 (HQUSACE 1981) FOS result.

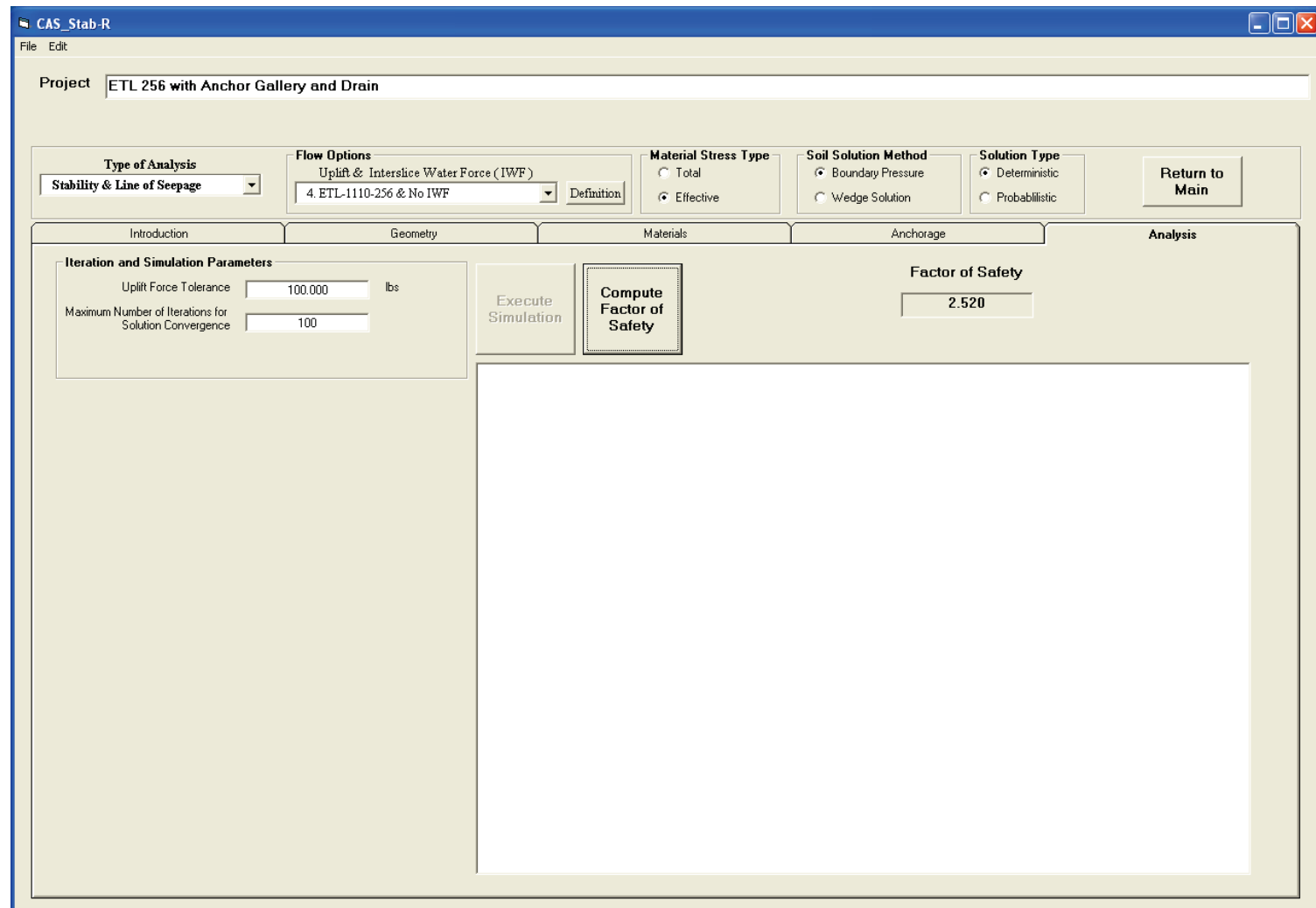




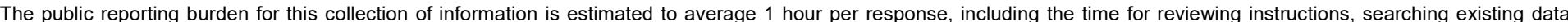

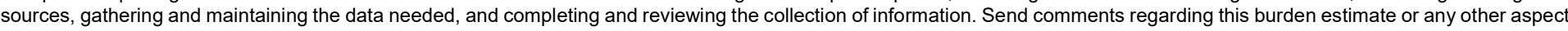

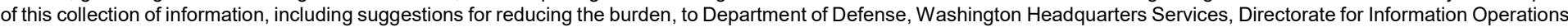

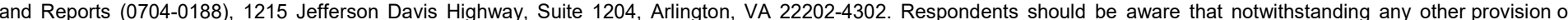
law, no person shall be subject to any penalty for failing to comply with a collection of information if it does not display a currently valid OMB control number.

PLEASE DO NOT RETURN YOUR FORM TO THE ABOVE ADDRESS.

\begin{tabular}{l|l|l}
\hline $\begin{array}{l}\text { 1. REPORT DATE } \\
\text { December } 2017\end{array}$ & $\begin{array}{l}\text { 2. REPORT TYPE } \\
\text { Final Report }\end{array}$ & 3. DATES COVERED (FrOm - To) \\
\hline
\end{tabular}

\section{TITLE AND SUBTITLE}

Corroded Anchor $\underline{\text { Structure Stability/ }}$

Reliability (CAS_Stab-R) Software for Hydraulic Structures

5a. CONTRACT NUMBER

5b. GRANT NUMBER

5c. PROGRAM ELEMENT NUMBER

\section{AUTHOR(S)}

Terry W. Warren, Barry C. White, and Robert M. Ebeling

5d. PROJECT NUMBER

5e. TASK NUMBER

5f. WORK UNIT NUMBER

33143

7. PERFORMING ORGANIZATION NAME(S) AND ADDRESS(ES)

Information Technology Laboratory

U.S. Army Engineer Research and Development Center

3909 Halls Ferry Road

Vicksburg, MS 39180-6199

9. SPONSORING/MONITORING AGENCY NAME(S) AND ADDRESS(ES)

U.S. Army Corps of Engineers

Washington, DC 20314-1000

8. PERFORMING ORGANIZATION REPORT NUMBER

ERDC/ITL TR-17-3

10. SPONSOR/MONITOR'S ACRONYM(S)

HQUSACE

11. SPONSOR/MONITOR'S REPORT NUMBER(S)

\section{DISTRIBUTION/AVAILABILITY STATEMENT}

Approved for public release; distribution is unlimited.

\section{SUPPLEMENTARY NOTES}

\section{ABSTRACT}

This report describes software that provides a probabilistic estimate of time-to-failure for a corroding anchor strand system. These anchor systems are installed to preserve and extend the service life of U.S. Army Corps of Engineers hydraulic structures.

Corrosion reduces the cross-section area of steel cables until the cable capacity is less than the tension force applied when the anchor cable was initially installed. When enough material is lost from the cable anchor that the cable capacity is less than the lock-off load of the anchor, the anchor will fail and no longer provide stability to the structure. A series of unique pull-test experiments conducted by Ebeling et al. (2016) at the U.S. Army Engineer Research and Development Center provided the required statistical relationships of reduced sevenstrand cable capacity to (1) corroded cross-section area and to (2) corroded cross-section minimum short axis diameter for failed cable strands.

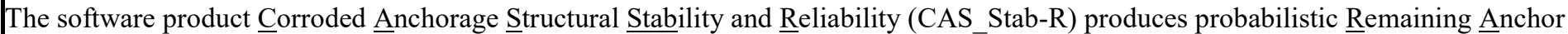
Life time estimates for anchor cables based upon the direct corrosion rate for the installation. CAS_Stab-R can also perform a probabilistic analysis to determine the Probability of Unsatisfactory Performance for a structural model cross section founded on rock.

\section{SUBJECT TERMS}

Anchorage (Structural engineering), Computer software, Hydraulic structures-Maintenance and repair, Service life (engineering), Steel-Corrosion, Structural stability

16. SECURITY CLASSIFICATION OF:

\begin{tabular}{|l|c|c|l|} 
a. REPORT & b. ABSTRACT & c. THIS PAGE & OF ABSTRACT \\
Unclassified & Unclassified & Unclassified & SAR
\end{tabular}

18. NUMBER 19a. NAME OF RESPONSIBLE PERSON

OF PAGES Robert M. Ebeling

196 19b. TELEPHONE NUMBER (Include area code) 601-634-3458 